Christian Hockenjos

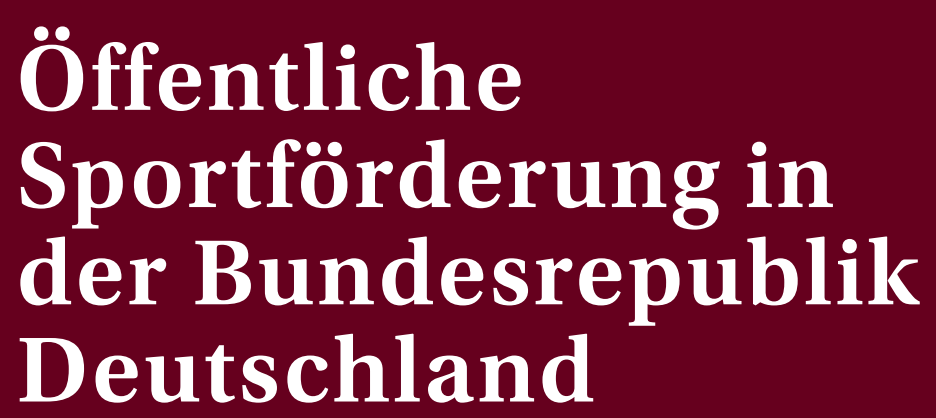

Darstellung und finanztheoretische Analyse 


\section{Öffentliche Sportförderung in der Bundesrepublik Deutschland}

Die Dissertation stellt die bundesdeutsche Sportförderpraxis in quantitativer wie qualitativer Hinsicht über einen Zeitraum von 25 Jahren dar, wobei - im Gegensatz zu bisherigen Veröffentlichungen - die kommunale Sportförderung im Mittelpunkt der Betrachtung steht. Einen zweiten Schwerpunkt bildet die theoretische Analyse des Status quo. Wieso verausgabt der Staat in einem marktwirtschaftlichen System Milliarden für den Sport, warum fließen die öffentlichen Gelder fast ausschließlich in Richtung des organisierten Vereinssports, und wie erklärt sich die Höhe von Sportbudgets oder die Tatsache, daß Sportpolitik in erster Linie eine kommunale Angelegenheit ist?

Die Daten für die zahlreichen empirischen Analysen wurden größtenteils vom Autor selbst erhoben. So fließen u.a. Abfragen kommunaler Sportförderrichtlinien und Sportbudgets sowie eine Studie zum Werbewert von Sportstätten in die Arbeit mit ein.

Christian Hockenjos wurde 1963 in Lahr geboren. Das Studium der Volkswirtschaftslehre an der Albert-Ludwigs-Universität Freiburg schloß er 1991 als Diplom-Volkswirt ab. Seitdem ist er als Dozent für Betriebswirtschaftslehre und als freier Journalist tätig. 1995 promovierte er bei Prof. Dr. Hans-Hermann Francke am Institut für Finanzwissenschaft der Universität Freiburg. 
Öffentliche Sportförderung in der Bundesrepublik Deutschland

Darstellung und finanztheoretische Analyse 


\section{FINANZWISSENSCHAFTLICHE SCHRIFTEN}

Herausgegeben von den Professoren

Albers, Krause-Junk, Littmann, Oberhauser, Pohmer, Schmidt

Band 70

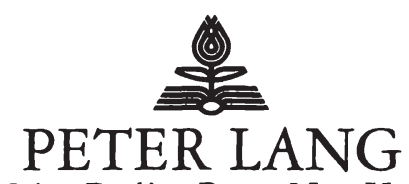

Frankfurt am Main · Berlin · Bern · New York · Paris · Wien 


\section{Christian Hockenjos}

\section{Öffentliche Sportförderung \\ in der \\ Bundesrepublik \\ Deutschland}

Darstellung und

finanztheoretische Analyse

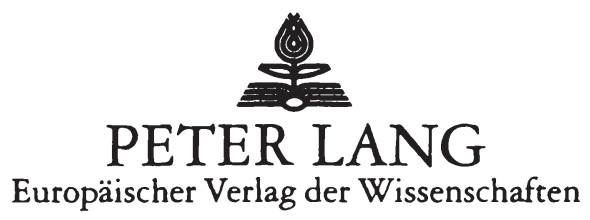

Christian Hockenjos - 978-3-631-75159-6 
Die Deutsche Bibliothek - CIP-Einheitsaufnahme Hockenjos, Christian:

Öffentliche Sportförderung in der Bundesrepublik Deutschland : Darstellung und finanztheoretische Analyse / Christian Hockenjos. - Frankfurt am Main ; Berlin ; Bern ; New York ; Paris ; Wien : Lang, 1995

(Finanzwissenschaftliche Schriften ; Bd. 70)

Zugl.: Freiburg (Breisgau), Univ., Diss., 1995

ISBN 3-631-49173-5

NE: GT

Open Access: The online version of this publication is published on www.peterlang.com and www.econstor.eu under the international Creative Commons License CC-BY 4.0. Learn more on how you can use and share this work: http://creativecommons.org/ licenses/by/4.0.

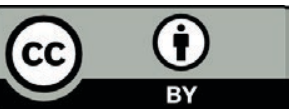

This book is available Open Access thanks to the kind support of ZBW - Leibniz-Informationszentrum Wirtschaft.

D 25

ISSN 0170-8252

ISBN 3-631-49173-5

ISBN 978-3-631-75159-6(eBook)

(C) Peter Lang GmbH

Europäischer Verlag der Wissenschaften

Frankfurt am Main 1995

Alle Rechte vorbehalten.

Das Werk einschließlich aller seiner Teile ist urheberrechtlich geschützt. Jede Verwertung außerhalb der engen Grenzen des

Urheberrechtsgesetzes ist ohne Zustimmung des Verlages unzulässig und strafbar. Das gilt insbesondere für Vervielfältigungen, Übersetzungen, Mikroverfilmungen und die Einspeicherung und Verarbeitung in elektronischen Systemen.

Printed in Germany 123467 
Meiner Freundin Gisela, meinen Eltern und Dietmar 
Christian Hockenjos - 978-3-631-75159-6

Downloaded from PubFactory at 01/11/2019 07:36:54AM

via free access 


\section{Vorwort}

Diese Dissertation ist ein Werk, das mir als begeistertem Sportler und damit großem Nutznießer öffentlicher Sportförderpraxis vereinzelt Gewissensbisse bereitet hat. So galt es selbstverständlich, den Status Quo der Sportförderung kritisch zu hinterfragen. Dabei mußte sich letztlich der neutrale Ökonom gegenüber dem passionierten Sportler durchsetzen, weshalb insbesondere der theoretische Teil der Dissertation nicht ganz frei von kritischen Tönen am Phänomen öffentliche Sportförderung bleiben konnte.

Von weitaus größerer Bedeutung als derartige "Problemchen" waren jedoch die Menschen, die mir in den letzten drei Jahren zur Seite standen und mir die Kraft gaben, diese Aufgabe allererst zu bewältigen. Ihnen möchte ich an dieser Stelle meinen Dank aussprechen.

Neben den Menschen, denen ich diese Arbeit gewidmet habe, gilt mein Dank in erster Linie meinem Doktorvater, Prof. Dr. Hans-Hermann Francke, der mich jederzeit hervorragend betreute und mir neben etlichen Ratschlägen auch stets die nötige Rückendeckung gab.

Von großer Bedeutung war auch das angenehme Klima am Institut für Finanzwissenschaft. In Dr. Jochen Michaelis, der maßgeblich an der Themenstellung beiteiligt war sowie Dr. Alexander Spermann fand ich mehr als kompetente Diskussionspartner und Mentoren.

Für die gleichermaßen notwendige finanzielle Absicherung danke ich neben meinen Eltern abermals Prof. Dr. Hans-Hermann Francke sowie Prof. Dr. Ulrich Sommer und Prof. Dr. Peter Storz (beide Berufsakademie Villingen-Schwenningen), Prof. Dr. Eugen Paul und Prof. Dr. Annemarie Mai (beide Berufsakademie Lörrach) sowie den Rechtsanwälten der Kanzlei Jeutter, Paul und Partner in Freiburg.

Ebenso gilt mein Dank Hartmut Thielen vom Deutschen Städtetag in Köln, der mich bei der Anfertigung meiner Abfrage kommunaler Sportausgaben unterstützte sowie sämtlichen, von mir konsultierten Mitarbeitern von kommunalen Sportämtern, von Sportvereinen und -verbänden, die mir viele wertvolle Tips geben konnten und ohne deren Hilfe die empirischen Teile dieser Arbeit nicht ermöglicht worden wären.

Für die Aufnahme in die Schriftenreihe danke ich dem Mitherausgeber, Prof. Dr. Alois Oberhauser. Ebenso gilt mein Dank dem Koreferenten der Arbeit, Prof Dr. Siegfried Hauser. Beide haben durch abschließende Anregungen dazu beigetragen, das Manuskript weiter zu verbessern. 
Christian Hockenjos - 978-3-631-75159-6

Downloaded from PubFactory at 01/11/2019 07:36:54AM

via free access 


\section{Inhaltsverzeichnis}

Seite

Einleitung: Sportförderung - Gegenstand ökonomischer Analyse? 1

Kap. I Sport und öffentliche Sportförderung - Begriffliche Abgrenzungen 5

Kap. II Die Organisation des Sports in der Bundesrepublik Deutschland 9

1. Sportanbieter in der Bundesrepublik Deutschland 9

2. Trägerschaftsverhältnisse von Sportstätten 13

Kap. III Die öffentliche Sportförderung in der Bundesrepublik Deutschland 17

1. Allgemeine Grundsätze öffentlicher Sportförderung

2. Die Sportförderung durch öffentliche Ausgaben in den Gebietskörperschaften der Bundesrepublik Deutschland

2.1. Die Sportförderung der Kommunen ................................................... 18

2.1.1. Abriß zur Geschichte kommunaler Sportpolitik und -förderung .... 18

2.1.2. Träger kommunaler Sportpolitik .............................................. 19

2.1.3. Legislative Grundlagen - Gesetze, Leitlinien und Verordnungen ... 20

2.1.4. Kommunal-Haushalte und Sportförderung ............................... 21

2.1.4.1. Haushaltsabschnitt 550 - Sportamt und Förderung des Sports

2.1.4.2. Haushaltsabschnitt 560 - Sportstätten ....................... 22

2.1.4.3. Haushaltsabschnitt 570 - Badeanstalten ....................... 23

2.1.4.4. Sportförderung außerhalb des Einzelplanes 5 .............. 24

2.1.5. Sportstättenleitpläne als Instrument kommunaler Sportentwicklungsplanung.

2.1.6. Qualitative Aspekte kommunaler Sportförderung - eine Analyse kommunaler Sportförderrichtlinien und -praxis

2.1.6.1. Die Empfehlungen des Deutschen Städtetages als Grundlage kommunaler Sportförderrichtlinien. 
2.1.6.2. Allgemeine Voraussetzungen für den Erhalt direkter öffentlicher Zuwendungen.

2.1.6.3. Zuschüsse zu Baumaßnahmen der Vereine .................... 34

2.1.6.4. Kriterien zur Überlassung kommunaler Sportstätten ........ 37

2.1.6.5. Pflege und Unterhaltung von Sportstätten....................... 42

2.1.6.6. Zuschüsse zur Anschaffung von Sport- und Platzpflegegeräten ..................................................................... 46

2.1.6.7. Förderung des Spitzensports ……………….................. 46

2.1.6.8. Personelle Förderungsaspekte........................................... 48

2.1.6.9. Ehrungen und Jubiläumsgaben ...................................... 49

2.1.6.10. Die Förderung einzelner Sportarten .................................. 49

2.1.6.11. Gemeindliche Sportförderrichtlinien - ein Instrument zur Vereinheitlichung kommunaler Sportpolitik?............. 50

2.1.7. Exkurs: Kommune und Profifußball ............................................. 51

2.1.8. Quantitative Aspekte kommunaler Sportförderung ........................ 64

2.1.8.1. Die Entwicklung kommunaler Sportausgaben von

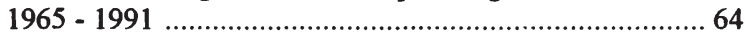

2.1.8.2. Die Sportausgaben der Kommunen im Jahr 1991 im Querschnitt .................................................................. 74

2.2. Die Sportförderung der Länder ......................................................... 79

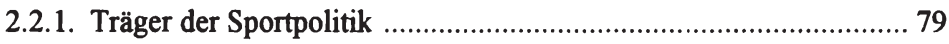

2.2.2. Legislative Grundlagen - Gesetze, Leitlinien und Verordnungen ... 80

2.2.3. Länder-Haushalte und Sportförderung ........................................ 81

2.2.4. Qualitative Aspekte der Sportförderung der Bundesländer ............ 81

2.2.4.1. Unterstützung des kommunalen und vereinseigenen Sportstättenbaus ............................................................... 81

2.2.4.2. Laufende Förderung des Vereinssports .......................... 82

2.2.4.3. Sonstige Förderungsaspekte …………......................... 82

2.2.5. Quantitative Aspekte der Sportförderung der Bundesländer .......... 83

2.2.5.1. Die Entwicklung der Sportausgaben der Bundesländer von 1965 bis 1991 ......................................................... 83

2.2.5.2. Die Sportausgaben der Bundesländer im Jahr $1991 \mathrm{im}$ Querschnitt ................................................................... 86

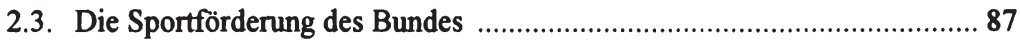

2.3.1. Träger der Sportpolitik ................................................................ 87

2.3.2. Legislative Grundlagen - Gesetze, Leitlinien und Verordnungen ... 87

2.3.3. Sport im Bundeshaushalt ............................................................ 88

2.3.4. Qualitative Aspekte der Sportförderung des Bundes ..................... 88

2.3.5. Quantitative Aspekte - Die Entwicklung der Sportausgaben des Bundes von 1965 bis 1991 ........................................................ 90

2.4. Der Gesamtumfang öffentlicher Sportausgaben ...................................... 91 
3. Öffentliche Sportförderung durch Steuererleichterungen 94

4. Exkurs: Weitere Anmerkungen zum organisierten Sport 96

5. Zusammenfassung der Ergebnisse und Darstellung des Erklärungsbedarfs 98

Kap. IV Theoretische Erklärungsansätze öffentlicher Sportförderung 101

1. Sport und die Theorie der öffentlichen Güter ............................................ 101

1.1. Die Theorie der öffentlichen Güter ................................................. 101

1.1.1. Reine öffentliche Güter und effiziente Allokation ...................... 101

1.1.2. Mischgüter und effiziente Allokation ...................................... 102

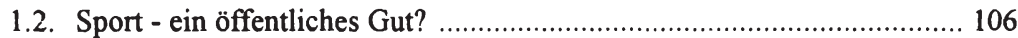

1.2.1. Zur Spezifizierung des Begriffes Sport im Lichte der Kollektiv-

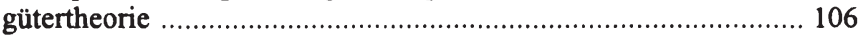

1.2.2. Sportgüter und die Möglichkeit zum Ausschluß - Internalisierte Nutzen vs. externe Effekte des Sports ....................................... 108

1.2.2.1. Kernnutzen von Sportgütern ....................................... 108

1.2.2.2. Externe Effekte des Sports ……................................. 109

1.2.2.2.1. Sport und Gesundheit .................................... 109

1.2.2.2.2. Soziale Aspekte des Sports ............................ 116

1.2.2.2.3. Sport als Standortfaktor ............................... 120

1.2.2.2.4. Sport als Werbeträger .................................... 121

1.2.2.2.5. Sport - die wichtigste Nebensache der Welt ... 125

1.2.2.2.6. Sport und Ökologie ........................................ 127

1.2.3. Sportgüter und Rivalität im Konsum ....................................... 130

1.2.3.1. Aktive Sportausübung ……..................................... 130

1.2.3.2. Der Zuschauersport ................................................... 137

1.2.4. Interpretation der Ergebnisse: Neoklassische Überlegungen als Erklärungsansatz für den Status quo von Sportförderung und die Angebotsstruktur von Sportgütern? ....................................... 138

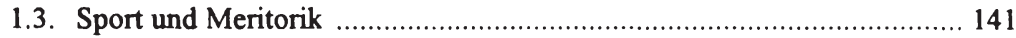

1.3.1. Grundlagen der Meritorik ..................................................... 141

1.3.2. Sport und verzerrte Präferenzen ........................................... 142

1.3.3. Verteilungspolitische Aspekte öffentlicher Sportförderung .......... 144 


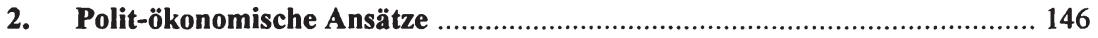

2.1. Die Downs'sche Stimmenmaximierungshypothese ............................... 146

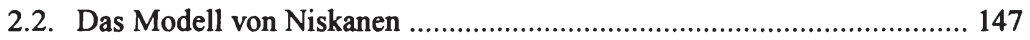

2.3. Sportförderung als Vereinsförderung - Interessengruppentheoretische Ansätze

3. Sportpolitik als Kommunalpolitik - Föderalismustheoretische Gedanken .. 151

3.1. Theoretische Überlegungen zur Vermeidung und Internalisierung

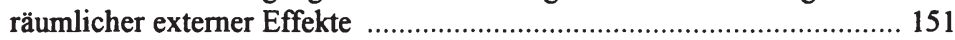

3.2. Sportgüter und rämliche externe Effekte ................................................. 152

3.2.1. Differenzierung nach Nutzenarten ............................................ 153

3.2.2. Differenzierung nach infrastrukturellen Gegebenheiten des Um-

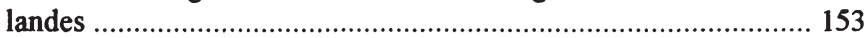

3.2.3. Differenzierung nach verschiedenen Sportgütern ......................... 154

Kap.V Bestimmungsfaktoren öffentlicher Sportbudgets ............................... 157

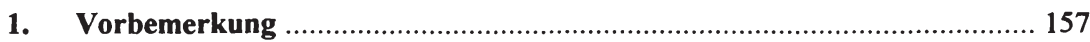

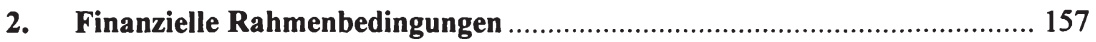

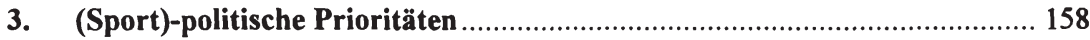

4. Zweckzuweisungen von Bund und Ländern ........................................... 160

5 Sportausgaben als Fixum - Folgekosten und polit-ökonomische

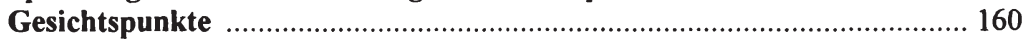

6. Die Subjektivität der Bewertung von Sportgütern........................................ 163

Kap. VI Resümee und Ausblick ………………............................................ 165

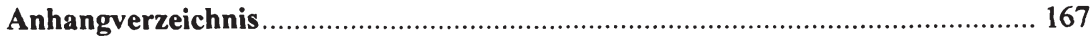

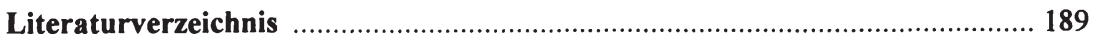




\section{Abbildungsverzeichnis}

Seite

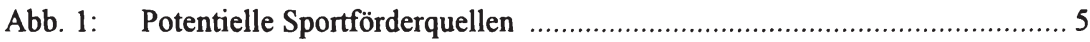

Abb. 2: Spezifizierung des Begriffs öffentliche Förderung ………....................... 6

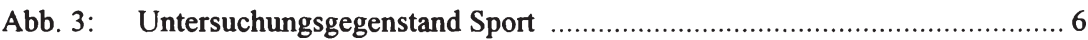

Abb. 4: Marktanteile verschiedener Sportanbieter in der Stadt Karlsruhe ............. 11

Abb. 5: Fördersätze für den Bau vereinseigener Sportstätten ………………......... 36

Abb. 6: Preisdifferenzierung im Rahmen der Bereitstellung kommunaler Sportstätten

Abb. 7: Prozentuale Anteile verschiedener Ausgabepositionen an den Gesamtausgaben für das Mönchengladbacher Bökelbergstadion im Haushaltsansatz 1993

Abb. 8: Kommunale Sportausgaben von 1965 bis 1991 in MIO DM, nominal, getrennt nach verschiedenen Ausgabe- und Einnahmearten

Abb. 9: Kommunale Nettosportausgaben von 1965 bis 1991 in MIO DM, nominal, getrennt nach verschiedenen Haushaltsabschnitten

Abb. 10: Kommunale Nettosportausgaben von 1965 bis 1991 in MIO DM, nominal und real, Basisjahr 1985

Abb. 11: Laufende und investive nominale kommunale Sportausgaben in MIO DM von 1965 bis 1991

Abb. 12: Laufende und einmalige reale Ausgaben für Förderung des Sports und Sportstätten sowie Badeanstalten von 1965 bis 1991 in MIO DM, Basisjahr 1985 70

Abb. 13: Prozentualer Zuschußbedarf bei Ausgaben für Förderung des Sports und Sportstätten sowie Badeanstalten von 1965 bis 1991

Abb. 14: Prozentuale Anteile verschiedener Sportmaßnahmen am GesamtSport-Haushalt 1991, Bereinigte Ausgaben

Abb. 15: Prozentuale Anteile verschiedener Sportmaßnahmen am GesamtSport-Haushalt 1991, Bruttoausgaben

Abb. 16: Die laufenden Bruttoausgaben für Sportförderung und Sportstätten 1991 in MIO DM im Querschnitt

Abb. 17: Die laufenden Bruttoausgaben für Sportförderung und Sportstätten 1991 in DM pro Kopf im Querschnitt 77

Abb. 18: Die laufenden direkten Zuwendungen an Sportvereine 1991 in DM pro Kopf im Querschnitt

Abb. 19: Zuständigkeiten für den Sport in den Landesregierungen der Flächenbundesländer

Abb. 20: Nettosportausgaben der Bundesländer (incl. Stadtstaaten) von 1965 bis 1991 in MIO DM 


\section{XIV}

Abb. 21: Nettosportausgaben der Bundsländer (incl. Stadtstaaten) von 1965 bis 1991 in MIO DM, nominal und real, Basisjahr 1985

Abb. 22: Die Nettosportausgaben der Flächenbundesländer 1991 in DM pro

Kopf im Vergleich 86

Abb. 23: Die Sportausgaben des Bundes von 1965 bis 1991 in MIO DM 90

Abb. 24: Öffentliche Sportförderung in den Gebietskörperschaften der BRD von 1965 bis 1991 in MIO DM

Abb. 25: Nominale und reale Sportförderung in der BRD von 1965 bis 1991 in MIO DM, Basisjahr 1985 93

Abb. 26: Zur Präzisierung des Begriffes Sport 107 


\section{Tabellenverzeichnis}

Seite

Tab. 1: Die Entwicklung des Deutschen Sportbundes von 1965 bis 1993 .............. 9

Tab. 2: Trägerschaftsverhältnisse baden-württembergischer Sportstätten im Jahr 1992

Tab. 3: Trägerschaftsverhältnisse nordrhein-westfälischer Kernsportstätten im Jahr 1988 ........................................................................ 14

Tab. 4: Trägerschaftsverhältnisse nordrhein-westfälischer Sondersportanlagen .... 15

Tab. 5: Mitgliedsbeiträge als Voraussetzung der Zuschußgewährung .................. 32

Tab. 6: Allgemeine Voraussetzungen für den Erhalt direkter öffentlicher Zuwendungen ............................................................................. 33

Tab. 7: Fördersätze für den Bau vereinseigener Sportanlagen ............................. 35

Tab. 8: Regelungen zur Vergabe kommunaler Sportstätten an Sportvereine und Dritte .............................................................................. 40

Tab. 9: Schwimmsportvereine und die Nutzung kommunaler Badeanstalten zu Trainings- und Wettkampfzwecken ............................................. 41

Tab. 10: Zuschüsse zur Pflege von Sportanlagen .............................................. 42

Tab. 11: Unterhaltungszuschüsse für Rasensportflächen und Leichtathletikanlagen

Tab. 12: Gewährung von Unterhaltungszuschüssen zum Betrieb vereinseigener Sondersportanlagen 43

Tab. 13: Zur Heterogenität kommunaler Sportstättenunterhaltungszuschüsse ....... 44

Tab. 14: Zuschüsse zur Anschaffung von Sportgeräten ..................................... 46

Tab. 15: Förderung des Leistungs- und Spitzensports ..................................... 47

Tab. 16: Personenabhängige Förderungen ........................................................ 48

Tab. 17: Zum Ausweis des Freiburger Dreisamstadions im Verwaltungshaushalt der Stadt Freiburg ......................................................................... 53

Tab. 18: Zuschußbedarf zu den laufenden Einnahmen und Ausgaben des Karlsruher Widparkstadions von 1977 bis 1994 in TDM

Tab. 19: Einnahmen, Ausgaben und Kostendeckungsgrad des Stuttgarter Gottlieb-Daimler-Stadions in DM

Tab. 20: Einnahmen, Ausgaben und Kostendeckungsgrad des Mönchengladbacher Bökelbergstadions in DM

Tab. 21: Herz-Kreislauf-Ersterkrankung in 10 Jahren .................................... 113

Tab. 22: Kosten von Sportverletzungen .......................................................... 115

Tab. 23: Werbeträger der Stadt Freiburg ........................................................ 123 
Christian Hockenjos - 978-3-631-75159-6

Downloaded from PubFactory at 01/11/2019 07:36:54AM

via free access 


\section{Abkürzungsverzeichnis}

$\begin{array}{ll}\text { Abb. } & \text { Abbildung } \\ \text { AP'n } & \text { Auskunftsperson } \\ \text { BGH } & \text { Bundesgerichtshof } \\ \text { BMF } & \text { Bundesministerium der Finanzen } \\ \text { BMI } & \text { Bundesministerium des Innern } \\ \text { BSB } & \text { Badischer Sportbund } \\ \text { BVerwG } & \text { Bundesverwaltungsgericht } \\ \text { ch. } & \text { Chapter } \\ \text { c.p. } & \text { ceteris paribus } \\ \text { DOG } & \text { Deutsche Olympische Gesellschaft } \\ \text { DSB } & \text { Deutscher Sportbund } \\ \text { FWT-GmbH } & \text { Freiburger Wirtschafts- und Touristik GmbH } \\ \text { GmbH } & \text { Gesellschaft mit beschränkter Haftung } \\ \text { HK } & \text { Herz-Kreislauf } \\ \text { ISPR } & \text { Internationale Sportrechteverwertungsgesellschaft } \\ \text { K } & \text { Kosten } \\ \text { LSB } & \text { Landessportbund } \\ \text { MIO } & \text { Millionen } \\ \text { MRD } & \text { Milliarden } \\ \text { OVG } & \text { Oberverwaltungsgericht } \\ \text { Tab. } & \text { Tabelle }\end{array}$


Christian Hockenjos - 978-3-631-75159-6

Downloaded from PubFactory at 01/11/2019 07:36:54AM

via free access 


\section{Einleitung: Sportförderung - Gegenstand ökonomischer Analyse?}

Marktwirtschaften sind grundsätzlich dadurch charakterisiert, daß die Ressourcenallokation und -verteilung durch private, wettbewerblich organisierte Unternehmungen erfolgt. So basiert dieses Wirtschaftssystem insbesondere auf der Annahme, daß besagter Unternehmenstypus die von den Individuen nachgefragten Güter am wirtschaftlichsten produzieren und anbieten kann bzw. daß er am ehesten eine effiziente Allokation der Ressourcen herbeiführt.

Hierfür zeichnen primär zwei, der marktwirtschaftlichen Ordnung immanente Mechanismen verantwortlich:

Der Preismechanismus als Informations- und der Wettbewerb als Kontrollinstrument führen innerhalb dieser Wirtschaftsordnung unter anderem zu einem Ausgleich zwischen Angebot und Nachfrage, zu einer Lenkung der Produktionsfaktoren in ihre rentabelste Verwendung, zu einer Rationierung von Anbietern und Nachfragern und schließlich zu einem durch die Eigenverantwortung des Handelns begründeten Leistungsanreiz.

Nun könnte man aus diesen und einigen weiteren, hier nicht erwähnten Charakteristika des marktwirtschaftlichen Ordnungstyps den Schluß ziehen, die Produktion und Bereitstellung von Gütern in dieser Wirtschaftsordnung obliege generell den privaten Unternehmungen.

Ein Blick auf die staatlichen Tätigkeiten innerhalb vieler westlicher Industrienationen zeigt jedoch, daß der öffentliche Sektor eine mitunter nicht zu vernachlässigende Rolle im Wirtschaftsgeschehen einnimmt und daß neben privater auch staatliche und weitere, alternative Formen der Bereitstellung von Gütern existieren.

Hat der Markt also doch gewisse Mängel, oder, anders gefragt, wie lassen sich Staatsquoten bis hin zu etwa $50 \%$ erklären?!

Die Dissertation widmet sich dieser Frage und damit den staatlichen Aktivitäten auf dem Sektor des Wirtschaftsgutes Sport.

Die öffentliche Sportförderung stellt, in absoluten Zahlen ausgedrückt, eine relativ bescheidene Größe dar. Nichtsdestotrotz ist sie eines von vielen Mosaiksteinchen, welche das staatliche Ausgabeverhalten in der Bundesrepublik Deutschland in die oben genannte Größenordnung hat treiben lassen.

Die Analyse eines derartigen Randgebietes öffentlicher Markteingriffe erfährt überdies in Zeiten leerer Staatskassen und einer lebhaft geführten Diskussion bezüglich der Einsparmöglichkeiten öffentlicher Haushalte eine zunehmende Bedeutung.

IVgl. Statistisches Bundesamt, 1993, S. 697. 
Des weiteren handelt es sich beim Phänomen Sport um ein Gut von immenser gesellschaftlicher Bedeutung. Über 24 MIO Mitglieder in Sportvereinen mögen dies eindrucksvoll belegen. ${ }^{2}$ Die nicht verbandsorganisiert Sporttreibenden sind in dieser Zahl noch gar nicht erfaßt. ${ }^{3}$ Diese gesellschaftliche Bedeutung allein macht den Sport zu einem aus volkswirtschaftlicher Sicht interessanten Untersuchungsgegenstand.

Betrachtet man schließlich den Stand der wirtschaftswissenschaftlichen Forschung auf dem Gebiet des Sports, dann zeigt sich folgendes Bild: Der Sport ist, aus ökonomischer Sicht gesehen, bislang ein wissenschaftliches Randgebiet. ${ }^{4}$ Die Sportökonomie ist eine noch recht junge Disziplin wissenschaftlicher Forschung und bisherige Veröffentlichungen auf diesem Gebiet vernachlässigen die Problematik öffentlicher Sportförderung größtenteils.

Die Dissertation möchte sich dieser Problematik annehmen, die öffentliche Sportförderung von finanz- und damit allokationstheoretischer Seite beleuchten und sie dabei gleichermaßen darstellen wie zu erklären versuchen.

$\mathrm{Zu}$ diesem Zweck werden eingangs begriffliche Abgrenzungen vorgenommen, Kapitel II behandelt die Organisation des Sports in der Bundesrepublik Deutschland.

In Kapitel III werden Entwicklung und Status quo der öffentlichen Sportförderung in den Gebietskörperschaften der Bundesrepublik Deutschland dargestellt. Der Schwerpunkt der Betrachtung liegt dabei auf der Sportförderung der Kommunen. Dies hat zwei Gründe. Zum einen besteht öffentliche Sportförderung vorrangig, wie an späterer Stelle gezeigt werden wird, aus kommunaler Sportförderung, zum anderen ist es eben diese Sportförderung der Städte und Gemeinden, derer sich auch die rein deskriptive Literatur bislang recht wenig angenommen hat. ${ }^{5}$

Dieser empirische Teil der Arbeit stützt sich zum Teil auf vom Autor durchgeführte empirische Erhebungen bezüglich qualitativer wie quantitativer Aspekte der öffentlichen Sportförderung. So wurden im Hinblick auf qualitative Gesichtspunkte die Sportförderrichtlinien von 61 deutschen Kommunen unterschiedlicher Größenordnung und

2Diese Zahl bezieht sich auf das wiedervereinigte Deutschland, die Anzahl der Vereinsmitglieder in den neuen Bundesländern ist mit 1,2 MIO allerdings vergleichsweise bescheiden. Vgl. Deutscher Sportbund, 1993.

${ }^{3}$ Eine Studie bezüglich der wirtschaftlichen Bedeutung des Sports ergab, daß sich 70 bis $75 \%$ der Bevölkerung zumindest gelegentlich sportlich betätigen. Vgl. Weber, 1995, S. 43.

${ }^{4}$ Die wissenschaftliche Literatur zum Thema Sport erstreckt sich in erster Linie auf die Trainingslehre, die Sportpädagogik, die Sportpsychologie, die Sportsoziologie sowie die Sportmedizin. Insbesondere die Erkenntnisse der beiden letztgenannten Gebiete wissenschaftlicher Forschung werden innerhalb dieser Dissertation im Rahmen der theoretischen Erörterungen in Kapitel IV in die Diskussion einbezogen.

${ }^{5}$ Ein Großteil der Literatur im Bereich öffentlicher, insbesondere kommunaler Sportfördenung, besteht in unveröffentlichten, kürzeren Essays, Rundschreiben, schriftlich zusammengefaßten Referaten und dergleichen. 
geographischer Lage untersucht. Eine weitere Studie ermittelte die Sportausgaben von über 500 deutschen Kommunen für das Rechnungsjahr 1991. ${ }^{6}$

Grundsätzlich beschränkt sich die Dissertation auf die Gebietskörperschaften der Bundesrepublik vor der Wiedervereinigung. Eine Berücksichtigung der neuen Bundesländer war, wenngleich ebenfalls von großem Interesse, aufgrund der zum Untersuchungszeitpunkt schwierigen Datengewinnung kaum möglich. Darüber hinaus wären etwaige Daten für die Zeit vor der Wiedervereinigung aufgrund der völlig anderen Strukturen des Sports im Wirtschaftssystem der DDR nicht mit den westdeutschen vergleichbar.

Zum Abschluß von Kapitel III werden die wesentlichen Merkmale öffentlicher Sportförderung zusammengefaßt und der Erklärungsbedarf abgeleitet.

Daran anknüpfend wird in Kapitel IV versucht, das Phänomen "Öffentliche Sportförderung" unter Berücksichtigung verschiedener theoretischer Ansätze nachzuvollziehen. Dabei wird zunächst auf neoklassischer Basis der Aspekt der allokativen Effizienz erörtert, wozu auf Erkenntnisse der Theorie der öffentlichen Güter und der in der deutsch-sprachigen Literatur weniger beachteten Klubtheorie zurückgegriffen wird. Die Analyse der externen Effekte und der Rivalitäten im Konsum des Gutes Sports soll dazu dienen, die Frage "Sport - ein öffentliches Gut?" hinreichend zu beantworten.

Die gleichermaßen interessante Fragestellung nach der allokationsoptimalen Aufteilung öffentlicher Sportausgaben auf die verschiedenen Gebietskörperschaften wird ebenfalls in die Betrachtung miteinbezogen, erfährt aufgrund einer unabdingbaren Abgrenzung des Themas allerdings geringeres Gewicht.

Neben diesen neoklassischen werden schließlich auch positive, polit-ökonomische Ansätze zur Erklärung der öffentlichen Sportförderung herangezogen.

Kapitel V erörtert mögliche Bestimmungsfaktoren der Höhe öffentlicher Sportbudgets. $\mathrm{Zu}$ diesem Zweck werden die in Kapitel III dargestellten quantitativen Aspekte öffentlicher Sportausgaben unter Berücksichtigung der in Kapitel IV diskutierten theoretischen Erklärungsansätze analysiert.

Resümee und Ausblick auf mittelfristige Perspektiven schließen die Dissertation mit dem sechsten Kapitel ab.

${ }^{6}$ Vgl. Hockenjos, 1992, S. 278 - 305. 
Christian Hockenjos - 978-3-631-75159-6

Downloaded from PubFactory at 01/11/2019 07:36:54AM

via free access 


\section{Kap. I Sport und öffentliche Sportförderung - Begriffliche Abgrenzungen}

Zum Zweck der exakten Abgrenzung des Untersuchungsgegenstands der Dissertation soll eingangs eine Konkretisierung des Begriffs "Öffentliche Sportförderung" erfolgen. Dabei müssen alle drei Termini berücksichtigt werden, die in diesem Begriff enthalten sind.

Zunächst zum Terminus des Öffentlichen: Wenn im folgenden von "öffentlicher" Sportförderung die Rede ist, dann ist damit ausschließlich die Förderung des Sports von seiten des Staates, also der Gebietskörperschaften gemeint. ${ }^{7}$ Davon abgegrenzt sollen alternative Sportförderquellen seitens der gewerblichen Wirtschaft, insbesondere von Industrie und Handel (Kommerzielles Sportsponsoring) sowie privater Seite oder weiterer denkbarer Herkunft werden.

Abbildung 1: Potentielle Sportförderquellen

\begin{tabular}{|l|l|}
\hline \multirow{2}{*}{ Offentliche Sportförderung durch Staat } & Bund \\
\cline { 2 - 2 } & Länder \\
\cline { 2 - 2 } & Gemeinden \\
\hline Kommerzielles Sportsponsoring durch gewerbliche Wirtschaft & Industrie \\
\cline { 2 - 2 } & Handel \\
\hline Private Sportförderung durch privates Mäzenatentum & private Geldgeber \\
\hline Öffentlich-rechtliche Institutionen & Verbände \\
\cline { 2 - 2 } & sonstige Organisationen \\
\hline
\end{tabular}

Gleichermaßen muß der Terminus "Förderung" spezifiziert werden. Offentliche Förderung des Sports erfolgt auf verschiedene Arten.

Der Staat kann den Sport zum einen fördem, indem er Gelder für die Bereitstellung von Sportgütern oder für die Ausübung von sportlichen Aktivitäten zur Verfügung stellt. Diese Art der öffentlichen Sportförderung durch Ausgaben schlägt sich unmittelbar in den öffentlichen Haushalten nieder.

Die Betrachtung solchermaßen haushaltswirksamer Förderpraktiken macht eine weitere Differenzierung nach den Begriffen "direkte" vs. "indirekte" Förderung erforderlich. Direkte Förderung meint grundsätzlich die Geldervergabe, bspw. für den Bau von Sportstätten. Unter indirekter Förderung versteht man hingegen die Bereitstellung von Gütern. Hierzu zählt in erster Linie die öffentliche Bereitstellung von Sportstätten.

\footnotetext{
${ }^{7}$ Auf eine explizite Betrachtung der Sportförderung seitens der Landkreise wird aufgrund ihrer geringen quantitativen Bedeutung verzichtet.
} 
Sportförderung kann zum anderen in einem Verzicht auf Einnahmen bestehen. So kann der Staat sportliche Aktivitäten fördern, indem er bspw. Steuererleichterungen für Umsätze und Gewinne gewährt, die in diesem Zusammenhang erzielt werden.

Die Dissertation widmet sich in erster Linie den Sportausgaben. Sportförderung durch Einnahmeverzicht fließt am Rande in die Betrachtung mit ein.

Abbildung 2: Spezifizierung des Begriffs öffentliche Förderung
\begin{tabular}{|l|l|l|}
\hline Sportförderung durch & Konkrete Maßnahme \\
\hline Ausgaben & direkte Förderung & Bereitstellung von Geldern \\
\cline { 2 - 3 } & indirekte Förderung & Bereitstellung von Gütern \\
\hline Einnahmeverzicht & Steuererleichterungen \\
\hline
\end{tabular}

Schließlich muß der Begriff "Sport" selbst konkretisiert werden. Läßt sich jede Form der Bewegung als Sport bezeichnen, sind darunter also auch das Wandern und sonstige Erholungsformen zu subsumieren oder ist dieser Begriff enger zu fassen?

Die Arbeit sieht grundsätzlich vor, all jene staatlichen Gelder zu berücksichtigen, die in den öffentlichen Haushalten als Ausgaben für Förderung des Sports und Sportstätten ausgewiesen werden. ${ }^{8}$

Abbildung 3: Untersuchungsgegenstand Sport

\begin{tabular}{|l|l|}
\hline Begriffsabgrenzungen & Untersuchungsgegenstand \\
\hline Ausgaben für Förderung des Sports und Sportstätten & ja \\
\hline Badeanstalten & bedingt \\
\hline Schulsport & nein \\
\hline Sonstige Erholungsformen & nein \\
\hline
\end{tabular}

$\mathrm{Zu}$ den Badeanstalten ist anzufügen, daß sie einen Grenzfall darstellen, werden sie von etlichen Personen doch primär zum Zweck der Erholung und weniger zum Zweck des Sporttreibens besucht. Dies wird umso deutlicher, als in den Ausgaben für Badeanstalten auch die Ausgaben für Saunen, Volksbäder etc. enthalten sind. Daneben werden Badeanstalten von Schwimmsportvereinen aber auch zur aktiven und wettkampforientierten Sportausübung genutzt. Aufgrund dieses partiellen Sportcharakters und nicht zuletzt ihrer großen finanziellen Bedeutung wegen geht dieser Typus öffentlicher Ausgaben am Rande in die Betrachtung mit ein.

Ausgaben im Zusammenhang mit dem Schulsport ${ }^{9}$ und sonstigen Erholungsformen werden grundsätzlich ausgeschlossen.

${ }^{8}$ Diese Begriffsabgrenzung orientiert sich am Ausweis der Sportausgaben in den Kommunalhaushalten. Die gleiche Terminologie findet sich auch in der Reihe "Finanzen und Steuern" des Statistischen Bundesamtes, welche zur Betrachtung der Entwicklung des Sportausgaben herangezogen wurde. 
Auf weitere mögliche Spezifizierungen des Begriffs kann an dieser Stelle zunächst verzichtet werden, da mit der oben genannte haushaltstechnischen Abgrenzung der Untersuchungsgegenstand vorerst hinreichend konkretisiert ist. Weitere Differenzierungen werden nach und nach in die Arbeit miteinbezogen.

${ }^{9}$ Schulsportausgaben, die bspw. für die Gehälter von Sportlehrern anfallen, gehören zum Terrain der Bildungspolitik, welches nicht Thema der Dissertation ist. Eine indirekte Berücksichtigung des Schulsports findet jedoch insofern statt, als Sportstätten des öfteren von Schulen und gleichermaßen von weiteren Sportanbietern genutzt werden. Vgl. hierzu Kapitel III, Abschnitt 2.1.4.4. 
Christian Hockenjos - 978-3-631-75159-6

Downloaded from PubFactory at 01/11/2019 07:36:54AM

via free access 


\section{Kap. II Die Organisation des Sports in der Bundesrepublik Deutschland}

\section{Sportanbieter in der Bundesrepublik Deutschland}

Sportliche Aktivitäten lassen sich auf unterschiedliche Art und Weise anbieten und organisieren.

Vom organisierten Sport wird gesprochen, wenn eine in Sportvereinen organisierte Sportausübung gemeint ist. ${ }^{10}$ Die Vereine als letztes Glied in diesem System organisierter Sportausübung sind in einer mehrstufigen Verbandshierarchie organisiert, an dessen Spitze der Deutsche Sportbund mit Sitz in Frankfurt steht. ${ }^{11}$ Dieser größte deutsche Verband wuchs in der Vergangenheit kontinuierlich an, hatte 1993 allein in den alten Bundesländern über 23 Millionen Mitglieder ${ }^{12}$ und stellt damit alle anderen Interessengruppen in der BRD deutlich in den Schatten. ${ }^{13}$

Tabelle 1: Die Entwicklung des Deutschen Sportbundes von 1965 bis 1993

\begin{tabular}{|l|r|r|r|r|r|r|r|r|}
\hline Jahr & 1965 & 1969 & 1973 & 1977 & 1981 & 1985 & 1989 & 1993 \\
\hline Anzahl der Vereine in Tausend & 34,4 & 38,3 & 41,5 & 46,9 & 58,9 & 61,5 & 66,7 & 69,7 \\
\hline Mitglieder in MIO & 6,8 & 9,6 & 12,2 & 14,7 & 17,7 & 19,3 & 21 & 23,1 \\
\hline Mitglieder in \% der Bevölkerung & 11,7 & 16 & 19,8 & 23 & 28,7 & 31,4 & 34,3 & 37,5 \\
\hline
\end{tabular}

Quelle: Deutscher Sportbund, 1990 und 1993.

Die wichtigsten Gremien des Deutschen Sportbundes sind die Mitgliederversammlung, der Hauptausschuß und das Präsidium samt Hauptverwaltung. Die Mitgliedsorganisationen des DSB bestehen aus über 50 Spitzenverbänden, getrennt nach verschiedenen Sportarten, Sportverbänden mit besonderer Aufgabenstellung sowie einem Netz von Landes-, Bezirks-, Kreis- und Stadtsportbünden. Ebenfalls an dieses System des organisierten Sports angeschlossen sind das Nationale Olympische Komitee, die Deutsche Olympische Gesellschaft sowie die Stiftungen Deutsche Sporthilfe und Sicherheit im Skisport. ${ }^{14}$

Mit kommerziell angebotenem Sport sind in aller Regel Formen sportlicher Aktivitäten gemeint, die von privatwirtschaftlichen Unternehmungen angeboten werden.

Neben der in den letzten Jahren stark angestiegenen Anzahl privater Fitneß- Aerobicund Tanzstudios gehören hierzu auch Bäder und Saunen sowie privatwirtschaftliche

${ }^{10}$ Zur Struktur der Sportvereine in der Bundesrepublik Deutschland, vgl. Timm, 1979.

"Zur Struktur der Sportverbände in der Bundesrepublik Deutschland, vgl. Winkler, 1985, speziell zum Deutschen Sportbund, zum Nationalen Olympischen Komitee und der Stiftung Deutsche Sporthilfe, vgl. Gieseler, 1983.

${ }^{12} \mathrm{Vgl}$. Deutscher Sportbund, 1993.

${ }^{13}$ Zum Vergleich: der Deutsche Gewerkschaftsbund hatte 1990 knapp 8 Millionen Mitglieder. Vgl. Institut der Deutschen Wirtschaft, 1992, S. 139.

${ }^{14}$ Zum Aufbau des organisierten Sports in der BRD, vgl. Anhang 1. 
Angebote in Sportarten wie Tennis, Squash, Badminton, Bowling und Reiten. Handelt es sich insbesondere bei den Fitneß- und Aerobicstudios um neuere Erscheinungsformen des Sports, die vom organisierten Sport bislang kaum aufgegriffen wurden, so bestehen für letztgenannte Sportarten sehr wohl Spitzenverbände unter dem Dach des Deutschen Sportbundes.

Die Anzahl der erwerbswirtschaftlicher Sportanbieter ist mittlerweile auf etwa 11.000 angestiegen, die Umsätze dieser Branche betragen jährlich rund 3,1 MRD DM. ${ }^{15}$ Zum Vergleich: die Anzahl der Sportvereine in der BRD beläuft sich derzeit auf etwa $67.000,16$ das heißt, auf einen kommerziellen Sportanbieter kommen etwa sechs Vereine. Läßt man die weiteren Sportanbieter zunächst außer Betracht, ergibt dies einen Marktanteil des kommerziellen am gesamten Sportangebot in Höhe von $14 \%$.

Weitere Angebotsformen des Sports sind sonstige gemeinnützige Vereine, die Sport außerhalb des Wirkungsbereichs des Deutschen Sportbundes anbieten. ${ }^{17}$

Betriebssportgruppen existieren insbesondere innerhalb größerer erwerbswirtschaftlicher Unternehmen oder auch unter dem Dach öffentlicher Arbeitgeber. Im Gegensatz $\mathrm{zu}$ anderen Sportanbietern beschränken sie ihr Angebot im allgemeinen auf ihre Beschäftigten. Zur Sportausübung greifen sie ebenfalls auf kommunale Sporthallen oder Vereinssportanlagen zurück, sind Sportvereinen angeschlossen oder verfügen über eigene Sportstätten.

Als institutionelle Sportanbieter fungieren in erster Linie die Krankenkassen, deren Gesundheitszentren, die Volkshochschulen, Kirchen, die Arbeiterwohlfahrt, Stadtjugendausschüsse, Sozial- und Jugendbehörden und einige weitere Institutionen. ${ }^{18}$ Die Angebote institutioneller Sportanbieter sind vielfältig und erstrecken sich vom allgemeinen Freizeitsport bis hin zu speziellen Bewegungsformen wie Yoga, autogenem Training oder Wirbelsäulengymnastik.

Völlig abseits jeglicher Vereinsbildung oder sonstiger konventioneller Organisationsform stehen ungebundene Freizeitgruppen, die ihre Angebote auf privater Basis auf offenen Sportanlagen durchführen. Derart frei organisierte Sporttreffs auf Freianlagen wie öffentlichen Grünflächen, Bolzplätzen und Trimm-Dich-Pfaden können bezüglich ihrer Gewichtigkeit lediglich geschätzt werden, sind sie doch in aller Regel nicht registriert und erheben keinerlei Ansprüche auf direkte Sportförderungsmittel. In der Stadt Karlsruhe konnte bspw. beobachtet werden, daß allein im städtischen Schloßgarten mehr oder weniger regelmäßig 20 verschiedene Sportarten ausgeübt werden. ${ }^{19}$

\footnotetext{
${ }^{15}$ Vgl. Weber, 1995, S. 47.

${ }^{16} \mathrm{Vgl}$. Bundesministerium des Innern, 1991, S. 17.

${ }^{17}$ Vgl. Stadt Karlsruhe, 1992, S. 2.

${ }^{18} \mathrm{Vgl}$. Stadt Karlsruhe, 1992, S. $8 \mathrm{ff}$.

${ }^{19} \mathrm{Vgl}$. Stadt Karlsruhe, 1992, S. 12.
} 
Wenngleich weiter oben explizit ausgeschlossen, sollen der Vollständigkeit wegen auch Schule und Hochschule als nicht zu unterschätzende Sportanbieter genannt werden. In der Schule ist der Sportunterricht Pflichtbestandteil des Lehrplans einer jeden Altersstufe, auf Hochschulebene ist es der allgemeine Hochschulsport, in dessen Rahmen generell Sportarten verschiedenster Art angeboten werden.

Bundesweite Statistiken, die neben verbandsorganisierten und kommerziellen auch die Marktanteile der weiteren, oben erwähnten Sportanbieter beinhalten, sind nicht bekannt. Es soll an dieser Stelle deshalb exemplarisch auf eine Untersuchung bezüglich des Sportangebots in der Stadt Karlsruhe zurückgegriffen werden.

\section{Abbildung 4: Marktanteile verschiedener Sportanbieter in der Stadt Karlsruhe ${ }^{20}$}
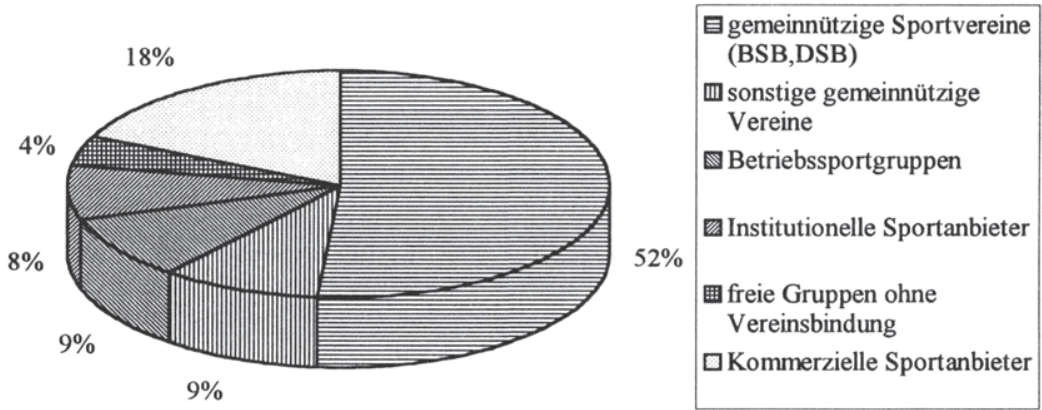

Quelle: Stadt Karlsruhe, 1992, S. 2.

Diese Erhebung aus dem Jahr 1992 zeigt, daß die 187 Karlsruher Sportvereine mit $52 \%$ den Großteil des Sportangebots bereitstellen, daß es daneben aber eine Reihe weiterer, oben beschriebener Anbieter gibt, aus denen 66 kommerzielle Sportanbieter mit einem Marktanteil von $18 \%$ herausragen.

Vergleicht man das Verhältnis von organisiertem zu kommerziellem Sportangebot der Stadt Karlsruhe mit dem der gesamten BRD, dann zeigt sich für die Stadt Karlsruhe ein Verhältnis von $74 \%$ (Vereinssportangebote) zu $26 \%$ (Kommerzielle Sportangebote). Bundesweit hat der Vereinssport also eine stärkere Stellung ( $84 \% \mathrm{zu} 16 \%)^{21}$

${ }^{20}$ Die hier dargestellte Verteilung berücksichtigt die ungebundene Sportausübung freier Gruppen nur unvollständig; konkrete Angaben zur Anzahl dieser freien Sportgruppen können nicht gegeben werden. Auch Schul- und Hochschulsport werden nicht berücksichtigt.

${ }^{21}$ Weiter oben wurde festgehalten, daß 11.000 kommerziellen Anbietern 67.000 Sportvereine gegenüberstehen. 
was - die Karlsruher Verhältnisse als repräsentativ vorausgesetzt - dahingehend interpretiert werden könnte, daß sich kommerzielle Anbieter eher in den größeren Kommunen ausbreiten dürften.

Zusammenfassend kann festgehalten werden, daß die Sportangebotsstruktur in der BRD vom organisierten Sport dominiert wird, daß sich mit den kommerziellen Anbietern jedoch eine zweite, ebenfalls bedeutsame Anbietergruppe am Markt etabliert hat. Im weiteren Verlauf der Arbeit wird immer dort, wo verschiedene Sportanbieter in die Betrachtung einfließen, das Hauptaugenmerk ihrer relativen Bedeutung entsprechend auf diese beiden Sportanbieter gelegt.

Fragt man nach Entwicklungen und Tendenzen im Bereich der unterschiedlichen Sportanbieter, dann zeigt sich folgendes Bild: Der unter dem Dach des DSB organisierte Vereinssport ist der bedeutendste Sportanbieter in der BRD und wird es wohl auch auf unbestimmte Zeit bleiben.

Gleichzeitig klagen jedoch etliche Vereine über Mitgliedsschwund insbesondere innerhalb der sportlich eher aktiven Jugendabteilungen. Als gutes Beispiel dient der in der BRD so populäre Fußballsport. Viele Fußballvereine in kleineren Gemeinden monieren, daß sie nicht mehr genügend Jugendliche für den Fußballsport im Verein gewinnen könnten und zur Bildung von Spielergemeinschaften mit benachbarten Ortschaften gezwungen seien. Mögliche Gründe hierfir sind der rapide Anstieg alternativer sportlicher und auch sonstiger Freizeitbetätigungen und eventuell auch ein genereller Wandel der Bedürfnisstruktur hin zu ungebundeneren Ausübungsformen des Sports, bspw. unter dem Dach kommerzieller Anbieter oder in freien Gruppen. ${ }^{22}$

Die zukünftige Entwicklung der Sportangebotsstruktur wird möglicherweise auch davon abhängen, inwieweit die Sportvereine die Entwicklungstendenzen im Freizeitsportsektor aufnehmen und ihr Programm durch eine Ausdehnung ihrer Angebote an eigenen Fitneßstudios, Gymnastik-, Tanz- und generell ungebundeneren Angeboten ausweiten werden. Die theoretischen Ausfuihrungen greifen die Frage nach den Gründen der dargestellten Sportangebotsstruktur abermals auf und beschäftigen sich dabei insbesondere mit dem Verhältnis zwischen organisiertem und kommerziellem Sport.

22Dies läßt sich aus den hohen Zuwachsraten kommerzieller Anbieter in den vergangenen Jahren ablesen, vorausgesetzt, es handelt sich nicht um vorübergehende Modeerscheinungen. 


\section{Trägerschaftsverhältnisse von Sportstätten}

Eine Frage, die unabhängig vom jeweiligen Sportanbieter zu beantworten ist, ist jene nach den Trägerschaftsverhältnissen von Sportstätten. Sportanbieter und Träger einer Sportstätte müssen keineswegs identisch sein, so bspw., wenn ein Verein Sport auf einer kommunalen Sportanlage anbietet.

Die Betrachtung der Trägerschaftsverhältnisse von Sportstätten in Baden-Württemberg wie auch in Nordrhein-Westfalen zeigt folgendes, ähnliches Bild. 84,3\% der badenwürttembergischen und gar $90 \%$ der nordrhein-westfälischen Turn- und Sporthallen befinden sich in kommunaler Trägerschaft.

Tabelle 2: Trägerschaftsverhältnisse baden-württembergischer Sportstätten im Jahr 1992

\begin{tabular}{|l|l|l|l|l|l|l|l|l|l|}
\hline Art der Anlage & $\begin{array}{l}\text { Sportanla- } \\
\text { gen insg. }\end{array}$ & \multicolumn{2}{|c|}{ kommunal } & \multicolumn{2}{|c|}{$\begin{array}{l}\text { Vereins- } \\
\text { anlagen }\end{array}$} & \multicolumn{2}{|c|}{ staatlich } & \multicolumn{2}{c|}{$\begin{array}{c}\text { sonstige } \\
\text { Träger }\end{array}$} \\
\hline & & absolut & in $\%$ & absolut & in \% & absolut & in \% & absolut & in \% \\
\hline Turn- u. Sporthallen & 5023 & 4235 & 84,3 & 569 & 11,3 & 87 & 1,7 & 132 & 2,6 \\
\hline Hallenbäder & 714 & 623 & 87,2 & 19 & 2,6 & 16 & 2,2 & 56 & 7,8 \\
\hline Frei- u. Naturbäder & 565 & 522 & 92,4 & 22 & 3,8 & 0 & 0 & 21 & 3,7 \\
\hline Sportplätze & 8034 & 4500 & 56 & 3373 & 41,9 & 71 & 0,9 & 90 & 1,1 \\
\hline Tennisfreianlagen & 2027 & 58 & 2,8 & 1765 & 87,1 & 0 & 0 & 204 & 10 \\
\hline Tennishallen & 506 & 6 & 1,1 & 211 & 41,7 & 0 & 0 & 289 & 57,1 \\
\hline Squashhallen & 172 & 0 & 0 & 11 & 6,4 & 0 & 0 & 161 & 93,6 \\
\hline Reitsportanlagen & 842 & 8 & 1 & 623 & 74 & 0 & 0 & 211 & 25,1 \\
\hline Schießsportanlagen & 1220 & 8 & 0,6 & 1204 & 98,7 & 0 & 0 & 8 & 0,6 \\
\hline Kegelsportanlagen & 940 & 125 & 13,2 & 224 & 23,8 & 0 & 0 & 591 & 62,9 \\
\hline
\end{tabular}

Quelle: Lőw, Sportstătten in Baden-Württemberg, in: Statistisches Landesamt Baden-Württemberg, Monatszeitschrift Baden-Württemberg in Wort und Zahl, 2, 1993, S. 60 - 67.

Anders ist es hingegen bei den Sportplätzen. Zwar sind auch hier die Kommunen Träger von mehr als der Hälfte der gesamten Sportplätze, fast genauso viele Anlagen befinden sich jedoch in Vereinseigentum.

Diese Aufteilung der Trägerschaft von Sportplätzen ist ein jüngeres Phänomen. Hier hat innerhalb der letzten Jahre eine gravierende Veränderung stattgefunden, befanden sich 1980 doch immerhin noch $89 \%$ der Sportplätze in kommunaler Trägerschaft. ${ }^{23}$

${ }^{23}$ Vgl. Haass, 1990, S. 240. 
Tabelle 3: Trägerschaftsverhältnisse nordrhein-westfälischer Kernsportstätten im Jahr 1988

\begin{tabular}{|l|l|l|}
\hline Anlagenart & Kommunal & Verein \\
\hline Sporthallen & $90 \%$ & $3 \%$ \\
\hline Freibäder & $88 \%$ & $7 \%$ \\
\hline Hallenbäder & $83 \%$ & $0 \%$ \\
\hline Sportplätze & $50 \%$ & $43 \%$ \\
\hline
\end{tabular}

Quelle: Der Kultusminister NRW: Sportstăttenstatistik für das Land Nordrhein-Westfalen 1988, Düsseldorf, 1988.

Insgesamt kann festgehalten werden, daß Kernsportstätten fast ausschließlich von Kommunen oder Vereinen betrieben werden.

Ein ganz anderes Verhältnis ergibt hingegen die Betrachtung der Sondersportanlagen..$^{24}$ Kommunen treten nur selten als Träger derartiger Sportstätten auf, vielmehr sind es Vereine oder kommerzielle Anbieter, die solche Sportanlagen führen.

Tennisfrei- sowie Reit- und Schießsportanlagen finden sich primär in Vereinsregie, obschon ein Blick auf die Entwicklung in Nordrhein-Westfalen zeigt, daß auch hier ein leichter Trend hin zu kommerziellen Trägerschaften erkennbar ist. (vgl. Tabelle 4) So hat sich der Anteil kommerzieller Trägerschaften bei Golfplätzen, Reit- und Eissportanlagen, Tennis- und Squashhallen erhöht. Lediglich im Zusammenhang mit Schieß- und Wassersportanlagen ist der Anteil kommerzieller Trägerschaften leicht gesunken, was mit einer Erhöhung kommunaler Trägerschaften in diesen Bereichen einherging.

Bei den Tennishallen hingegen und auch bei den Squashhallen dominieren eindeutig kommerzielle Trägerschaften. 1988 befanden sich $94 \%$ aller Squashhallen in kommerzieller Trägerschaft.

${ }^{24}$ Von den gemäß "Goldenem Plan" als Kernsportstätten definierten Sporthallen, Badeanstalten und Sportplätzen werden spezielle oder Sondersportanlagen wie u.a. Golf- und Tennisplätze, Squashhallen, Schießsport-, Reitsport-, Wassersport- und Eissportanlagen unterschieden. 
Tabelle 4: Trägerschaftsverhältnisse nordrhein-westfälischer Sondersportanlagen

\begin{tabular}{|c|c|c|c|c|c|c|}
\hline Anlageart & \multicolumn{2}{|c|}{ Kommunal (in \%) } & \multicolumn{2}{|c|}{ Verein (in \%) } & \multicolumn{2}{|c|}{ Kommerziell (in \%) } \\
\hline Jahr & 1980 & 1988 & 1980 & 1988 & 1980 & 1988 \\
\hline Golfplätze & 3 & 2 & 95 & 92 & 3 & 6 \\
\hline Wassersportanlagen & 8 & 19 & 62 & 54 & 26 & 20 \\
\hline Reitsportanlagen & 3 & 1 & 70 & 61 & 23 & 32 \\
\hline Tennishallen & 3 & 1 & 28 & 28 & 68 & 69 \\
\hline Squashhallen & 2 & 0 & 6 & 5 & 91 & 94 \\
\hline Reitsportanlagen & 3 & 1 & 70 & 61 & 23 & 32 \\
\hline Schießsportanlagen & 9 & 10 & 79 & 76 & 9 & 8 \\
\hline
\end{tabular}

Quelle: Der Kultusminister NRW: Sportstăttenstatistik für das Land Nordrhein-Westfalen 1988, Düsseldorf 1988.

Zusammenfassend kann festgehalten werden, daß sich Kernsportstätten primär in der Hand von Kommunen oder Vereinen befinden, daß sich bei einigen Sondersportanlagen jedoch Vereine und kommerzielle Anbieter gleichermaßen als Träger hervortun. 
Christian Hockenjos - 978-3-631-75159-6

Downloaded from PubFactory at 01/11/2019 07:36:54AM

via free access 


\section{Kap. III Die öffentliche Sportförderung in der Bundesrepublik Deutschland}

\section{Allgemeine Grundsätze öffentlicher Sportförderung}

Als Grundsätze öffentlicher Sportförderung werden von sportpolitischer Seite vorrangig drei Punkte genannt: die Autonomie des Sports, die Subsidiarität der Sportförderung und die partnerschaftliche Zusammenarbeit. ${ }^{25}$

Ersterer besagt, daß die Selbstverwaltung des Sports bzw. dessen Organisationen ihre Angelegenheiten in eigener Verantwortung regeln und autonom entscheiden, wobei sie bei der Verwendung öffentlicher Gelder bzw. der Inanspruchnahme alternativer Förderungspraktiken den gesetzlichen Vorschriften unterliegen. In dieser Wortwahl zeigt sich deutlich die Orientierung dieses Grundsatzes hin zum organisierten Sport. So ist bspw. im siebten Sportbericht der Bundesregierung nachzulesen, daß die Verfassungsordnung der BRD dem in Vereinen und Verbänden organisierten gesellschaftlichen Bereich einen weiten, grundrechtlich abgesicherten Freiheitsraum gewährt; ${ }^{26}$ der kommerziell angebotene Sport wird in diesem Zusammenhang nicht erwähnt.

Der Grundsatz der Subsidiarität öffentlicher Sportförderung verleiht der Sportförderung den Charakter einer Hilfe zur Selbsthilfe. So ist eine allgemeine Voraussetzung für die Gewährung öffentlicher Gelder das Vorhandensein von Eigenmitteln. Zuschüsse der öffentlichen Hände können in dem Maße gewährt werden, in dem die eigenen Mittel nicht ausreichen. Unter eigenen Mitteln in diesem Sinne wird nicht nur eigenes Kapital, sondern auch die Einbringung ehrenamtlicher Tätigkeit verstanden, wie sie vor allem im Sportstättenbau und in der laufenden Unterhaltung der Sportanlagen und des Sportbetriebes von Sportvereinen eine große Bedeutung erfährt.

Verbleibt der Grundsatz der partnerschaftlichen Zusammenarbeit zwischen Staat und Sport. Dieser Grundsatz sieht eine Zusammenarbeit zwischen staatlichen Gremien und Selbstverwaltung des Sports vor, die sich von der Abstimmung im Konzeptionellen über die Erarbeitung gemeinsamer Vorstellungen bis hin zu Beteiligungen des Sports an Delegationen bei internationalen Konferenzen erstreckt.

Es soll Aufgabe der kommenden Abschnitte sein, die auf diesen Grundsätzen basierende Sportpolitik der Gebietskörperschaften näher zu beleuchten. $\mathrm{Zu}$ diesem Zweck wird, ihrer Bedeutung angemessen, mit der Sportförderung der Kommunen begonnen.

\footnotetext{
${ }^{25}$ Vgl. U.a. Bundesministerium des Innern, 1991, S. 19 ff., Deutscher Städtetag, 1987, Deutscher Sportbund, 1991 und Gieseler 1983, S. 110.

${ }^{26} \mathrm{Vgl}$. Bundesministerium des Innern, 1991, S. 19.
} 


\section{Die Sportförderung durch öffentliche Ausgaben in den Gebietskörper- schaften der Bundesrepublik Deutschland}

\subsection{Die Sportförderung der Kommunen}

\subsubsection{Abriß zur Geschichte kommunaler Sportpolitik und -förderung}

Sehr bald nach der Gründung des Deutschen Sportbundes am 10.12.1950 trat die Interessenvertretung des organisierten Sports mit Überlegungen hervor, den Bedarf an einzelnen Sportstättenarten durch Orientierungswerte in Abhängigkeit von der Einwohnerzahl festzulegen. Bereits 1956 brachte die Deutsche Olympische Gesellschaft die in Zusammenarbeit mit den kommunalen Spitzenverbänden erarbeiteten Richtlinien zur Schaffung von Erholungs-, Spiel- und Sportanlagen, "DOG-Richtlinien", heraus, ${ }^{27}$ die die damals bundesweit erhebliche Bedarfslücke im Bestand von Sportstätten aufzeigte. So waren nach dem Krieg mehr als $40 \%$ aller Sportanlagen zerstört, bei den Sporthallen waren es gar $60 \% .28$

Zunächst wurden die DOG-Richtlinien für Gemeinden mit 5.000 und mehr Einwohnern entwickelt, bereits 1959 jedoch für Gemeinden mit weniger als 5.000 Einwohnern ergänzt. ${ }^{29}$ Inhalt dieser Richtlinien waren einheitliche Grundwerte für den Sportstättenbau in Gemeinden aller Größenordnungen. Nach der zweiten Fassung im Jahre 1967 entstand 1976 die noch heute gültige, dritte Fassung, welche unter anderem je nach Einwohnerzahl pauschalierte Flächenvorgaben für die Feststellung des örtlichen Sportbedarfs enthält. ${ }^{30}$

Der "Goldene Plan"31 der Deutschen Olympischen Gesellschaft aus dem Jahr 1959 war ein auf den oben genannten Richtlinien aufbauender Plan, die Bedarfslücken an sportlicher Infrastruktur in der BRD binnen 15 Jahren zu schließen.

Der Plan sah im einzelnen vor: 31.000 Kinderspielplätze, 14.700 Schulsportplätze, 10.400 Turn-, Spiel- und Gymnastikhallen, 5.500 Gymnastikhallen bzw. -räume, 2.625 Lehrschwimmhallen, 2.420 Freibäder und 435 Schwimmhallen zu errichten.

Ursprünglich war für die Realisierung des Goldenen Plans ein finanzieller Rahmen von 6,3 MRD DM geplant. Letztlich belief sich der Gesamtaufwand auf 17,4 MRD DM, wovon die Gemeinden im Endeffekt nicht wie vorgesehen 30, sondern $63 \%$ trugen.

${ }^{27}$ Vgl. Deutsche Olympische Gesellschaft, 1956.

${ }^{28}$ Vgl. Gieseler, 1983, S. 26.

${ }^{29} \mathrm{Vgl}$. Mombaur/ Mattner-Stellmann, 1988, S. 5.

30Vgl. Deutsche Olympische Gesellschaft (DOG): Richtlinien für die Schaffung von Erholungs-, Spielund Sportanlagen - neue III. Fassung, Frankfurt a.M., 1976, und auch Abschnitt 2.1.5.

${ }^{31} \mathrm{Vgl}$. Deutsche Olympische Gesellschaft, 1959. 
Ein Goldener Plan Ost existiert heute bereits in der zweiten Fassung mit dem Ziel der Schaffung einer vergleichbaren sportspezifischen kommunalen Infrastruktur in den neuen Bundesländern. ${ }^{32}$

Parallel zu dieser, entscheidend von seiten des organisierten Sports mitinitiierten Politik einer Förderung des Sports, forcierten die kommunalen Spitzenverbände ihre Bemühungen, die bis dahin noch sehr uneinheitliche Sportpolitik der Kommunen nicht zu nivellieren, so aber doch bezüglich ihrer Transparenz und Qualität einander anzugleichen. Damit angesprochen sind die kommunalen Sportförderrichtlinien, deren Geschichte und Entwicklung kurz skizziert werden soll.

Bereits 1958 hatte der Deutsche Städtetag Leitsätze für die kommunale Sportpflege herausgegeben, ${ }^{33}$ die in den 60er Jahren - vorrangig in größeren Städten - zu einem ersten Schub von kommunalen Sportförderrichtlinien führten; damals allerdings überwiegend ohne ablesbare Festsetzungen. ${ }^{34}$ In den 70er Jahren wurden diese Förderungsrichtlinien zunehmend konkretisiert und entwickelten sich damit zu einer Planungsgrundlage vorrangig für die Sportvereine einer Kommune. Im vergangenen Jahrzehnt erfolgten nicht zuletzt auf Druck der Sportorganisationen und -vereine weitere Ausdifferenzierungen und Verfeinerungen der einzelnen Förderungsaspekte. ${ }^{35}$

\subsubsection{Träger kommunaler Sportpolitik}

Entscheidungen über Art und Ausmaß der Förderung des Sports werden auf kommunaler Ebene in den gemeindlichen Verwaltungen vorbereitet und letztlich von den Gemeinderäten getroffen. Die Kommunen bedienen sich dabei, je nach Größe, unterschiedlich umfangreicher sportpolitischer Verwaltungsinstanzen. Größere Städte verfügen zumeist über ein eigens eingerichtetes Sportamt oder gar -dezernat, welches die Angelegenheiten des Sports verwaltetet. ${ }^{36}$ Zuweilen werden die Angelegenheiten des Sports auch in sogenannten Schul- und Sportämtern, Sozial-, Jugend- und Sportämtern oder auch Kultur- und Sportämtern verwaltet. ${ }^{37}$ In kleineren Kommunen mit weniger

\footnotetext{
${ }^{32} \mathrm{Vgl}$. Deutscher Sportbund, 1993a.

${ }^{33} \mathrm{Vgl}$. Deutscher Städtetag, 1958.

${ }^{34} \mathrm{Vgl}$. Stadler, 1993.

${ }^{35}$ Vgl. Stadler, 1993, S.I.

36Die Auswertung der weiter oben bereits kurz angesprochenen Abfrage der Sportausgaben aller Kommunen mit mehr als 20.000 Einwohnern zeigte, daß fast alle Gemeinden ab einer Größenordnung von etwa 35.000 Einwohnern eigene Sportämter führen.

37Diese Organisationsform der Sportverwaltung zeigte sich im Großteil jener Kommunen mit 20.000 bis etwa 35.000 Einwohnern.
} 
differenzierten Aufbauorganisationen obliegt der Sport zumeist dem Hauptamt, welches Fragen auf diesem Sektor im Rahmen seiner Aufgaben mitverwaltet. ${ }^{38}$

Als indirekte Träger oder zumindest Orientierungshilfe für die kommunale Sportpolitik dienen die kommunalen Spitzenverbände. Dies sind der Deutsche Städtetag als Interessenvertreter der Kommunen in einer Größenordnung von über 50.000 Einwohnern und der Deutsche Städte- und Gemeindebund als Verband der kleineren Kommunen. Erstgenannter hat einen eigenen Sportausschuß eingerichtet, dem neben dem DSB auch Länder- und ein Bundesvertreter als ständige Gäste angehören.

Als weiterer indirekter Träger der Sportpolitik fungiert die Deutsche Sportkonferenz. In diesem Gremium, welches gleichzeitig als gemeinsames Sprachrohr von Politik und Sportorganisationen angesehen werden kann, sitzen 12 Vertreter des Sports, ein Vertreter vom Bund, vier von den Ländern, drei von den Gemeinden und schließlich sind auch die politischen Parteien mit vier Gesandten in dieser Konferenz vertreten. Hierin, wie auch in der Tatsache, daß bspw. dem Sportausschuß des Deutschen Städtetages Vertreter des Sports angehören, zeigt sich abermals die enge Verstrickung zwischen Trägern der Sportpolitik und der Interessenvertretung des organisierten Sports. ${ }^{39}$

\subsubsection{Legislative Grundlagen - Gesetze, Leitlinien und Verordnungen}

Das Aufgabengebiet der Kommunen läßt sich primär auf das Grundgesetz zurückführen. Art. 28 Abs. 2 GG gewährleistet den Gemeinden, alle Belange der örtlichen Gemeinschaft im Rahmen der Gesetze in Eigenverantwortung zu regeln.

Dies stellt freilich keine spezifizierte gesetzliche Grundlage für den Bereich des Sports dar, dergleichen läßt sich auch in keinerlei Gesetzen finden. Vielmehr sind es Richtlinien verschiedener Art und Herkunft, die die Sportpolitik der Kommunen prägen. In diesem Zusammenhang sind vor allem die kommunalen Sportförderrichtlinien zu erwähnen, die von der Mehrheit, insbesondere der größeren Gemeinden, erlassen wurden.

Bevor im Rahmen von Abschnitt 2.1.5. die konkreten Bestandteile derartiger Sportförderrichtlinien etwas näher beleuchtet werden, soll zunächst die haushaltstechnische Handhabung kommunaler Sportausgaben dargestellt werden.

\footnotetext{
${ }^{38}$ So vor allem in Gemeinden mit knapp über 20.000 Einwohnern. Es wird unterstellt, daß Gemeinden mit weniger als 20.000 Einwohnern gleichermaßen verfahren.

${ }^{39}$ Diese Verstrickung geht soweit, daß der DSB de facto die Sportpolitik der Kommunen mitzugestalten versucht. So artikuliert er seine Interessen gegenüber den Trägern der Sportpolitik ganz deutlich und empfiehlt bspw. den Kommunen, Umfang und Förderung des Vereinssports mögen zumindest $0,5 \%$ des kommunalen Gesamthaushaltes betragen. 25\% davon seien für den Bau und die Unterhaltung vereinseigener Sportstätten vorzusehen. Vgl. Deutscher Sportbund, 1982, S. 42.
} 


\subsubsection{Kommunal-Haushalte und Sportförderung}

Ausgaben zur Förderung des Sports werden in den Kommunalhaushalten sowohl im Verwaltungs- als auch im Vermögenshaushalt im Einzelplan 5, Gesundheit, Sport und Erholung aufgeführt. ${ }^{40}$ Bezüglich des Sports sind innerhalb dieses Einzelplanes 5 drei Abschnitte von Bedeutung. Es sind dies im einzelnen:

550 Sportamt und Förderung des Sports,

560 Sportstätten sowie

570 Badeanstalten.

Diese drei Positionen unterscheiden sich in erster Linie dadurch, daß erstgenannter auch direkte Zuwendungen an die Sportanbieter enthält, die beiden letzteren hingegen einen gewissen infrastrukturbereitstellenden Charakter besitzen und somit de facto als indirekte Zuwendungen an die Sporttreibenden angesehen werden können.

\subsubsection{Haushaltsabschnitt 550 - Sportamt und Förderung des Sports}

\section{a) Der Verwaltungshaushalt}

Der Abschnitt 550 erfaßt im Verwaltungshaushalt auf der Ausgabeseite die Aufwendungen der Sportverwaltung, das heißt Personal- und Sachausgaben für die allgemeine Verwaltung der Sportangelegenheiten. Hierzu gehören im einzelnen Gehälter, allgemeiner Bürobedarf, kleinere Beschaffungen, Unterhaltung von Einrichtungen und Geräten, Mieten und Pachten, Mitgliedsbeiträge wie bspw. an die Arbeitsgemeinschaft Deutscher Sportämter und dergleichen.

Die Förderung des Sports beinhaltet Zuschüsse an Sportvereine zur Pflege von Sportanlagen, zur Durchführung von Veranstaltungen, zur Beschäftigung von Übungsleitern, bestimmte personenabhängige Zuschüsse, Schuldendiensthilfen, Beiträge und Zuschüsse an Sportverbände sowie Aufwendungen für Ehrungen und Preise. Ein Teil der Kommunen weist innerhalb dieser Position auch kalkulatorische Wertansätze für die Überlassung von Sportstätten aus. ${ }^{41}$

Auf der Einnahmeseite sind in diesem Abschnitt Teilnehmergebühren, Spenden, mögliche Erstattungen von Zuschüssen an Sportvereine und einigen kleinere, zumeist unter dem Begriff "vermischte Einnahmen" zusammengefaßte Erträge zu nennen.

${ }^{40} \mathrm{Zu}$ den Bestandteilen von Haushaltsplänen, vgl. $\S 80 \mathrm{GemO}$, zitiert nach Kunze 1989, S. $138 \mathrm{ff}$. und $\S$ 1 - 6 GemHVO.

${ }^{41} \mathrm{Vgl}$. u.a. die Haushaltspläne der Städte Stuttgart, Karlsnuhe oder Lahr. Die Stadt Mōnchengladbach macht von dieser Möglichkeit ebenfalls Gebrauch, beschränkt sich dabei allerdings auf die Badeanstalten. 


\section{b) Der Vermögenshaushalt}

Einmalige Ausgaben für die Förderung des Sports fallen insbesondere im Zusammenhang mit Zuschüssen zu Bauvorhaben der Vereine an. Weitere Aufwandsposten sind die Gewährung von Darlehen und auch der Erwerb von beweglichen Sachen des Anlagevermögens.

Einnahmen entstehen im Abschnitt 550 relativ selten, soweit vorhanden werden innerhalb dieser Position Darlehensrückflüsse oder aber Zuschüsse von privaten Unternehmen verbucht.

\subsubsection{Haushaltsabschnitt 560 - Sportstätten}

Der Haushaltsabschnitt Sportstätten wird von etlichen Kommunen sowohl im Verwaltungs- als auch im Vermögenshaushalt weiter in Unterabschnitte unterteilt. Diese Gemeinden unterscheiden zwischen Freiluftanlagen und Hallen oder richten für bestimmte Sportstätten wie bspw. ein großes Stadion einen eigenen Unterabschnitt ein.

\section{a) Der Verwaltungshaushalt}

Innerhalb des Abschnitts 560 werden die mit der Unterhaltung kommunaler Sportstätten zusammenhängenden Ausgaben ausgewiesen. Es sind dies vorrangig Ausgaben zur Unterhaltung der Grundstücke und baulichen Anlagen, zur Bewirtschaftung und Reinigung derselben, anfallende Mieten und Pachten sowie verschiedene Versicherungen. Vom Ausweis kalkulatorischer Abschreibungen und Zinsen auf das Anlagekapital wird im Zusammenhang mit kommunalen Sportstätten nur selten Gebrauch gemacht. ${ }^{42}$ Einnahmen erwachsen den Gemeinden in Form von Mieten und Pachten, die von den jeweiligen Nutzern für die Überlassung der Sportstätten entrichtet werden. Daneben entstehen zum Teil Einnahmen aus Verkaufsgenehmigungen und Werbeerträgen.

\section{b) Der Vermögenshaushalt}

Im Vermögenshaushalt werden alle Ausgaben im Zusammenhang mit dem Bau kommunaler Sportstätten erfaßt. Hierunter fallen der Erwerb unbebauter oder auch bebauter Grundstücke, der Erwerb beweglicher Sachen sowie Erschließungs- und Kanalbeiträge, Hochbaumaßnahmen und weitere bauliche Verbesserungen.

\footnotetext{
${ }^{42}$ Die Frage des Ausweises derartiger Aufwandsposten hängt keineswegs grundsätzlich von der Größe einer Kommune ab und differiert mitunter von Unterabschnitt zu Unterabschnitt. Karlsruhe oder Freiburg bspw. weisen kalkulatorische Abschreibungen und Zinsen nur für jeweils einen Unterabschnitt aus. In Karlsruhe ist dies die Europa-, in Freiburg die Eishalle. Stuttgart verzichtet hinsichtlich der Vereinsund Bezirkssportanlagen auf einen derartigen Ausweis, die Stadt Lahr hingegen macht von dieser Möglichkeit grundsätzlich Gebrauch. Vgl. Haushaltspläne der Städte Stuttgart, Karlsruhe, Freiburg und Lahr.
} 
Der größte Einnahmeposten besteht hierbei generell aus Zuwendungen seitens der Bundesländer, mitunter auch des Bundes, die für die Errichtung von Sportstätten gewährt werden.

\subsubsection{Haushaltsabschnitt 570 - Badeanstalten}

\section{a) Der Verwaltungshaushalt}

Der Abschnitt 570 im Verwaltungshaushalt ist Sammelbecken aller laufenden Kosten, die durch die Unterhaltung kommunaler Badeanstalten hervorgerufen werden. Grundsätzlich wird dabei unterteilt zwischen den Personalausgaben, der Unterhaltung der Gebäude, der technischen Anlagen, der Schwimmbecken, der gärtnerischen Anlagen sowie den Ausgaben für sonstige Einrichtungen. Weitere, in Verbindung mit dem Unterhalt von Badeanstalten entstehende Kosten sind die abzuführende Mehrwertsteuer und einige weitere, hier nicht näher aufgeführte kleinere Positionen.

Ein großer Unterschied zwischen den Positionen 560 und 570 besteht nun hinsichtlich der Abschreibungs- und Zinsaufwendungen. Finden sich derartige Aufwandsposten im Abschnitt 560 recht selten, so machen doch mittlerweile einige Kommunen innerhalb des Abschnitts Badeanstalten davon Gebrauch. ${ }^{43}$

Auf der Einnahmeseite wird grundsätzlich unterschieden zwischen den Eintrittsgeldern, möglichen Pachten für Verkaufsstände, der eingenommenen Umsatz- und Vorsteuer, möglichen Betriebskostenerstattungen, Entgelten aus Automaten, sonstigen Verkaufserlösen und zum Teil einigen weiteren Positionen.

\section{b) Der Vermögenshaushalt}

Im Vermögenshaushalt unterscheidet sich dieser Posten kaum vom Abschnitt Sportstätten. Auf der Ausgabeseite werden sämtliche investive Maßnahmen im Bereich der kommunalen Badeanstalten erfaßt. Einnahmen gibt es innerhalb dieser Position vorrangig in Form von Zuweisungen der Bundesländer oder privater Unternehmungen zu verbuchen.

\footnotetext{
${ }^{43}$ Vgl. Haushaltspläne der Stadt Wolfsburg. Die Gemeinde Wolfsburg bspw. weist im Unterabschnitt $570 \mathrm{ab}$ dem Haushaltsplan 1974 explizit die Abschreibungsaufwendungen und Fremdkapitalzinsen für die kommunalen Badeanstalten aus, in den Jahren davor verwandte sie eine Position Ausgaben der Vermögensbildung. Ähnliches zeigte ein Blick in den Haushalt von Cuxhaven; vgl. Haushaltspläne der Stadt Cuxhaven. Auch die Stadt Mönchengladbach führt die Bäder seit dem 01.01.1989 als Betriebe gewerblicher Art und kompensiert den kameralen Überschuß durch die in der kaufmännischen Buchführung nachgewiesenen Abschreibungen und Zinsen, wenngleich ein expliziter Ausweis der Abschreibungs- und Zinsaufwendungen innerhalb des Abschnitts 570 nicht erfolgt. Vgl. Haushaltsplan 1990 der Stadt Mőnchengladbach, S. 309.
} 


\subsubsection{Sportförderung außerhalb des Einzelplanes 5}

Ein Aspekt, der im Zusammenhang mit der Betrachtung kommunaler Sportetats zunehmende Bedeutung erlangt, ist die Ausgliederung diverser Sportausgaben aus dem Einzelplan 5. Die oben erwähnte Abfrage der Sportausgaben von über 500 Gemeinden im Haushaltsjahr 1991 zeigte, daß knapp 15\% der betrachteten Kommunen die Ausgaben im Zusammenhang mit Badeanstalten nicht oder nicht vollständig innerhalb des Haushaltsabschnitts 570 Badeanstalten ausweisen. ${ }^{44}$ Diese Kommunen führen die Badeanstalten zumeist als Eigenbetrieb in Form von Gesellschaften mit beschränkter Haftung im Rahmen der Stadtwerke. Innerhalb des Abschnitts 570 erfolgt dann entweder gar kein Ausweis, ${ }^{45}$ oder aber es werden nur die jeweiligen Betriebskostenzuschüsse, ${ }^{46}$ evtl. auch Abschreibungen und Verzinsungen des Anlagekapitals ausgewiesen, ${ }^{47}$ was letztlich zu einem geringeren Ausweis an Sportausgaben führt und den Vergleich zwischen den jeweiligen Haushaltsabschnitten verschiedener Kommunen erschwert. 48

Ein ähnliches Bild zeigte sich hinsichtlich einzelner Sportstätten, die auch dem Schulsport dienen und von verschiedenen Kommunen im Schuletat (Einzelplan 2) ausgewiesen werden. 49

Neben den Badeanstalten, bei denen eine Auslagerung aus dem städtischen Etat und die Verwaltung durch eine $\mathrm{GmbH}$ relativ häufig anzutreffen ist, gibt es auch einige wenige Kommunen, die bestimmte Sportstätten ausgelagert haben und als $\mathrm{GmbH}$ führen. Ein sehr bekanntes Beispiel hierfür stellt der Olympiapark in München dar. ${ }^{50}$

Abschließend soll folgendes festgehalten werden. Die betrachteten Kommunen unterscheiden sich mitunter hinsichtlich ihrer haushaltstechnischen Vorgehensweise. Ein Teil der Kommunen weist kalkulatorische Kosten der Überlassung von Sportstätten, kalkulatorische Abschreibungen oder Zinsen aus. Dies führt c.p. zu unterschiedlichen Sportbudgets mit der Folge, daß aussagekräftige interkommunale Vergleiche erschwert werden.

${ }^{44} \mathrm{Vgl}$. Hockenjos, 1992, S. 278 - 305.

${ }^{45}$ Vgl. u.a. die Haushalte der Städte Ahlen, Biberach an der Riß, Bocholt, Elmshorn, Frechen, Gaggenau, Husum, Ingolstadt, Kempen, Offenburg, Reutlingen, Rottenburg a.N., Rottweil und Worms.

${ }^{46} \mathrm{Vgl}$. u.a. die Haushalte der Städte Darmstadt, Hattingen, Hofheim am Taunus, München und Regensburg.

${ }^{47} \mathrm{Vgl}$. Haushaltspläne der Stadt Herborn.

${ }^{48} \mathrm{Am}$ Zuschußbedarf ändert sich dadurch letztlich nichts, die Bruttosumme des Sportetats wird jedoch auf diese Weise gekürzt.

${ }^{49}$ So ergänzte bspw. die Stadt Mönchengladbach, daß die Unterhaltungs- und Bewirtschaftungskosten für insgesamt 44 Turn- und 11 Sporthallen nicht erfaßt werden können, da es sich um sogenannte Schultumhallen handelt, die im Schuletat in Verbindung mit den Schulgebäuden erfaßt und bewirtschaftet würden. Gleichwohl stünden diese Hallen aber täglich ab $17 \mathrm{Uhr}$ und an Wochenenden dem Vereinssport zur Verfügung. Ähnliches wurde u.a. auch von den Städten Herdecke und Speyer angefügt.

${ }^{50} \mathrm{Vgl}$. Haushaltspläne der Stadt München. 


\subsubsection{Sportstättenleitpläne als Instrument kommunaler Sportentwicklungs- planung}

Die Idee einer gemeindlichen Sportstättenentwicklungsplanung als integrativem Bestandteil einer weitsichtigen Sport- und auch der gesamten kommunalen Entwicklungsplanung ist nicht neu und geht in ihren Ansätzen bereits auf die Entwürfe eines Reichsspielplatzgesetzes von 1912 und 1920 zurück. ${ }^{51}$

Systematisiert und konkretisiert wurden derartige Überlegungen wesentlich später, ab Mitte der 50er Jahre, auf Initiative des Deutschen Sportbundes, der Deutschen Olympischen Gesellschaft, des Deutschen Städtetages, des Deutschen Städte- und Gemeindebundes sowie des Bundesinstituts für Sportwissenschaft, die zum Entstehen einiger, heute noch gültiger Orientierungshilfen für die kommunale Sportstättenplanung beigetragen haben.

Es sind dies insbesondere die weiter oben genannten DOG-Richtlinien, ${ }^{52}$ die Orientierungshilfen für die Planung und Errichtung für spezielle Anlagen des Deutschen Sportbundes ${ }^{53}$ sowie die Richtmaße des Bundesinstituts für Sportwissenschaft. ${ }^{54}$

Die sportartenunabhängigen DOG-Richtlinien beinhalten konkrete Angaben zum Bedarf an Kernsportstätten und definieren den jeweiligen örtlichen Bedarf in Abhängigkeit von der Einwohnerzahl. Dieser wird für Sportplätze grundsätzlich mit 4 und für Sporthallen mit 0,2 qm sportlich nutzbarer Fläche pro Einwohner beziffert. Weitere Kriterien sind die Richtmaße für einzelne Anlagen, Standort, Entfernung etc.

Die 1984 gemeinsam vom Deutschem Sportbund, den kommunalen Spitzenverbänden und dem Deutschem Landkreistag erarbeiteten Orientierungshilfen greifen Sportstätten für spezielle Sportarten, d.h. vorrangig Sondersportanlagen auf. Im Gegensatz zu den DOG-Richtlinien werden hierbei die Bedarfswerte nicht in Abhängigkeit von der Einwohnerzahl, sondern von der Zahl der in der jeweiligen Sportart Aktiven bestimmt, womit insbesondere örtlichen Gegebenheiten, Traditionen u.ä. verstärkt Rechnung getragen werden soll.

Die Richtmaße des Bundesinstituts für Sportwissenschaft geben keinerlei städtebauliche Orientierungswerte in Form von qm pro Einwohner oder Aktiven vor, sondern konkretisieren die Objektplanung durch Ausführungen zu Anlagenmaß und -größe.

\footnotetext{
${ }^{51}$ Vgl. Pelshenke, 1961, S. 5.

S2Vgl. Deutsche Olympische Gesellschaft, 1956.

53Vgl. Deutscher Sportbund, 1984, "Orientierungshilfen für die Planung und Errichtung von Speziellen Anlagen für einzelne Sportarten", Ergänzung des Kapitels V der "Richtlinien für die Schaffung von Erholungs-, Spiel- und Sportanlagen", III. Fassung, 1976, Frankfurt a.M., 1984.

${ }^{54} \mathrm{Vgl}$. Bundesinstitut für Sportwissenschaft, 1984, "Spezielle Anlagen für einzelne Sportarten - Richtmaße", Köln, 1984.
} 
Über die Bewertung derartiger Vorgaben herrscht geteilte Meinung. Wenngleich auch von der Deutschen Olympischen Gesellschaft selbst angemerkt wird, daß es sich dabei lediglich um Orientierungshilfen handelt, 55 stehen die kommunalen Spitzenverbände den oben genannten DOG-Richtlinien und ihren klar definierten Bedarfswerten mitunter skeptisch gegenüber. Es sei, so bspw. der Deutsche Städte- und Gemeindebund, eine Berücksichtigung regionaler und vor allem lokaler Gesichtspunkte für die gemeindliche Entwicklungsplanung unverzichtbar, wie es auch in den "Richtwerten" deutlich zum Ausdruck kommt. Eine in die gleiche Richtung zielende Stellungnahme wird deutlicher und verlangt:

"Was wir also benötigen, ist nicht ein zweiter Goldener Plan; es sind vielmehr 8500 kommunale Goldene Planungen für die sportpolitische Weiterentwicklung unserer Städte und Gemeinden." 56

Nichtsdestotrotz haben vor allem die DOG-Richtlinien Mitte der 70er Jahre zu einer Fülle von Planungen geführt, über deren Realisierung jedoch weitestgehend Unsicherheit besteht. ${ }^{57}$

Haass kommt in einer Studie zur kommunalen Sportentwicklungsplanung zum Ergebnis, daß sich 45 der 50 von ihm untersuchten Kommunen bezüglich der Aufstellung von Sportstättenleitplänen an den Bedarfsrichtwerten der Deutschen Olympischen Gesellschaft orientierten. ${ }^{58}$ Zwar stellt Haass ebenfalls fest, daß einige Kommunen Kritik an den DOG-Richtwerten äußerten, dennoch hätten sich nur wenige Kommunen explizit von den Richtwerten abgewandt und eigene Bedarfswerte angesetzt.

Nun führt die Aufstellung eines Planes bekanntlich nicht zwangsläufig zu dessen Realisierung, was anhand folgender Zahlen kurz illustriert werden soll. Betrachtet man den Sportstättenbestand in den gut 500 bundesdeutschen Kommunen mit mehr als 20.000 Einwohnern, dann zeigt sich folgendes Bild:59

Der durchschnittliche Bestand an Sportplatzflächen betrug im Jahr 1990 etwa 3,25 $\mathrm{qm} /$ Einwohner, jener an Sporthallenflächen etwa $0,22 \mathrm{qm} /$ Einwohner. Die reine Durchschnittsbetrachtung kann folglich bestätigen, daß die oben genannten Richtwerte

\footnotetext{
${ }^{55} \mathrm{Vgl}$. Deutsche Olympische Gesellschaft, 1956, S. 1. Auch wird im Vorwort auf die Zustimmung seitens der Kommunalen Spitzenverbände mit verschiedenen Maßgaben verwiesen.

${ }^{56}$ Mombaur/ Mattner-Stellmann, 1988, S. 13. Ein zweiter Goldener Plan zum gegebenen Zeitpunkt wurde von der Deutschen Olympischen Gesellschaft bereits 1960, im zweiten Memorandum zum Goldenen Plan angekündigt.

${ }^{57} \mathrm{Vgl}$. Mattner, 1984, S. $617 \mathrm{f}$.

${ }^{58} \mathrm{Vgl}$. Haass, 1990, S. $24 \mathrm{ff}$.

${ }^{59}$ Die Ausfuihrungen basieren auf einer Erhebung von Duss bezüglich des Sportstättenbestands in der Bundesrepublik Deutschland im Jahr 1990, abgedruckt im Statistischen Jahrbuch Deutscher Gemeinden. Vgl. Duss, 1990, S. $228 \mathrm{ff}$.
} 
in Höhe von 4 bzw. 0,2 qm/Einwohner tatsächlich größtenteils realisiert wurden, was insbesondere im Hinblick auf die Sporthallen festzustellen ist.

Berücksichtigt man jedoch auch die Streuung dieser durchaus repräsentativen Stichprobe, dann lassen sich gleichwohl andere Schlüsse ziehen. Die Standardabweichung beträgt bei den Sportplätzen 1,66, der resultierende Variationskoeffizient beläuft sich auf 0,51. Im Zusammenhang mit Sporthallen beträgt die Standardabweichung 0,07 und der Variationskoeffizient liegt bei 0,3 .

Damit kann festgehalten werden, daß sich die Kommunen bezüglich des Bestandes an Sportstätten, insbesondere an Sportplätzen, beträchtlich unterscheiden. Von einer Nivellierung kommunaler Sportstättenpolitik aufgrund der oben genannten Orientierungshilfen kann kaum die Rede sein. 


\subsubsection{Qualitative Aspekte kommunaler Sportförderung - eine Analyse kom- munaler Sportförderrichtlinien und -praxis}

\subsubsection{Die Empfehlungen des Deutschen Städtetages als Grundlage kom- munaler Sportförderrichtlinien}

Wie bereits erwähnt, sind es insbesondere größere Kommunen, ${ }^{60}$ die sich bezüglich der Detailgestaltung der Sportförderung sogenannter Sportförderrichtlinien bedienen. Die vom Deutschen Städtetag in diesem Zusammenhang erarbeiteten Empfehlungen zur kommunalen Sportförderung sollen den Gemeinden die Formulierung derartiger Richtlinien erleichtern und zeichnen sich des weiteren durch folgende Zielsetzung aus: Sportförderrichtlinien sollen dazu dienen, mehr Transparenz in der Sportpolitik zu schaffen. Daneben soll etwas mehr Vergleichbarkeit und Vereinheitlichung der kommunalen Sportförderpraxis erreicht werden, obschon die Unabhängigkeit der Kommunen und die Abhängigkeiten der Sportpolitik von den örtlichen Gegebenheiten sowie der Finanzkraft einer Stadt hervorgehoben wird. ${ }^{61}$

Im einzelnen geht der Städtetag auf die Unterscheidung zwischen organisiertem und nichtorganisiertem Sport ein und erwähnt, daß Sportförderung grundsätzlich die gesamte Bevölkerung innerhalb und außerhalb der Vereine erfasse. Förderungswürdig seien demnach neben dem Vereinssport auch eigene städtische Sportangebote bspw. mit dem Ziel einer Hinführung zu regelmäßiger Sportausübung in den Vereinen und weitere, nicht näher definierte Formen des nichtorganisierten Sports, bei denen gesundheitliche, freizeitorientierte und gesellige Motive im Vordergrund stünden. Kommerzielle Sportangebote bezeichnet der Städtetag als förderungswürdig im Sinne einer Ergänzung, beschränkt den Bereich der Unterstützung dieser Form des Sportangebots jedoch auf allgemeine Wirtschaftsförderung wie Ausweisung und Verkauf oder Verpachtung von Grundstücken sowie Unterstützung bei Bauanträgen. ${ }^{62}$

Im weiteren Verlauf werden konkret einzelne Fördergegenstände angesprochen.

So sprechen sich die Empfehlungen hinsichtlich der Errichtung von Sportstätten für die Kommunen als Bauherren aus und räumen dabei den sogenannten Kernsportstätten Vorrang vor Sondersportanlagen ein. In diesem Zusammenhang weisen sie auf die Nützlichkeit von Sportstättenentwicklungsplänen hin, um eine möglichst sinnvolle Stadtentwicklung zu gewährleisten. ${ }^{63}$

\footnotetext{
${ }^{60}$ Mit dem Begriff "Größere Konumunen" in diesem Sinne sind die Gemeinden in einer Größenordnung von mehr als 20.000 Einwohnern angesprochen.

${ }^{61}$ Vgl. Deutscher Städtetag, 1987, S. 3.

${ }^{62}$ Vgl. Deutscher Städtetag, 1987, S. 4 f.

${ }^{63}$ Vgl. Deutscher Städtetag, 1987, S. 6. Zu den Sportstättenentwicklungsplänen, vgl. Haass, 1990. 
Hinsichtlich der Frage nach den Nutzungsregelungen für kommunale Sportstätten sprechen sich die Empfehlungen des Städtetages in Abhängigkeit von der jeweiligen Sportanlage mitunter für eine Übertragung der Schlüsselverantwortung an die Vereine aus. 64

Wo eine derartige Verantwortungsübergabe nicht sinnvoll erscheint, lehnt der Städtetag Benutzungsentgelte für den allgemeinen Übungs- $u$. Trainingsbetrieb der Vereine und - in etwas abgeschwächter Form - auch für die Nutzung durch den vereinsungebundenen Sport grundsätzlich ab. Relativiert wird die Aussage bezüglich der grundsätzlich kostenlosen Bereitstellung allerdings insofern, als für eine angemessene Kostenbeteiligung bei besonders kostenintensiven Einrichtungen wie Bädern, Veranstaltungshallen oder Stadien geworben wird. ${ }^{65}$

Für Vereine mit eigenen Sportstätten empfiehlt der Deutsche Städtetag aufgrund deren stärkerer Kostenbelastung eine kommunale Unterstützung sowohl bei den Investitionen als auch bei den folgenden Unterhaltungskosten. ${ }^{66}$

Im Hinblick auf den Berufssport vertritt der Deutsche Städtetag die Ansicht, daß selbiger aus dem kommunalen Sportetat nicht gefördert werden sollte. Eine Unterstützung dieses Sportbereichs sei aus anderen Gesichtspunkten, wie z.B. Wirtschaftsförderung und Öffentlichkeitsarbeit einer Stadt möglich. Kommunale Sportstätten, so die Empfehlungen weiter, werden dem Berufssport in der Regel nur gegen Entgelt überlassen.67

Schließlich nennen die Empfehlungen einige weitere Förderregelungen und gehen dabei auf die Förderung einzelner Aktivitäten ein, die allerdings, noch viel stärker als der Bau und die Unterhaltung von Kernsportstätten, von den örtlichen Gegebenheiten, der Finanzkraft der Stadt u.ä. abhängig seien.

Hierzu gehören im einzelnen Reisekostenzuschüsse für die Teilnahme an Spitzensportveranstaltungen, die Förderung solcher Veranstaltungen sowie Unterstützungen zur Anschaffung von Sportgeräten. Die Gewährung von Übungsleiterzuschüssen, eine spezielle Förderung bestimmter Personengruppen wie bspw. die Jugendarbeit oder die

\footnotetext{
${ }^{64}$ Eine Übertragung der Schlüsselverantwortung meint die Übergabe von Sportstätten von den Kommunen an die Vereine. Ziel solcher Übergaben ist es u.a., daß die Vereine zumindest teilweise für die Unterhaltung der ihnen übertragenen Sportplätze Sorge tragen mögen. Die Trägerschaftsverhältnisse nordrhein-westfälischer Sportstätten konnten zeigen, daß von diesem Instrument vorrangig im Zusammenhang mit Sportplätzen durchaus Gebrauch gemacht wurde. So sank der Anteil der kommunal geführten Sportplätze in diesem bevölkerungsreichsten Bundesland von $89 \%$ im Jahre 1980 innerhalb von nur acht Jahren auf $50 \%$.

${ }^{65}$ Konkretisiert wird der Begriff der Angemessenheit allerdings nicht.

${ }^{66}$ Die exakte Formulienung innerhalb der Empfehlungen lautet: "[...] ist eine kommunale Unterstützung sowohl bei den Investitionen [...] als auch bei den Folgekosten [...] denkbar." Vgl. Deutscher Städtetag, 1987, S. 6.

${ }^{67} \mathrm{Vgl}$. Deutscher Städtetag, 1987, S. 8.
} 
Förderung des Versehrten- und Seniorensports und einiges mehr vervollständigen das breite Spektrum der Vorschläge.

Es soll an dieser Stelle darauf hingewiesen werden, daß die weiter oben erwähnte Kritik der kommunalen Spitzenverbände respektive des Deutschen Städte- und Gemeindebundes an den DOG-Richtlinien als einem "Imperativ"68 nicht ganz nachvollzogen werden kann, wenn man die ebenfalls recht konkreten Vorschläge des Deutschen Städtetages für die Ausgestaltung kommunaler Sportförderrichtlinien berücksichtigt. Einschränkend soll jedoch abermals darauf hingewiesen werden, daß sich beide sportpolitischen Empfehlungen lediglich als Vorschläge, keineswegs aber als strikte Vorgaben verstehen.

Im folgenden werden die Inhalte derartiger Sportförderrichtlinien etwas näher betrachtet. Dabei werden mögliche Parallelen oder aber Unterschiede innerhalb der untersuchten Richtlinien bzw. der kommunalen Sportförderpraxis analysiert, um letztlich allgemeine Aussagen über qualitative Aspekte kommunaler Sportförderung ableiten zu können. ${ }^{69}$

$\mathrm{Zu}$ diesem Zweck wurden die Richtlinien von 70 Kommunen verschiedener Größenordnung und geographischer Lage fernmündlich angefordert, die Sportämter der in die Betrachtung eingehenden baden-württembergischen Kommunen wurden größtenteils persönlich aufgesucht. ${ }^{70}$ Es zeigte sich, daß fast alle Kommunen in einer Größenordnung von mehr als 20.000 Einwohnern Sportförderrichtlinien besitzen; lediglich in fünf Fällen war vom zuständigen Sportamt zu erfahren, daß von diesem Instrument kommunaler Sportpolitik kein Gebrauch gemacht werde. ${ }^{71}$ Letztlich konnten 61 Kommunen in die Betrachtung eingehen. ${ }^{72}$

${ }^{68} \mathrm{Vgl}$. Mattner, 1984, S. 621.

${ }^{69}$ Ergänzend zu dieser Analyse wird auf Erhebungen seitens der Städte Braunschweig, Esslingen am Neckar und Karlsruhe zurückgegriffen .

${ }^{70}$ Die persönlichen Interviews mit baden-württembergischen Sportämtern wurden vorrangig im Jahr 1993 durchgeführt, die Ausdehnung der Untersuchung über die baden-württembergische Grenzen hinweg erfolgte im Jahr 1994.

${ }^{71}$ Von den interviewten Kommunen mit mehr als 20.000 Einwohnern gaben lediglich Baden-Baden, Buxtehude, Cuxhaven und Sindelfingen an, keine Sportförderrichtlinien zu besitzen. Das Sindelfinger Sportamt verwies darauf, daß dieses Instrument aus Gründen der Finanzknappheit abgeschafft wurde. Kleinere Kommunen hingegen verzichten oftmals auf Sportförderrichtlinien und regeln die Fragen des Sports ohne explizit schriftlich verfaßte Grundsätze und dergleichen.

${ }^{72} \mathrm{Im}$ einzelnen handelt es sich dabei um die Städte Aachen, Augsburg, Bad Salzuflen, Bochum, Bruchsal, Delbrück, Dortmund, Dreieich, Forchheim, Freiburg, Fröndenberg, Ganderkesee, Geislingen an der Steige, Heidelberg, Heilbronn, Hof, Homburg, Karlsruhe, Kehl, Koblenz, Konstanz, Köln, Lahr, Langen, Lehrte, Leimen, Lennestadt, Lörrach, Leverkusen, Limburg, Lübeck, Lüdenscheid, Mannheim, München, Neumünster, Neunkirchen, Neustadt am Rübenberge, Neu-Ulm, Nürnberg, Offenburg, Osnabrück, Passau, Pforzheim, Pinneberg, Rastatt, Rendsburg, Reutlingen, Saarbrücken, St. Augustin, Singen, Solingen, Stuttgart, Tübingen, Ulm, Villingen-Schwenningen, Wetter, Wetzlar, Wolfsburg, Worms, Würselen und Würzburg. 
Die Betrachtung des Aufbaus der Sportförderrichtlinien ergab folgendes Bild:

Die verschiedenen Sportförderrichtlinien differieren bezüglich ihres gliederungstechnischen Aufbaus erheblich. Ähnliches konnte hinsichtlich des Umfangs festgestellt werden, wobei hier eine tendenzielle Abhängigkeit von der Gemeindegröße zu beobachten war.

\subsubsection{Allgemeine Voraussetzungen für den Erhalt direkter öffentlicher Zuwendungen}

Der Großteil der betrachteten Kommunen nennt als Antragsberechtigte die gemeinnützigen Sportvereine mit Sitz in der jeweiligen Stadt, erwartet zumeist auch eine Eintragung ins Vereinsregister und verlangt die Mitgliedschaft in einem dem DSB zugehörigen Verband, womit eine direkte Fördenung alternativer Sportanbieter von vornherein explizit ausgeschlossen wird.

83,6\% der untersuchten Kommunen stellen die Notwendigkeit der Antragstellung zum Erhalt der öffentlichen Gelder explizit dar und eine ähnlich hohe Anzahl fordert auch einen Verwendungsnachweis für die verausgabten Gelder. Die Antragserfordernis zeichnet sich in aller Regel dadurch aus, daß der antragstellende Verein selbigen bis zu einem bestimmten Zeitpunkt beim zuständigen Amt, in aller Regel dem städtischen Sportamt oder -dezernat, einreichen muß. Als Bestandteile dieses Antrags, insbesondere bei Bauvorhaben, werden von etlichen Gemeinden Baugenehmigungen, Kostenvoranschläge, Folgelastenberechnungen, Finanzierungsnachweise bzw. -pläne, u.ä. erwartet.

In 40 von 61 Fällen wird das Vorhandensein von Eigenmitteln explizit als unabdingbare Voraussetzung für die Gewährung von Geldern genannt, wodurch diese Kommunen dem Grundsatz der Subsidiarität Rechnung tragen wollen. Offen lassen die Kommunen hingegen, wie hoch dieser Anteil im einzelnen sein sollte. So werden in den meisten Fällen lediglich Eigenmittel in angemessener Höhe erwartet, die zum Teil auch durch Eigenarbeit erbracht werden können. ${ }^{73}$

\footnotetext{
${ }^{73}$ Der Badische Sportbund empfiehlt diesbezüglich die Anerkennung von bis zu $50 \%$ der zuschußfähigen Kosten in Höhe von 20,- DM je Arbeits- oder Maschinenstunde. Vgl. Badischer Sportbund, 1992. 
Stadler verweist hinsichtlich des vereinseigenen Sportstättenbaus auf die große Bedeutung der Subsidiarität und faßt die Verteilung der Finanzierungslasten in diesem Bereich wie folgt zusammen: 74

- ca. $10-30 \% \quad$ kommunale Zuschüsse

- ca. $30 \% \quad$ Zuschüsse vom Land/Sportbund

- ca. $10 \% \quad$ sonstige Zuschüsse

- ca. $40 \% \quad$ Eigenarbeit/ Eigenmittel/ Kapitalaufnahme

De facto bedeutet dies, daß mindestens die Hälfte der Kosten für den Bau von vereinseigenen Sportstätten von den öffentlichen Haushalten getragen werden.

Etwas mehr als die Hälfte der Kommunen stellt des weiteren Bedingungen an die Höhe der Mitgliedsbeiträge. Die meisten bleiben jedoch, ähnlich wie bei der Erfordernis von Eigenmitteln, unbestimmt und verlangen lediglich Angemessenheit. ${ }^{75} 12$ Kommunen hingegen nennen konkret Mindestbeträge und bewegen sich dabei zwischen 2,- $\mathrm{DM}^{76}$ und 12,- DM77 Monatsbeitrag für erwachsene Mitglieder.

Tabelle 5: Mitgliedsbeiträge als Voraussetzung der Zuschußgewährung

\begin{tabular}{|l|l|l|}
\hline Anforderungen an die Mitgliedsbeiträge & Anzahl der Kommunen & in \% \\
\hline Keine Nennung & 24 & 39,3 \\
\hline Angemessenheit der Mitgliedsbeiträge & 25 & 41 \\
\hline Explizite Nennung der Mindestbeitragshöhe & 12 & 19,7 \\
\hline
\end{tabular}

Die Mitgliedsbeiträge für Jugendliche (unter 18 Jahren) betragen, soweit aufgeführt, etwa die Hälfte der erwarteten Erwachsenenbeiträge. In bezug auf die Struktur der Mitgliedschaft nennen einige wenige Kommunen eine Mindestmitgliederzahl (50 bis 150) oder verlangen, daß ein bestimmter Mindestprozentsatz der Mitglieder (meist $50 \%$ ) Bürger der betreffenden Kommune sind.

${ }^{74} \mathrm{Vgl}$. Stadler, 1993.

${ }^{75}$ Zur Angemessenheit der Mitgliedsbeiträge: Der Landessportbund Nordrhein-Westfalen bezeichnet Beiträge von 3,- DM monatlich für Kinder bis 14 Jahre, 4,- monatlich für Jugendliche bis 18 Jahre und 5,- monatlich für Erwachsene als angemessen. Vgl. LSB Nordrhein-Westfalen, 1991.

${ }^{76} \mathrm{Vgl}$. Stadt Reutlingen, 1988.

77 Vl. Stadt Stuttgart, 1993. 
Tabelle 6: Allgemeine Voraussetzungen für den Erhalt direkter öffentlicher Zuwendungen

\begin{tabular}{|l|l|l|}
\hline Kriterium & Anzahl der Kommunen & in \% \\
\hline Sportvereine & 58 & 95,1 \\
\hline Gemeinnützigkeit & 31 & 50,1 \\
\hline Sitz in der jeweiligen Stadt & 51 & 83,6 \\
\hline Eintragung ins Vereinsregister & 16 & 26,2 \\
\hline Mitgliedschaft im DSB o.ä. Verband & 49 & 80,3 \\
\hline Antragserfordernis & 51 & 83,6 \\
\hline Erfordernis eines Verwendungsnachweises & 49 & 80,3 \\
\hline Erfordernis von Eigenmitteln & 40 & 65,6 \\
\hline $\begin{array}{l}\text { Hinweis auf das Nichtvorhandensein von Rechtsan- } \\
\text { sprüchen bzw. Abhängigkeit der Mittelvergabe von der } \\
\text { finanziellen Lage }\end{array}$ & 49 & 80,3 \\
\hline
\end{tabular}

Nicht zuletzt verweisen etliche der untersuchten Gemeinden auf die notwendige Bereitschaft einer Zurverfügungstellung der jeweiligen Sportstätten für den Schulsport oder auch sonstige städtische Aktivitäten.

$80 \%$ der Kommunen stellen schließlich ausdrücklich dar, daß bezüglich der Vergabe von Fördermitteln keinerlei Rechtsansprüche bestehen bzw. daß sich die Höhe der Sportfördermittel nach der jeweiligen Haushaltslage richtet.

Als Ergebnis soll festgehalten werden:

Die Untersuchung der allgemeinen Voraussetzungen belegt, daß öffentliche Sportförderung im Sinne direkter Zuwendungen (Haushaltsabschnitt 550) eine reine Vereinsförderung ist und zeigt darüber hinaus, daß den Sportförderrichtlinien als Instrument zur Schaffung von Transparenz kommunaler Sportpolitik Grenzen gesetzt sind, läßt sich doch der Begriff der Angemessenheit der Mitgliedsbeiträge oder der erforderlichen Eigenmittel kaum greifen.

Daneben wird mit der Betonung auf das Nichtvorhandensein von Rechtsansprüchen ein erster Hinweis auf einen möglichen Bestimmungsfaktor kommunaler Sportförderung gegeben. 


\subsubsection{Zuschüsse zu BaumaBnahmen der Vereine}

Alle untersuchten Sportförderrichtlinien beinhalten Regelungen zur Gewährung von Zuschüssen für den Bau oder die Sanierung vereinseigener Sportanlagen.

Weitaus uneinheitlicher präsentiert sich hingegen die Detailgestaltung zur Vergabe von Investitionszuschüssen. 10 Gemeinden nennen als Zuschußhöhe einen bestimmten Prozentsatz der Investitionskosten, wobei dieser entweder als grundsätzlicher Wert ${ }^{78}$ oder - in den meisten Fällen - als Maximalwert definiert ist. Die Maximalwerte reichen von 20 bis hin zu 50\%. ${ }^{79}$

Ein wesentlich größerer Teil der Kommunen bedient sich zwar ebenfalls eines bestimmten Maximalprozentsatzes als Richtwert, bezieht diesen dann allerdings nicht auf die tatsächlichen Baukosten, sondern auf die zuschußfähigen oder anerkannten anrechnungsfähigen Kosten.

Nun sind diese anrechnungsfähigen Kosten wiederum nicht Bestandteil der Förderrichtlinien und auch keineswegs in allen Kommunen identisch. ${ }^{80}$ Die Kommunen orientieren sich zwar grundsätzlich an den diesbezüglichen Richtlinien der jeweiligen Landessportbünde, die in ihren Richtlinien zum vereinseigenen Sportstättenbau konkrete Angaben zum zuschußfähigen Höchstaufwand bei verschiedenen Baumaßnahmen machen, sie sind jedoch keineswegs verpflichtet, diesen Vorgaben strikt zu folgen ${ }^{81}$

Sportartenspezifische Unterscheidungen werden interessanterweise von recht wenigen Kommunen getroffen. ${ }^{82}$ Wo dies der Fall ist, kann eine leichte Tendenz hin zu vergleichsweise höheren Fördersätzen von Kernsportstätten beobachtet werden. ${ }^{83}$ Es erscheint jedoch fraglich, daraus eine grundsätzlich höhere Förderung von Kernsportstätten interpretieren zu wollen. Selbst wenn eine Kommune für eine bestimmte Sportart niedrigere Fördersätze angibt, ist damit noch längst nicht belegt, daß diese Sportart tatsächlich weniger gefördert wird. Die Angabe von Maximalsätzen wie auch das Nichtvorhandensein von Rechtsansprüchen lassen den kommunalpolitischen Entscheidungsträgern weiterhin genügend Spielraum, der von Außenstehenden kaum nachvollzogen werden kann.

\footnotetext{
${ }^{78} \mathrm{Vgl}$. hierzu u.a. die Sportförderrichtlinien der Städte Offenburg und Passau, die als grundsätzliche Zuschußhöhe $10 \%$ der anfallenden Investitions- oder Baukosten ausweisen.

${ }^{79} \mathrm{Vgl}$. zu letzterem u.a. die Sportförderrichtlinien der Städte Ulm und Wolfsburg.

${ }^{80}$ Letztere Information konnte durch ein Gespräch mit dem Sportamt der Stadt Karlsruhe gewonnen werden.

${ }^{81}$ Vgl. hierzu Badischer Sportbund, 1992. So beträgt der zuschußfähige Höchstaufwand auszugsweise für Spielfelder DM 450.000, für Turn- und Gymnastikhallen DM 300.000, für Tennisplätze DM 60.000 und für Tennishallen DM 880.000 .

${ }^{82}$ Es waren dies vorrangig die größeren baden-württembergischen Kommunen.

${ }^{80}$ Vgl. u.a. die Sportförderrichtlinien der Städte Heilbronn, Mannheim, Pforzheim, Ulm.
} 
Tabelle 7: Fördersätze für den Bau vereinseigener Sportanlagen

\begin{tabular}{|c|c|c|c|c|}
\hline \multicolumn{2}{|c|}{ Fördersätze } & Anzahl der Kommunen & in $\%$ & \\
\hline \multicolumn{2}{|c|}{ keine Zuschüsse } & 0 & 0 & \\
\hline \multicolumn{2}{|c|}{ Zuschüsse } & 61 & 100 & \\
\hline \multirow[t]{12}{*}{ darunter: } & $\begin{array}{l}\text { als genereller \%-satz der Investitions- } \\
\text { oder Bau-Kosten }\end{array}$ & 10 & 16,4 & 100 \\
\hline & $0-10 \%$ & 4 & & 40 \\
\hline & $11-20 \%$ & 4 & & 40 \\
\hline & $21-30 \%$ & 0 & & 0 \\
\hline & $>30 \%$ & 2 & & 20 \\
\hline & $\begin{array}{l}\text { als genereller \%-satz der anrechnungs- } \\
\text { fähigen Investitions- oder Bau-Kosten }\end{array}$ & 32 & 52,5 & 100 \\
\hline & $0-10 \%$ & 2 & & 6,25 \\
\hline & $11-20 \%$ & 6 & & 18,8 \\
\hline & $21-30 \%$ & 13 & & 40,6 \\
\hline & $>30 \%$ & 11 & & 34,4 \\
\hline & $\begin{array}{l}\text { Unterschiedliche Sätze für verschiedene } \\
\text { Sportanlagen }\end{array}$ & 7 & 11,5 & \\
\hline & keine explizite Nennung der Fördersätze & 12 & 19,7 & \\
\hline
\end{tabular}

Weitere Unterschiede zwischen der Förderpraxis in den verschiedenen Kommunen ergeben sich mitunter durch die Abhängigkeit der Fördersätze von der Frage schulischer Mitbenutzung, der Altersstruktur der Vereinsmitgliedschaft, der Höhe der Zuschüsse von dritter Seite und auch der grundsätzlichen Unterscheidung zwischen Neubau und Sanierungsmaßnahmen.

Zusammenfassend kann festgehalten werden, daß die verschiedenen relevanten Kriterien, der Ausweis von Maximalsätzen und der Bezug auf anrechnungsfähige Kosten einen Vergleich der Förderung des Baus vereinseigener Sportanlagen in den verschiedenen Städten wie auch insbesondere einen Vergleich zwischen der Förderpraxis für verschiedene Sportarten in erheblichem Maße erschweren.

Eine vom Schul- und Sportamt Esslingen am Neckar 1988 durchgeführte Umfrage mit insgesamt 76 Städten im gesamten Bundesgebiet ergab zum Vergleich folgendes Bild: 16 Städte (ca. 20\%) gewährten Baukostenzuschüsse in Höhe von $10-20 \%$ der zuschußfähigen Kosten, 39 Städte (ca. 50\%) in Höhe von $20-30 \%$ der zuschußfähigen Kosten, 19 Städte (25\%) Zuschüsse in Höhe von über $30 \%$ der zuschußfähigen Kosten und 2 Städte gewährten keinerlei Zuschüsse. ${ }^{84}$

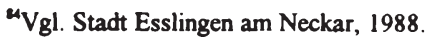




\section{Abbildung 5: Fördersätze für den Bau vereinseigener Sportstätten}

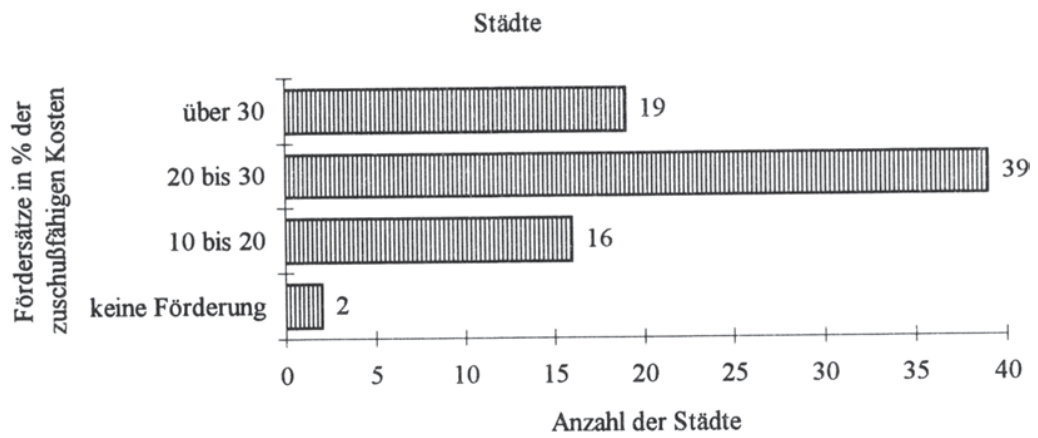

Quelle: Stadt Esslingen, 1990.

Des weiteren ergab dieselbe Studie, daß in lediglich 27 Städten eine Übereinstimmung zwischen den Richtlinien des jeweiligen Landessportbundes und der Förderpraxis existierte. In 49 Städten hingegen, also etwa zwei Drittel der betrachteten Gemeinden, wichen die Zuschußpraktiken mehr oder weniger stark von den Richtlinien des jeweiligen Landessportbundes ab.

Dies unterstreicht zum einen, daß sich die Kommunen ihre Sportpolitik keineswegs gänzlich diktieren lassen. Zum anderen verdeutlicht es abermals die unterschiedliche Handhabung dieses Politikbereichs in verschiedenen Kommunen.

Eine separate Betrachtung verdienen abschließend die weiteren Sportbauten, deren Förderung von 16 der betrachteten Kommunen explizit abgelehnt wird. Als weitere Sportbauten in diesem Sinne gelten Geschäftszimmer, Parkplätze, Tribünen und sonstige Nebenanlagen. Die Förderung des Baus von Vereinsgaststätten wird laut Richtlinien von 22 Kommunen explizit verneint. 


\subsubsection{Kriterien zur Überlassung kommunaler Sportstätten}

Die Untersuchung der Kriterien zur Vergabe kommunaler Sportstätten und der Erhebung von Benutzungsgebühren für selbige erwies sich, ähnlich jener der Investitionszuschüsse, als eminent schwierig, da es sich auch bei der Vergabe- und Entgeltpolitik um einen Bereich kommunaler Sportpolitik handelt, der mitunter sehr differenziert und detailliert geregelt wird. Die Sportförderrichtlinien zeichnen sich in diesem Bereich indirekter Zuwendungen durch eines aus: sie sind völlig uneinheitlich.

Einige Sportförderrichtlinien beinhalten Angaben zur Vergabeordnung oder zu den Gebühren, andere verweisen auf separate Gebührenordnungen. Des weiteren treten Varianten der Preisdifferenzierung auf, die in unterschiedlichem Umfang entweder nach sportstätten-, personen-, zeit- , nutzungszweck-, nutzerbezogenen oder mehreren dieser Kriterien unterscheiden, damit in ein regelrechtes Gebührenwirrwarr münden und einen Vergleich erheblich erschweren.

\section{a) Freiluftsportanlagen}

Kommunale Sportstätten werden oftmals per langfristigem Vertrag an Sportvereine verpachtet. Hierbei handelt es sich zumeist um Sportstätten, die dann in aller Regel auch nur von dem jeweiligen Verein, eventuell auch von Schulen, genutzt werden. Als Beispiele hierfür gelten insbesondere Fußballplätze oder auch bestimmte, nicht vereinseigene Sondersportanlagen wie Tennisplätze und dergleichen.

Die Betrachtung der Benutzungsentgeltordnungen in Zusammenhang mit der langfristigen Verpachtung von Sportstätten zeigte, daß die jeweiligen Vereine mitunter jährliche Pachten und möglicherweise Erbbauzinsen für etwaige Nebenbauten wie Klubhäuser und dergleichen an die Kommune zu entrichten haben.

In den Kommunen, in denen von der Erhebung von Pachten Gebrauch gemacht wird, ${ }^{85}$ bewegen sich selbige im Rahmen von 0,02 bis $0,05 \mathrm{DM} / \mathrm{qm}$ jährlich und haben damit eher symbolischen Charakter. ${ }^{86}$ So bedeutet dies bspw. für einen etwa 7.000 qm groBen Fußballplatz eine jährliche Pacht zwischen 140 bis 350,- DM. Die im Zusammenhang mit Erbbauzinsen angetroffenen Regelungen belaufen sich auf $\pm 4 \%$ auf einen Bodenwert von zumeist um 5,- DM/qm. ${ }^{87}$

\footnotetext{
${ }^{85}$ Vielfach wird auf Pachten völlig verzichtet oder aber die an das Liegenschaftsamt zu entrichtende Pacht wird aus Sportfördermitteln übcrnommen.

${ }^{86}$ Die Pacht für eine Stuttgarter Sportanlage beträgt 0,05 DM/qm jährlich, in Freiburg erhebt die Stadt hierfür 0,02 DM/qm. Ähnliches auch in Pforzheim, wo die Pacht zwar 0,3 DM/qm beträgt, die Vereine davon aber nur $10 \%$ bezahlen müssen. Vgl. Sportförderrichtlinien der Städte Stuttgart, Freiburg und Pforzheim.

${ }^{87}$ So bspw. in der Stadt Freiburg.
} 


\section{b) Sporthallen}

Im Gegensatz zu den bei Freiluftsportanlagen oftmals anzutreffenden langfristigen Pachtverträgen, die de facto einer ausschließlichen Vergabe an einen bestimmten Nutzer gleichkommen, werden insbesondere Sporthallen häufig stunden- oder tageweise vergeben. ${ }^{88}$ Kommunale Sportämter bedienen sich dabei in aller Regel sogenannter Hallenbelegungspläne, welche, jeweils für ein Jahr aufgestellt, die Nutzungszeiten für die verschiedenen Nachfrager regeln. ${ }^{89}$

In Analogie zu dieser stunden- oder tageweisen Vergabe haben die jeweiligen Nutzer vorausgesetzt es wird überhaupt ein Benutzungsentgelt erhoben - dann auch Gebühren pro Stunde, pro Übungseinheit o.ä. zu entrichten. ${ }^{90}$

Ein vorrangig im Zusammenhang mit Benutzungsentgeltregelungen kommunaler Sporthallen anzutreffendes Mittel ist der Gebrauch der verschiedenen, in Abbildung 6 dargestellten Varianten preislicher Differenzierung nach verschiedenen Kriterien.

So werden bspw. Sporthallen in jenen Städten, die zwar grundsätzlich Benutzungsentgelte erheben, den Jugendlichen häufig kostenlos, zumindest jedoch verbilligt zur Verfügung gestellt. ${ }^{91}$ Ebenfalls in Richtung einer jugendorientierten Sportpolitik zielen zeitliche Preisdifferenzierungen nach der jeweiligen Tageszeit. ${ }^{92}$

\footnotetext{
${ }^{88}$ Kurzfristige Vermietungen von Freiluftsportanlagen erfolgen mitunter dann, wenn eine per langfristigem Pachtvertrag an einen bestimmten Verein vermietete Sportanlage gelegentlich auch an weitere Nutzer vergeben wird.

${ }^{89} \mathrm{Als}$ Kriterien zur Vergabe der zumeist knappen Hallenkapazitäten wurde von verschiedenen Sportämtern grundsätzlich die Bevorzugung des Jugend- vor dem Erwachsenensport, bisherige Vergabequoten, die Entwicklung von Vereinsgröße und Anzahl der aktiven Mannschaften wie auch die Frage nach dem generellen Ausübungsort einer Sportart genannt. So wird bspw. einer Hallenhandballmannschaft gegenüber einem Fußballteam generell Priorität eingeräumt.

${ }^{90}$ In einigen Fällen konnte dieser Typus stundenweiser oder ähnlicher Entgelterhebung auch im $\mathrm{Zu}$ sammenhang mit Freiluftsportanlagen, insbesondere Stadien, beobachtet werden. Vgl. u.a. Stadt Leimen, 1992, Anlage 1, Tabelle 1.

${ }^{91}$ Der Sachkostenbeitrag für die Benutzung Stuttgarter Hallen differenziert primär nach alters- und nutzerbezogenen Kriterien. So wendet die Stadt Stuttgart fünf unterschiedliche Kategorien an, um die jeweiligen Preise pro Übungseinheit festzulegen.

Kategorie A, unter $10 \%$ Kinder- und Jugendanteil, DM 7,5/Übungseinheit

Kategorie B, 10 - 23\% Kinder- und Jugendanteil, DM 6,-/Übungseinheit

Kategorie C, 24 - 37\% Kinder- und Jugendanteil, DM 4,-/Übungseinheit

Kategorie D, über 37\% Kinder- und Jugendanteil, DM 3,-/Übungseinheit

Freier Sport, VHS, Betriebssport, DM 15,-/Übungseinheit

Vgl. Stadt Stuttgart, 1993, Anlagen 1-3.

${ }^{92}$ So verlangt bspw. die Stadt Freiburg seit 01.01.1994 eine Gebühr von 15,- DM je Stunde nach 20 Uhr, die sich ab 1995 auf 20,- DM erhöhen soll. Auf eine Erhebung dieser Gebühr vor 20 Uhr wurde aus Rücksichtnahme auf den Jugendsport verzichtet.
} 


\begin{tabular}{|c|c|}
\hline \multirow[t]{3}{*}{ Sportanlagenbezogene P-differenzierung } & Sportstätten \\
\hline & Sporthallen \\
\hline & Badeanstalten \\
\hline Persönliche Preisdifferenzierung & Jugend vs. Erwachsenensport \\
\hline \multirow[t]{2}{*}{ Zeitliche Preisdifferenzierung } & Wochentage vs. Wochenende \\
\hline & Vorabend-vs. Abendstunden \\
\hline Nutzungsbezogene Preisdifferenzierung & $\begin{array}{l}\text { Training u. offizielle Wettkämpfe vs. sonstige } \\
\text { Veranstaltungen }\end{array}$ \\
\hline \multirow[t]{5}{*}{ Nutzerbezogene Preisdifferenzierung } & Sportvereine \\
\hline & sonstige Vereine \\
\hline & freie Gruppen \\
\hline & Betriebssportgruppen \\
\hline & Kommerzielle Nutzer \\
\hline
\end{tabular}

Weitere zeitliche Preisdifferenzierungen erfolgen, allerdings in nur wenigen Städten, nach dem jeweiligen Wochentag. So kostet die Nutzung einer kommunalen Sporthalle am Wochenende in den betreffenden Kommunen mehr als innerhalb der Woche, was letztlich als nutzungsbezogene Preisdifferenzierung interpretiert werden kann, findet doch in aller Regel unter der Woche das Training, am Wochenende hingegen der Spielbetrieb statt.

Verbleibt die Frage der Sporthallenvergabe an Dritte. ${ }^{94}$

Die Frage, ob diese überhaupt die Möglichkeit erhalten, kommunale Sportstätten mitzubenutzen, wird von $50 \%$ der Kommunen grundsätzlich bejaht, darunter wird in $65 \%$ allerdings explizit auf die Nachrangigkeit gegenüber dem Vereinssport hingewiesen.

\footnotetext{
${ }^{93}$ Die beobachteten Versionen der Preisdifferenzierung bezogen sich primär, jedoch nicht ausschließlich, auf Sporthallen.

${ }^{94}$ Hier definiert als Sammelbegriff für alle nicht vereinsorganisiert Sporttreibenden. 
Tabelle 8: Regelungen zur Vergabe kommunaler Sportstätten an Sportvereine und Dritte

\begin{tabular}{|c|c|c|c|}
\hline \multicolumn{2}{|c|}{ Regelungen und Angaben } & Anzahl der Kommunen & in $\%$ \\
\hline \multicolumn{2}{|c|}{ keine Angaben zur Preispolitik } & 20 & 32,8 \\
\hline \multicolumn{2}{|c|}{ Verweis auf separate Gebührenordnung } & 1 & 1,6 \\
\hline \multicolumn{2}{|c|}{ Angaben zu Preispolitik und Vergabe } & 40 & 65,6 \\
\hline \multicolumn{4}{|c|}{ Sportvereine } \\
\hline \multirow[t]{2}{*}{ darunter: } & kostenlose Vergabe & 22 & 55 \\
\hline & Erhebung von Entgelten & 18 & 45 \\
\hline \multicolumn{4}{|l|}{ Dritte } \\
\hline \multicolumn{2}{|c|}{ kein Hinweis auf Vergabe } & 20 & 50 \\
\hline \multicolumn{2}{|c|}{ Hinweis auf Vergabe } & 20 & 50 \\
\hline \multirow[t]{2}{*}{ darunter: } & Nachrangige Vergabe & 13 & 65 \\
\hline & Preisdifferenzierung gegenüber Vereinssport & 9 & 45 \\
\hline
\end{tabular}

45\% jener Kommunen, deren Sportförderrichtlinien eine Vergabe auch an Dritte vorsehen, weisen allerdings darauf hin, daß derart nicht vereinsorganisiert Sporttreibende höhere Gebühren als Sportvereine zu bezahlen haben. ${ }^{95}$

Zusammenfassend kann festgehalten werden, daß über die Hälfte der Gemeinden ihre Sportstätten den Vereinen (nicht in gleichem Maße anderen Sportgruppierungen) kostenfrei überläßt. Interpretiert man die Erhebung symbolischer Mieten oder Pachten als quasi-kostenlose Vergabe, dann ist dieser Anteil um einiges höher.

Eine Unterscheidung zwischen verschiedenen Sportarten erfolgt dabei nicht.

\section{c) Badeanstalten}

Badeanstalten zeichnen sich im Vergleich zu den beiden anderen Sportstättentypen dadurch aus, daß sie für jedermann zugänglich sind, womit die Frage nach der Nutzungsmöglichkeit für Dritte entfältt.

${ }^{95} \mathrm{Vgl}$. hierzu u.a. Stadt Leimen, Anlage 1, Tabelle 1. So wird bspw. für das Training von Erwachsenen in Leimener Stadien ein Preis in Höhe von 5,- DM festgesetzt, die Durchfuhrung eines Erwachsenenwettkampfes schlägt mit 6,- DM etwas höher zu Buche. Handelt es sich bei den betreffenden Sportlern um Nicht-Leimener, dann beträgt das Benutzungsentgelt 20,- bzw. 40,- DM.

Ähnliches ist in Villingen-Schwenningen zu verzeichnen, wo Sportgruppen, die nicht dem örtlichen Sportverband angehören, für die Benutzung der städtischen Sportanlagen zum Zwecke von Veranstaltungen 15,- DM je Stunde entrichten müssen, vgl. Stadt Villingen-Schwenningen, 1985, S. 12.

Der Anteil jener Städte, die von einer nutzerbezogenen Preisdifferenzierung Gebrauch machen, wird die oben genannten $45 \%$ möglicherweise übersteigen, da hier nur Aussagen über jene 20 Kommunen getroffen werden konnten, die in ihren Richtlinien sowohl die Vergabe an verschiedene Sportgruppierungen als auch Benutzungsentgeltregelungen ansprechen. 
Gleichwohl relevant ist die Frage der Gebührenordnung für verschiedene Nutzergruppierungen. Während private Nutzer Entgelte für Schwimmbäder zu entrichten haben, die je nach Ort, Bad und in aller Regel auch Alter und Status des jeweiligen Nutzers differieren, erfahren verschiedene Wassersportvereine zumeist eine gesonderte Behandlung.

Tabelle 9 zeigt, daß die Benutzung seitens der Schwimmsportvereine zu Übungszwecken in 50\% der Fälle kostenfrei, in den anderen 50\% zu Gebühren erfolgt, welche die von Privatpersonen erhobenen Eintrittsgelder in aller Regel weit unterschreiten. ${ }^{96}$

\section{Tabelle 9: Schwimmsportvereine und die Nutzung kommunaler Badeanstalten zu}

Trainings- und Wettkampfzwecken

\begin{tabular}{|c|c|c|c|}
\hline \multicolumn{2}{|c|}{ Regelungen und Angaben } & Anzahl der Kommunen & in $\%$ \\
\hline \multicolumn{2}{|c|}{ keine Angaben in den Richtlinien } & 37 & 60,7 \\
\hline \multicolumn{2}{|c|}{ Angaben zur Gebührenpolitik } & 24 & 39,3 \\
\hline \multirow[t]{2}{*}{ darunter: } & gebührenfrei & 12 & 50 \\
\hline & Erhebung von Benutzungsgebühren & 12 & 50 \\
\hline
\end{tabular}

Abschließend zur Betrachtung der Kostenbeteiligung der Benutzer kommunaler Sportstätten soll darauf hingewiesen werden, daß es sich hierbei um einen Bereich kommunaler Sportpolitik handelt, welcher derzeit in einigen Kommunen überdacht wird.

So hat die jüngere, durch die Finanzknappheit der Kommunen hervorgerufene sportpolitische Diskussion bewirkt, daß vielerorts über eine Einführung oder aber eine Erhöhung der Benutzungsgebühren nachgedacht wird, seien diese nun als Mieten oder auch als Sachkostenbeiträge definiert. ${ }^{97}$

Eine Schnellabfrage des Karlsruher Sportamtes in 23 baden-württembergischen Kommunen Ende 1992 ergab in sechs Fällen beschlossene Kürzungen. Neun Kommunen gaben an, derzeit über mögliche Einnahmeerhöhungen oder Ausgabereduzierungen nachzudenken, in den verbleibenden acht Städten waren Kürzungen kein Thema. ${ }^{98}$

\footnotetext{
${ }^{96}$ Die Stadt Freiburg erhebt mittlerweile für die Benutzung ihrer Badeanstalten durch Erwachsene eine Eintrittsgebühr in Höhe von 6,- DM. Inwieweit es sich hierbei um eine kostendeckende Gebühr handelt soll weiter unten angesprochen werden.

${ }^{97}$ In Freiburg bspw. bleiben zwar dic 0,02 DM/qm und Jahr auch weiterhin bestehen, aber nur furr jene Sportvereine, die für die betreffende Anlage einen langfristigen Pachtvertrag besitzen. Sportgruppen ohne langfristige Pachtverträge, die auf einem städtischen Platz Sport treiben wollen, müssen dafür seit 01.01.1994 eine Miete in Höhe von 5,- DM je Stunde entrichten. In weitaus erheblicherem Maße schlägt schließlich die ebenfalls zum 01.01.1994 in Kraft getretene, oben angeführte Gebührenerhebung für die Benutzung kommunaler Sporthallen zu Buche.

Interessant in diesem Zusammenhang erschien die Aussage eines Stuttgarter Sportamtmitarbeiters, der zu berichten wußte, daß einige Vercine als Folge der auch in Stuttgart durchgeführten Gebührenerhebung die von ihnen beanspruchten Hallenbelegungsstunden heruntergeschraubt hätten und dies dahingehend interpretierte, daß vor Einführung der neuen Gebühren eine gewisse Hamstermentalität bei den Vereinen bestand, welche nun zumindest etwas eingeschränkt worden sei.

${ }^{98} \mathrm{Vgl}$. Stadler, 1993.
} 


\subsubsection{Pflege und Unterhaltung von Sportstätten}

Bezüglich der Pflege und Unterhaltung von Sportstätten lassen sich in der sportpolitischen Praxis zwei prinzipiell verschiedene Praktiken beobachten. Zuweilen werden Pflege und Unterhaltung generell von seiten der Kommune durchgeführt, mitunter werden derartige Aufgaben, insbesondere bei vereinseigenen oder auch langfristig verpachteten Anlagen, auf die Sportvereine übertragen. ${ }^{99}$ Ferner sind Mischformen denkbar.

In 95\% der Fälle, in denen Vereine für die Instandhaltung und Pflege der Anlagen verantwortlich sind, werden von den Kommunen sogenannte Unterhaltungszuschüsse gewährt. Die Bewilligung solcher Gelder hängt zum Teil von der jeweiligen Sportart oder auch davon ab, ob und inwieweit die betreffenden Anlagen gleichfalls alternativ genutzt werden. ${ }^{100}$ Sie sind mitunter als jährliche Pauschalbeträge pro Platz, pro qm sportlich nutzbarer Fläche oder auch als bestimmter Prozentsatz des Betriebskostendefizits definiert.

Tabelle 10: Zuschüsse zur Pflege von Sportanlagen

\begin{tabular}{|c|c|c|c|c|}
\hline \multicolumn{2}{|c|}{ Regelungen und Angaben } & Anzahl der Kommunen & in $\%$ & \\
\hline \multicolumn{2}{|c|}{ keine Angaben } & 10 & 16,4 & \\
\hline \multicolumn{2}{|c|}{ explizite Ablehnung von Zuschüssen } & 3 & 4,9 & 5,9 \\
\hline \multicolumn{2}{|c|}{ Zuschußgewährung } & 48 & 78,7 & 94,1 \\
\hline darunter: & $\begin{array}{l}\text { explizite Unterscheidung nach verschie- } \\
\text { denen Sportanlagen }\end{array}$ & 26 & 54,2 & \\
\hline
\end{tabular}

Zum Zweck eines sportanlagenbezogenen Vergleichs sollen hier aus der Fülle der Einzelregelungen für verschiedene Sportstätten exemplarisch die Förderpraktiken für Rasensportflächen und Leichtathletikanlagen sowie Sondersportanlagen betrachtet werden, wozu in einem ersten Schritt auf die Ergebnisse der Esslinger Studie zurückgegriffen wird.

Die vom Autor durchgeführte Studie kommunaler Sportförderrichtlinien liefert darüber hinaus Angaben zu den in den einzelnen Gemeinden beobachteten Spannen, welche in Tabelle 13 dargestellt werden. ${ }^{101}$

${ }^{99}$ Für Instandhaltung und Pflege städtischer Sporthallen, die von mehreren Sportvereinen oder sonstigen Gruppierungen genutzt werden, sind in den meisten Fällen die Kommunen zuständig.

${ }^{100}$ Mit alternativer Nutzung in diesem Zusammenhang wird in erster Linie eine Nutzung durch Schulen angesprochen.

${ }^{101}$ Die Esslinger Studie beschränkt sich hinsichtlich der Spannen auf Kernsportstätten 
Tabelle 11: Unterhaltungszuschüsse für Rasensportflächen und Leichtathletikanlagen

\begin{tabular}{|c|c|c|c|}
\hline Regelungen und Angaben & Zuschußhöhe & Anzahl der Kommunen & in $\%$ \\
\hline Pflege durch Verein & & 40 & 100 \\
\hline keine Zuschüsse & & 2 & 5 \\
\hline Zuschußgewährung & & 38 & 95 \\
\hline \multirow[t]{4}{*}{ darunter: } & bis $0,5 \mathrm{DM} / \mathrm{qm}$ & 14 & 36,8 \\
\hline & $0,5-0,8 \mathrm{DM} / \mathrm{gm}$ & 14 & 36,8 \\
\hline & $0,8-1,-\mathrm{DM} / \mathrm{qm}$ & 5 & 13,2 \\
\hline & $>1,-\mathrm{DM} / \mathrm{gm}$ & 5 & 13,2 \\
\hline
\end{tabular}

Quelle: Stadt Esslingen, 1990.

Von den 76 innerhalb der Esslinger Studie befragten Kommunen gaben 36 an, die Pflege der vereinseigenen und gepachteten Rasensportflächen und Leichtathletikanlagen erfolge durch die Stadt, in 40 Städten hingegen besorgen die Vereine die Pflege der betreffenden Sportanlagen. Von diesen 40 Kommunen wiederum gewähren $95 \%$ Zuwendungen für den Unterhalt der jeweiligen Sportanlagen, ${ }^{102}$ die in den verschiedenen Kommunen von unter 0,5 DM/qm bis über 1,- DM/qm differieren.

Wesentlich weniger Kommunen fördern den laufenden Unterhalt verschiedener Sondersportanlagen. Ist deren Anteil bei den Tennisplätzen mit $66 \%$ noch vergleichsweise hoch, so wird der laufende Unterhalt der anderen, in Tabelle 12 dargestellten vereinseigenen Sondersportanlagen je nach Sportstättentyp nur in 33 bis $46 \%$ der beobachteten Fälle bezuschußt.

Tabelle 12: Gewährung von Unterhaltungszuschüssen zum Betrieb vereinseigener Sondersportanlagen

\begin{tabular}{|l|l|l|l|l|}
\hline Sportarten & Zuschüsse & in $\%$ & keine Zuschüsse & in \% \\
\hline Tennisplätze & 50 & $66 \%$ & 26 & $34 \%$ \\
\hline Schießanlagen & 35 & $46 \%$ & 41 & $54 \%$ \\
\hline Reitanlagen & 34 & $45 \%$ & 42 & $55 \%$ \\
\hline Bootsanlagen & 29 & $38 \%$ & 47 & $62 \%$ \\
\hline Flugsportanlagen & 25 & $33 \%$ & 51 & $67 \%$ \\
\hline
\end{tabular}

Quelle: Stadt Esslingen, 1990.

Es kann damit festgehalten werden, daß die Unterhaltung von Kernsportstätten im Vergleich zu jener der Sondersportanlagen von den Kommunen als grundsätzlich förderungswürdiger angesehen wird.

${ }^{102}$ Dies entspricht exakt den vom Autor ermittelten Werten. 
Von nicht minderem Interesse ist ein Blick auf die beobachteten Spannen bezüglich der Höhe der Unterhaltungszuschüsse. Tabelle 13 zeigt, daß die Zuschüsse zur laufenden Unterhaltung in den verschiedenen Kommunen zum Teil erheblich differieren.

Tabelle 13: Zur Heterogenität kommunaler Sportstättenunterhaltungszuschüsse

\begin{tabular}{|l|l|l|l|}
\hline Zuschußobjekt & Zuschußregelung & $\begin{array}{l}\text { Zuschußhöhe in DM } \\
\text { pro Platz, Spannen } \\
\text { von ... bis ... }\end{array}$ & $\begin{array}{l}\text { Zuschußhöhe in } \\
\text { DM/qm, Span- } \\
\text { nen von ... bis ... }\end{array}$ \\
\hline $\begin{array}{l}\text { Außensportanlagen gene- } \\
\text { rell }\end{array}$ & generelle Beträge & & $0,3-2,-$ DM \\
\hline \multirow{2}{*}{ Sportplätze } & generelle Beträge & $400-3.000,-\mathrm{DM}$ & $0,25-3,-\mathrm{DM}$ \\
\cline { 2 - 4 } & Naturrasen u./o. Tenne & $550-7.000,-\mathrm{DM}$ & $0,2-2,8 \mathrm{DM}$ \\
\cline { 2 - 5 } & Kunststoff u./o. Asphalt & $550-1.500,-\mathrm{DM}$ & $0,4-1,4 \mathrm{DM}$ \\
\hline Rundbahnen & Naturrasen u./o. Tenne & $1.100-7.000,-\mathrm{DM}$ & \\
\cline { 2 - 5 } & Kunststoff u./o. Asphalt & $550-1.000,-\mathrm{DM}$ & \\
\hline Sporthallen & generelle Beträge & $8.000-15.500$ & $1-200,-\mathrm{DM}$ \\
\cline { 2 - 5 } & $>800$ qm & $1.100-14.300,-\mathrm{DM}$ & \\
\hline Tennisplätze & generelle Beträge & $100-1.100,-\mathrm{DM}$ & $0,3-3,1 \mathrm{DM}$ \\
\cline { 2 - 5 } & Naturrasen u./o. Tenne & $100-3.000,-\mathrm{DM}$ & \\
\cline { 2 - 5 } & Kunststoff u./o. Asphalt & $40-700,-\mathrm{DM}$ & \\
\hline Tennishallen & generelle Beträge & $400-500,-\mathrm{DM}$ & $5-5,5 \mathrm{DM}$ \\
\hline Reitsportanlagen & generelle Beträge & $400-1.000,-\mathrm{DM}$ & $0,2-0,27 \mathrm{DM}$ \\
\hline Reithallen & generelle Beträge & $500-6.500,-\mathrm{DM}$ & $0,7-5,-\mathrm{DM}$ \\
\hline $\begin{array}{l}\text { Schießsportanlagen je } \\
\text { Stand }\end{array}$ & generelle Beträge & $30-600,-\mathrm{DM}$ & $0,2-0,6 \mathrm{DM}$ \\
\hline Kegelanlagen & generelle Beträge & $110-5.000,-\mathrm{DM}$ & \\
\hline Golfplätze & generelle Beträge & $2.000-8.000,-\mathrm{DM}$ & \\
\hline Beleuchtungsanlagen & generelle Beträge & $500-1.345,-\mathrm{DM}$ & \\
\hline Umkleiden u. Duschen & generelle Beträge & $400-1.500,-\mathrm{DM}$ & $1,5-60,-\mathrm{DM}$ \\
\hline
\end{tabular}

So wird bspw. die Unterhaltung von Rasensportflächen und Leichtathletikanlagen in der Stadt Stuttgart mit jährlich 7.000,- DM bezuschußt. ${ }^{103}$ In der Stadt Offenburg beträgt dieser Betrag lediglich 550,- DM jährlich plus einem flächenabhängigem Zuschuß in Höhe von $0,25 \mathrm{DM} / \mathrm{qm} .{ }^{104}$

${ }^{103}$ Vgl. Stadt Stuttgart, 1993, S. 4.

${ }^{104} \mathrm{Vgl}$. Stadt Offenburg, 1991, S. 4. 
Darüber hinaus konnte festgestellt werden, daß bestimmte Kommunen hinsichtlich mehrerer Zuschußobjekte am oberen Ende der Spannbreite angesiedelt sind; letztlich ein Indiz für eine - zumindest bezüglich dieses Förderungsaspekts - vergleichsweise großzügige Sportpolitik. Als Beispiel dient die Stadt Wolfsburg, die mit Unterhaltungszuschüssen in Höhe von jährlich 3,- DM/qm für Rasenflächen, 2,8 DM/qm für Tennebeläge oder 3,1 DM/qm für Tennisplätze jeweils am obersten Ende der Spannenskala rangiert. ${ }^{105}$

Demgegenüber gibt es Kommunen, die für einige Sportanlagen relativ wenig, für andere jedoch vergleichsweise viel Unterhaltungszuschüsse gewähren. Derartige Förderpraktiken zeugen - zumindest was diesen Teilbereich öffentlicher Sportpolitik anbelangt - von einer tendenziellen Präferenz bestimmter Sportarten. Als Beispiel möge die Stadt Offenburg dienen, welche für den Unterhalt gepachteter städtischer Rasenplätze einen Sockelbetrag von 550,- DM und $0,25 \mathrm{DM} / \mathrm{qm}$ nutzbarer Sportfläche, für den Unterhalt von Tennisplätzen hingegen einen Sockelbetrag von DM 1.100,- und 0,3 DM/qm sportlich nutzbarer Fläche gewährt. ${ }^{106}$

Zusammenfassend sollen zwei wesentliche Dinge festgehalten werden. Die Betrachtung der Unterhaltungszuschüsse verdeutlichte abermals, daß sich die Kommunen trotz des Vorhandenseins von Richtlinien hinsichtlich der Detailgestaltung der Sportpolitik stark unterscheiden.

Daneben wird der Eindruck erweckt, daß Kommunen mitunter auch insoweit zwischen verschiedenen Sportarten differenzieren, als sie deren Förderungswürdigkeit unterschiedlich beurteilen. Nun könnte ein hoher Zuschuß für die laufende Unterhaltung natürlich wieder durch einen entsprechend geringeren Zuschuß zu investiven Maßnahmen kompensiert werden. In Fall Offenburg zeigt sich allerdings, daß es sich sowohl bei den Rasen- als auch bei den Tennisplätzen um gepachtete städtische Anlagen handelt. Bauzuschüsse, die in dieser Gemeinde ohnedies unabhängig vom Sportanlagetyp 10\% der Investitionskosten betragen, können die festgestellte Förderpraxis folglich kaum revidieren.

\footnotetext{
${ }^{105}$ Es handelt sich hierbei wohlgemerkt um Höchstgrenzen. Vgl. Stadt Wolfsburg, 1986.

${ }^{106} \mathrm{Vgl}$. Stadt Offenburg, 1991, S. 4. Anzufügen ist, daß sich diese unterschiedliche Handhabung auf die Sockelbeträge bezieht, bezüglich des qm-abhängigen Zuschusses liegt die Stadt Offenburg hinsichtlich beider o.g. Typen von Sportanlagen am unteren Ende der Spannweite.
} 


\subsubsection{Zuschüsse zur Anschaffung von Sport- und Platzpflegegeräten}

Etwa drei Viertel der betrachteten Kommunen gewähren den Sportvereinen Zuschüsse zur Anschaffung von Sport- und Platzpflegegeräten, die verbleibenden $23 \%$ lehnen derartige Subventionen entweder ab oder erwähnen in ihren Sportförderrichtlinien zumindest nichts derartiges.

Der Großteil der mittelgewährenden Kommunen nennt als Förderungsvoraussetzung gewisse Mindestanschaffungskosten, welche wiederum erheblich differieren. ${ }^{107}$ Als Zuschußobjekte gelten vorrangig Sportgeräte wie Trainingsgeräte, Tischtennisplatten, Trampoline, Bodenmatten etc., Sportplatzpflegegeräte und zuweilen auch die Anschaffung eines Computers zur Unterstützung der Verwaltungsarbeit des Vereins.

Als Zuschußhöhe geben die betreffenden Gemeinden einen bestimmten Prozentsatz der Anschaffungskosten, in einigen Fällen auch der zuschußfähigen Kosten des jeweiligen Zuschußobjektes an, der je nach Kommune, seltener auch nach Gegenständen, zwischen 10 bis $50 \%$ variiert.

Tabelle 14: Zuschüsse zur Anschaffung von Sportgeräten

\begin{tabular}{|l|l|l|l|}
\hline Regelung & Zuschußhöhe & Anzahl der Kommunen & in \% \\
\hline keine Angaben & & 13 & 21,3 \\
\hline keine Zuschüsse & & 1 & 1,6 \\
\hline Zuschüsse & & 47 & 77 \\
\hline \multirow{4}{*}{ darunter: } & bis $10 \% \operatorname{der} \mathrm{AK}$ & 1 & 2,1 \\
\cline { 2 - 4 } & $10-20 \% \operatorname{der} \mathrm{AK}$ & 6 & 12,8 \\
\cline { 2 - 5 } & $20-30 \% \operatorname{der} \mathrm{AK}$ & 12 & 25,5 \\
\cline { 2 - 5 } & über 30\% der AK & 15 & 31,9 \\
\cline { 2 - 5 } & keine explizite Nennung & 13 & 27,7 \\
\hline
\end{tabular}

\subsubsection{Förderung des Spitzensports}

Wenngleich die Förderung des Spitzensports grundsätzlich Bundes- und Länderangelegenheit ist und die Gemeinden tendenziell Aufgaben auf dem Gebiet des Breitenund Freizeitsports wahrnehmen, existieren einige Überschneidungen. Kommunale Förderung des Spitzen- oder Leistungssports erfolgt - abgesehen vom Berufssport, der

${ }^{107}$ Die Stadt Leimen nennt als Mindestkosten des jeweiligen Geräts 100,- DM, vgl. Stadt Leimen, 1992, S. 11. Die Stadt Heidelberg hingegen fördert lediglich den Kauf von Sportgeräten mit einem Mindestwert von 5.000,- DM. Vgl. Stadt Heidelberg, 1991, S. 4. 
in Abschnitt 2.1.7. anhand des Beispiels Profifußball näher betrachtet wird - auf verschiedenen Ebenen.

Zunächst spielt in einigen Kommunen auch der sportliche Erfolg einer Mannschaft eine gewisse Rolle bei der Vergabe der zumeist knappen Hallenkapazitäten. ${ }^{108}$ Neben dieser indirekten Förderung durch bevorzugte Überlassung von Sportstätten beinhalten die Sportförderrichtlinien eines Großteils der untersuchten Kommunen auch Abschnitte, die explizit leistungsorientiert sind und die Höhe der jeweiligen direkten $\mathrm{Zu}$ schüsse explizit vom sportlichen Erfolg des betreffenden Vereins bzw. der betreffenden Mannschaft abhängig machen.

Hierzu gehören bspw. die Fahrtkosten, Tagegelder und sonstigen Pauschalen, die 49 der betrachteten 61 Kommunen ihren Vereinen für die Teilnahme an höherwertigen sportlichen Wettkämpfen gewähren. ${ }^{109}$ Als höherwertig in diesem Sinne werden zumeist Teilnahmen an Welt- Europa- und Deutschen oder zumindest überregionalen Meisterschaften eingestuft. Die Höhe dieser leistungsorientierten Zuschüsse richtet sich zumeist nach den entsprechenden Bundesbahnkosten 2. Klasse oder aber bestimmten Kilometergeldern.

\section{Tabelle 15: Förderung des Leistungs- und Spitzensports}

\begin{tabular}{|l|l|l|l|}
\hline Zuschußobjekt & Regelung & Anzahl der Kommunen & in \% \\
\hline \multirow{2}{*}{ Fahrtkosten, Startgelder u.ä. } & nein oder keine Angaben & 12 & 19,7 \\
\cline { 2 - 4 } & Gewährung & 49 & 80,3 \\
\hline \multirow{2}{*}{$\begin{array}{l}\text { Ausfallbürgschaften oder Zuschüs- } \\
\text { se zu bedeutenden Sportveran- } \\
\text { staltungen }\end{array}$} & nein oder keine Angaben & 24 & 39,3 \\
\cline { 2 - 4 } & Gewährung & 37 & 60,7 \\
\hline $\begin{array}{l}\text { Spielbetrieb in Abhängigkeit von } \\
\text { der Ligenzugehörigkeit }\end{array}$ & keine Angaben & 52 & 85,2 \\
\cline { 2 - 4 } & Gewährung & 9 & 14,8 \\
\hline
\end{tabular}

Im Zusammenhang mit der Veranstaltung überregionaler Wettkämpfe weisen etwa $60 \%$ der Kommunen auf die Möglichkeit der Gewährung von Veranstaltungszuschüssen oder der Übernahme von Ausfallbürgschaften hin. Laut Studie der Stadt Esslingen liegt dieser Anteil sogar bei 87\%.

Einige Städte gewähren ihren Vereinen darüber hinaus bestimmte Mannschaftsbeträge für den Spielbetrieb, die ebenfalls von der jeweiligen Spielklasse abhängen.

\footnotetext{
${ }^{108}$ Hierauf angesprochen antworteten einige Sportamtsmitarbeiter, daß neben den weiter oben genannten Kriterien auch der sportliche Erfolg einer Mannschaft eine Rolle spielen könne.

${ }^{109}$ Einschränkend soll hierzu vermerkt werden, daß ein Teil der zuschußgewährenden Kommunen derartige Mittel ausschließlich für den Amateursport bereitstellt.
} 


\subsubsection{Personelle Förderungsaspekte}

In den Richtlinien fast aller betrachteten Gemeinden wird der Förderung der Jugendarbeit großes Gewicht beigemessen. Neben indirekten Zuwendungen in Form bevorzugter, verbilligter oder zumeist kostenloser Überlassung von Sportstätten gewähren 49 Kommunen den Vereinen direkte Zuschüsse in Abhängigkeit von der Anzahl der jugendlichen Mitglieder (unter 18 Jahren). Diese Pro-Kopf-Zuschüsse differieren in jenen Kommunen, die hierzu konkrete DM-Beträge nennen, zwischen 2,5 DM (Solingen) und 30,- DM (Ulm) je Jugendlichem je Jahr (Ulm). Die Stadt Heidelberg fördert den Jugendsport zusätzlich auf eine ungewöhnliche Art und Weise, indem die Zuschüsse für Investitionen an die Altersstruktur der Vereine gekoppelt werden. ${ }^{110}$ Zum Vergleich, laut Umfrage der Stadt Esslingen gewähren 75\% der Kommunen sogenannte Jugendzuschüsse.

Weit verbreitet ist auch die finanzielle Unterstützung von Übungsleitern, die von 39 Kommunen praktiziert wird. In diesem Zusammenhang werden Beträge entweder pro Übungseinheit oder Stunde in Höhe von 1,- bis 8,- DM oder aber je Übungsleiter in Höhe von 200,- bis 500,- DM jährlich unter Beachtung von Höchstbeträgen ausbezahlt. Der Esslinger Studie zufolge gewähren 56 Städte Übungsleiterzuschüsse, abgelehnt wird dieser Förderaspekt von 20 der untersuchten Kommunen.

\section{Tabelle 16: Personenabhängige Förderungen}

\begin{tabular}{|l|l|l|l|}
\hline Zuschußobjekt & Regelung & Anzahl der Kommunen & in \% \\
\hline \multirow{2}{*}{ Jugendzuschüsse } & nein bzw. keine Angaben & 12 & 19,7 \\
\cline { 2 - 4 } & Zuschußgewährung & 49 & 80,3 \\
\hline \multirow{2}{*}{ Übungsleiterzuschüsse } & nein bzw. keine Angaben & 22 & 36,1 \\
\cline { 2 - 4 } & Zuschußgewährung & 39 & 63,9 \\
\hline \multirow{2}{*}{$\begin{array}{l}\text { Allg. Pro-Kopf- oder } \\
\text { Grundzuschüsse }\end{array}$} & nein bzw. keine Angaben & 40 & 65,6 \\
\cline { 2 - 4 } & Zuschußgewährung & 21 & 34,4 \\
\hline \multirow{2}{*}{$\begin{array}{l}\text { Zuschuß je Absolvent des } \\
\text { Deutschen Sportabzeichens }\end{array}$} & nein bzw. keine Angaben & 58 & 95,1 \\
\cline { 2 - 4 } & Zuschußgewährung & 3 & 4,9 \\
\hline
\end{tabular}

Seltener hingegen sind allgemeine Pro-Kopf-Zuschüsse oder Grundzuschüsse pro Verein anzutreffen, die lediglich von 21 der untersuchten Gemeinden ausbezahlt werden und eine Größenordnung von 1,- bis 2,- DM je Mitglied und/oder 100,- bis 400,-

${ }^{110}$ Die Investitionszuschüsse in Heidelberg belaufen sich grundsätzlich auf bis zu $30 \%$ der zuschußfähigen Aufwendungen. Liegt der Anteil der Mitglieder bis zum 25. Lebensjahr allerdings unter $20 \%$, so werden nur bis zu $25 \%$ der zuschußfähigen Aufwendungen bezuschußt. Vgl. Stadt Heidelberg, 1991, S. 3. 
DM je Verein betragen. Dieses Ergebnis deckt sich in etwa mit jenem der Esslinger Abfrage. So kam auch diese Studie zum Schluß, daß lediglich 20 Städte bzw. $26 \%$ eine Grundförderung gewähren, 56 Städte einen solchen Grundzuschuß demgegenüber ablehnen.

Einige wenige Städte fördern darüber hinaus auch den Behindertensport durch ProKopf-Beträge oder Pauschalzuschüsse für Behindertensportvereine. ${ }^{111}$ Gleiches gilt für das Ablegen des Deutschen Sportabzeichens, welches laut Richtlinien von drei Gemeinden mit 1,5 bis 4,- DM je Abzeichen gefördert wird.

\subsubsection{Ehrungen und Jubiläumsgaben}

Zum Abschluß der Betrachtung einzelner Positionen kommunaler Sportförderrichtlinien soll auf einen Förderungsaspekt eingegangen werden, der zwar unter finanziellen Gesichtspunkten vernachlässigbar ist, hier jedoch aus Gründen der Vollständigkeit aufgeführt werden soll.

40 der untersuchten Sportförderrichtlinien zeichnen sich dadurch aus, daß sie die Vereine oder auch einzelne Sportler aus bestimmten Anlässen ehren. Dies geschieht zum einen durch die Verleihung von Medaillen oder Urkunden aufgrund erbrachter Leistungen oder im Zusammenhang mit bestimmten Veranstaltungen. Ebenfalls gewährt ein Großteil der Städte seinen Sportvereinen zu bestimmten Jubiläen sogenannte Jubiläumsgaben. In den meisten Fällen werden diese nach jeweils 25 Jahren des Bestehens ausbezahlt und betragen in aller Regel 10,- DM je Jahr.

\subsubsection{Die Förderung einzelner Sportarten}

Grundsätzliche Aussagen zu etwaigen, nach verschiedenen Sportarten differenzierenden Förderungspraktiken können aus der Betrachtung kommunaler Sportförderrichtlinien kaum abgeleitet werden.

Die Betrachtung der Investitionszuschüsse offenbarte zwar in einigen Fällen höhere Maximalsätze für Kernsportstätten bzw. -arten, aus den oben aufgeführten Gründen kann hieraus jedoch keineswegs pauschal auf eine verstärkte Förderung dieser Sportarten geschlossen werden.

${ }^{11}$ Es sind dies im einzelnen die Städte Bad Salzuflen, Freiburg, Leverkusen, Neumünster und Neustadt am Rübenberge. 
Anders im Zusammenhang mit Unterhaltungszuschüssen. Während in $95 \%$ jener Kommunen, in denen die Pflege von Rasensportflächen und Leichtathletikanlagen grundsätzlich durch die Vereine durchgeführt wird, Unterhaltungszuschüsse für derartige Kernsportstätten gewährt werden, ist der prozentuale Anteil bezuschussender Kommunen bei den Sondersportanlagen deutlich geringer.

Es konnten allerdings auch Fälle angetroffen werden, in denen eine vergleichsweise starke Förderung zur laufenden Unterhaltung bestimmter Sondersportanlagen gewährt wird.

Die weiteren, innerhalb der Richtlinien aufgeführten Förderungsaspekte kannten keinerlei sportartenbezogene Differenzierungen.

\subsubsection{Gemeindliche Sportförderrichtlinien - ein Instrument zur Verein- heitlichung kommunaler Sportpolitik?}

Die Betrachtung kommunaler Sportförderrichtlinien hat gezeigt, daß die Kommunen den organisierten Sport durch eine Vielzahl kaum überschaubarer Einzelmaßnahmen fördern. Kommunale Vereinsförderung beschränkt sich keineswegs auf die Überlassung von Sportanlagen und die Gewährung von Investitionszuschüssen. Vielmehr werden dem organisierten Sport eine Reihe weiterer, laufenden Zwecken dienende Gelder zugestanden.

Eine Ausrichtung kommunaler Sportförderrichtlinien an den Empfehlungen des Deutschen Städtetages zeigt sich darin, daß sich die in den Empfehlungen des Deutschen Städtetages genannten Fördermaßnahmen im Großteil der analysierten Sportförderrichtlinien wiederfinden.

Daraus jedoch auf eine Vereinheitlichung kommunaler Sportpolitik zu schließen wäre voreilig, da es etliche Punkte gibt, die von einigen Kommunen gefördert werden, von anderen wiederum nicht. Auch zwischen Kommunen, die die grundsätzliche Frage der Förderungswürdigkeit eines bestimmten Aspekts zustimmend beantwortet haben, bestehen zum Teil große Unterschiede bezüglich der Zuschußhöhen.

Zusammenfassend kann festgehalten werden, daß die Empfehlungen des Deutschen Städtetages genauso wenig zu einer Vereinheitlichung qualitativer Aspekte kommunaler Sportförderung geführt haben wie auch schon die weiter oben genannten Orientierungshilfen hinsichtlich kommunaler Sportstättenpolitik.

Es kann ferner resümiert werden, daß die Betrachtung der Richtlinien eine unterschiedlich hohe Förderung des Sports in einzelnen Kommunen erwarten läßt. Es wird Aufgabe von Abschnitt 2.1.7.2. sein, dies zu untersuchen. 


\subsubsection{Exkurs: Kommune und Profifußball}

Wie an anderer Stelle bereits erwähnt, rät der Deutsche Städtetag den Kommunen in den Empfehlungen zur kommunalen Sportförderung von einer Förderung des Profisports ab. Berufssport könne zwar aus Gesichtspunkten wie bspw. der Wirtschaftsförderung oder der Öfentlichkeitsarbeit unterstützt werden, nicht jedoch aus dem kommunalen Sportetat. Für die dem Berufssport zur Verfügung gestellten Sportstätten, so die Empfehlungen des Deutschen Städtetages weiter, sollten Entgelte erhoben werden. ${ }^{112}$

Um die Frage beantworten zu können, ob und inwieweit die Kommunen den Berufssport auch innerhalb des Sportetats netto fördern, soll im folgenden Abschnitt, stellvertretend für den Profisport, die kommunale Förderung des Bundesligafußballs untersucht werden. ${ }^{113}$

Eine Betrachtung der 18 derzeitigen Bundesligaclubs zeigt, daß bis auf den 1. FC Kaiserslautern alle Vereine ihre Spiele in städtischen Stadien austragen, 114 wofür sie laut Aussage des Deutschen Städtetages Benutzungsentgelte zu entrichten haben, die sich im allgemeinen zwischen $5-15 \%$ der Zuschauernettoeinnahmen bewegen ${ }^{115}$ und zuweilen mit jährlichen Mindestbeiträgen versehen sind.

Die jeweiligen Stadien selbst sind wiederum Bestandteil der Kommunalhaushalte und werden in aller Regel im Einzelplan 5, Abschnitt 560 verwaltet. ${ }^{116}$

Will man nun überprüfen, ob diese Bereitstellung der Stadien eine Nettoförderung des Bundesligafußballs - und falls ja, in welchem Ausmaß - darstellt, so wird ein Vergleich der in diesem Zusammenhang entstehenden Ausgaben und Einnahmen erforderlich. Von einer Nettoförderung kann demzufolge dann gesprochen werden, wenn die Benutzungsentgelte und weitere, mit der Vermietung des Stadions einhergehenden Einnahmen nicht zur Deckung der gesamten Ausgaben hinreichen.

Beim Versuch, den betriebswirtschaftlichen Kostendeckungsgrad einzelner Fußballbundesligastadien zu ermitteln, stößt man allerdings bald auf Probleme. ${ }^{117}$

\footnotetext{
${ }^{112} \mathrm{Vgl}$. Deutscher Städtetag, Empfehlungen zur kommunalen Sportförderung, S. 8.

${ }^{113}$ Die exemplarische Betrachtung des Fußballsports wurde deshalb gewählt, weil es sich hierbei unbestritten um die mit Abstand populärste Profisportart in der Bundesrepublik Deutschland handelt.

114 Stand Saison 1993/94.

${ }^{115}$ Die Zuschauemettoeinnahmen setzen sich zusammen aus den Zuschauerbruttoeinnahmen, reduziert um die Umsatzsteuer und kleineren Beiträgen an die Sepp Herberger Stiftung.

${ }^{116}$ Einige Kommunen führen das jeweilige Stadion gemeinsam mit weiteren Anlagen in einem Unterabschnitt des HH-Abschnitts 56, Sportstätten, andere Kommunen hingegen haben innerhalb dieses Abschnitts einen separaten Unterabschnitt für das betreffende Stadion eingerichtet.
} 
Zum einen wird eine Erfassung der entstandenen Ausgaben dort schwierig, wo das jeweilige Stadion lediglich Bestandteil eines Sammelpostens ist. Zum anderen kennen öffentliche Haushalte auch bei solchen Großsportstätten mitunter keine Abschreibungen, schon gar keine kalkulatorischen Abschreibungen vom Wiederbeschaffungswert und darüber hinaus keine kalkulatorische Zinsen, die, wollte man eine Kostendeckung im betriebswirtschaftlichem Sinne erreichen, notwendige Bestandteile einer solchen Kostenrechnung wären.

Nichtsdestotrotz sollen im weiteren Verlauf die Ausgaben und Einnahmen von vier Bundesligastadien einander gegenübergestellt werden, um die Frage nach der Nettoförderung des Profifußballs somit zumindest annäherungsweise beantworten zu können. $\mathrm{Zu}$ diesem Zweck werden die Verhältnisse in den drei derzeitigen baden-württembergischen Bundesligastädten Freiburg, Karlsruhe und Stuttgart sowie Mönchengladbach betrachtet.

\section{a) Das Freiburger Dreisamstadion}

Zunächst zum Sport Club Freiburg, der seine Heimspiele im städtischen Dreisamstadion austrägt. Die Ausgaben und Einnahmen, die der Stadt in Zusammenhang mit diesem Stadion entstehen, sind kaum exakt zu ermitteln, da die Stadt Freiburg das Dreisamstadion neben einem weiteren größeren Stadion und der ebenfalls dem Profisport dienenden Eishalle ${ }^{118}$ gemeinsam in einem Unterabschnitt 5620, "Stadien und Sportplätze - Unternehmerischer Bereich", verwaltet. Freiburg unterscheidet sich von den anderen untersuchten Bundesligastädten des weiteren durch folgendes:

Die Stadt erhebt keine zuschauerzahlenabhängige Benutzungsgebühr, sondern behandelt den Sport Club Freiburg wie jeden anderen Sportverein und erhebt eine Pacht für die sportlich nutzbaren Flächen in Höhe von $0,02 \mathrm{DM} / \mathrm{qm}$ und Jahr sowie 4\%ige Erbbauzinsen auf den Bodenwert in Höhe von 5,- DM je qm, also hierbei de facto 0,2 $\mathrm{DM} / \mathrm{qm}$. Hieraus dürften allenfalls Einnahmen in einer Größenordnung von maximal DM 5.000 jährlich resultieren, denn von den DM 62.000, die dieser Unterabschnitt für das Haushaltsjahr 1994 ausweist, entfallen allein über DM 50.000 DM auf den örtlichen Eishockeyverein.

Weitere mögliche, stadionbezogene Einnahmen aus Werbung und Gastronomie sind nicht Bestandteil des betreffenden Haushaltsunterabschnitts.

${ }^{117}$ Kostendeckungsgrad hier als prozentualer Anteil der Einnahmen an den gesamten, für das jeweilige Stadion ausgewiesenen Ausgaben. Zwischen den Begriffen Ausgaben, Aufwendungen und Kosten wird im Rahmen kommunaler Haushalte nicht unterschieden.

${ }^{118}$ Der EHC Freiburg gehörte bis zum Zwangsabstieg 1993 der ersten Eishockeybundesliga an. 
Auch auf der Ausgabeseite unterscheidet sich Freiburg von den anderen Bundesligisten. Während die Pflege fast aller Stadien durch die jeweilige Kommune erfolgt, ist in Freiburg primär der Verein für den laufenden Unterhalt verantwortlich. Damit wird von seiten der Stadt auch die geringe Miete erklärt.

Tabelle 17: Zum Ausweis des Freiburger Dreisamstadions im Verwaltungshaushalt der Stadt Freiburg

\begin{tabular}{|c|c|c|c|c|c|}
\hline \multicolumn{6}{|c|}{ Unterabschnitt 5620, Stadien und Sportplätze - Unternehmerischer Bereich } \\
\hline \multirow[b]{2}{*}{ Ausgaben/Einnahmen } & \multirow[b]{2}{*}{ Bezeichnung } & \multicolumn{3}{|l|}{ Ansatz } & \multirow{2}{*}{$\begin{array}{l}\text { Rechnung } \\
1991\end{array}$} \\
\hline & & 1994 & 1993 & 1992 & \\
\hline \multicolumn{6}{|l|}{ Einnahmen } \\
\hline darunter: & $\begin{array}{l}\text { Miete, Pacht aus bebauten } \\
\text { Grundstücken }\end{array}$ & 62.000 & 62.000 & 62.000 & 62.069 \\
\hline \multicolumn{6}{|l|}{ Ausgaben } \\
\hline \multirow[t]{5}{*}{ darunter: } & Personalausgaben & 0 & 0 & 59.160 & 0 \\
\hline & $\begin{array}{l}\text { Unterhaltung Grundstücke } \\
\text { und bauliche Anlagen }\end{array}$ & 167.500 & 140.700 & 151.090 & 324.997 \\
\hline & Abschreibungen & 236.930 & 236.930 & 222.230 & 222.230 \\
\hline & $\begin{array}{l}\text { Verzinsung des Anlagekapi- } \\
\text { tals }\end{array}$ & 286.890 & 286.890 & 274.840 & 274.840 \\
\hline & $\begin{array}{l}\text { Sonstiges (Zusammenfas- } \\
\text { sung kleinerer Positionen) }\end{array}$ & 62.350 & 60.780 & 41.710 & 43.460 \\
\hline \multicolumn{2}{|l|}{ Gesamtausgaben } & 753.670 & 725.300 & 749.030 & 865.527 \\
\hline \multicolumn{2}{|l|}{ Gesamteinnahmen } & 62.000 & 62.000 & 62.000 & 62.069 \\
\hline \multicolumn{2}{|l|}{ Zuschußbedarf } & 691.670 & 663.300 & 687.030 & 803.458 \\
\hline
\end{tabular}

Quelle: Haushaltsplan der Stadt Freiburg im Breisgau 1993/94, Teil 1, S. 247.

Eine Abschreibung des Stadions und eine Verzinsung des Anlagekapitals erfolgen ebenfalls nicht; der Haushalt beinhaltet zwar derlei Gruppienungsziffern, diese beziehen sich aber lediglich auf das Eisstadion.

Die einzige, aus dem Haushalt ersichtliche Gruppierungsziffer, die auch bezogen auf den Sport Club Freiburg von größerer Bedeutung ist, ist die Unterhaltung der Grundstücke und baulichen Anlagen, innerhalb der - so das Freiburger Sportamt - ein größerer aber nicht näher definierter Teilbetrag auf den Sport Club Freiburg entfällt. ${ }^{119}$ Eine Zusammenstellung der tatsächlichen Ausgaben und Einnahmen des Freiburger Dreisamstadions ist somit kaum möglich. $\mathrm{Zu}$ erwähnen sind allerdings trotz des Verzichts auf einen Ansatz von Abschreibungs- und Zinsaufwendungen die jüngsten, auf-

${ }^{119}$ Auskunft des Freiburger Sportamtes. 
grund der erfolgreichen sportlichen Entwicklung im Stadion durchgeführten Investitionen.

So wurde vor der Saison 1993/94 eine neue Gegentribüne für etwa DM 1,3 MIO erbaut, die allerdings vom Verein finanziert wurde. Die Stadt hingegen trug die Kosten der Installierung einer Flutlichtanlage in Höhe von $\mathrm{DM} \pm 1,5 \mathrm{MIO}$.

Im Sommer 1994 wurde die Haupttribüne um 3.000 Zuschauerplätze aufgestockt, die endgültigen Kosten dieser Baumaßnahme liegen noch nicht vor, bewegen sich jedoch etwa im Bereich von DM 7 MIO, wovon die Stadt und das Land jeweils die Hälfte tragen. Die jüngste Investition in das Freiburger Dreisamstadion stellt der im Frühjahr 1995 begonnene Bau einer gänzlich neuen Südtribüne dar, die zum Beginn der Spielzeit 1995/96 fertiggestellt sein soll.

Zusammenfassend kann festgehalten werden: Die Stadt fördert den Sport Club Freiburg durch die genannten Investitionsmaßnahmen und die sehr günstige Bereitstellung des Stadions, wofür dann andererseits auch vergleichsweise wenig Unterhaltungsaufwand anfällt sowie einigen kleineren zusätzlichen Mitteln. ${ }^{120}$ Dies läuft de facto auf eine Nettoförderung hinaus, die allerdings kaum zu beziffern ist.

Wollte man bspw. die unlängst durchgeführten Investitionsmaßnahmen auch im Verwaltungshaushalt erfassen, dann müßten dies bei einer unterstellten Nutzungsdauer von 40 Jahren zu Abschreibungen in Höhe von mindestens DM 200.000 jährlich führen. ${ }^{121}$ Ginge man vom Wiederbeschaffungswert aus und würde darüber hinaus kalkulatorische Zinsen auf das Anlagekapital berücksichtigen, wäre dieser Betrag selbstverständlich um einiges höher.

Es bleibt abzuwarten, inwieweit im Falle einer Etablierung des SC Freiburg in der ersten Fußballbundesliga und evtl. weiteren Investitionen in das Stadion zukünftig ein eigenständiger Haushaltsunterabschnitt Dreisamstadion eingerichtet werden wird.

\section{b) Das Karlsruher Wildparkstadion}

Etwas exakter lassen sich die Ausgaben abschätzen, die der Stadt Karlsruhe im Zusammenhang mit der Unterhaltung des städtischen Wildparkstadions entstehen.

Die Stadt hat in ihrem Haushalt einen eigenen Unterabschnitt 5600, "Wildparkstadion", eingerichtet und erhebt vom Karlsruher Sport Club eine Stadionmiete in Höhe von 8\% der Zuschauernettoeinnahmen, mindestens jedoch DM 250.000

${ }^{120}$ Hierzu gehören neben einem sechsstelligen Betrag zur Erfüllung von Sicherheitsauflagen des DFB auch bspw. ein ZuschuB in Höhe von 30.000,- DM zum Öffentlichen-Personen-Nahverkehr bezüglich der Verkehrsregelungen im Zusammenhang mit Bundesligaspielen oder durchgefuhrte kleinere bauliche Maßnahmen wie Umzäunung des Areals (siehe Gruppierungsziffer Unterhaltung der Grundstücke und baulichen Anlagen).

${ }^{121}$ Ausgehend von bisherigen jüngeren Investitionskosten in Höhe von etwa DM 8,5 Millionen. 
jährlich. ${ }^{122}$ Daneben wird, im Gegensatz zu Freiburg, die laufende Pflege des Stadions von der Stadt durchgeführt. Ferner handelt es sich beim Karlsruher Wildparkstadion um eine Arena, die zwar weitestgehend, aber nicht ausschließlich vom Karlsruher Sport Club genutzt wird. In dem Maße, indem auch alternative Nutzungen erfolgen, kann selbstverständlich nicht resümiert werden, jede Mark für das Wildparkstadion sei eine Mark für den Profifußball. Analog zur Stadt Freiburg verzichtet auch Karlsruhe auf den Ansatz von Abschreibungs- und Zinsaufwendungen und weist keine Einnahmen aus Werbung und Bewirtung aus.

Einen Überblick über den absoluten und relativen Zuschußbedarf sowie den "Kostendeckungsgrad"123 des Unterabschnitts 5600 gibt Tabelle 20.

Tabelle 18: ZuschuBbedarf zu den laufenden Einnahmen und Ausgaben des Karlsruher Wildparkstadions von 1977 bis 1994 in TDM

\begin{tabular}{|l|l|l|l|l|l|l|l|l|l|l|l|}
\hline Einn./Ausgaben & \multicolumn{4}{|l|}{ Rechnungsergebnisse } & \multicolumn{4}{l|}{ HH-Ansätze } \\
\hline Jahr & 1977 & 1979 & 1981 & 1983 & 1985 & 1987 & 1989 & 1991 & 1992 & 1993 & 1994 \\
\hline Ausgaben & 281 & 332 & 548 & 443 & 464 & 609 & 536 & 632 & 694 & 666 & 697 \\
\hline Einnahmen & 63 & 72 & 63 & 95 & 88 & 109 & 112 & 114 & 112 & 221 & 262 \\
\hline Zuschußbedarf & 219 & 260 & 485 & 349 & 376 & 463 & 422 & 518 & 582 & 445 & 435 \\
\hline Zuschußbedarf in \% & 78 & 78 & 89 & 79 & 81 & 82 & 79 & 82 & 84 & 67 & 62 \\
\hline Kostendeckung in \% & 22 & 22 & 11 & 21 & 19 & 18 & 21 & 18 & 16 & 33 & 38 \\
\hline
\end{tabular}

Quelle: Haushaltspläne der Stadt Karlsruhe von 1979 bis $1994 .{ }^{124}$

Das Karlsruher Wildparkstadion "kostete" die Stadt in den letzten 16 Jahren laufend netto zwischen DM 219.000 (1977) bis DM 752.000 (1992). Die jeweiligen prozentualen Zuschußbeträge lagen zumeist zwischen etwa 80 bis $90 \%$, Kostendeckung konnte damit also in einer Größenordnung von nur etwa 10 bis $20 \%$ erreicht werden. Lediglich in den Haushaltsansätzen für die Jahre 1993 und 1994 wird von einer höheren Kostendeckung im Umfang von 33 bzw. 38\% ausgegangen, was maßgeblich auf die stark angestiegenen Mindestmieteinnahmen zurückzuführen ist.

Dieser höhere Kostendeckungsgrad in den Haushaltsansätzen 1993 und 1994 verringerte sich allerdings beträchtlich, wenn man Abschreibungs- und Zinsaufwendungen

122Dieser jährliche Mindestbetrag hat sich in den letzten Jahren stark erhöht. So betrug er noch im Jahr 1990 DM 84.000 und hat sich damit innerhalb von 4 Jahren verdreifacht.

${ }^{123}$ Hierbei handelt es sich aufgrund der nicht ausgewiesenen kalkulatorischen Aufwendungen um einen fiktiven Kostendeckungsgrad der laufenden Ausgaben, der tatsächliche Kostendeckungsgrad liegt niedriger.

${ }^{124}$ Eine detaillierte Aufschlüsselung der einzelnen Ausgabe- und Einnahmepositionen findet sich in Anhang 2. 
für jene Investitionsmaßnahmen ansetzen würde, die in den letzten Jahren im Karlsruher Wildparkstadion durchgeführt wurden.

So wurde in diesem Stadion unlängst eine neue Haupttribüne errichtet, welche - die endgültige Abrechnung liegt noch nicht vor - etwa DM 45 MIO kostete. Davon übernahm das Land etwa knapp ein Drittel. Weitere Investitionsmaßnahmen seitens der Stadt, die im Zusammenhang mit den Sicherheitsbestimmungen der UEFA für internationale Begegnungen erbracht wurden, sind in diesem Betrag nicht enthalten.

Es ist offensichtlich, daß der Ansatz von Abschreibungen und Zinsen einer kommunalen Investition in dieser Größenordnung den Kostendeckungsgrad wahrscheinlich unter $20 \%$, eventuell sogar unter $10 \%$ drücken würde. ${ }^{125}$

Als Ergebnis kann festgehalten werden, daß die Stadt Karlsruhe den ortsansässigen KSC und damit den Profifußball mit erheblichen Mitteln aus dem Sportetat fördert, von einer kostendeckenden Stadionmiete kann keineswegs die Rede sein.

\section{c) Das Stuttgarter Gottlieb-Daimler-Stadion}

Der VfB Stuttgart trägt seine Heimspiele ebenfalls in einem städtischen Stadion, dem Gottlieb-Daimler-Stadion aus, für welches die Stadt einen Unterabschnitt 5610, "Gottlieb-Daimler-Stadion mit Nebenanlagen", eingerichtet hat. Für die Nutzung dieser von seiten der Stadt unterhaltenen Arena bezahlt der VfB Stuttgart eine Pacht in Höhe von 7,5\% der Zuschauernettoeinnahmen.

Wie im Fall der Stadt Karlsruhe können die mit dem Stadion verbundenen Ausgaben nicht ausschließlich als Förderung des Profifußballs interpretiert werden, finden doch auch hier weitere Veranstaltungen wie bspw. die Leichtathletikweltmeisterschaft 1993 statt. Hinzu kommt, daß sich dieser Haushaltsunterabschnitt auch auf einige Nebenanlagen, genauer, auf zwei weitere Sportstätten und ein zusätzliches Stadion erstreckt. Da es sich hierbei im Vergleich zum Gottlieb-Daimler-Stadion um wesentlich weniger kostenintensive Sportanlagen handelt und ferner davon ausgegangen werden kann, daß der Großteil der Nutzung des Gottlieb-Daimler-Stadions auf den VfB Stuttgart entfält, soll die folgende Darstellung der Ausgaben und Einnahmen dieser Haushaltsstelle trotz kleiner Einschränkungen primär als Förderung des VfB Stuttgart angesehen werden.

Eine weitere Parallele zur Betrachtung des Karlsruher Wildparkstadions ergibt sich dadurch, daß auch in Stuttgart kürzlich in diese Großarena investiert wurde.

\footnotetext{
${ }^{125}$ Ein Vergleich der angesetzten Einnahmen 1994 in Höhe von DM 262.000 mit den angesetzten Ausgaben in Höhe von DM 697.300 und zusätzlich unterstellten kalkulatorischen Aufwendungen in Höhe von nur DM 1 MIO - realistischer wäre wohl ein weitaus höherer Betrag - würde zu einem Kostendeckungsgrad in Höhe von etwa $15 \%$ führen.
} 
Innerhalb dieser Investitionsmaßnahme wurde das Stadion mit einem neuen Dach versehen, daneben erfolgte eine Umwandlung von Steh- in Sitzplätze, was zu Gesamtkosten in Höhe von etwa DM 55 MIO führte. Davon trug das Land Baden-Württemberg DM 25 MIO, die Daimler-Benz AG beteiligte sich als Gegenleistung für die Umbenennung - das Stadion hieß vorher Neckarstadion - mit DM 7,5 MIO an den Kosten und die Stadt selbst kam für die restlichen DM 22,5 MIO auf.

Laut Aussage des Stuttgarter Sportamtes war es oberstes Ziel der Stadt, den kommunalen Haushalt durch diese Investition per Saldo nicht zu belasten, mit anderen Worten, Kostendeckung zu erreichen. Zu diesem Zweck erhöhte die Stadt die Stadionmiete für den ortsansässigen $\mathrm{VfB}$ auf die oben genannten $7,5 \%$ und führte einen sogenannten Stadiongroschen ein, ${ }^{126}$ mit dem die Besucher zusätzlich an der Finanzierung beteiligt werden sollen. Schließlich wurden weitere Vermarktungsmaßnahmen innerhalb des Stadions ergriffen, um die Zins- und Tilgungsverpflichtungen des zur Durchführung der Investition aufgenommenen Darlehens bedienen zu können.

Nun unterscheidet sich der das Stadion betreffende Unterabschnitt im Haushalt der Stadt Stuttgart ganz elementar von jenem der Stadt Karlsruhe. So werden in Stuttgart sowohl Werbeeinnahmen als auch kalkulatorische Aufwendungen in Ansatz gebracht, was die Beantwortung der Frage, inwieweit die Kostendeckung ein realistisches Ziel ist, immens erleichtert.

Tabelle 19 zeigt eine wesentlich exaktere Berechnung der gesamten, mit dem Unterhalt eines Großstadions (incl. der genannten Nebenanlagen) einhergehenden Ausgaben und Einnahmen. ${ }^{127}$

\footnotetext{
${ }^{126}$ Hierbei handelt es sich um einen Betrag, der auf die vom VfB Stuttgart erhobenen Eintrittspreise aufgeschlagen wird und direkt in die Kassen der Stadt Stuttgart fließt. Für einen Stehplatz beträgt dieser Stadiongroschen 1,- DM, für eine Kurvensitzplatz 1,5 DM und für einen Sitzplatz auf der Haupt- oder Gegentribüne 2,- DM.

${ }^{127}$ Im Fall der Stadt Stuttgart erschiene aufgrund des Ansatzes kalkulatorischer Abschreibungen und Zinsen auch der Begriff Aufwendungen angebracht.
} 
Tabelle 19: Einnahmen, Ausgaben und Kostendeckungsgrad des Stuttgarter Gottlieb-Daimler-Stadions in DM

\begin{tabular}{|c|c|c|c|c|}
\hline \multicolumn{5}{|c|}{ Unterabschnitt 5610, Gottlieb-Daimler-Stadion mit Nebenanlagen } \\
\hline \multicolumn{2}{|l|}{ Jahr } & \multirow{2}{*}{\begin{tabular}{|l|}
1994 Ansatz \\
6.756 .000 \\
\end{tabular}} & \multirow{2}{*}{\begin{tabular}{|l}
1993 Ansatz \\
6.067 .000 \\
\end{tabular}} & \multirow{2}{*}{\begin{tabular}{|l}
$1992 \mathrm{Rg}$. erg. \\
5.484 .989 \\
\end{tabular}} \\
\hline Einnahmen & gesamt & & & \\
\hline \multirow[t]{4}{*}{ darunter: } & $\begin{array}{l}\text { Miete, Pacht aus unbeweglichem } \\
\text { Vermögen }\end{array}$ & 5.613 .000 & 4.967 .100 & 3.136 .008 \\
\hline & $\begin{array}{l}\text { Kostenerstattung von übrigem } \\
\text { Bereich (z.B. für Kassenpersonal) }\end{array}$ & 150.000 & 153.600 & 102.867 \\
\hline & Auflösung von Zuweisungen u.ä. & 976.400 & 779.300 & 301.345 \\
\hline & $\begin{array}{l}\text { Restliche Einnahmepositionen } \\
\text { (zusammengefaßt) }\end{array}$ & 16.600 & 167.000 & 1.944 .769 \\
\hline Ausgaben & gesamt & 15.043 .000 & 14.684 .600 & 11.544 .870 \\
\hline \multirow[t]{13}{*}{ darunter: } & Personalausgaben & 2.773 .700 & 3.086 .100 & 2.830 .489 \\
\hline & $\begin{array}{l}\text { Unterhaltung von städtischen } \\
\text { Gebäuden }\end{array}$ & 980.000 & 1.080 .800 & 1.057 .054 \\
\hline & Unterhaltung von Außenanlagen & 178.000 & 193.000 & 177.921 \\
\hline & Kleine Beschaffungen & 158.000 & 158.000 & 144.785 \\
\hline & $\begin{array}{l}\text { Miete, Pacht für unbewegliches } \\
\text { Vermögen }\end{array}$ & 963.000 & 963.000 & 963.000 \\
\hline & Abgaben für Grundstücke & 264.000 & 264.000 & 259.690 \\
\hline & Heizung, Energiekosten etc. & 644.000 & 676.000 & 614.436 \\
\hline & Reinigungskosten & 135.000 & 145.000 & 123.707 \\
\hline & Betriebsbedarf & 330.500 & 296.700 & 346.126 \\
\hline & Abschreibungen & 4.458 .900 & 3.508 .600 & 2.567 .065 \\
\hline & Verzinsung des Anlagekapitals & 2.939 .200 & 3.000 .700 & 1.108 .829 \\
\hline & $\begin{array}{l}\text { Sonstiges (Beteiligung VfB an } \\
\text { Werbeeinnahmen) }\end{array}$ & 850.000 & 950.000 & 995.664 \\
\hline & $\begin{array}{l}\text { Restliche Ausgabepositionen } \\
\text { (zusammengefaßt) }\end{array}$ & 368.700 & 362.700 & 356.104 \\
\hline \multicolumn{2}{|c|}{ Gesamteinnahmen } & 6.756 .600 & 6.067 .000 & 5.484 .989 \\
\hline \multicolumn{2}{|c|}{ Gesamtausgaben } & 15.043 .000 & 14.684 .600 & 11.544 .870 \\
\hline \multicolumn{2}{|c|}{ Zuschußbedarf } & 8.286 .400 & 8.617 .600 & 6.059 .882 \\
\hline \multicolumn{2}{|c|}{ Kostendeckung in \% } & 44,9 & 41,3 & 47,5 \\
\hline
\end{tabular}

Quelle: Haushaltsplan der Stadt Stuttgart 1993/94, S. 204/205.

Auf der Einnahmeseite ragt die Position "Miete und Pacht aus unbeweglichem Vermögen" deutlich hervor. Hierin enthalten sind die Einnahmen aus Vermietung an den 
VfB. Die deutliche Erhöhung in den Ansätzen 1993 und 1994 gegenüber dem Rechnungsergebnis 1992 beruht größtenteils auf der Erhebung des oben genannten Stadiongroschens, zusätzlicher Gebäudewerbung und auch zusätzlichen Großveranstaltungen. ${ }^{128}$

Auf der Ausgabeseite sind insbesondere die Personalausgaben sowie die Aufwendungen für Abschreibungen und Verzinsung des Anlagekapitals unter der Fülle von Positionen hervorzuheben. Abschreibungen und Verzinsung des Anlagekapitals allein machen dabei mit DM 7,4 MIO im Ansatz für 1994 etwa die Hälfte der Gesamtausgaben aus. ${ }^{129}$

Faßt man die gesamten Einnahmen und Ausgaben schließlich zusammen und setzt sie ins Verhältnis zueinander, dann ergibt sich ein Kostendeckungsgrad von etwa $45 \%$, der angesetzte absolute Zuschußbedarf beträgt für die Haushaltsjahre 1993 und 1994 über acht Millionen DM.

Wenngleich an dieser Stelle nochmals daraufhingewiesen werden soll, daß dieser Haushaltsposten auch noch drei weitere Sportstätten beinhaltet, kann doch zusammenfassend festgehalten werden, daß der Profifußball auch in Stuttgart mit großem finanziellem Aufwand aus dem kommunalen Sportetat gefördert wird.

\section{d) Das Mönchengladbacher Bökelbergstadion}

Neben diesen drei baden-württembergischen Bundesligisten soll nun des weiteren eine nicht-badenwürttembergische Bundesligastadt in die Betrachtung miteinbezogen werden.

Hierzu wurde die Stadt Mönchengladbach ausgewählt, da sich diese Kommune nach Ansicht des Autors am besten dazu eignet, fundierte Aussagen über die Förderung des Profifußballs abzuleiten. Folgende Aspekte mögen diese Behauptung belegen.

-Im Gegensatz zu Freiburg hat die Stadt Mönchengladbach für das städtische Stadion einen eigenen Unterabschnitt 561, "Fußballstadion Bökelberg", eingerichtet. ${ }^{130}$

\footnotetext{
${ }^{128} \mathrm{Vgl}$. hierzu Erläuterungen zum Unterabschnitt 5610, Haushaltsplan der Stadt Stuttgart, 1993/94, S. 205. Legt man bspw. den Zuschauerschnitt des VfB Stuttgart aus den letzten 5 Jahren zugrunde, der sich auf etwa 27.000 Zuschauer pro Spiel belief, die Zahl stammt aus dem Fußballmagazin Kicker, dann dürtten aus dem Stadiongroschen pro Spiel Einnahmen zwischen DM 30.000 bis DM 40.000 zu erwarten sein. Auf eine ganze Saison mit 17 Heimspielen hochgerechnet, eventuell zusätzliche Spiele in nationalen oder internationalen Pokalwettbewerben noch nicht berücksichtigt, könnte dann allein daraus mit Einnahmen in Höhe von DM 500.000 bis DM 800.000 zu rechnen sein.

${ }^{129}$ Dieser hohe Anteil kalkulatorischer Aufwendungen macht nochmals deutlich, daß die Behauptung, der Kostendeckungsgrad des Karlsruher Wildparkstadions würde unter Berücksichtigung derartiger Aufwendungen beträchtlich sinken, keineswegs aus der Luft gegriffen ist. Vgl. Abschnitt 2. 1.6.b.

${ }^{130}$ Dieser Unterabschnitt wurde in Mönchengladbach bereits 1972 eingerichtet und trug bis zur Emeuerung des Gemeindehaushaltsrechts im Jahre 1974 die Gruppierungsziffer 5510.
} 
-Im Gegensatz zu Karlsruhe und insbesondere zu Freiburg kann am Beispiel Mönchengladbach gezeigt werden, wie sich die Förderung eines Bundesligaklubs über einen längeren Zeitraum hin entwickelt hat. ${ }^{131}$

-Im Gegensatz zu den Stadien in Stuttgart und Karlsruhe, die auch anderen Sportarten dienen können, handelt es sich beim Mönchengladbacher Bökelbergstadion um eine reine Fußballarena, so daß der Zuschußbedarf seitens der Kommune ausschließlich als Förderung des Profifußballs angesehen werden kann.

-Im Gegensatz zu Karlsruhe und Freiburg bringt die Stadt Mönchengladbach in ihrem Haushalt auch kalkulatorische Abschreibungen und Zinsen für das betreffende Stadion in Ansatz.

-Im Gegensatz zu allen drei anderen Städten weist der Haushalt der Stadt Mönchengladbach explizit die Einnahmen aus Verkaufsgenehmigungen und Reklameflächen aus, die im Zusammenhang mit den Spielen der ortsansässigen Borussia entstehen.

-Im Gegensatz zu Stuttgart und Karlsruhe handelt es sich beim Mönchengladbacher Bökelbergstadion um eine Sportstätte, in der in jüngster Vergangenheit nicht in diesem großen Stile investiert wurde. Es ist von daher mit vergleichsweise geringeren Kosten, genauer, kalkulatorischen Aufwendungen, zu rechnen.

Es kann folglich festgehalten werden, daß bei diesem Stadion alle Kriterien erfüllt sind, die für eine aussagekräftige Analyse des tatsächlichen Zuschußbedarfs notwendig sind.

Der größte Einnahmeposten im Unterabschnitt 561 des Mönchengladbacher Haushaltsplans sind die Mieten und Pachten. Diese betrugen bis 1992 6\% der Zuschauernettoeinnahmen, seither wird das sogenannte "Catering"132 in vereinseigener Regie durchgeführt, was zu einer Erhöhung der zuschauerabhängigen Miete auf $10 \%$ der Nettoeinnahmen geführt hat.

Einnahmen in einer annähernd gleichen Größenordnung erwirtschaftet die Stadt durch die Vermietung von Reklameflächen, der Ansatz für 1993 sieht hierfür DM 500.000 vor.

Mit der Übertragung des Caterings auf den Verein ging schließlich ein gegenüber den Rechnungsergebnissen der Vergangenheit reduzierter Ansatz der Einnahmen aus Verkaufsgenehmigungen einher. Betrugen diese laut Rechnungsergebnis 1991 noch etwa DM 137.000, so wurden sie für 1993 nur noch mit DM 70.000 angesetzt.

${ }^{131}$ Der Karlsruher SC gehört der ersten Bundesliga ununterbrochen erst seit der Saison 1987/88 an, der SC Freiburg ist erstmals 1993 in diese höchste deutsche Fußballklasse aufgestiegen. Der VFL Mönchengladbach hingegen ist seit 1965 durchgängig Bundesligist.

${ }^{132}$ Der Begriff Catering beinhaltet Einnahmen aus Bewirtung u.ä. 


\section{Abbildung 7: Prozentuale Anteile verschiedener Ausgabepositionen an den Ge- samtausgaben für das Mönchengladbacher Bökelbergstadion im Haushaltsansatz 1993}

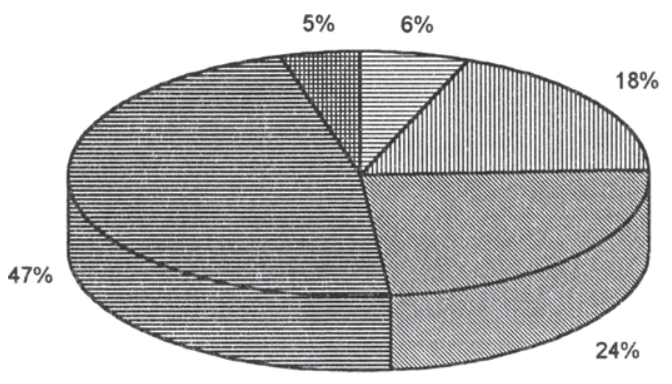

Personalausgaben

Innere Verrechnungen

$\mathbb{Q}$ Kalkulatorische

Abschreibungen

目Kalkulatorische Zinsen

田 Sonstiges

Quelle: Haushaltsplan der Stadt Mónchengladbach 1992/93, S. 338/39.

Einen weiteren, von seiner Größenordnung her bedeutenden Posten stellen die Mehrwertsteuereinnahmen dar, die zum Zwecke der Errechnung eines Kostendeckungsgrades aus kommunaler Sicht allerdings aus dem betreffenden Haushalt herausgerechnet werden müssen. ${ }^{133}$

Auf der Ausgabeseite sind neben den Personalausgaben (DM 221.500) und verschiedenen inneren Verrechnungen (DM 647.700) wie für Versicherungen, Steuern, Energiekosten, Grundstücksunterhaltung, Reinigung, etc. insbesondere die kalkulatorischen Aufwendungen zu erwähnen, deren relativer Anteil an den Gesamtausgaben mit $71 \%$ (vgl. Abbildung 7) den Vergleichswert aus Stuttgart (49\%) sogar noch übersteigt. Absolut gesehen betragen die kalkulatorischen Abschreibungen DM 857.100, kalkulatorische Zinsen wurden im Haushalt 1993 in Höhe von DM 1.682.200 in Ansatz gebracht. ${ }^{134}$

\footnotetext{
${ }^{133}$ Die Umsatzsteuer ist zu $65 \%$ eine Bundes-, zu 35\% eine Ländersteuer und kann damit nicht in die Berechnung des kommunalen Kostendeckungsgrades eingehen.

${ }^{134}$ Dem Ansatz liegen je nach Nutzungsdauer unterschiedliche Abschreibungssätze zugrunde. Die Abschreibung erfolgt vom Wiederbeschaffungszeitwert. Der Zinssatz beträgt 7\%. Berechnungsbasis ist ebenfalls der Wiederbeschaffungszeitwert. Vgl. Haushaltsplan der Stadt Mönchengladbach 1992/93, S. 339.
} 
Tabelle 20: Einnahmen, Ausgaben und Kostendeckungsgrad des Mönchengladbacher Bökelbergstadions in DM

\begin{tabular}{|c|c|c|c|c|}
\hline \multicolumn{5}{|c|}{ Unterabschnitt 561, Fußballstadion Bökelberg } \\
\hline \multicolumn{2}{|l|}{ Jahr } & \multirow{2}{*}{\begin{tabular}{|l|}
1993 Ansatz \\
1.398 .100 \\
\end{tabular}} & \multirow{2}{*}{\begin{tabular}{|l}
1992 Ansatz \\
1.168 .100 \\
\end{tabular}} & \multirow{2}{*}{$\begin{array}{l}1991 \mathrm{Rg} . \text { erg. } \\
1.290 .049 \\
\end{array}$} \\
\hline Einnahmen & gesamt & & & \\
\hline \multirow[t]{6}{*}{ darunter: } & Mieten und Pachten & 590.000 & 280.000 & 405.373 \\
\hline & Verkaufsgenehmigungen & 70.000 & 150.000 & 137.349 \\
\hline & Nebenleistungen aus Mietverträgen & 38.000 & 38.000 & 34.802 \\
\hline & Nebenlstg. aus Reklameflächen & 500.000 & 520.000 & 539.976 \\
\hline & Vermischte Einnahmen & 100 & 100 & - \\
\hline & Mehrwertsteuer & 200.000 & 180.000 & 172.546 \\
\hline Ausgaben & gesamt & 3.572 .100 & 3.085 .600 & 2.524 .971 \\
\hline \multirow[t]{5}{*}{ darunter: } & Personalausgaben & 221.500 & 203.900 & 197.467 \\
\hline & Innere Verrechnungen & 647.700 & 510.800 & 593.794 \\
\hline & Kalkulatorische Abschreibungen & 857.100 & 784.800 & 770.948 \\
\hline & Kalkulatorische Zinsen & 1.682 .200 & 1.562 .600 & 925.583 \\
\hline & Restliche Ausgabepositionen (zus.) & 163.600 & 23.500 & 37.178 \\
\hline \multicolumn{2}{|c|}{ Gesamteinnahmen } & 1.398 .100 & 1.168 .100 & 1.290 .049 \\
\hline \multicolumn{2}{|c|}{ Gesamtausgaben } & 3.572 .100 & 3.085 .600 & 2.524 .971 \\
\hline \multicolumn{2}{|c|}{ Zuschußbedarf } & 2.174 .000 & 1.917 .500 & 1.234 .922 \\
\hline \multicolumn{2}{|c|}{ Kostendeckung in \% } & 39,1 & 37,8 & 51,1 \\
\hline \multicolumn{2}{|c|}{ Kostendeckung ohne Umsatzsteuer in \% } & 33,5 & 32,0 & 44,3 \\
\hline
\end{tabular}

Quelle: Stadt Mönchengladbach, Haushaltsplan der Stadt Mönchengladbach 1992/93, S. 338/39.

Tabelle 20 liefert die Zahlengrundlage für die Errechnung des Kostendeckungsgrades des Mönchengladbacher Bökelbergstadions. Dieser ist mit Werten von 44, 32 und 34 $\%$ in den Jahren 1992, 93 und 94 sogar noch geringer als jener des Stuttgarter GottliebDaimler-Stadions. Eine längerfristige Betrachtung des Haushaltspostens Bökelbergstadion zeigt, daß der prozentuale Kostendeckungsgrad Ende der 70er und Anfang der 80er Jahre höher war. Dies ist in erster Linie auf mittlerweile nominal deutlich höhere Ansätze der kalkulatorischen Aufwendungen sowie nominal stagnierende oder zuweilen gar sinkende Mieteinnahmen zurückzuführen. ${ }^{135}$ Insbesondere der letzte Punkt erscheint interessant, zeigt er doch auf, daß mit dem sportlichen Erfolg der Zuschauerzuspruch variieren kann, damit die Mieteinnahmen steigen bzw. sinken und somit die

${ }^{135} \mathrm{Vgl}$. hierzu Anhang 3. 
Höhe des Kostendeckungsgrades auch vom sportlichen Erfolg einer Mannschaft abhängen kann. ${ }^{136}$

Abschließend zur Betrachtung der kommunalen Profifußballförderung kann festgehalten werden, daß alle betrachteten Städte ihre Bundesligaclubs netto im Rahmen ihrer Sportetats unterstützen.

Die Kostendeckungsgrade der jeweiligen Stadien liegen in allen Fällen, in denen eine Ermittlung möglich war, unter $50 \%$. Der absolute Zuschußbedarf überschreitet in Mönchengladbach die 2-Millionen-Grenze, in Stuttgart die 8-Millionen-Grenze und auch der absolute Zuschußbedarf in Karlsruhe läge - würden kalkulatorische Aufwendungen in Ansatz gebracht - weit über dem Mönchengladbacher Wert.

Integriert man diese indirekte öffentliche Förderung in die Etats der jeweiligen Vereine, dann zeigt sich, daß die kommunale Netto-Infrastrukturleistung einen beträchtlichen Teil der "bereinigten" Gesamtbudgets von Fußballbundesligaclubs ausmachen kann. So beträgt der Anteil des kommunalen Zuschußbedarfs an dem um diesen $\mathrm{Zu}$ schußbedarf aufgestockten Etat des VfB Stuttgart immerhin knapp 30\%. ${ }^{137}$ Dieser Prozentsatz ist in jenen Städten, in denen in jüngster Vergangenheit nicht in diesem Ausmaß in die jeweilige Fußballarena investiert wurde, selbstverständlich geringer.

${ }^{136}$ Dieses Phänomen einer positiven Korrelation zwischen sportlichem Erfolg und Kostendeckungsgrad kann allerdings keineswegs verallgemeinert werden. So gibt es einige Vereine, deren Zuschauerzahlen keineswegs so stark mit dem sportlichen Erfolg der Mannschaft korrelieren. Zu derartigen Vereinen gehören bspw. Schalke 04 oder der 1. FC Kaiserslautern.

${ }^{137}$ Dieser Berechnung liegt eine Angabe der WirtschaftsWoche zugrunde, wonach sich der Etat des VfB Stuttgart in der Saison 1994/95 auf DM 20 Millionen beläuft. Vgl. WirtschaftsWoche Nr. 34 vom 19.08.1994, S. 37. 


\subsubsection{Quantitative Aspekte kommunaler Sportförderung}

\subsubsection{Die Entwicklung kommunaler Sportausgaben von 1965 - 1991}

Nachdem mit Ausnahme des Profifußballs bislang eher qualitative Aspekte kommunaler Sportförderung aufgezeigt wurden, werden sich die beiden folgenden Abschnitte primär quantitativen Gesichtspunkten kommunaler Sportförderung zuwenden. $\mathrm{Zu}$ deren langfristiger Darstellung und Analyse ${ }^{138}$ wurde auf die Reihe "Finanzen und Steuern" des Statistischen Bundesamtes zurückgegriffen, welche die Rechnungsergebnisse öffentlicher Haushalte in bezug auf den Sport jährlich in Fachserie 14, Reihe 3.5., "Rechnungs-ergebnisse der öffentlichen Hauhalte für Gesundheit, Sport und Erholung", dokumentiert. ${ }^{139}$

Betrachtet wurden die Ausgaben für Förderung des Sports, Sportstätten und Badeanstalten, eine Aufteilung der Sportfördermittel nach verschiedenen Sportarten erwies sich als nicht möglich. Der Versuch, derartige Gliederungen zumindest exemplarisch für einige Kommunen von den jeweiligen Sportämtern zu erhalten, schlug fehl. Lediglich für eine Stadt konnten, allerdings nicht über einen längeren Zeitraum hinweg, nach verschiedenen Vereinen differenzierende Daten gewonnen werden. ${ }^{140}$

Auch die Erfassung der haushaltsanalog gegliederten Sportausgaben durch Betrachtung der Veröffentlichungen des Statistischen Bundesamtes stellte den Untersucher vor einige Probleme. So zeigte die Analyse der Bände seit 1954 einige Strukturbrüche bei der Unterteilung der ausgewiesenen Daten, weshalb die Sportausgaben letztlich auch erst ab 1965 dargestellt werden. ${ }^{141}$ Weitere Änderungen in der Darstellungsweise des Statistischen Bundesamtes nach 1965 konnten eigenhändig bereinigt werden, was letztlich die Darstellung vergleichbarer Zahlenwerte über den oben genannten Zeitraum hinweg ermöglichte. ${ }^{142}$

${ }^{138}$ Die folgenden Ausfuhrungen zu den quantitativen Aspekten öffentlicher Sportförderung sind rein deskriptiver Natur. Eine Erörterung unter Berücksichtigung allokationstheoretischer Überlegungen erfolgt innerhalb der Kapitel IV und V.

${ }^{139}$ Die Dokumentation der Sportausgaben findet sich erst seit 1980 in der o.g. Fachserie 14, Reihe 3.5. Vor 1980 wurden die Sportausgaben in einigen anderen Reihen dokumentiert, welche in den Anhängen 4 bis 7 jeweils in den Quellenangaben aufgelistet sind.

${ }^{140}$ Es handelt sich hierbei um die Stadt Tuttlingen, welche die personellen Fördergelder getrennt nach verschiedenen Vereinen auflistet, eine konkrete Aussage zu einem möglicherweise unterschiedlichen quantitativen Ausmaß der Sportausgaben in Zusammenhang mit verschiedenen Sportarten ist aber auch damit kaum möglich, da sogenannte Mehrspartenvereine existieren, d.h. Vereine, die verschiedene Sportarten anbieten.

${ }^{141}$ In den Jahren vor 1963 beinhalten die Sportausgaben auch die Ausgaben für die Jugendpflege.

${ }^{142}$ Das Statistische Bundesamt weist die Unterteilung in laufende und einmalige Ausgaben erst ab dem Erscheinungsjahr 1984 explizit aus. Für die Haushaltsjahre 1970 bis 1983 wurden die laufenden und investiven Anteile eigenhändig rückgerechnet. Vgl. hierzu die Erläuterungen innerhalb von Anhang 4, 
Zunächst zur Aufteilung der gesamten nominalen Sportausgaben (Förderung des Sports, Sportstätten und Badeanstalten) nach verschiedenen Ausgabe- und Einnahmearten, welche in Abbildung 8 dargestellt wird.

\section{Abbildung 8: Kommunale Sportausgaben von 1965 bis 1991 in MIO DM, nomi- nal, getrennt nach verschiedenen Ausgabe- und Einnahmearten}

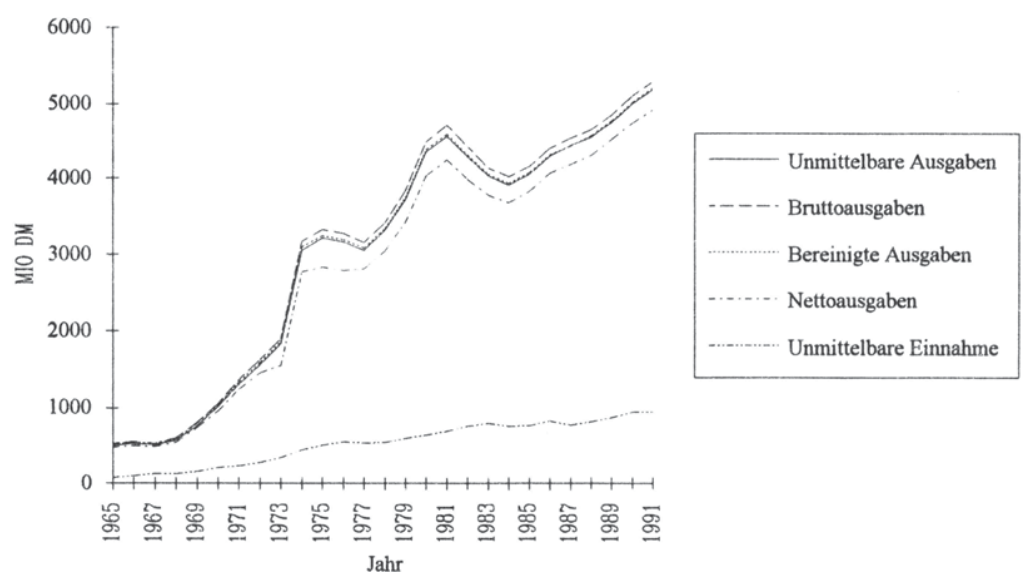

Quelle: Statistisches Bundesamt, Finanzen und Steuern, verschiedene Reihen, Jahrgånge 1965 bis 1991.

Es zeigt sich im Trend ein Anstieg der nominalen Sportausgaben über den betrachteten Zeitraum hinweg, der nominal gesehen bis 1991 anhält. Mitte der 70er und Anfang der 80er Jahre sind allerdings Abweichungen von diesem Trend festzustellen.

Beide Ausreißer können sowohl als Abweichungen nach oben wie auch nach unten gedeutet werden. Insbesondere im ersten Fall liegt die erstgenannte Interpretation nahe, zeigt sich doch im Jahr 1974 ein gegenüber den Vorjahren vergleichsweise starker Anstieg der Sportausgaben.

Der zweite Ausreißer hingegen legt aufgrund der zu beobachtenden, mehrere Jahre anhaltenden Reduzierung der Ausgaben eine Interpretation als Abweichung nach unten nahe.

Seite 3. Für die Jahre 1965 bis 1969 konnten die laufenden und investiven Anteile anhand der Fachserie L, Reihe 1, "Haushaltswirtschaft von Bund, Ländern und Gemeinden, II. Jahresabschlüsse, Kommunale Finanzen" ermittelt werden. Vgl. ebd. 
Dieser Trend wie auch die beiden beobachteten Ausreißer sollen nunmehr Schritt für Schritt lokalisiert und an späterer Stelle auch erklärt werden.

Das Gesamtausmaß der Ausgaben betrug bspw. im Jahr 1965 brutto etwa DM 0,53 MRD, netto verausgabten die Kommunen im selben Jahr DM 487 MIO für den Sport. Zum Vergleich, im Jahr 1991 belief sich das Gesamtausmaß brutto auf etwa DM 5,3 MRD und damit nominal auf das Zehnfache. Netto waren es im selben Jahr DM 4,935 MRD, die die Kommunen der alten Bundesländer für den Sport verausgabten. Der letztlich resultierende kommunale Zuschußbedarf belief sich 1991 auf DM 3,986 MRD. ${ }^{143}$

\section{Abbildung 9: Kommunale Nettosportausgaben von 1965 bis 1991 in MIO DM, no- minal, getrennt nach verschiedenen Haushaltsabschnitten}

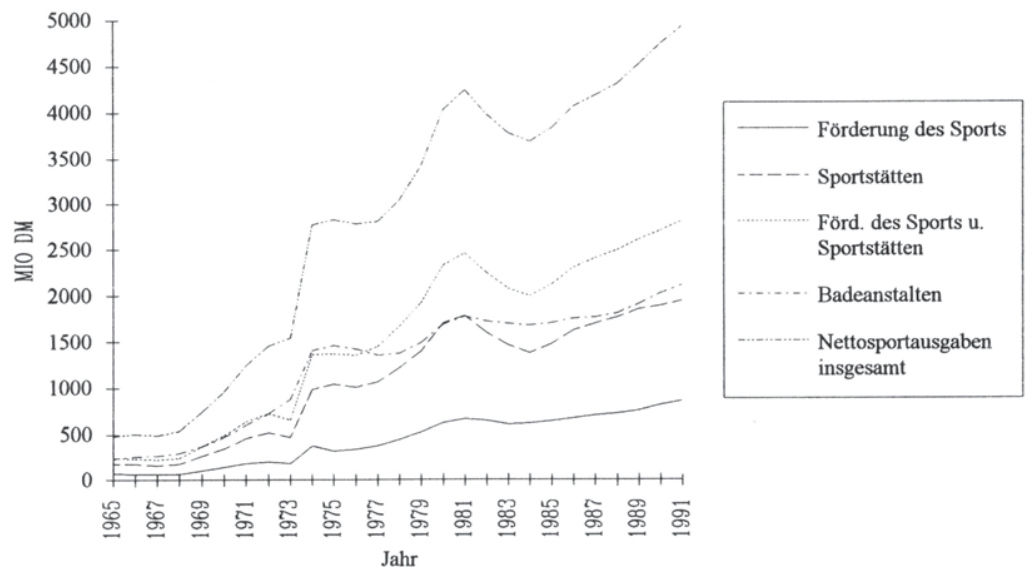

Quelle: Statistisches Bundesamt, Finanzen und Steuern, verschiedene Reihen, Jahrgänge 1965 bis 1991.

\footnotetext{
${ }^{143}$ Die Bruttoausgaben errechnen sich durch Hinzunahme der Zahlungen an den öffentlichen Bereich zu den Unmittelbaren Ausgaben. Zieht man von diesen Bruttoausgaben die Zahlungen von gleicher Ebene, d.h. von anderen Kommunen ab, dann erhält man die Bereinigten Ausgaben. Aus diesen lassen sich, verringert um die Zahlungen von anderen Gebietskörperschaften, die Nettoausgaben der Kommunen errechnen. Zieht man hiervon schließlich die Unmittelbaren Einnahmen ab, dann erhält man den $\mathrm{Zu}$ schußbedarf und damit die tatsächliche finanzielle Nettobelastung der Kommunen. Vgl. hierzu die oben genannten Veröffentlichungen des Statistisches Bundesamtes oder auch die Erläuterungen zu den Anhängen 4 bis 7 .
} 
Ein ähnliches Bild liefert Abbildung 9, welche die Nettosportausgaben nach den verschiedenen Haushaltsabschnitten differenziert. Der Großteil der Sportausgaben wird, zumindest seit Mitte der 70er Jahre, für die Förderung des Sports und Sportstätten ausgegeben; 1991 beliefen sich die Nettoausgaben dieser beiden Haushaltsabschnitte auf DM 2,815 MRD. ${ }^{144}$ Bau und Unterhaltung von Badeanstalten ließen sich die Kommunen im selben Jahr etwa DM 2,1 MRD kosten.

Betrachtet man nochmals die beiden Ausreißer Mitte der 70er sowie Anfang der 80er Jahre, dann zeigt sich folgendes Bild:

Die starke Erhöhung der Gesamtausgaben im Jahr 1974 ist sowohl bei den Haushaltsabschnitten Förderung des Sports und Sportstätten, als auch bei den Badeanstalten zu beobachten. Es kann folglich nicht die Fußballweltmeisterschaft 1974 allein gewesen sein, die den Umfang kommunaler Sportförderung auf dieses bis dahin noch nicht gekannte Niveau hat ansteigen lassen.

Abbildung 9 zeigt ebenfalls, daß beide Ausgabetypen in den Jahren 1975 und 1976 keine weiteren Zuwächse erfuhren, diese annäherungsweise Stagnation bei den Badeanstalten gar bis einschließlich 1978 anhielt.

Im zweiten Fall (1982 bis 1984) läßt sich der Ausreißer in erster Linie auf die Ausgaben für Förderung des Sports und für Sportstätten reduzieren.

\footnotetext{
${ }^{144}$ Eine explizite Trennung zwischen den Ausgaben für Förderung des Sports und Sportstätten erfolgt erst ab dem Jahr 1974. Für die Haushaltsjahre bis 1973 wurden die jeweiligen Anteile eigenhändig rückgerechnet. Vgl. hierzu die Erläuterungen innerhalb von Anhang 4, Seite 3.
} 
Nun erfordert eine Langzeitbetrachtung eine Berücksichtigung der Preisentwicklung und damit eine Deflationierung der Werte, da nur anhand inflationsbereinigter Zahlen korrekte Aussagen über tatsächliche absolute Trends getroffen werden können. Abbildung 10 veranschaulicht die Entwicklung der gesamten Nettosportausgaben nominal und real, als Basisjahr zur Preisbereinigung wurde das Jahr 1985 gewählt. ${ }^{145}$

Der Vergleich nominaler und realer kommunaler Sportausgaben belegt, daß die weiter oben getroffene Feststellung eines langfristigen Trends ansteigender Sportausgaben zum heutigen Zeitpunkt nicht widerspruchsfrei aufrechterhalten werden kann. Die kommunalen Sportausgaben stiegen real bis 1980 an, damit war allerdings das höchste Niveau erreicht. Zwar läßt sich seit 1984 wieder ein stetiger realer Anstieg der Sportausgaben feststellen, der Stand von 1980 mit real DM 4,873 MRD wurde jedoch in den Folgejahren nicht mehr erreicht.

\section{Abbildung 10: Kommunale Nettosportausgaben von 1965 bis 1991 in MIO DM, nominal und real, Basisjahr 1985}

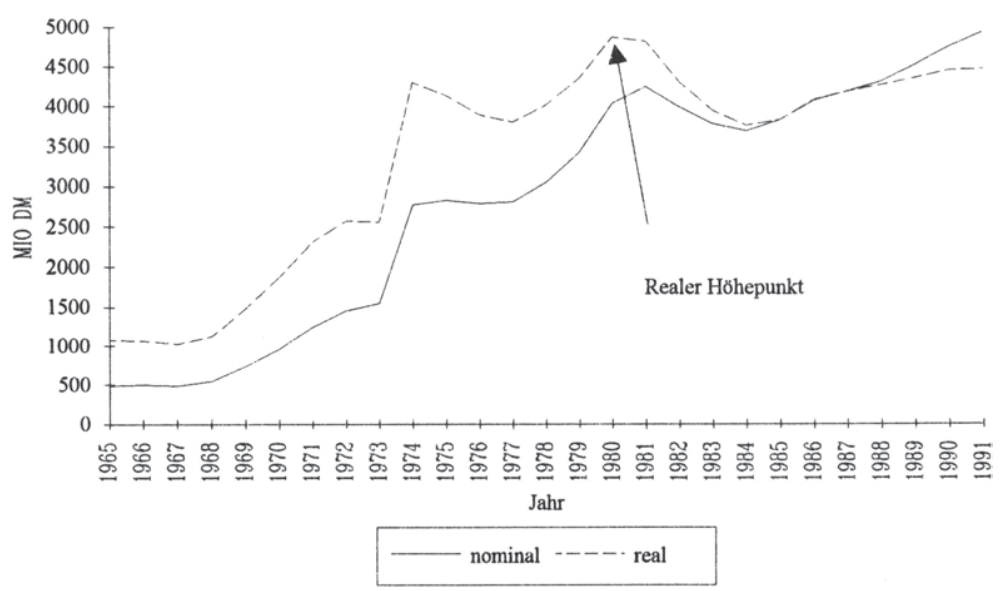

Quelle: Statistisches Bundesamt, Finanzen und Steuern, verschiedene Reihen, Jahrgănge 1965 bis 1991.

${ }^{145}$ Als Grundlage für die Deflationierung der Werte diente ein vom Statistischen Bundesant angewandter Gesamt-Lebenshaltungs-Preisindex. Vgl. Statistisches Bundesamt, Statistische Jahrbücher, 1966 1992. 
Festzustellen ist überdies, daß die beiden erklärungsbedürftigen Ausreißer auch real auftreten, folglich also nicht auf Preisniveauveränderungen zurückgeführt werden können.

Einen weiteren Ansatz, Abweichungen vom Trend näher zu lokalisieren, bietet möglicherweise die Unterteilung der Gesamtausgaben in laufende und einmalige Bestandteile. Ein Blick auf Abbildung 11 belegt den vermuteten Tatbestand. Die beiden Trendabweichungen beruhen im wesentlichen auf verändertem Investitionsverhalten der Kommunen, während sich die laufenden Ausgaben nominal durch einen recht stetigen Anstieg auszeichnen.

\section{Abbildung 11: Laufende und investive nominale kommunale Sportausgaben in MIO DM von 1965 bis $1991^{146}$}

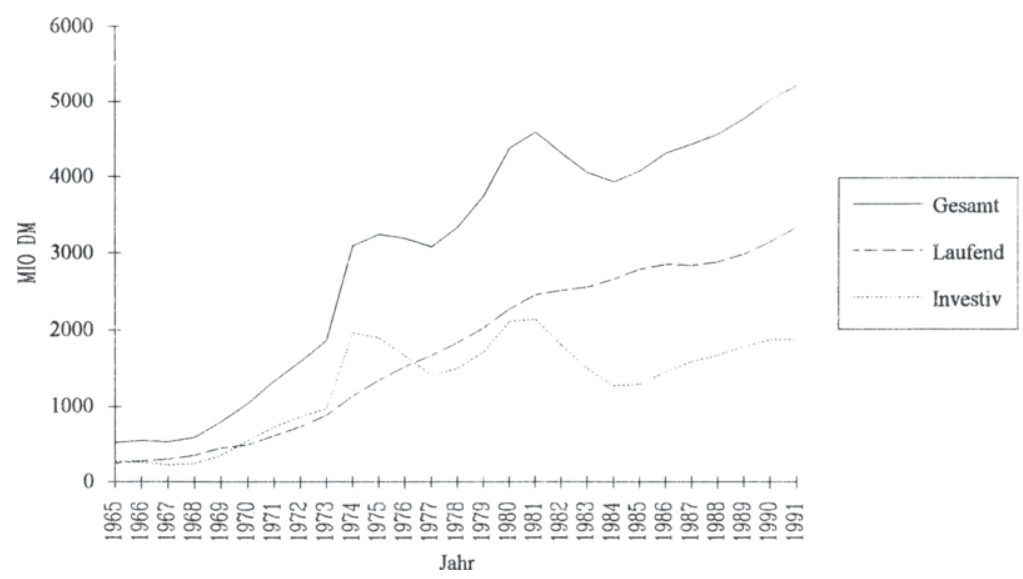

Quelle: Statistisches Bundesamt, Finanzen und Steuern, verschiedene Reihen, Jahrgånge 1965 bis 1991.

Ähnliches verdeutlicht auch ein Blick auf die Ausgabeentwicklung einzelner Kommunen. In sämtlichen Fällen zeichnet sich die Entwicklung der laufenden Ausgaben durch einen mehr oder weniger stetigen Anstieg aus, während das Investitionsvolumen in den verschiedenen Perioden durchaus variiert. Im Gegensatz zur aggregierten Betrachtung

\footnotetext{
${ }^{146}$ Die Aufteilung in investive und laufende Anteile orientiert sich an den Bereinigten Ausgaben, vgl. auch Anhang 4.
} 
konnten auf einzelwirtschaftlicher Ebene hinsichtlich der laufenden Ausgaben allerdings einzelne Ausgabesprünge festgestellt werden, die mit den einsetzenden Unterhaltungs- bzw. Folgekosten nach Abschluß größerer Baumaßnahmen zusammenhängen. ${ }^{147}$

Trennt man die laufenden und einmaligen Ausgaben ferner auf die beiden Ausgabearten Sportstätten und Förderung des Sports einerseits sowie Badeanstalten andererseits auf, dann zeigt sich folgendes, in Abbildung 12 dargestelltes Bild. Beide Trendabweichungen sind in erster Linie auf Veränderungen im Investitionsverhalten zurückführen. Abbildung 12 verdeutlicht darüber hinaus, da $\beta$ das höchste Niveau kommunaler Investitionen in Badeanstalten real bereits 1974 erreicht wurde. Betrug der investive Anteil an den Gesamtausgaben für Badeanstalten im Jahr 1974 noch fast $60 \%$, so lag er im Jahr 1985 bei etwa 15, im Jahr 1991 bei knapp 25\%.

\section{Abbildung 12: Laufende und einmalige reale Ausgaben für Förderung des Sports und Sportstätten sowie Badeanstalten von 1965 bis 1991 in MIO DM, Basisjahr 1985}

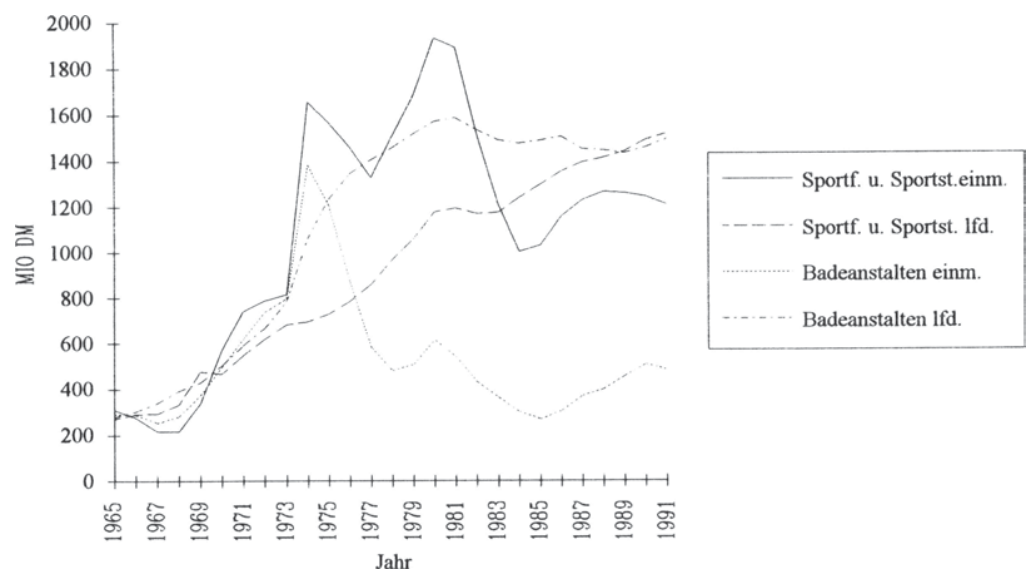

Statistisches Bundesamt, Finanzen und Steuern, verschiedene Reihen, Jahrgänge 1965 bis 1991.

${ }^{147} \mathrm{Vgl}$. unter anderem die Haushaltspläne der Städte Freiburg, Karlsruhe, Lahr, Mönchengladbach, Offenburg und Wolfsburg, 
Ähnliche Zusammenhänge lassen sich bei den Ausgaben für Sportförderung und Sportstätten verzeichnen. Im Jahr des höchsten relativen Anteils der Investitionen an den gesamten Ausgaben, 1980, wurden fast $70 \%$ der öffentlichen Mittel für Investitionen verwandt, 1991 waren es noch knapp $45 \%$.

Es kann damit festgehalten werden, daß die Schwankungen der Sportausgaben im Betrachtungszeitraum vorrangig auf Schwankungen der Investitionen beruhen. Die laufenden Sportausgaben hingegen zeichnen sich durch eine weitaus höhere Kontinuität aus. Es wird Aufgabe von Kapitel V sein, die Betsimmungsgründe insbesondere dieser im Zeitablauf beträchtlich schwankenden Sportinvestitionen näher zu beleuchten.

Eine im Zusammenhang mit der Frage nach der tatsächlichen finanziellen Nettobelastung der Kommunen relevante Größe sind schließlich die Benutzungsentgelte als Bestandteil der Unmittelbaren Einnahmen. Selbige fallen im Zusammenhang mit der Förderung des Sports vorrangig durch laufende Zuschüsse von anderen Bereichen an, welche vom Statistischen Bundesamt allerdings seit 1984 nicht mehr explizit ausgewiesen werden. ${ }^{148}$ Im Haushaltsunterabschnitt Sportstätten bestehen die Unmittelbaren Einnahmen primär aus den oben genannten Gebühren und Entgelten, Einnahmen aus wirtschaftlicher Tätigkeit wie Mieten und Pachten und einigen weiteren, nicht näher spezifizierten übrigen Einnahmetypen. Bei den Badeanstalten sind es fast ausschließlich die Gebühren und sonstigen Entgelte, die auf der Einnahmeseite als Unmittelbare Einnahmen verbucht werden.

Der Vergleich zwischen Unmittelbaren Einnahmen und Nettosportausgaben ermöglicht den Ausweis des prozentualen Zuschußbedarfs der kommunalen Bereitstellung von Sportstätten einerseits und Badeanstalten andererseits und quantifiziert somit den Kostendeckungsgrad der öffentlichen Bereitstellung derartiger Sportanlagen.

Dieser prozentuale Zuschußbedarf oder auch der Kostendeckungsgrad als sein Komplement wird insbesondere im Rahmen von Kapitel IV von Bedeutung sein, wenn innerhalb der allokationstheoretischen Erörterungen zur öffentlichen Sportförderung die Frage in den Raum gestellt wird, ob sich das finanzielle Niveau staatlicher Ausgaben mit der Theorie der öffentlichen Güter erklären läßt, oder ob es alternativer theoretischer Ansätze bedarf, um das grundsätzliche Auftreten und auch das finanzielle Ausmaß dieses Typs staatlicher Subventionen nachvollziehen zu können.

\footnotetext{
${ }^{148} \mathrm{Im}$ Gegensatz zu den Zuschüssen anderer Gebietskörperschaften handelt es sich bei den laufenden Zuschüssen von anderen Bereichen um Erstattungen von privaten Unternehmungen, Spenden etc. $\mathrm{Vgl}$. unter anderem Haushaltspläne der Städte Karlsruhe und Wolfsburg.
} 


\section{Abbildung 13: Prozentualer Zuschußbedarf bei Ausgaben für Förderung des Sports und Sportstätten sowie Badeanstalten von 1965 bis 1991}

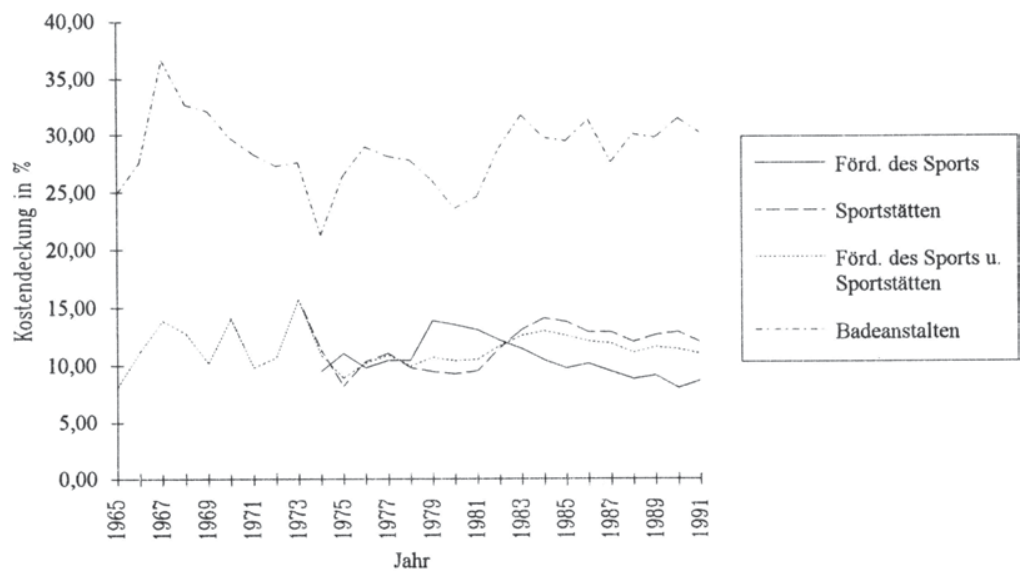

Quelle: Statistisches Bundesamt, Finanzen und Steuern, verschiedene Reihen, Jahrgänge 1965 bis 1991.

Abbildung 13 belegt, daß lediglich ein geringer Anteil der von den Kommunen verausgabten Gelder über Unmittelbare Einnahmen erwirtschaftet wird. Bei den Badeanstalten beträgt der Kostendeckungsgrad im Durchschnitt der betrachteten Jahre knapp $30 \%$. Dies bedeutet de facto, daß die Nutzer kommunaler Badeanstalten nur zu knapp $30 \%$ unmittelbar an den entstehenden Kosten der Bereitstellung dieser Einrichtungen beteiligt werden. Die restlichen $70 \%$ der insgesamt zu deckenden Kosten werden von der Allgemeinheit getragen. ${ }^{149}$

Gleiches gilt in noch viel stärkerem Maße für die Bereitstellung kommunaler Sportstätten. Der Kostendeckungsgrad der Bereitstellung von kommunalen Sportplätzen und hallen beträgt im Durchschnitt der betrachteten Jahre knapp 12\%, was wiederum einer 88\%igen Finanzierung dieser Anlagen durch die Allgemeinheit entspricht. Zum Vergleich, die Kostendeckungsgrade bei den beiden Fußballbundesligastadien, für die eine derartige Angabe möglich erschien, beliefen sich auf etwa $40 \% .{ }^{150}$ Bei den kommuna-

${ }^{149}$ Einschränkend muß selbstverständlich festgehalten werden, daß die Benutzer selbst wiederum der Allgemeinheit angehören und der letztlich von ihnen getragene Anteil an den gesamten Kosten damit die o.g. $30 \%$ etwas übersteigen dürfte.

${ }^{150}$ Es waren dies das Stuttgarter Gottlieb-Daimler- und das Mönchengladbacher Bökelbergstadion. 
len Ausgaben für die Förderung des Sports bewegt sich das Verhältnis von Unmittelbaren Einnahmen zu Nettoausgaben auf ähnlichem Niveau.

Abbildung 14 zeigt abschließend zur Betrachtung der Sportausgaben im Zeitablauf die relative Bedeutung der drei verschiedenen Haushaltsabschnitte.

\section{Abbildung 14: Prozentuale Anteile verschiedener Sportmaßnahmen am Gesamt- Sport-Haushalt 1991, Bereinigte Ausgaben}

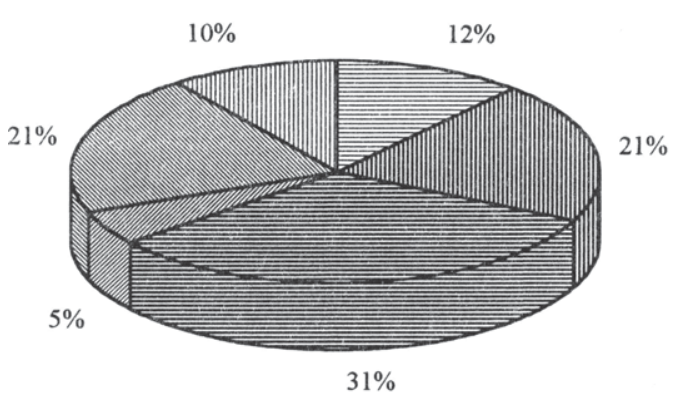

日Förderung des Sports, laufende Rechnung

m Sportstätten, laufende

Rechnung

目Badeanstalten, laufende

Rechnung

ש Förderung des Sports, Kapitalrechnung

$\mathbb{B}$ Sportstätten, Kapitalrechnung

m Badeanstalten,

Kapitalrechnung

Quelle: Statistisches Bundesamt, Finanzen und Steuern, 1991.

Zusammenfassend kann festgehalten werden:

Die Ausgaben der Kommunen für den Sport sind in den vergangenen 27 Jahren stark angestiegen, selbst deflationiert belief sich der Anstieg auf etwa $400 \%$. Der reale Höchstpunkt kommunaler Sportausgaben wurde 1980 erreicht, was in erster Linie auf starke Reduzierungen bei den investiven Maßnahmen zurückzuführen ist. Ebenfalls die einmaligen Ausgaben sind es, die für die beiden beobachteten Trendabweichungen verantwortlich zeichnen. Es wird an späterer Stelle Aufgabe der Untersuchung sein, den starken Anstieg wie auch die Trendausreißer näher zu beleuchten um damit Aussagen über die Bestimmungsfaktoren des Gesamtniveaus kommunaler Sportausgaben treffen zu können, welche letztlich in Kapitel V diskutiert werden.

Daneben konnten die Beobachtungen in bezug auf die Frage nach der fiskalischen Äquivalenz nachweisen, daß der Großteil der Finanzierungslast zur Bereitstellung kommunaler Sportstätten und Badeanlagen nicht durch Unmittelbare Einnahmen in 
Form von Gebühren und Entgelten durch die jeweiligen Nutzer, sondern durch die Allgemeinheit getragen wird. Es wird aus diesem Grunde ebenfalls Aufgabe der theoretischen Überlegungen sein, die dargestellte Gewährung öffentlicher Subventionen zu hinterfragen.

\subsubsection{Die Sportausgaben der Kommunen im Jahr 1991 im Querschnitt}

Um neben der Langzeit- auch eine Querschnittsanalyse kommunaler Sportausgaben zum Zwecke eines interkommunalen Vergleichs durchführen zu können, wurde eine Erhebung der gemeindlichen Sportausgaben des Rechnungsjahres 1991 durchgeführt, deren Ergebnisse in den folgenden Ausführungen skizziert werden soll. ${ }^{151}$

Mit Ausnahme der Stadtstaaten Berlin, Hamburg und Bremen wurden die Sportausgaben sämtlicher 521 deutscher Kommunen in einer Größenordnung von über 20.000 Einwohnern abgefragt. In Analogie zur Aufteilung der Sportausgaben in den Kommunalhaushalten sah die Abfrage eine Differenzierung der Ausgaben nach den drei Haushaltsabschnitten 550, 560 und 570, jeweils getrennt nach einigen weiteren Untergliederungen sowie nach dem Verwaltungs- und Vermögenshaushalt vor. Von den 518 in Zusammenarbeit mit dem Deutschen Städtetag angeschriebenen Kommunen antworteten 503 auf die verschickten Erhebungsbögen, was einer Rücklaufquote von 97,1\% entspricht. Neben einer im Vergleich zu den Angaben des Statistischen Bundesamtes etwas tiefer gegliederten Aufteilung der gesamten Sportausgaben auf verschiedene Sportbereiche, konnte anhand dieser Erhebung insbesondere ein Vergleich der Sportausgaben der einzelnen Kommunen untereinander erfolgen.

${ }^{151} \mathrm{Vgl}$. Hockenjos, 1992, S. $278-305$. 


\section{Abbildung 15: Prozentuale Anteile verschiedener Sportmaßnahmen am Gesamt- Sport-Haushalt 1991, Bruttoausgaben}

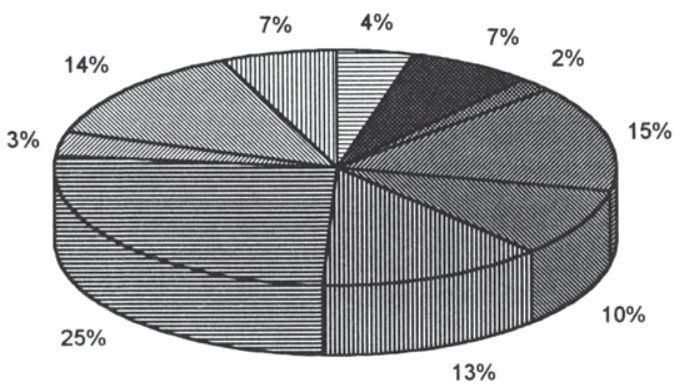

\begin{tabular}{|c|}
\hline 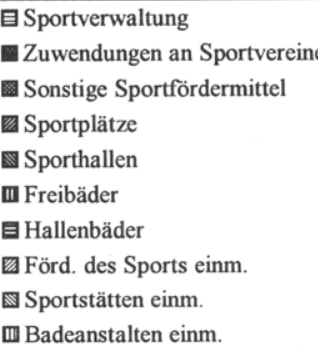 \\
\hline
\end{tabular}

Abbildung 15 betrachtet zunächst die prozentualen Anteile verschiedener Sportgelder am Gesamtetat der Kommunen. Ähnlich wie in Abbildung 13 zeigt sich die Dominanz der Ausgaben für Sportstätten und Badeanstalten, wobei Abbildung 15 darüber hinaus belegt, daß der Großteil der Sportstättengelder für Sportplätze, jener der Aufwendungen für Badeanstalten im Zusammenhang mit Hallenbädern verausgabt wird. ${ }^{152}$ Ausgaben für die Fördenung des Sports entstehen in erster Linie durch die Gewährung direkter Zuwendungen an Sportvereine.

Wendet man sich der reinen Querschnittsanalyse zu, dann ergibt sich folgendes Bild: Die einzelnen Kommunen unterscheiden sich sowohl hinsichtlich der Aufteilung ihrer Sportmittel auf einmalige und laufende Maßnahmen als auch in bezug auf das absolute Niveau ihrer Sportausgaben in erheblichem Maße.

Zunächst zur Trennung in laufende und einmalige Ausgaben. Durchschnittlich verausgabten die betrachteten 503 Kommunen im Rechnungsjahr 1991 77,4\% ihrer Sportgelder für laufende Zwecke. Betrachtet man jedoch die Kommunen im einzelnen, dann zeigen sich Unterschiede von weniger als 20 bis hin zu einem 100\%igen Anteil der

\footnotetext{
${ }^{152}$ Der innerhalb von Abbildung 16 im Vergleich zu Abbildung 14 festzustellende geringere Anteil einmaliger an den gesamten Ausgaben kann zum einen aus den verschiedenen Berechnungsgrundlagen (Bereinigte vs. Bruttoausgaben) resultieren und kann zum anderen auch dahingehend interpretiert werden, daß kleinere Kommunen im Betrachtungsjahr mehr investiert haben als die Gemeinden mit mehr als 20.000 Einwohnern.
} 
laufenden an den gesamten Bruttosportausgaben. Die Standardabweichung dieser prozentualen Aufteilung auf laufende und einmalige Mittel beträgt 18,2. Nun können Schwankungen im investiven Anteil bis zu einem gewissen Grad damit erklärt werden, $\mathrm{da} ß$ eine bestimmte Kommune im Betrachtungsjahr mehr (oder auch weniger) als in sonstigen Jahren in den kommunalen oder vereinseigenen Sportstättenbau investiert hat, so z.B. aufgrund einer im Betrachtungsjahr 1991 durchgeführten größeren, länger geplanten Investition, die genauso im Folgejahr hätte erfolgen können.

Um jegliche Zufälligkeiten des Referenzjahres 1991 auszuschalten, werden im weiteren Verlauf lediglich die laufenden Ausgaben berücksichtigt. Ferner beschränkt sich der Untersucher auf die Ausgaben für die Förderung des Sports und für Sportstätten, da etliche Kommunen keine Angaben zu den Badeanstalten machten. ${ }^{153}$

\section{Abbildung 16: Die laufenden Bruttoausgaben für Sportförderung und Sportstät- ten 1991 in MIO DM im Querschnitt}

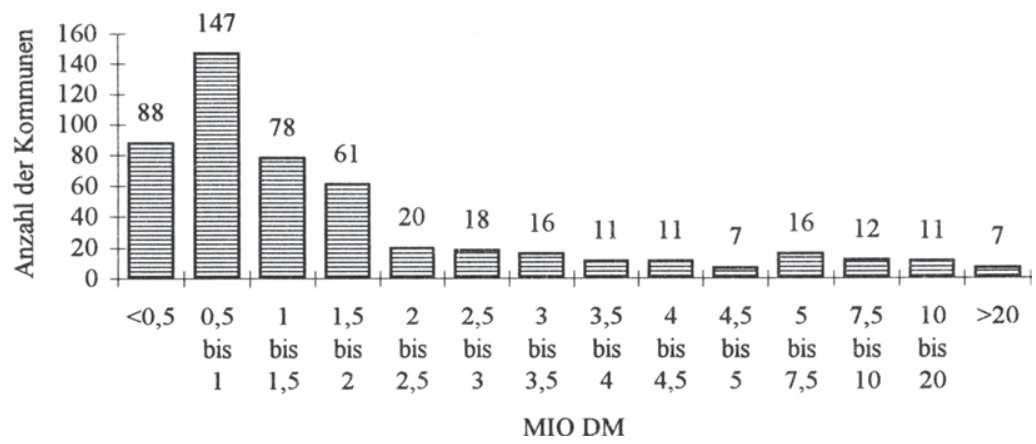

Die Spanne reicht, wie anhand von Abbildung 16 für das Betrachtungsjahr 1991 ersichtlich wird, von unter DM 0,5 Millionen bis hin zu über DM 50 Millionen.

Dem kann entgegengehalten werden, daß es nicht verwunderlich ist, wenn die Sportausgaben einer Millionenmetropole jene einer Kleinstadt mit gerade einmal gut 20.000 Einwohnern übersteigen. Diese in Richtung eines potentiellen linearen Zusammen-

\footnotetext{
${ }^{153}$ Es handelt sich hierbei um jene Kommunen, die die Ausgaben für Badeanstalten nicht innerhalb des Einzelplanes 5 ausweisen.
} 
hangs zwischen Gemeindegröße und Sportausgaben zielende Aussage, soll, ohne an dieser Stelle bereits zu sehr auf die Frage nach den Determinanten des Sportausgabeniveaus einzugehen, anhand von Abbildung 17 berücksichtigt werden, welche die Verteilung der Pro-Kopf-Ausgaben ausweist.

Auch die Betrachtung der laufenden Pro-Kopf-Ausgaben zeigt eine ähnlich starke Spanne wie die zuvor betrachteten Gesamtausgaben, womit deutlich wird, daß die Einwohnerzahlen allein die beträchtlichen Unterschiede im Gesamtausgabeniveau zwischen den einzelnen Kommunen nicht hinreichend erklären können. So differieren selbst die Pro-Kopf-Ausgaben im Verwaltungshaushalt von unter DM 3,- bis hin zu etwa DM 200,-. Das arithmetische Mittel der laufenden Pro-Kopf-Ausgaben des Betrachtungsjahres 1991 für Förderung des Sports und Sportstätten beträgt DM 34,6, die Standardabweichung 24,9 und der Variationskoeffizient beläuft sich somit auf 0,72. Damit kann kaum von einer Homogenität der Sportausgaben gesprochen werden.

\section{Abbildung 17: Die laufenden Bruttoausgaben für Sportförderung und Sportstät- ten 1991 in DM pro Kopf im Querschnitt}

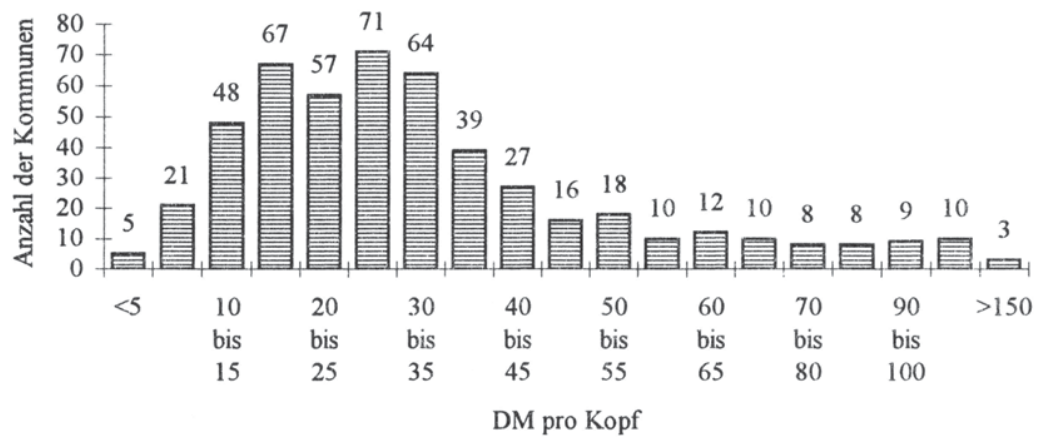

Ein weiterer möglicher Einwand könnte sich darauf beziehen, daß einige Kommunen für bestimmte Sportstätten oder auch generell für alle Sportstätten fiktive Wertansätze für die Sportstättenüberlassung, kalkulatorische Abschreibungen oder auch kalkulatorische Zinsen in Ansatz bringen, damit den Sportetat c.p. erhöhen und die abgeleiteten Aussagen damit abschwächen. ${ }^{154}$ Wenngleich es sich dabei - zumindest was die

\footnotetext{
${ }^{154} \mathrm{Vgl}$. die Ausführungen in Abschnitt 2.1.4.
} 
Haushaltsabschnitte 550 und 560 anbelangt - eher um Ausnahmen handelt und ein Ansatz kalkulatorischer Kosten keineswegs eine derart hohe Streuung erklären kann, wurden in einem weiteren Schritt die laufenden direkten Zuwendungen an Sportvereine betrachtet, die in Abbildung 18 dargestellt werden. ${ }^{155}$ Hierbei handelt es sich um eine Ausgabeposition, die keinerlei kalkulatorische Aufwendungen kennt, womit eine vollständige Vergleichbarkeit der Kommunen gegeben ist.

\section{Abbildung 18: Die laufenden direkten Zuwendungen an Sportvereine 1991 in DM pro Kopf im Querschnitt}

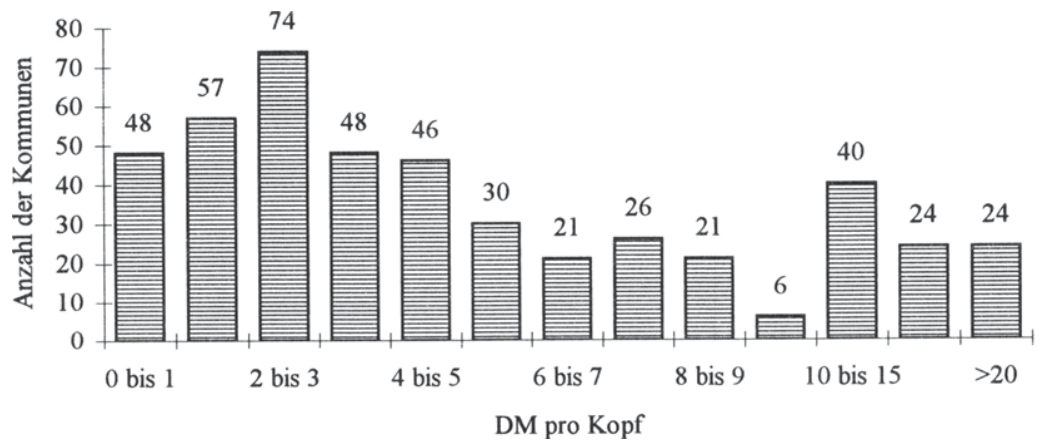

Es zeigte sich ein ähnliches Bild, wie es auch schon die Untersuchung der gesamten laufenden Kosten abgeliefert hatte. Die Kommunen zeichnen sich durch beträchtliche Unterschiede in der Vergabe direkter Pro-Kopf-Gelder an die Sportvereine aus. Dies wird umso deutlicher als die Standardabweichung der laufenden Pro-Kopf-Zuschüsse $(6,58)$ das arithmetische Mittel $(6,3)$ sogar übersteigt und der Variationskoeffizient somit einen Wert über 1 annimmt $(1,04)$. Die bereits in Abschnitt 2.1.5. im Rahmen der Betrachtung kommunaler Sportstättenbestände beobachteten, starken interkommunalen Unterschiede konnten durch die Analyse der verschiedenen Ausgabevolumina bestätigt werden.

\footnotetext{
${ }^{155}$ Im Zusammenhang mit den Badeanstalten wird, wie weiter oben dargestellt, wesentlich häufiger auf einen Ansatz kalkulatorischer Kosten zurückgegriffen. Die Badeanstalten sind aber ohnehin nicht $\mathrm{Be}-$ standteil dieser Querschnittsbetrachtung.
} 
Es drängt sich folglich auch die Frage nach den Gründen dieser Uneinheitlichkeit kommunaler Sportpolitik auf, die an späterer Stelle zu beantworten versucht wird.

\subsection{Die Sportförderung der Länder}

\subsubsection{Träger der Sportpolitik}

Die Ansiedlung der mit der Sportpolitik beauftragten Ressorts differiert zwischen den einzelnen Ländern. Zumeist obliegt sie dem Kultusministerium, in einigen Bundesländern existieren eigens eingerichtete Sportministerien.

Abbildung 19: Zuständigkeiten für den Sport in den Landesregierungen der Flächenbundesländer

\begin{tabular}{|l|l|}
\hline Bundesland & für den Sport zuständiges Ressort innerhalb der Landesregierung \\
\hline Baden-Württemberg & Ministerium für Kultus und Sport \\
\hline Bayern & Staatsministerium für Unterricht, Kultus, Wissenschaft und Kunst \\
\hline Hessen & Ministerium des Innern und für Europaangelegenheiten \\
\hline Niedersachsen & Kultusministerium \\
\hline Nordrhein-Westfalen & Kultusministerium des Landes NRW \\
\hline Rheinland-Pfalz & Ministerium des Innern und für Sport \\
\hline Saarland & Ministerium für Bildung und Sport \\
\hline Schleswig-Holstein & $\begin{array}{l}\text { Ministerium für Frauen, Bildung, Weiterbildung und Sport des } \\
\text { Landes Schleswig-Holstein }\end{array}$ \\
\hline
\end{tabular}

Neben diesen in Abbildung 19 aufgeführten federführenden bzw. koordinierenden Ministerien der Flächenbundesländer sind zumeist noch einige andere Ressorts mit Randaufgaben des Sports betraut. ${ }^{156}$

Die Entscheidungen, die für die Bereiche des Sports letztlich in den betreffenden Ministerien getroffen werden, werden im allgemeinen in den sogenannten Landessportkonferenzen vorbereitet. Diese Gremien, 157 in denen staatliche und kommunale Stellen sowie die Parteien und Sportvertreter an einem Tisch sitzen, geben Empfehlungen an die verschiedenen Behörden.

${ }^{156} \mathrm{Vgl}$. Land Baden-Württemberg, Landessportpläne. Ähnlich in Hessen, wo neben dem federführenden Ministerium des Innem und für Europaangelegenheiten auch das Ministerium für Jugend, Familie und Gesundheit, das Kultusministerium, das Ministerium für Wissenschaft und Kunst, das Justizministerium und schließlich das Ministerium für Landesentwicklung, Wohnen, Landwirtschaft, Forsten und Naturschutz Teilaufgaben auf dem Sportsektor wahrnehmen. Vgl. Land Hessen, 1992.

${ }^{157} \mathrm{Die}$ Landessportkonferenzen können als Äquivalent zur deutschen Sportkonferenz angesehen werden. 
Um die Strukturen der Sportförderung der einzelnen Länder nicht allzuweit auseinanderklaffen zu lassen, wurde 1977 auf Bundesebene die Konferenz der Sportminister der Länder (SMK) gebildet, die als Instrument der Koordinierung der Sportförderungsmaßnahmen in den Ländern gilt. ${ }^{158} \mathrm{Ihr}$ gehören 15 Länderminister oder -senatoren sowie der Bundesinnenminister als Gast an. ${ }^{159}$

Die kommunalen Spitzenverbände und der Deutsche Sportbund als Wortführer der Interessen des organisierten Sports werden regelmäßig zur Konferenz eingeladen, ${ }^{160}$ Vertreter alternativer Sportangebotsformen sind hierbei nicht repräsentiert.

\subsubsection{Legislative Grundlagen - Gesetze, Leitlinien und Verordnungen}

Gemäß Art. 30 Grundgesetz ist die Ausübung der staatlichen Befugnisse und die Erfüllung der staatlichen Aufgaben Sache der Länder, soweit dieses Grundgesetz keine andere Regelung trifft oder zuläßt.

Innerhalb der verschiedenen Bundesländer ist die Förderung des Sports nun unterschiedlich geregelt, es existierten bis 1989 elf unterschiedliche Förderprogramme bzw. -strukturen, die einen Vergleich zwischen den Ländern erschweren.

Eine Regelung, die im Rahmen der öffentlichen Sportförderung bislang nur auf Länderebene in Erscheinung getreten ist, stellen die Sportfördergesetze dar, die seit 1974 in Rheinland-Pfalz und seit 1976 bzw. 78 in Bremen und Berlin existieren. ${ }^{161}$ Diese Gesetze zeichnen sich in erster Linie dadurch aus, daß sie - ähnlich den Sportförderrichtlinien auf kommunaler Ebene - die Förderungsmaßnahmen und -adressaten abgrenzen als auch die Funktions-, Verfahrens- und Kooperationsprinzipien öffentlicher Institutionen im Bereich der Sportförderung festlegen. Einen eindeutig quantifizierbaren Anspruch des Sports gibt allerdings keines der drei Gesetze, vielmehr wird auf eine Abhängigkeit der Sportförderung von der jeweiligen Haushaltssituation hingewiesen. ${ }^{162}$

${ }^{158}$ Diesem Gremium soll hier eine Doppelfunktion als indirekter Träger der Sportpolitik sowie als Institution zur Erarbeitung von Leitlinien zugeteilt werden.

${ }^{159}$ Diese Angaben beziehen sich auf die alten Bundesländer und setzen sich zusammen aus 11 Kultusbzw. Sportministern und vier Innen- bzw. Sozjal- oder Jugendministern/-senatoren.

${ }^{160} \mathrm{Vgl}$. Bundesministerium des Innern, 1991, S.20.

${ }^{161}$ Landesgesetz über die öffentliche Förderung von Sport und Spiel in Rheinland-Pfalz (vom 9.12.1974); Gesetz zur Förderung des Sports im Lande Bremen (vom 5.7.1976); Gesetz zur Förderung des Sports im Lande Berlin (vom 12.10.1978).

Eine ausführliche Kommentierung und Analyse dieser Gesetze findet sich bei Güldenpfennig, 1980, Teil 3, S. $106 \mathrm{ff}$.

${ }^{162} \mathrm{Vgl}$. Güldenpfennig, 1980, S. 106 ff. Der Verweis auf eine Abhängigkeit der Sportgelder von der jeweiligen Haushaltslage wurde bereits innerhalb der kommunalen Sportförderrichtlinien, letztlich dem Pendant zu den o.g. Sportfördergesetzen, explizit dargestellt. Es soll an späterer Stelle sowohl die tatsächliche Relevanz dieser budgetären Restriktion überprüft als auch hinterfragt werden, inwieweit 


\subsubsection{Länder-Haushalte und Sportförderung}

Länder-Haushalte sind neben gesonderten Positionen für den Landtag, den Ministerpräsidenten, den Landesrechnungshof etc. in Einzelpläne für die jeweiligen Ministerien untergliedert. Mittel für den Sport werden folglich primär im Einzelplan des jeweils federführenden Ressorts ausgewiesen. ${ }^{163}$ Weitere, dem Sport zuzuordnende Gelder tauchen in den Einzelplänen jener Ministerien auf, die darüber hinaus mit Randaufgaben auf dem Sektor des Sports betraut sind. ${ }^{164}$

\subsubsection{Qualitative Aspekte der Sportförderung der Bundesländer}

\subsubsection{Unterstützung des kommunalen und vereinseigenen Sportstätten- baus}

Sportpolitik auf Landesebene erstreckt sich zu einem großen Teil auf die Gewährung von Zuschüssen für den allgemeinen Sportstättenbau. In diesem Zusammenhang sind zuallererst die anteiligen Finanzierungen kommunalen Sportstättenbaus durch Zweckzuweisungen zu nennen. Je nach Bedeutung der jeweiligen Sportstätte können solche Zuschüsse bis zu $50 \%$ der Gesamtkosten betragen. So teilten sich, wie weiter oben dargestellt, das Land Baden-Württemberg und die Stadt Stuttgart die Kosten Ausbau des Gottlieb-Daimler-Stadions zu etwa gleichen Anteilen.165 Zum Bau eher freizeitund breitensportorientierter kommunaler Sportstätten werden in Baden-Württemberg in aller Regel Zuschüsse bis zu 30\% der zuschußfähigen Gesamtkosten gewährt. ${ }^{166} \mathrm{An}$ den Folgekosten der Investitionen beteiligen sich die Länder grundsätzlich nicht.

Auf die Bedeutung dieser, nur für investive Maßnahmen bestimmten Zweckzuweisungen wird weiter unten im Rahmen der theoretischen Ausführungen näher eingegangen. Daneben fördern die Bundesländer den Vereinssportstättenbau durch direkte Zuwendungen an Sportvereine und unter Umständen auch Sportfachverbände. Derartige Landeszuschüsse belaufen sich auf bis zu $30 \%$ der jeweiligen Finanzierungslasten.

zusätzliche Bestimmungsfaktoren theoretisch denkbar sind bzw. welche Faktoren die Ausgaben für den Sport de facto bestimmen.

${ }^{163}$ In Baden-Württemberg ist dies bspw. der Einzelplan 04, Ministerium für Kultus und Sport.

${ }^{164}$ Auf eine Darstellung im einzelnen soll aufgrund der unterschiedlichen Haushaltssystematiken verzichtet werden. Zum Sport in den Länderhaushalten, vgl. u.a. Land Baden-Württemberg, Landessportplan 1991/92, S. 17, Land Nordrhein-Westfalen, Landessportplan 1991, S. $461 \mathrm{ff}$.

165 Ähnlich in Karlsruhe und auch Mannheim, wo das Land beschlossen hat, für den Ausbau der dortigen Großstadien einen Zuschuß in Höhe von $50 \%$ der zwischenzeitlich geschätzten Gesamtkosten zu gewähren. Vgl. Land Baden-Württemberg, 1991/92, S. 12. Laut Auskunft des Karlsruher Sportamtes betrug dieser im Endeffekt knapp ein Drittel, die Gesamtkosten überstiegen die zwischenzeitlichen Schätzungen von 25 Millionen um etwa $100 \%$.

${ }^{166} \mathrm{Vgl}$. Land Baden-Württemberg, Landessportplan 1991/92, S.19. 


\subsubsection{Laufende Förderung des Vereinssports}

Mittel für laufende Kosten der Vereinstätigkeit werden von Länderebene in aller Regel gewährt für den Kauf von Sportgeräten, für Fahrten zu Wettkämpfen, kleinere Sportstättenmaßnahmen, Unterhaltung von vereinseigenen Anlagen und, zu einem nicht unbedeutenden Anteil, für die Aus- und Fortbildung sowie Honorierung von Übungsleitern. 167

\subsubsection{Sonstige Förderungsaspekte}

Last not least werden Gelder für speziell spitzensportorientierte Einrichtungen wie Bau und Unterhaltung von Bundes- und Landesleistungszentren, 168 Talentsichtung etc. verausgabt. 169

Zusammenfassend kann festgehalten werden, daß sich die Sportförderung von Ländern und Gemeinden rein qualitativ stark ähneln. Wie auf gemeindlicher zeigt sich darüber hinaus auch auf Landesebene, daß direkte öffentliche Zuwendungen nur dem organisierten Sport zur Verfügung gestellt werden.

${ }^{167} \mathrm{Vgl}$. Schmidt, 1983, S. 157.

${ }^{168}$ Die Finanzierung erfolgt hierbei anteilig zwischen Bund und Ländern, jeweils gemäß der Nutzung der entsprechenden Gebietskörperschaft. Eine Darstellung der Finanzierungsanteile findet sich bei Dellmann, 1983, S. $144 \mathrm{f}$.

${ }^{169} \mathrm{Vgl}$. Schmidt, 1983, S. $151 \mathrm{ff}$. 


\subsubsection{Quantitative Aspekte der Sportförderung der Bundesländer}

\subsubsection{Die Entwicklung der Sportausgaben der Bundesländer von 1965 bis 1991170}

Abbildung 20 zeigt zunächst die Entwicklung der Nettosportausgaben der Bundesländer für Sportförderung, Sportstätten und Badeanstalten. Wie auf Gemeindeebene ist ein tendenzieller Anstieg der Sportausgaben im Betrachtungszeitraum bis hin zu DM 957 MIO im Jahr 1991 zu erkennen. ${ }^{171}$

\section{Abbildung 20: Nettosportausgaben der Bundesländer (incl. Stadtstaaten) von 1965 bis 1991 in MIO DM ${ }^{172}$}

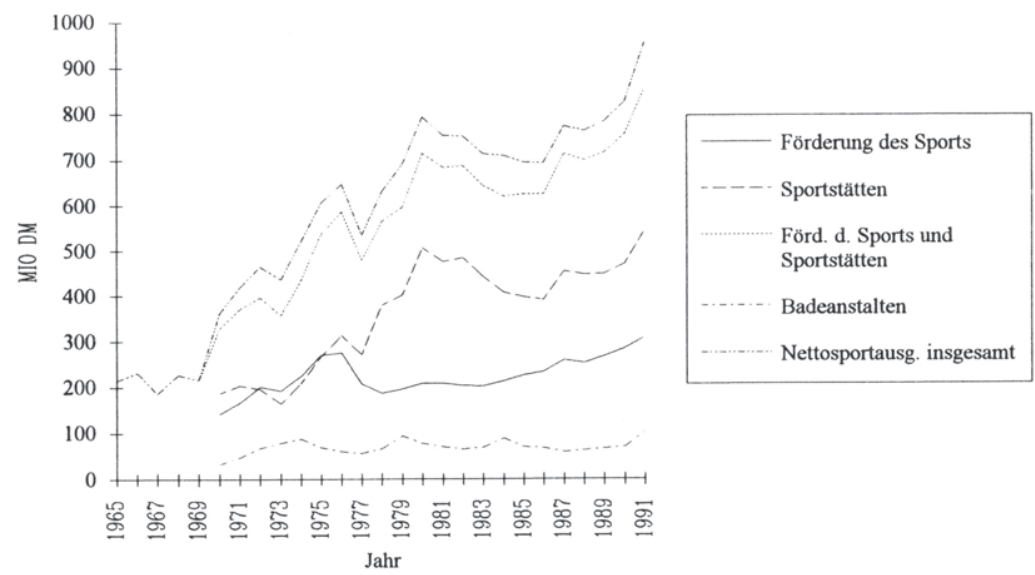

Quelle: Statistisches Bundesamt, Finanzen und Steuern, verschiedene Reihen, Jahrgänge 1965 bis 1991.

\footnotetext{
${ }^{170}$ In Analogie zur Darstellung der Sportforderung der Kommunen betrachtet auch die Länderentwicklung den Zeitraum von 1965 bis 1991 und bezieht sich ebenfalls auf die Angaben des Statistischen Bundesamts. Vgl. Statistisches Bundesamt, Finanzen und Steuern, verschiedene Reihen und Jahrgänge. ${ }^{171}$ Diese DM 957 Millionen im Rechnungsjahr 1991 schließen allerdings die Stadtstaaten mit ein, deren Anteil an dem genannten Betrag immerhin DM 423 Millionen beträgt. Davon gibt allein das Land Berlin DM 333 Millionen aus.

172Ein Ausweis der Landesmittel für Badeanstalten erfolgt erst seit dem Rechnungsjahr 1970. In ähnlicher Weise waren vor 1970 die Ausgaben für Förderung des Sports und Sportstätten zusammengefaßt.
} 
Dieser Trend ist allerdings, mehr noch als auf kommunaler Ebene, etlichen Schwankungen unterworfen. ${ }^{173}$ Auffallend sind insbesondere die kurzfristig nominalen Ausgabereduzierungen in den Jahren 1967, 69, 73 und 77 sowie eine längerfristige Senkung der Sportausgaben in der ersten Hälfte der 80er Jahre.

Vergleicht man den Anteil der verschiedenen Sportmittel an den gesamten Nettoausgaben, dann zeigt sich ein im Vergleich zur kommunalen Ebene wesentlich geringerer Anteil der Ausgaben für Badeanstalten. Abbildung 20 signalisiert überdies einen relativ kontinuierlichen Verlauf der Ausgaben für Förderung des Sports, die lediglich eine größere Trendabweichung Mitte der 70er Jahre aufweisen.

Die oben genannten, zwischenzeitlichen Ausgabereduzierungen beruhen damit offensichtlich primär auf Schwankungen in den Ausgaben für Sportstätten, welche auf Landesebene fast ausnahmslos investiver Natur sind. Ähnlich wie für die kommunale kann damit auch für die Ebene der Länder festgehalten werden, daß Schwankungen im Zeitablauf in erster Linie auf Unregelmäßigkeiten in den investiven Ausgaben fußen.

$\mathrm{Da}$ nun der Anteil der Investitionen an den für die Schwankungen verantwortlichen Sportstättenausgaben und auch an den gesamten Sportausgaben der Bundesländer recht groß ist, können damit auch die im Vergleich zu den Kommunen merklicheren Schwankungen im Zeitablauf erklärt werden. ${ }^{174}$

Abbildung 21 zeigt abschließend die preisbereinigte Entwicklung der Ausgaben. Wie auf kommunaler ist auch auf Landesebene der Höchststand realer Sportförderung mit dem Jahr 1980 überschritten gewesen, was auf die reduzierten Ausgaben für Sportstätten und damit in erster Linie auf reduzierte Investitionszuweisungen für Kommunen und Zuschüsse an Sportvereine zurückzuführen ist. Der prozentuale Anteil ersterer an den gesamten Nettosportausgaben der Bundesländer belief sich phasenweise auf über $50 \%$, seit Anfang der 80er Jahre sinkt dieser Anteil kontinuierlich. ${ }^{175}$

\footnotetext{
${ }^{173}$ Eine Gegenüberstellung der Entwicklung der Ausgaben sämtlicher Gebietskörperschaften erfolgt im Rahmen von Abschnitt 2.4 .

${ }^{174}$ Weiter oben wurde festgehalten, daß die Kommunen 1991 nur gut $20 \%$ ihrer Sportmittel für investive Zwecke verausgabten. Auf Länderebene hingegen betrug der Anteil der investiven Maßnahmen im selben Jahr immerhin etwa 50\%. Berücksichtigt man nur die für die Schwankungen verantwortlichen Sportstättenausgaben, so betrug die "Investitionsquote" sogar $80 \%$.
}

${ }^{175} \mathrm{Vgl}$. hierzu Anhang 5. 
Abbildung 21: Nettosportausgaben der Bundesländer (incl. Stadtstaaten) von 1965 bis 1991 in MIO DM, nominal und real, Basisjahr 1985

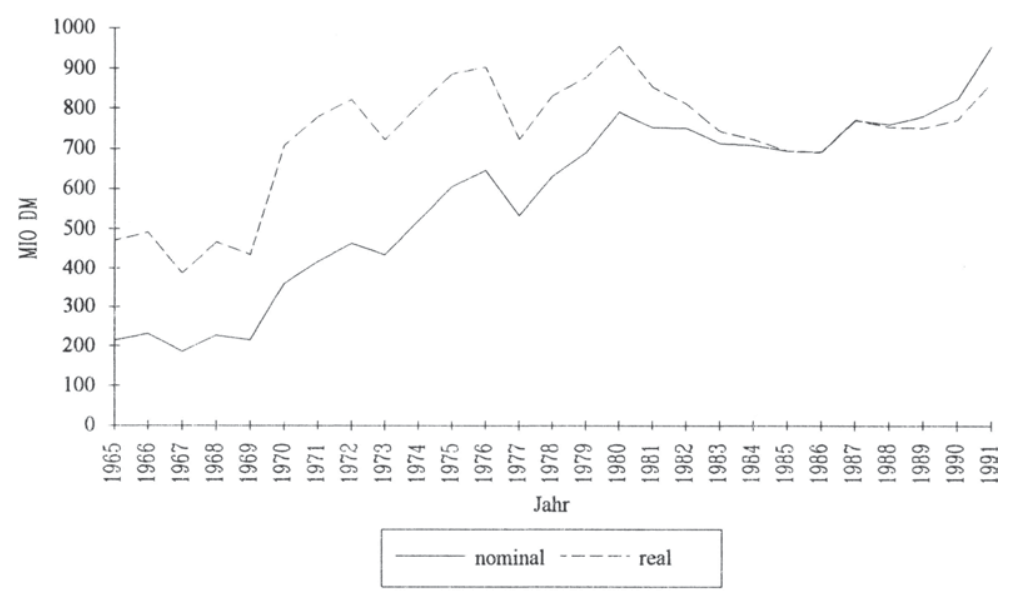

Quelle: Statistisches Bundesamt, Finanzen und Steuern, verschiedene Reihen, Jahrgänge 1965 bis 1991. 


\subsubsection{Die Sportausgaben der Bundesländer im Jahr $1991 \mathrm{im}$ Querschnitt}

Die Untersuchung der Sportausgaben der Kommunen im Querschnitt offenbarte große Diskrepanzen. Ähnliches läßt sich bei Betrachtung von Abbildung 22 auch für die Bundesländer festhalten. Schließt man die Stadtstaaten Berlin, Bremen und Hamburg aufgrund ihrer Doppelfunktion als Länder und Kommune aus, dann zeigt sich, daß das Land Baden-Württemberg, gefolgt von Rheinland-Pfalz und Bayern, pro Einwohner die meisten Gelder für den Sport verausgabt.

\section{Abbildung 22: Die Nettosportausgaben der Flächenbundesländer 1991 in DM pro Kopf im Vergleich}

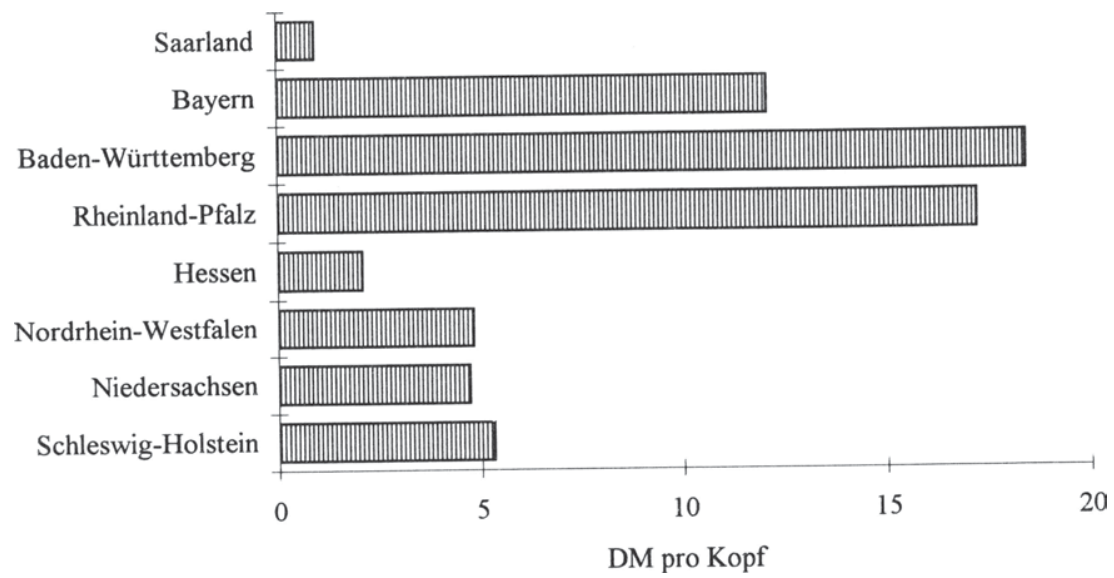

Quelle: Statistisches Bundesamt, Finanzen und Steuern, verschiedene Reihen, Jahrgänge 1965 bis 1991.

Interessanterweise handelt es sich hierbei keineswegs um ein Phänomen eines bestimmten Jahres, sondern vielmehr um eine über einen längeren Zeitraum hinweg zu beobachtende beträchtliche Unterscheidung bezüglich des Pro-Kopf-Ausgabevolumens. 


\subsection{Die Sportförderung des Bundes}

\subsubsection{Träger der Sportpolitik}

Auf Bundesebene sind im wesentlichen Bundesregierung und Bundestag für Fragen des Sports zuständig. Innerhalb der Bundesregierung ist der Sport vorrangig Angelegenheit des Bundesministeriums des Innern, welches alle sportrelevanten Maßnahmen auf Regierungsebene koordiniert. Daneben besitzen zehn weitere Ressorts im Rahmen ihrer Aufgabenstellung Teilzuständigkeiten für die Sportförderung. ${ }^{176}$ Träger der Sportpolitik auf der legislativen Ebene ist der Sportausschuß des Bundestags.

\subsubsection{Legislative Grundlagen - Gesetze, Leitlinien und Verordnungen}

Spezielle gesetzliche Regelungen für die Sportpolitik des Bundes existieren nicht. Das Grundgesetz enthält zwar einige Bestimmungen, die für Teilgebiete des Sports relevant sind, ${ }^{177}$ die Vergabe der Mittel erfolgt jedoch vorwiegend nach dem 1974 erstmals erstellten und mittlerweile überarbeiteten Leistungssportprogramm der Bundesregierung ${ }^{178}$ sowie einigen weiteren Vorschriften und allgemeinen Richtlinien, die dieses Leistungsprogramm konkretisieren. 179

Eine von den Sportreferenten von Bund und Ländern getroffene Vereinbarung regelt darüber hinaus den Finanzierungsanteil beider Gebietskörperschaften im Rahmen der Gemeinschaftsausgaben ${ }^{180}$ und ferner enthält ein von einer Bund/Länder-Verhandlungskommission erarbeiteter Entwurf über die Finanzierung öffentlicher Aufgaben

\footnotetext{
${ }^{176}$ So ist bspw. das Verteidigungsministerium für den Sport innerhalb der Bundeswehr zuständig. Eine vollständige Auflistung aller, im Rahmen der Bundesregierung mit sportbezogenen Maßnahmen betrauten Ressorts findet sich im Sportbericht der Bundesregierung, vgl. Bundesministerium des Innern, 1991, S. 39.

${ }^{177}$ Es sind dies im einzelnen:

-Art. 2, Abs. I GG für die freie Entfaltung der Persönlichkeit, -Art. 9, Abs. 1 GG für die Vereinigungsfreiheit,

-Art. 32 GG für die Pflege der Beziehungen zu auswärtigen Staaten,

-Art. 9 la GG für den Hochschulbau und die Verbesserung der regionalen Wirtschaftsstruktur,

-Art. $91 \mathrm{lb}$ GG für die Bildungsplanung und die überregionale Forschungsförderung.

-Art. 104a, Abs. 4 GG für Finanzhilfen im Zusammenhang mit dem Städtebauförderungsgesetz.

${ }^{178} \mathrm{Vgl}$. Bundesministerium des Innern, 1991, S. $176 \mathrm{ff}$.

${ }^{179}$ Hierzu gehören in erster Linie die Grundsätze für die Planung, Errichtung, Benutzung, Unterhaltung und Verwaltung von Bundesleistungszentren, die Grundsätze für die Förderung der Errichtung von Landesleistungszentren durch den Bund sowie über das Förderungsverfahren, die Grundsätze für die Anerkennung von Stützpunkten als auch die Grundsätze für die Aufstellung von Strukturplänen, vgl. Bundesministerium des Innern, S. $185 \mathrm{ff}$.

${ }^{180}$ Der Förderungsanteil des Bundes variiert je nach Grad der Bundesnutzung; eine detaillierte Übersicht über den Finanzierungsanteil an verschiedenen Aufgaben findet sich bei Dellmann 1983, S. $144 \mathrm{ff}$.
} 
von Bund und Ländern, das sogenannte Flurbereinigungsabkommen, einige sportrelevante Bestimmungen. ${ }^{181}$

Im Zusammenhang mit den legislativen Grundlagen der Sportpolitik ist schließlich noch auf die Sportprogramme der politischen Parteien hinzuweisen, die allerdings keineswegs speziell auf die Sportförderung des Bundes gerichtet sind.

Eine Betrachtung derselben zeigte keine nennenswerten Unterschiede hinsichtlich der Grundsatzempfehlungen zur Sportförderung der Gebietskörperschaften, so daß hieraus im Prinzip keine Unterschiede in der Sportpolitik von Entscheidungsgremien verschiedener politischer Couleur zu erwarten sind. ${ }^{182}$

\subsubsection{Sport im Bundeshaushalt}

Ähnlich den Etats der Länder ist auch der Bundeshaushalt in Einzelpläne für Bundestag, -präsident, -kanzler und die verschiedenen Ministerien untergliedert. Sportmittel werden primär im Einzelplan des federführenden Bundesministeriums des Innern erfaßt. Weitere, dem Sport zurechenbare Gelder werden in den Einzelplänen jener Ressorts verbucht, die darüber hinaus mit sportbezogenen Aufgaben betraut sind.

\subsubsection{Qualitative Aspekte der Sportförderung des Bundes}

Hauptobjekte der Sportpolitik des Bundes sind Unterstützungen des Hochleistungssports bzw. Förderungen jener Aktivitäten, die nationalen oder grenzüberschreitenden Charakter haben. ${ }^{183}$

Konkret richtet sich diese Politik auf Sportstätten, die dem Hochleistungssport optimale Trainingsbedingungen garantieren. So finanziert der Bund mit einem je nach Umfang der Bundesnutzung variierenden Anteil den Bau und die Unterhaltung von Bundesleistungszentren und Landesleistungszentren mit Bundesnutzung.

\footnotetext{
${ }^{181} \mathrm{Im}$ Bereich der Sportförderung ist der Bund nach dem Flurbereinigungsabkommen vorrangig zuständig für Auslandsbeziehungen, gesamtstaatliche Repräsentation, Förderung von Maßnahmen nichtstaatlicher zentraler Organisationen, die fuir das Bundesgebiet als Ganzes von Bedeutung sind und ferner für diverse ressortzugehörige Funktionen.

182Vgl. hierzu Die Grünen 1986, CDU 1993, FDP 1993 und SPD 1993. Eine ausführliche Darstellung der verschiedenen Sportprogramme, allerdings etwas älterer Auflagen, findet sich bei Schmidt, 1979.

${ }^{183}$ Eine sehr ausfuhrliche Schilderung einzelner Fördermaßnahmen findet sich in den Sportberichten der Bundesregierung, die seit 1970 vom Bundesminister des Innern herausgegeben werden. Derzeit aktuell ist der siebte Sportbericht der Bundesregierung aus dem Jahr 1991. Vgl. Bundesministerium des Innern, 1991.
} 
Im Rahmen der Talentsuche fördert der Bund zentrale Maßnahmen zur Talentsichtung, die Teilnahme von Talenten an bedeutenden Wettbewerben, den Finalwettbewerb der Veranstaltung "Jugend trainiert für Olympia" und dergleichen.

Ein weiterer Punkt ist die indirekte Förderung von Hochleistungssportlern durch Finanzierung von Trainern, Lehrgängen und Stützpunkttraining sowie sozialer und sportmedizinischer Betreuung.

Schließlich sind es die Bereitstellung technischer Hilfsmittel, die Unterstützung der Sportwissenschaft, die grundsätzliche Organisation des Hochleistungssports und nicht zuletzt Zuschüsse zur Finanzierung der Ausrichtung von Wettkämpfen, die den Bereich der Förderung des Hochleistungssports komplettieren. ${ }^{184}$

Breiten- und Freizeitsport werden dort mitgefördert, wo es sich um Bereiche handelt, die, wie bspw. Post oder Bundeswehr, unter die Zuständigkeit des Bundes fallen. ${ }^{185}$

${ }^{184}$ Das bekannteste Beispiel anteiliger Bundesfinanzierung von Spitzensportveranstaltungen waren die im Zusammenhang mit der Ausrichtung der Olympischen Spicle 1972 gewährten Mittel.

${ }^{185}$ Es sind damit unter anderem die verschiedenen, in Bundeswehrkasernen unterhaltenen Sportstätten angesprochen. 


\subsubsection{Quantitative Aspekte - Die Entwicklung der Sportausgaben des Bun- des von 1965 bis 1991}

Im Gegensatz zum Ausgabeniveau bei Ländern und Gemeinden zeichnet sich die Sportpolitik des Bundes durch eine deutlich höhere Stetigkeit aus. Abgesehen von den Jahren 1970 bis 1973 und dem erkennbaren Anstieg im Jahr 1991 verausgabte der Bund im Durchschnitt knapp DM 100 MIO jährlich für den Sport. ${ }^{186}$

\section{Abbildung 23: Die Sportausgaben des Bundes von 1965 bis 1991 in MIO DM}

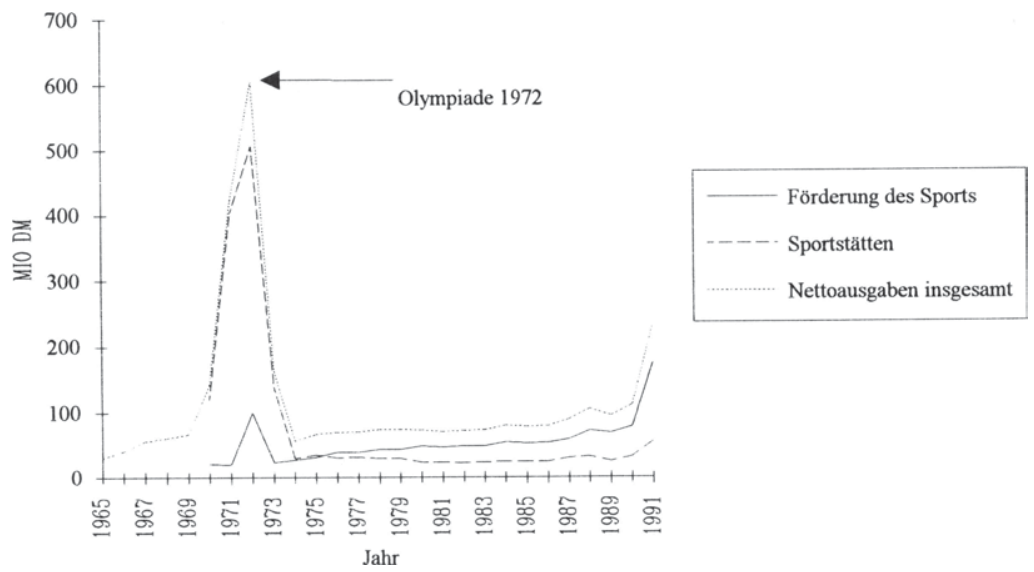

Quelle: Statistisches Bundesamt, Finanzen und Steuern, verschiedene Reihen, Jahrgänge 1965 bis 1991.

Die in Abbildung $23 \mathrm{zu}$ beobachtenden Abweichungen von dieser grundsätzlichen Konstanz in den Jahren 1970 bis 1973, insbesondere die Jahre 1971 und 72, sind auf die im Zusammenhang mit den Olympischen Spielen 1972 in München ${ }^{187}$ und der

\footnotetext{
${ }^{186}$ Diese Werte beruhen auf den Angaben des Statistischen Bundesamtes. Laut Sportberichten der Bundesregierung, die zur Berechnung des Gesamtniveaus neben den Sportgeldern des Bundesministeriums des Innern auch die im Rahmen anderer Einzelpläne verausgabten sportorientierten Mittel hinzurechnen, beläuft sich der jährliche Aufwand auf mehr als das doppelte. Ein Großteil hiervon beruht auf den innerhalb des Verteidigungshaushalts dem Sport zugewiesenen Ausgaben. Vgl. Bundesministerium des Innern, 1991, S. 299.

${ }^{187}$ Neben München waren auch noch Kiel und Augsburg als Austragungsorte für Segel- und Wildwassersport an der Ausrichtung beteiligt.
} 
Vorbereitung der Fußballweltmeisterschaft 1974 entstandenen Kosten zurückzuführen, an denen sich der Bund beteiligt hatte. ${ }^{188}$

\subsection{Der Gesamtumfang öffentlicher Sportausgaben}

Der Gesamtumfang der öffentlichen Sportausgaben gemäß der im Rahmen der Dissertation vorgenommenen Begriffsabgrenzung betrug im Rechnungsjahr 1991 netto etwa DM 6,1 MRD. Eine Hinzunahme der Ausgaben für den Schul- und Hochschulsport sowie weiterer, nicht in den expliziten Sporteinzelplänen verausgabter Mittel, ließe das Gesamtniveau auf schätzungsweise über DM 10 MRD ansteigen. ${ }^{189}$

\section{Abbildung 24: Öffentliche Sportförderung in den Gebietskörperschaften der BRD von 1965 bis 1991 in MIO DM}

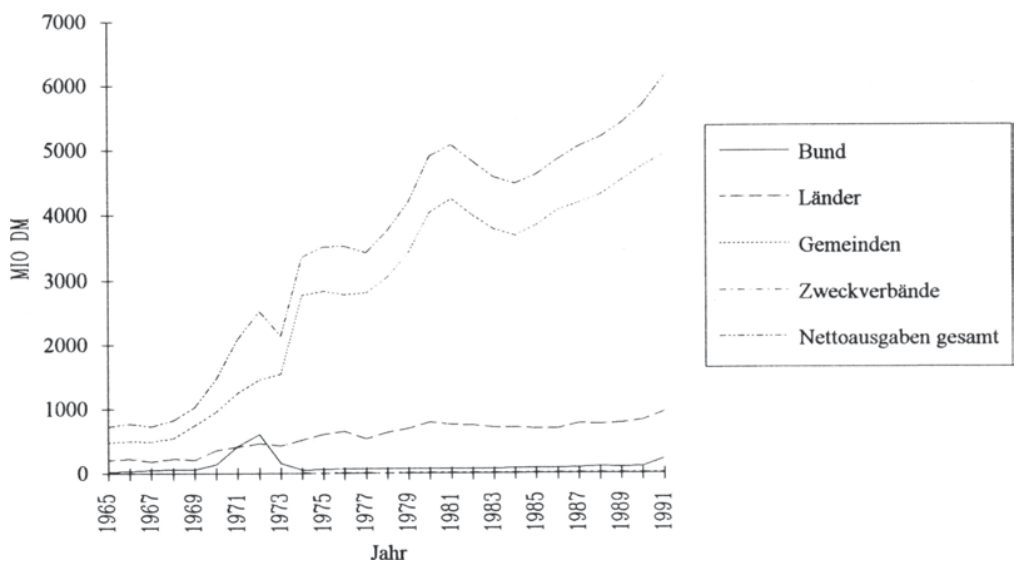

Quelle: Statistisches Bundesamt, Finanzen und Steuern, verschiedene Reihen, Jahrgänge 1965 bis 1991.

\footnotetext{
${ }^{188} \mathrm{Vgl}$. Statistisches Bundesamt, Finanzen und Steuern, 1974, S. 8.

${ }^{18 \%}$ Weber schätzt die gesamten Sportlehrergehälter auf etwa DM 3,3 MRD. Vgl. Weber, 1995, S. 49 Dies entspricht in etwa den Angaben innerhalb des Landessportplanes Nordrhein-Westfalen, welcher die Gehälter der Sportlehrer auf etwa 1/15 der gesamten Lehrergehälter schätzt und dies dann für das Land Nordrhein-Westfalen mit etwa DM 0,75 MRD pro Jahr beziffert. Vgl. Land Nordrhein-Westfalen, 1992, S. 445. Weitere, in den Ausführungen des Statistischen Bundesamts nicht dem Sport zugeordnete Mittel sind bspw. jene Gelder, die in den Länder oder Bundeshaushalten außerhalb der federführenden Ressorts verbucht, in Veröffentlichungen dieser Gebietskörperschaften jedoch zumeist als Sportausgaben ausgewiesen werden.
} 
Der Großteil der in den Sportetats ausgewiesenen Mittel wird von den Kommunen verausgabt; öffentliche Sportförderung ist damit, wie bereits in der Einführung unterstellt, vorrangig eine kommunale Sportförderung. Der prozentuale Anteil der gemeindlichen an den gesamten Sportausgaben der Gebietskörperschaften stieg im Betrachtungszeitraum stark an und beträgt mittlerweile etwa $80 \%$. Splittet man die vom Statistischen Bundesamt als Länderausgaben ausgewiesenen Sportfördermittel der Stadtstaaten in einen gemeindlichen und einen Länderanteil auf und schlägt ersteren dem Ausgabevolumen der Kommunen zu, dann erhöht sich der Kommunalanteil an der gesamten öffentlichen Sportförderung in der Bundesrepublik Deutschland gar auf $87 \%{ }^{190}$

Diese Charakterisierung öffentlicher Sportpolitik als Kommunalpolitik wird umso deutlicher, wenn man das Sportausgabevolumen der verschiedenen Gebietskörperschaften auf die jeweiligen Gesamtausgaben bezieht. ${ }^{191}$ Der Bund verausgabt demzufolge nur etwa 0,05\% seiner Gesamtausgaben für den Sport, bei den Ländern beläuft sich dieser Anteil auf etwa 0,15\%. Die Kommunen verausgaben mit knapp 2\% hingegen einen wesentlich höheren Anteil ihrer Gesamtausgaben für den Sport.

Ein Blick auf die preissteigerungsbereinigten Ausgaben zeigt abermals, daß die bislang meisten Gelder für den Sport im Jahr 1980 verausgabt wurden. Es muß jedoch festgehalten werden, daß der in der zweiten Hälfte der 80 er Jahre zu beobachtende, auf kontinuierlich wachsenden laufenden Kosten beruhende Anstieg zu einem Gesamtniveau geführt hat, welches jenem aus dem Jahre 1980 fast entspricht. Es bleibt abzuwarten, wie sich diese Entwicklung in näherer Zukunft fortsetzen wird. Handelt es sich um einen nur temporären Anstieg der Sportausgaben oder wird der reale Höchststand öffentlicher Sportmittel bald überschritten werden?

\footnotetext{
${ }^{190}$ Die Länderausgaben ohne Berücksichtigung der Stadtstaaten betrugen 1991 netto DM 534 Millionen, jene der Gemeinden DM 4.935 Millionen. Dies entspricht einem Verhältnis von 1:9. Fügt man nun die Ausgaben der Stadtstaaten in Höhe von DM 423 Millionen Ländern und Kommunen gemäß obigem Verhältnis zu, dann ergibt sich für die Kommunen ein Gesamtumfang in Höhe von DM 5.317 Millionen, die Länder verausgabten demzufolge DM 575 Millionen.

${ }^{191}$ Die Angaben zur Höhe der öffentlichen Gesamtausgaben wurden dem Statistischen Jahrbuch des Statistischen Bundesamtes entnommen. Vgl. Statistisches Jahrbuch, 1992.
} 
Abbildung 25: Nominale und reale Sportförderung in der BRD von 1965 bis 1991 in MIO DM, Basisjahr 1985

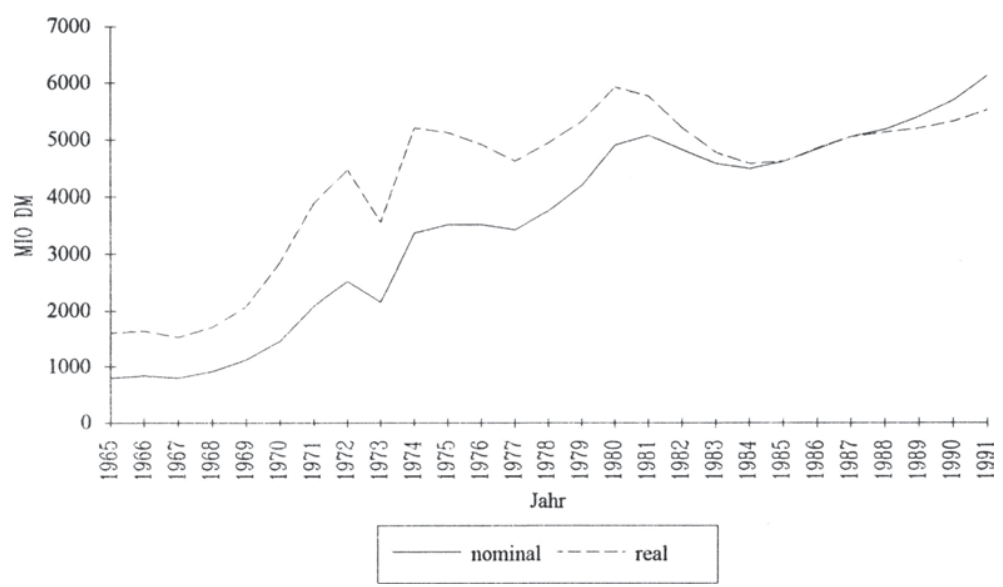

Quelle: Statistisches Bundesamt, Finanzen und Steuern, verschiedene Reihen, Jahrgänge 1965 bis 1991.

Der relative Zuschußbedarf $\mathrm{zu}$ den gesamten, in den Gebietskörperschaften der alten BRD verausgabten Sportmitteln betrug im Jahr 1991 etwa 85\%. Betrachtet man nur die Ausgaben für Förderung des Sports und Sportstätten, dann steigt dieser Anteil an den gesamten Sportausgaben, der letztlich aus öffentlichen Mitteln getragen wird, auf $90 \%$. 


\section{3. Öffentliche Sportförderung durch Steuererleichterungen}

Sportförderung durch Steuererleichterung ist eine Angelegenheit, über die auf Bundesebene im Rahmen der Steuergesetzgebung entschieden wird. Nichtsdestotrotz verzichten mit den diversen Steuererleichterungen, die dem Sport in der BRD zukommen, nicht nur der Bund, sondern auch die Länder und Gemeinden auf potentielle Steuereinnahmen.

So werden Einkommens- bzw. Körperschaftssteuer hauptsächlich zwischen Bund und Ländern aufgeteilt; die Gemeinden erhalten aus diesem Topf nur einen geringen Anteil. Die Umsatzsteuer fließt zu $65 \%$ in Bundes- und zu $35 \%$ in Länderkassen, lediglich die Gewerbesteuer ist in erster Linie eine gemeindliche Steuer, an der Bund und Länder im Rahmen der Gewerbesteuerumlage partizipieren.

Grundvoraussetzung, um in den Genuß verschiedener sportbezogener Steuererleichterungen zu gelangen, ist der Status der Gemeinnützigkeit, welcher den Vereinen des organisierten Sports vorbehalten ist. Daneben differenziert die Steuergesetzgebung nach verschiedenen Vereinsaktivitäten. So erstrecken sich Vergünstigungen bspw. nicht auf Einnahmen im Zusammenhang mit Vereinsgaststätten und Werbung, was laut Bundesministerium der Finanzen eine Wettbewerbsverzerrung gegenüber den voll steuerpflichtigen Unternehmen des Gaststättengewerbes und der Werbewirtschaft verhindern soll. ${ }^{192}$ Von möglichen Wettbewerbsverzerrungen gegenüber den kommerziellen Sportanbietern ist in den Ausführungen des Finanzministeriums nicht die Rede.

Im wesentlichen gestalten sich die diversen Steuererleichterungen derart, daß für Körperschaftssteuer, Gewerbesteuer, Vermögens- und Grundsteuer eine faktische Steuerfreiheit besteht, für die Umsatzsteuer gelten mehrere Steuerermäßigungen.

Gemäß $\S 5,1$ Nr. 9 des Körperschaftssteuergesetzes sind gemeinnützige Sportvereine grundsätzlich von der Körperschaftssteuer befreit. Hiervon profitieren die Vereine in Zusammenhang mit jenen wirtschaftlichen Geschäftsbetrieben, die vom Gesetzgeber als Zweckbetriebe angesehen werden, wozu unter anderem sportliche und gesellige Veranstaltungen zählen, die im Großteil der oftmals ehrenamtlich geführten Vereinen einen nicht unwesentlichen Anteil der Vereinseinnahmen ausmachen. ${ }^{193}$

Auch von der Vermögensteuer sind gemeinnützige Sportvereine nach $§ 3,1 \mathrm{Nr} .12$ des Vermögensteuergesetzes grundsätzlich befreit. Eine Steuerpflicht entsteht erst dann, wenn das Gesamtvermögen des wirtschaftlichen Geschäftsbetriebes die Besteuerungsgrenze von DM 20.000,- überschreitet.

${ }^{192} \mathrm{Vgl}$. Bundesministerium der Finanzen, 1984, S. 11.

${ }^{193}$ Zum Begriff des Zweckbetriebes, vgl. Bundesministerium der Finanzen, 1981, S. 10 f. Als Nichtzweckbetriebe gelten demgegenüber die oben genannten Vereinsgaststätten und Werbeaktivitäten Vgl. ebd. S. 9. 
Die Steuerbefreiung gemeinnütziger Sportvereine von der Gewerbesteuer ist in $\S 3 \mathrm{Nr}$. 6 des Gewerbesteuergesetzes verankert. Eine Ausnahme bilden die wirtschaftlichen Geschäftsbetriebe.

Keine generelle Steuerbefreiung, aber doch einige Steuervergünstigungen erhalten die gemeinnützigen Sportvereine im Rahmen der Umsatzsteuerpflicht. So gilt der ermäBigte Steuersatz von 7\% für die im Rahmen eines Zweckbetriebes ausgeführten Leistungen eines Sportvereins, gänzliche Umsatzsteuerbefreiungen gelten für belehrende Veranstaltungen, Mitgliedsbeiträge u.ä.

Verbleiben einige weitere Vergünstigungen im Rahmen des Einkommensteuergesetzes bezüglich der Aufwandsentschädigung der Übungsleiter sowie durch die Spendenbescheinigungskompetenz der Sportvereine. ${ }^{194}$

Aussagen zum finanziellen Ausmaß dieser dem organisierten Sport gewährten Steuererleichterungen sind kaum durchführbar. ${ }^{195}$ Es kann allenfalls festgehalten werden, daß sich die Gewährung von Steuererleichterungen wie auch schon die Förderung durch öffentliche Ausgaben auf Sportvereine beschränkt, kommerzielle Sportanbieter sind hiervon ausgeschlossen. Insbesondere letztere brachten dem Staat allerdings gemeinsam mit weiteren Unternehmen der Sportbranche ${ }^{196} \mathrm{im} \mathrm{Jahr} 1990$ sportinduzierte Steuereinnahmen in Höhe von etwa DM 5,6 MRD ein. ${ }^{197}$ Davon entfielen DM 2,5 MRD auf nicht abzugsfähige Umsatzsteuereinnahmen, DM 1,7 MRD auf Lohnsteuer, DM 0,8 MRD auf Körperschaftssteuer und DM 0,5 MRD auf Produktionssteuern.

Abschließend läßt sich konstatieren, daß öffentliche Sportförderung zwar vorrangig eine kommunale Aufgabe ist, daß die mit dem Sport einhergehenden Steuereinnahmen allerdings primär Bund und Ländern zukommen.

\footnotetext{
${ }^{194}$ Zur ausführlichen Darstellung der Steuervergünstigungen gemeinnütziger Sportvereine, vgl. Bundesministerium der Finanzen, 1984.

${ }^{195}$ Es wäre zwar denkbar, dic auf die jeweiligen Aktivitäten normalerweise anfallenden Steuern hypothetisch zu beziffern, es ist jedoch kaum vorstellbar, daß der organisierte Sport bei voller steuerlicher Belastung den gleichen Stellenwert hätte. Um zu vernünttigen Schätzungen zu kommen, müßte darüber hinaus auch die Nachfrageelastizität nach Vereinssportgütern bekannt sein.

${ }^{196}$ Hierzu zählen vor allem die Anbieter von Sportwaren wie Sportbekleidung, -schuhe, Sporturlauben und dergleichen.
}

${ }^{197} \mathrm{Vgl}$. Weber, 1995, S. 51. 


\section{Exkurs: Weitere Anmerkungen zum organisierten Sport}

Was sich im Rahmen der Betrachtung öffentlicher Sportfördergrundsätze bereits angedeutet hat, verdichtete sich bei der Darstellung qualitativer Aspekte öffentlicher Sportförderpraxis. Sportförderung in der BRD ist eine Förderung des organisierten Vereinsund Verbandssports.

Sämtliche direkte Zuwendungen richten sich auf diesen Typus der Sportausübung, kommerzielle Sportanbieter partizipieren nicht an diesen Töpfen. Während der vereinsund verbandsorganisierte Sport durch die öffentlichen Hände subventioniert wird, ist die Finanzierung kommerziell betriebener Sportstätten in ihrer Struktur jeder anderen privatwirtschaftlichen Unternehmung vergleichbar. Die einzig denkbare Möglichkeit, in den Genuß öffentlicher Mittel zu kommen, stellen öffentliche Existenzgründungsdarlehen wie das sogenannte Eigenkapitalhilfeprogramm, das ERP Existenzgründungsprogramm oder das Ansparförder- sowie Ergänzungsprogramm der Deutschen Ausgleichsbank dar. Hierbei handelt es sich freilich um Mittel, die nicht speziell dem Sport, sondern jeglichem Unternehmungstypus zur Verfügung gestellt werden können.

Diese Sonderstellung des organisierten Sports als alleinigem Empfänger öffentlicher Gelder wird im Rahmen der theoretischen Ausführungen zur öffentlichen Sportförderung diskutiert, stellt sich doch die berechtigte Frage, wieso bspw. der Tennisspieler in einem Tennisverein mit öffentlichen Mitteln subventioniert wird, derselbe Sportler aber, übt er diesen Sport bei einem kommerziellen Anbieter aus, die vollen Kosten alleine tragen muß.198

Weiter oben wurde festgehalten, daß nur etwa $10 \%$ (ohne Badeanstalten) der dem Sport zur Verfügung gestellten Mittel durch Unmittelbare Einnahmen gedeckt werden. Dem kann möglicherweise entgegengehalten werden, ein Angebot von Sportgütern sei mit den dargestellten Fördermaßnahmen allein nicht möglich, zum Betrieb eines Sportvereins gehöre mehr.

Es erscheint demzufolge angebracht, auf die Haushaltsvolumina der geförderten Sportvereine und -verbände einzugehen und diese mit der Summe der öffentlichen Gelder zu vergleichen. Dabei zeigt sich folgendes Bild:

Weber beziffert das Haushaltsvolumen des organisierten Sports - also der Sportvereine und -verbände - auf etwa DM 7 MRD. ${ }^{199}$ Davon stammen knapp 2/5 von den Mitgliedern (Beiträge, Aufnahmegebühren, Entgelte für spezifische Leistungen des Vereins),

${ }^{198}$ Die weiteren, im Rahmen von Kap. II angesprochenen Sportanbieter erhalten ebenfalls keine direkten Zuwendungen, ihre Förderung beschränkt sich gleichermaßen auf eine - gegenüber dem organisierten Sport allerdings oftmals nachrangige - Mitbenutzung öffentlicher Sportstätten

${ }^{199}$ Vgl. Weber, 1995, S. 46. 
knapp 1/4 der Mittel wird durch eigene wirtschaftliche Tätigkeit sowie durch Einnahmen aus Werbung und Sponsoring und etwa 1/7 durch eigene Veranstaltungen aufgebracht bzw. erwirtschaftet. Fast 1/4 der Einnahmen sind direkte öffentliche Zuschüsse von Bund, Ländern und Kommunen. ${ }^{200}$

Zieht man nun die umfangreichen Infrastrukturleistungen der Bereitstellung von Sportstätten in die Betrachtung mit ein, dann ergibt sich ein wesentlich höherer Anteil. Die Zuschüsse der öffentlichen Hände betragen dann, exklusive der Ausgaben für Badeanstalten, etwa DM 4 MRD. ${ }^{201}$ Dies wiederum entspricht einem prozentualen Anteil an dem um die Infrastrukturleistungen aufgestockten Haushaltsvolumen des organisierten Sports von knapp 50\%. Die gesamten Kosten des Betriebes von Sportvereinen werden mit anderen Worten zu knapp der Hälfte vom Staat und damit in aller Regel aus allgemeinen Steuereinnahmen getragen.

${ }^{200} \mathrm{Vgl}$. die öffentlichen Mittel für die Förderung des Sports, Unterabschnitt 550 im Rahmen der Kommunalhaushalte.

${ }^{201}$ Dieser Betrag ergibt sich durch Abzug der Ausgaben für Badeanstalten von den gesamten Nettosportausgaben. 


\section{Zusammenfassung der Ergebnisse und Darstellung des Erklärungsbe- darfs}

Bevor im Rahmen der theoretischen Erörterungen nach Erklärungsansätzen für die öffentliche Sportförderpraxis gefragt wird, sollen folgende Ergebnisse und der daraus resultierende Erklärungsbedarf zusammengefaßt werden:

\section{a) Die Angebotsstruktur von Sportgütern}

Sportausübung wird in der Bundesrepublik Deutschland zum Großteil von Sportvereinen angeboten, der Anteil der kommerziellen Anbieter zeigt allerdings ansteigende Tendenz.

Es stellt sich folglich unter allokationstheoretischen Gesichtspunkten die Frage nach den Gründen einer derartigen Angebotsstruktur.

\section{b) Gewährung öffentlicher Gelder für den Sport}

Öffentliche Sportförderung beläuft sich gemäß der hier vorgenommenen Begriffsabgrenzung für Förderung des Sports und Sportstätten auf knapp DM 4 MRD netto im Rechnungsjahr 1991. Nimmt man die Ausgaben für Badeanstalten hinzu, dann betragen die Nettoausgaben der öffentlichen Haushalte für den Sport etwa DM 6,1 MRD.

Es stellen sich folgende Fragen: Wieso verausgabt der Staat in einem marktwirtschaftlichen System Gelder für den Sport? Liegen Marktunvollkommenheiten oder gar Marktversagen vor? Lassen sich Zuwendungen pauschal erklären oder bedarf es hierzu einer eventuell differenzierten Betrachtung verschiedener Förderaspekte?

Es konnte darüber hinaus gezeigt werden, daß die öffentlichen Haushalte einen Großteil der im Zusammenhang mit der Sportausübung entstehenden Kosten tragen. Neben der Frage, wieso überhaupt öffentliche Gelder für den Sport gewährt werden, erscheint somit auch deren prozentualer Anteil an den Gesamtkosten bspw. der Bereitstellung von Sportstätten erklärungsbedürftig.

Schließlich konnten zwei grundsätzlich verschiedene Subventionstypen beobachtet werden. So kommen zur Anschubfinanzierung in Form der oben genannten Infrastrukturleistungen oder der Gewährung von Investitionszuschüssen vielfach weitere Gelder für den laufenden Unterhalt vereinseigener Sportstätten sowie sonstige laufende $\mathrm{Zu}$ schüsse für verschiedene Aktivitäten hinzu. Auch diesbezüglich besteht nach Ansicht des Verfassers Erklärungsbedarf. 
c) Öffentliche Sportförderung als Förderung des organisierten Sports

Öffentliche Sportförderung ist abgesehen von der Bereitstellung von Badeanstalten eine fast ausschließliche Förderung des organisierten Vereinssports. Es stellt sich folglich die Frage, wieso die Sportangebote des organisierten Sports subventioniert werden, selbige von alternativen jedoch kaum oder - wie im Falle der kommerziellen Sportanbieter - gar nicht gefördert werden.

d) Öffentliche Sportförderung als primär kommunale Sportförderung Es zeigte sich, daß die Kommunen mit etwa $80 \%$ (incl. Kommunalanteil an den Ausgaben der Stadtstaaten sogar 87\%) für den Großteil öffentlicher Sportfördermittel aufkommen.

Frage: Aus welchen theoretischen Überlegungen heraus läßt sich diese Struktur der Auf- und Ausgabeverteilung auf die Gebietskörperschaften erklären?

\section{e) Starke Unterschiede in Querschnitt und Zeitablauf}

Die Höhe der Sportmittel differiert erheblich innerhalb der verschiedenen Gebietskörperschaften einer jeden föderalen Ebene. Im Zeitablauf zeigten sich beträchtliche Unterschiede zwischen den Investitionsausgaben verschiedener Perioden.

Frage: Wovon hängt die Höhe der dem Sport zugewiesenen öffentlichen Mittel ab, wie lauten die Bestimmungsfaktoren des Niveaus öffentlicher Sportförderung?

Die folgenden Ausführungen nehmen sich dieser Fragen an. Um den Rahmen der Dissertation nicht zu sprengen, muß dies allerdings mit einer unterschiedlich starken Gewichtung erfolgen. 
Christian Hockenjos - 978-3-631-75159-6

Downloaded from PubFactory at 01/11/2019 07:36:54AM

via free access 


\section{Kap. IV Theoretische Erklärungsansätze öffentlicher Sportförderung}

\section{Sport und die Theorie der öffentlichen Güter}

\subsection{Die Theorie der öffentlichen Güter}

\subsubsection{Reine öffentliche Güter und effiziente Allokation}

Die vielzitierte "unsichtbare Hand" ist nicht makellos. Ganz im Gegensatz zu den Annahmen des Modells vollkommener Konkurrenz sind Situationen denkbar, in denen der Markt pareto-optimale Zustände ${ }^{202}$ nicht gewährleisten kann bzw. gleichwohl geschätzte Güter und Dienstleistungen nur in suboptimaler Weise oder gar nicht bereitzustellen in der Lage ist.

Die Theorie der öffentlichen Güter zeigt, warum und inwieweit unter derartigen Umständen eine marktmäßige effiziente Bereitstellung unmöglich ist und beschäftigt sich mit alternativen, dennoch pareto-optimale Zustände ermöglichenden Formen der Bereitstellung solcher Güter. Als reine öffentliche Güter werden dabei jene Güter bezeichnet, die sich durch vollkommene Nichtanwendbarkeit des Ausschlußprinzips sowie durch keinerlei Rivalität im Konsum auszeichnen. ${ }^{203}$

Eine Nichtanwendbarkeit des Ausschlußprinzips kann zum einen erfolgen, wenn die technischen Voraussetzungen, einen potentiellen Konsumenten vom Konsum eines Gutes auszuschließen, nicht gegeben sind. ${ }^{204}$ Zum anderen kann Ausschluß zwar technisch möglich, wirtschaftlich aber wenig sinnvoll sein. Man betrachte einen Joggingpfad, der sich sehr wohl umzäunen ließe und an dessen Eingang ein Kassierer Eintrittsgelder verlangen könnte. Ausschluß wäre technisch möglich, wirtschaftlich aber wenig sinnvoll, wenn bspw. die durch die Eintrittsgelder erzielten Einnahmen geringer wären als die Kosten für die Umzäunung und das Arbeitsentgelt des Kassierers.

Die Folge mangelnder Ausschlußmöglichkeit besteht darin, daß das Auktionssystem des Marktes zusammenbricht. Warum auch sollte ein beliebiges Wirtschaftssubjekt seine Präferenzen für ein bestimmtes Gut offenbaren, wenn es genau weiß, daß es vom Konsum ohnehin nicht ausgeschlossen werden kann. Es ist für das Individuum wesentlich vorteilhafter, sich als Trittbrettfahrer zu verhalten und von der Nachfrage anderer zu profitieren. Nehmen nun alle Wirtschaftssubjekte diese Haltung ein, wird sich

\footnotetext{
202In Anlehnung an Vilfredo Pareto spricht man dann von einer pareto-optimalen Situation, wenn es nicht mehr möglich ist, ein bestimmtes Wirtschaftssubjekt besserzustellen, ohne ein anderes gleichzeitig schlechter zu stellen. Zur Ableitung pareto-optimaler Zustände, vgl. u.a. Musgrave, 1987, S. 70 ff., zur Kritik an der Paretianischen Bedingung als Kriterium allokativer Effizienz, vgl. Arnold, 1992, S. 32 f. ${ }^{203} \mathrm{Vgl}$. Musgrave, 1987, S. $60 \mathrm{ff}$.

${ }^{204}$ Als Beispiel dient u.a. die äußere Sicherheit. Vgl. Stiglitz/ Schönfelder, 1989, S. 114.
} 
keine effektive Marktnachfrage entwickeln und ein derart charakterisiertes Gut wird nicht privatwirtschaftlich angeboten werden. Eine alternative Form der Bereitstellung wird erforderlich, die in solchen Fällen üblicherweise steuerfinanziert durch die öffentliche Hand erfolgt. 205

Rivalität im Konsum ist dann nicht gegeben, wenn ein bestimmtes Gut einer unendlich steigenden Anzahl von Konsumenten Nutzen stiften kann, ohne daß sich dabei der Nutzen eines einzelnen reduziert, also keinerlei Grenzkosten existieren. ${ }^{206}$ Wird in einer derartigen Situation ein Preis erhoben, so bleibt die Nachfrage jener Konsumenten, deren Zahlungsbereitschaft positiv ist, jedoch unterhalb dieses Preises liegt, unbefriedigt. Jeder erhobene Preis ist damit im Vergleich zum Nulltarif pareto-inferior, da er den Konsum einschränken wird, ohne hierdurch Faktoren freizusetzen, die an anderer Stelle der Volkswirtschaft zur Produktion anderer Güter eingesetzt werden können. Der pareto-optimale Preis entspricht grundsätzlich den Grenzkosten, sind diese jedoch gleich Null, dann sollte auch der Preis gleich Null sein. ${ }^{207}$ Ausschluß, selbst wenn rein technisch möglich, sollte aufgrund zu erwartender Ineffizienzen in Form pareto-inferiorer Mengen nicht angewandt werden. Optimal ist folglich ein Anbieter, der von der Möglichkeit zum Ausschluß keinen Gebrauch macht; ein abermals über Steuern finanziertes staatliches Angebot liegt nahe.

\subsubsection{Mischgüter und effiziente Allokation}

Die oben genannte Ausschlußproblematik ist keineswegs ein Problem prinzipieller, sondern vielmehr gradueller Natur. Dies bedeutet, daß die Nutzen bestimmter Güter aus mehreren Komponenten bestehen können, die zum Teil mit, zum Teil ohne Ausschlußmöglichkeiten versehen sind. Im Falle letzterer handelt es sich um die externen Effekte. Der Grad der Öffentlichkeit, der sich mittels einer Emissions- oder auch Spillover-quote $(\ddot{E})$ konkretisieren läßt, kann damit alle Werte zwischen $\mathrm{O}$ (privates Gut) und 1 (öfftl. Gut) annehmen. ${ }^{208}$

${ }^{205}$ Das gleichermaßen auftretende Problem der Präferenzenthüllung und damit die Frage nach der erwünschten Bereitstellungsmenge wird in Demokratien annäherungsweise mit dem Modell des Medianwählers gelöst.

${ }^{206}$ Bezüglich der Grenzkosten muß unterschieden werden zwischen den hier betrachteten Grenzkosten des Konsums und jenen der Bereitstellung. Erstere beziehen sich auf den Konsum durch ein zusätzliches Individuum und damit möglicherweise einhergehende Überfüllungs- oder Verdrängungskosten. Letztere hingegen konzentrieren sich auf Kapazitätserweiterungen und können sich von den Grenzkosten im Konsum unterscheiden. Vgl. Grossekettler, 1985, S. 217 f.

${ }^{207} \mathrm{Vgl}$. Musgrave, 1987, S. $62 \mathrm{f}$.

${ }^{208}$ Die Emissionsquote $\ddot{E}$ ist definiert als Quotient aus dem Absolutwert der externen Effekte und dem Absolutwert der Gesamteffekte. Vgl. Grossekettler, 1985, S. 224. 
Die Ineffizienz der Bereitstellung derartiger Mischgüter $(0<\ddot{E}<1)$ besteht darin, daß der für den Konsum eines derartigen Gutes erhobene Preis zu hoch und damit die effektiv nachgefragte Menge nach dem Mischgut suboptimal im Sinne einer zu geringen Nachfrage ist. Ähnlich wie im Fall des reinen öffentlichen Guts wird kein Nutzer der externen Effekte seine Präferenz enthüllen, da er vom Konsum dieses Teilgutes nicht ausgeschlossen werden kann. Die Realisierung der optimalen Menge erfordert damit eine Internalisierung der externen Effekte, was im Falle positiver externer Effekte durch eine staatliche Subvention des betreffenden Gutes erfolgen kann, deren Höhe sich an der Emissionsquote $\ddot{E}$ ausrichten sollte. ${ }^{209}$

Eine Einschränkung scheint angebracht. Auch die Internalisierung von externen Effekten verursacht Kosten, die letztlich zu Ineffizienzen führen können. ${ }^{210}$ Es kann folglich nicht Sinn und Zweck sein, jeglichen, auch noch so geringen externen Effekt internalisieren zu wollen. Vielmehr müssen Kosten und Erträge der Internalisierung verglichen werden, ${ }^{211}$ was letztlich dazu führen kann, daß nicht Pareto-Optimalität, sondern eine Second-Best-Lösung sinnvoller erscheint. ${ }^{212}$ Bonus weist auf die Gefahr einer Internalisierung jeglicher externer Effekte hin, die er in einer "Aushöhlung der sozialen Marktwirtschaft" und einer völligen Stillegung des Wirtschaftslebens sieht. ${ }^{213}$

"In der Tat erweisen sich Elemente des Phänomens öffentlicher Güter als allgegenwärtig, wenn man genau hinsieht. $<$ Rein private Güter $>$, bisher absolut der Regelfall und unerläßlich für das Funktionieren des Marktes, sind innerhalb des Güterspektrums unversehens auf ein knife edge of reality zusammengeschrumpft. Da der Markt versagt, wo immer er auf ein Element öffentlicher Güter stößt, bleibt er nirgends unangefochten, er sieht sich auf seinen ureigensten Domänen plötzlich zutiefst in Frage gestellt. Wer die Marktwirtschaft lebensfähig erhalten will, kann das daher nicht durch ständiges defensives Zurückweichen vor dem Phänomen öffentlicher Güter erreichen. Er muß ganz im Gegenteil seine Aufmerksamkeit auf dieses Phänomen konzentrieren und wird dann entdecken, daß es gerade die Allgemeinheit ist, die - vertreten durch den Staat - fortwährend die Bedingungen zerstört, unter

\footnotetext{
${ }^{209} \mathrm{Im}$ umgekehrten Fall negativer externer Effekte wäre der erhobene Preis zu gering und die effektiv nachgefragte Menge damit zu hoch. Der einzelne Konsument würde in dieser Situation nicht die vollen sozialen Kosten der Bereitstellung bezahlen. Der Staat müßte den Konsum eines mit externen Kosten verbundenen Gutes mit der Auferlegung von Steuern bestrafen, um somit eine geringere Bereitstellungsmenge zu gewährleisten.

${ }^{210}$ So sind mit der Aufbringung von Subventionsmitteln Transaktionskosten wie die Kosten der Informationsgewinnung zur Bestimmung der internalisierungsbedürftigen Externalität oder auch mögliche. mit der Steuererhebung einhergehende Verzerrungen verbunden, weshalb nicht ausgeschlossen werden kann, daß sich per Saldo ein allokativer Nachteil ergibt.

${ }^{211}$ Vgl. Brümmerhoff, 1990, S. 76 f.

${ }^{212}$ Zur Theorie des Zweitbesten, vgl. u.a. Davis/ Whinston, 1967, Millward, 1971, ch. 4 oder auch Külp, 1975, Kap. 4.1. und 4.2.

${ }^{213} \mathrm{Vgl}$. Bonus, 1979/80, S. $69 \mathrm{ff}$.
} 
denen der einzelne selbstverantwortlich handeln kann - statt sie herzustellen und zu verbessern." 214

Ebenso wie das Kriterium des Ausschlusses ist auch jenes der Rivalität im Konsum kein Problem prinzipieller, sondern gradueller Natur, wobei sich der Grad der Rivalität

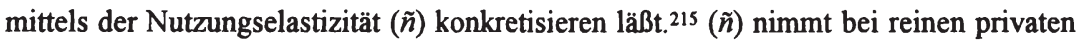
Gütern den Wert 1, bei reinen öffentlichen Gütern den Wert 0 an. Solche Güter, bei denen der individuelle Nutzen eine erst ab einem gewissen Punkt fallende Funktion der Anzahl der Konsumenten ist, $(0<\tilde{n}<1)$ werden in Anlehnung an den Begründer der Klubtheorie, James M. Buchanan, als unreine öffentliche Güter oder Klubgüter bezeichnet.

"The interesting cases are those goods and services, the consumption of which involves some "publicness", where the optimal sharing group is more than one person or family but smaller than an infinitely large number. The range of "publicness" is finite." 216

Buchanan argumentiert in seiner Untersuchung bezüglich der allokativen Effizienz bei der Bereitstellung von Klubgütern mit dem Beispiel eines Schwimmbades, in dem zusätzliche Besucher bis zum Erreichen einer Kapazitätsgrenze kaum fühlbare Grenzkosten verursachen. ${ }^{217}$ Wird diese Kapazitätsgrenze erreicht und weiteren Nachfragern dennoch Zutritt gewährt, dann entstehen fühlbare Grenzkosten in Form von qualitativen Einbußen (Überfüllungskosten) ${ }^{218}$

Liegt ein derartiges Gut mit begrenzten Vorteilen der gemeinsamen Nutzung vor, dann wird ein Anbieter, der einen Preis in Höhe der nunmehr vorhandenen, aber bis zum Erreichen der Kapazitätsgrenze kaum fühlbaren Grenzkosten erhebt, Verluste hinnehmen müssen. Schließlich verursacht die Bereitstellung von Klubgütern ex definitione Gesamtkosten, die über die Grenzkosten hinausgehen. Folglich könnte der Anbieter dazu neigen, einen Preis in Höhe der Durchschnittskosten zu erheben, um seine gesamten Kosten zu decken. Dieser Preis wiederum ist - wie weiter oben gezeigt - ineffizient.219

${ }^{214}$ Bonus, 1979/80, S. 69/70. Der Begriff des knife-edge of reality geht auf Samuelson zurück. Vgl. Samuelson, 1972, S. 503.

${ }^{215}(\tilde{n})$ kann als partielle Elastizität der erforderlichen Angebotsmenge in bezug auf zusätzliche Nutzer definiert werden. Vgl. Grossekettler, 1985, S. 217.

${ }^{216}$ Buchanan, 1965, S. 2.

${ }^{217} \mathrm{Vgl}$. ebd. S. I ff.

${ }^{218} \mathrm{Zu}$ alternativen Reaktionsmöglichkeiten beim Erreichen der Kapazitätsgrenze, vgl. Grossekettler, 1985, S. 216.

${ }^{219}$ So bliebe die Nachfrage eines potentiellen Konsumenten, dessen Zahlungsbereitschaft geringer als der erhobene Durchschnittskostenpreis ist, unberücksichtigt. Und dies, obwohl seine Zahlungsbereitschaft sehr wohl größer sein kann als die Grenzkosten. 
Es stellt sich folglich die Frage nach der effizienten Angebots- und Preisform für Klubgüter. Die ökonomische Theorie der Klubs vermag hierzu Antworten zu geben. ${ }^{220}$ So wird u.a. im Zusammenhang mit der Frage nach der Selbstfinanzierung des Klubgutes auf die Möglichkeit der Erhebung eines two-part-tariffs hingewiesen, der zusätzlich zu einer mengenabhängigen Nutzungsgebühr (Toll) eine fixe Mitgliedschaftsgebühr beinhaltet. Letztere deckt den zur vollständigen Selbstfinanzierung verbleibenden Teil der Kosten. 221

Trotz des Postulats der Grenzkostenpreisbildung222 werden auch Möglichkeiten einer von den Grenzkosten abweichenden mengenabhängigen Gebühr diskutiert. So argumentieren bspw. NG und Weisser, mengenunabhängige Mitgliedschaftsgebühren können u. U. so hoch sein, daß dadurch potentielle Nachfrager aus dem Markt gedrängt werden könnten. 223 Unter verschiedenen Prämissen leiten NG/ Weisser "optimale twopart-tariff-Theoreme" ab, die je nach Höhe der Preiselastizitäten und der Verbrauchsgewohnheiten verschiedener Nutzer unterschiedlich hohe Tolls vorsehen. Neben der Option einer den Grenzkosten entsprechenden Toll kann selbige die Grenzkosten auch übersteigen, um darnit die mengenunabhängige Mitgliedschaftsgebühr zu reduzieren. Schließlich ist auch eine Toll denkbar, die die Grenzkosten unterschreitet, was letztlich zu einer höheren Mitgliedschaftsgebühr führt.

${ }^{220}$ Das erste allgemeine Modell für unreine öffentliche Güter geht auf Buchanan zurück, der 1965 unter allerdings restriktiven Annahmen die Bedingungen für eine effiziente Allokation von Klubgütern herleitete. Vgl. Buchanan, 1965, S. 1 ff. Einen guten Überblick über spätere Klubmodelle, die u.a. heterogene Mitgliedschaften und eine variable Nutzung des Klubgutes erlauben sowie eine gesamtwirtschaftliche Perspektive einnehmen, geben Sandler/ Tschirhart, 1980, S. $1487 \mathrm{ff}$.

${ }^{221}$ Wenngleich die beiden Kriterien Ausschlußmöglichkeit und Rivalität im Konsum voneinander getrennt behandelt werden sollen, erscheint an dieser Stelle folgende Frage angebracht: Inwieweit stellt die Klubgründung selbst ein öffentliches Gut dar, m. a. W., ist die Klubgründung unabhängig jeglicher externer Effekte des vom betreffenden Klub angebotenen Klubgutes forderungswürdig? Grossekettler bemerkt diesbezüglich. daß die Gründung von Vereinen als solche ein Kollektivgut sei, da ein Gründer andere von den Gründungsvorteilen um der Sache selbst willen nicht ausschließen könne. Vgl. Grossekettler, 1985, S. 237. Unterstellt man also bspw. ein Gut mit begrenzten Vorteilen kollektiver Nutzung und einem Fehlen jeglicher externer Effekte, dann führt der Verzicht der Gründungsunterstützung aufgrund der externen Effekte der Gründung zu Klubgründungen in suboptimaler Menge. Eine einmalige Subventionierung der Klubgründung - nicht des betreffenden Gutes - scheint unter diesem Aspekt denkbar, wobei allerdings wiederum die Ineffizienzen der unterlassenen Klubgründung mit jenen der Gründungsunterstützung verglichen werden müßten.

${ }^{222}$ Vgl. u.a. Coase, 1946, S. 173, Margolis, 1957, S. 452 und Sherman, 1967, S. 237 f.

${ }^{223}$ Dieses Problem stellt sich insbesondere dann, wenn von der Annahme variabler Nutzung ausgegangen wird, d.h. ein potentieller Konsument ein bestimmtes Klubgut vielleicht seltener nutzen möchte als andere Nutzer. Es sei keineswegs sicher, so die Argumentation von NG und Weisser, daß der gleichwohl negative Preiseffekt einer die Grenzkosten übersteigenden Toll größer sei als der damit einhergehende positive Effekt in Form der Reduzierung der aus dem Markt gedrängten Konsumenten. Vgl.NG/ Weisser, 1974, S. $337 \mathrm{f}$. 
Auf eine weitere Möglichkeit verweist bspw. Lewis bereits 1941, indem er die persönliche Preisdifferenzierung als Mittel aufzeigt, um den für manche Konsumenten prohibitiven Charakter einer zu hohen Mitgliedschaftsgebühr zu umgehen. ${ }^{224}$ Berglas (1976) versucht schließlich nachzuweisen, daß Klubgüter unter bestimmten, allerdings restriktiven Voraussetzungen mit derselben allokativen Effizienz auch von wettbewerblichen Unternehmen bereitgestellt werden können. 225

Die folgenden Abschnitte werden sich der Frage zuwenden, inwieweit es sich im Falle von Sportgütern um öffentliche Güter im oben beschriebenen Sinne handelt. Damit einhergehend wird untersucht, inwieweit sich die Praxis öffentlicher Sportförderung wie auch die Angebotsstruktur von Sportgütern mit den dargestellten neoklassischen Überlegungen erklären läßt.

\subsection{Sport - ein öffentliches Gut?}

\subsubsection{Zur Spezifizierung des Begriffes Sport im Lichte der Kollektivgüter- theorie}

Sport ist kein homogenes Gut, sondern eher der Oberbegriff einer Reihe von Gütern, die durchaus unterschiedliche Eigenschaften haben können.

Im Zusammenhang mit der hier relevanten Frage nach möglichen externen Effekten und Vorteilen der kollektiven Nutzung von Sportgütern sind insbesondere drei Kriterien von Bedeutung: die Art der Nutzung von Sportstätten, die Unterscheidung verschiedener Sportarten sowie verschiedene Angebotsformen zur Sportausübung. ${ }^{226}$

Das erste Kriterium unterscheidet de facto zwischen aktiver Sportausübung und passivem Sportkonsum, womit einerseits eher freizeit- und breitensportspezifische Sportgüter, ${ }^{227}$ andererseits hingegen der Spitzen- und damit in aller Regel auch der Zuschauersport angesprochen werden.

\footnotetext{
${ }^{224}$ Dies führt selbstverständlich dazu, das betreffende Gut einigen Konsumenten unter Kosten anzubieten und den Verlust durch andere zu decken. Vgl. Lewis, 1941, S. $267 \mathrm{ff}$.

${ }^{225}$ Die essentielle Voraussetzung im Berglas-Modell ist die im Verhältnis zum gesamten Markt geringe Größe des optimalen Unternehmens. Vgl. Berglas, 1976, S. 117 - 121.

${ }^{226}$ Vergleichbare Diskussionen über den Charakter des Gutes Sport finden sich - allerdings nur ansatzweise - bei Gärtner, 1988, S. 44/45, Grossekettler, 1985, S. 211 ff. und Heinemann, 1984, S. 28 ff., 1987 b, S. 236/237 sowie 1988, S. 70/71. Darüber hinaus wird in verschiedenen Veröffentlichungen zur ökonomischen Theorie des Klubs anhand von Schwimmbädern argumentiert. Vgl. u.a. Buchanan, 1965, S. $1 \mathrm{ff}$.

${ }^{227}$ Der Begriff Breitensport ist definiert als der vorwiegend in Vereinen ausgeübte wettkampforientierte Sport, der Terminus Freizeitsport meint nicht wettkampforientiert ausgeübte Formen des Sports. Vgl. Blank, 1983, S. 177.
} 
Sport läßt sich zum anderen trennen in die große und steigende Anzahl verschiedener Sportarten. Diese unterscheiden sich je nach der Anzahl der teilnehmenden Sportler in Mannschafts- und Individualsportarten, nach dem geographischen Ort des Sporttreibens in Freiluft- und Hallensportarten, nach der Frage des Körperkontakts in Sportarten mit und ohne Körperkontakt sowie sportstättenbezogen in Kern- und Spezialsportarten.

Schließlich kann Sport sowohl in organisierter Form in Vereinen, in unorganisierter Form in kommerziell geführten Sportstätten als auch unter dem Dach einiger weiterer, oben beschriebener Anbieter ausgeübt werden. ${ }^{228}$

Abbildung 26: Zur Präzisierung des Begriffes Sport

\begin{tabular}{|c|c|c|}
\hline \multicolumn{2}{|l|}{ Kriterien } & Präzisierung \\
\hline \multirow{4}{*}{$\begin{array}{l}\text { Art der Nutzung der } \\
\text { Sportgüter }\end{array}$} & \multirow[t]{3}{*}{ Sport treiben } & Freizeitsport (nicht wettkampforientiert) \\
\hline & & Breitensport (wettkampforientiert) \\
\hline & & Berufssport, Spitzensport \\
\hline & Sport konsumieren & Zuschauersport \\
\hline \multirow{8}{*}{$\begin{array}{l}\text { Sportartenunterschei- } \\
\text { dung nach }\end{array}$} & \multirow[t]{2}{*}{ Anzahl der Teilnehmer } & Mannschaftsportarten \\
\hline & & Individualsportarten \\
\hline & \multirow[t]{2}{*}{ Ort } & Freiluftsportarten \\
\hline & & Hallensportarten \\
\hline & \multirow[t]{2}{*}{ Körperkontakt } & Mit Körperkontakt \\
\hline & & Ohne Körperkontakt \\
\hline & \multirow[t]{2}{*}{ Erforderliche Sportstätten } & $\begin{array}{l}\text { Kernsportstätten } \Rightarrow \text { Sportplätze u. -hal- } \\
\text { len (Turnen, Fußball, Leichtathletik etc.) }\end{array}$ \\
\hline & & $\begin{array}{l}\text { Sondersportanlagen } \Rightarrow \text { für Tennis, Rei- } \\
\text { ten, Golf, Schießen etc.) }\end{array}$ \\
\hline \multirow{2}{*}{\multicolumn{2}{|c|}{ Personenbezogene Unterscheidungen }} & Jugend-, Erwachsenen-, Seniorensport \\
\hline & & Versehrten-, Nichtversehrtensport \\
\hline \multirow{4}{*}{\multicolumn{2}{|c|}{$\begin{array}{l}\text { Sportanbieterbezogene Unterscheidung des Sport- } \\
\text { treibens }\end{array}$}} & Vereinssport \\
\hline & & Kommerzieller Sport \\
\hline & & Schul- und Hochschulsport \\
\hline & & weitere Angebotsformen des Sports \\
\hline
\end{tabular}

${ }^{228}$ Weitere mögliche Abgrenzungen des Sportbegriffs sind denkbar, so z.B. eine Unterscheidung zwischen kostenintensiven und kostengünstigen Sportstätten. Zur Gruppe der kostenintensiven zählen zweifelsfrei Großstadien oder Badeanstalten, als kostengünstig sind hingegen "Bolzplätze", Trimm-DichPfade und ähnliches anzusehen. 
Die folgenden Ausführungen behandeln verschiedene allokationstheoretisch relevante Aspekte des Gutes Sport. Zum Abschluß der Diskussion wird hinterfragt, inwieweit die am Ende von Kapitel III zusammengefaßten erläuterungsbedürftigen Tatbestände erklärt werden können.

\subsubsection{Sportgüter und die Möglichkeit zum AusschluB - Internalisierte Nut- zen vs. externe Effekte des Sports}

\subsubsection{Kernnutzen von Sportgütern}

Neben den in Abbildung 26 dargestellten Möglichkeiten einer weiteren Präzisierung des Sportbegriffs muß auch der Nutzen, den Individuen aus der Nachfrage nach Sportgütern erzielen, differenziert betrachtet werden.

Nur auf diese Weise kann die Frage, inwieweit die Theorie der öffentlichen Güter die Bereitstellung öffentlicher Gelder und deren Umfang erklären kann, ausreichend beantwortet werden.

Der Sport, das kann leicht gezeigt werden, ist kein reines öffentliches Gut. Welche Spezifizierung des Sportbegriffs man auch immer heranzieht, ein Ausschluß vom Primärgut Sporttreiben bzw. der Nutzung von Sportstätten scheint in den meisten Fällen möglich.

Das Recht zum Besuch einer Sportanlage, unabhängig vom expliziten $Z$ weck dieser Nutzung (sei es zum Zwecke des Sporttreibens wie auch des Zuschauens etc.), kann an die Entrichtung einer Gebühr gekoppelt werden, Ausschluß vom Primär- oder Kernnutzen des Sportgutes ist also technisch grundsätzlich möglich.

Bezüglich der wirtschaftlichen Vertretbarkeit des Ausschlusses haben die theoretischen Ausführungen gezeigt, da $B$ die Kosten des Ausschlusses mit den Kosten der Bereitstellung $\mathrm{zu}$ vergleichen sind. Auch diesbezüglich kann festgehalten werden, daß es sich nicht um ein reines öffentliches Gut handelt; Ausschluß ist vielmehr im allgemeinen wirtschaftlich vertretbar.

Ausnahmen, in denen die Kosten des Ausschlusses durch Umzäunen einer Anlage und Beschäftigung eines Kassierers in keinem Verhältnis zu den Kosten der Bereitstellung stehen, stellen z.B. ein Trimm-Dich-Pfad oder ein "Bolzplatz" dar, Sportanlagen also, die keine größeren laufenden Instandhaltungsmaßnahmen oder höheren Energieverbrauch bedingen. In diesen wenigen Fällen erscheint eine entgeltlose öffentliche Bereitstellung aus allokationstheoretischer Sicht begrüßenswert. 
Die aus dem Besuch von Sportstätten letztlich resultierenden internalisierten, hier als Kernnutzen bezeichneten, Nutzen sind von Individuum zu Individuum verschieden.

Für die einen bestehen sie in der Freude an der körperlichen Ertüchtigung. Für die anderen stellt der Sport ein Mittel zum Zwecke des Knüpfens sozialer Kontakte, der Erlangung von Ansehen, der Gesundheitserhaltung oder -wiedergewinnung etc. dar. Internalisierte Nutzen aus dem "passiven" Live-Konsum von Zuschauer- oder Spitzensport bestehen in erster Linie im Erleben von Spitzenleistungen.

Es kann damit festgehalten werden: Sportgüter sind keine reinen öffentlichen Güter im Sinne fehlender Ausschlußmöglichkeiten in bezug auf den Kernnutzen, da ein Ausschluß im allgemeinen sowohl technisch möglich als auch wirtschaftlich sinnvoll erscheint.

Daneben können mit dem Sport weitere, hier als Nebennutzen bezeichnete Nutzen einhergehen, die möglicherweise nicht internalisiert sind und damit externe Effekte darstellen.

Die theoretischen Erörterungen haben gezeigt, daß das Vorhandensein externer Effekte eine öffentliche Subventionierung erfordern kann, um die "optimale" Versorgung mit dem betreffenden Gut zu gewährleisten.

Im Zusammenhang mit dem Sport werden mitunter - allerdings sehr pauschal und ohne wissenschaftliche Fundierung - positive Auswirkungen auf Gesundheit, Sozialverhalten Gesellschaft und Wirtschaftsförderung sowie die Funktion des Sports als Mittel zur Repräsentation hervorgehoben, um die dargestellte öffentliche Subventionierung zu rechtfertigen. ${ }^{229}$

Diese möglichen externen Effekte des Sports werden im weiteren Verlauf etwas näher beleuchtet.

\subsubsection{Externe Effekte des Sports}

\subsection{Sport und Gesundheit}

Aussagen zu positiven Auswirkungen des Sports auf die Gesundheit lassen sich in sportpolitischen Schriften zur Genüge finden.230

Bevor innerhalb dieses Abschnitts die Richtigkeit dieser Hypothese diskutiert wird, soll eingangs die Frage gestellt werden, inwieweit potentielle positive Gesundheitswirkungen des Sports grundsätzlich als externe Effekte anzusehen sind.

\footnotetext{
${ }^{229} \mathrm{Vgl}$. u.a. Bundesministerium des Innern, 1991, S. 14 f., Deutscher Sportbund, 1990, S. 26 f., Heinemann, 1987b, S. 236.

${ }^{230} \mathrm{Vgl}$. Deutscher Sportbund, 1991, S. 33. Auch ein Großteil kommunaler Sportförderrichtlinien beginnt mit der allgemeinen Begründung kommunaler Sportpolitik durch positive Gesundheitswirkungen des Sports.
} 
Zunächst kann festgehalten werden, daß ein Mensch, der aufgrund seines Verhaltens irgend etwas für seine Gesundheit tut, primär einmal sich selbst nutzt. Umgekehrt leidet ein Individuum, das infolge seines Verhaltens seiner Gesundheit schadet, selbst am meisten darunter. Schließlich - wollte man unterstellen, daß der Sport auch gesundheitsschädigend sein kann - handelt es sich dabei nicht um irgendwelche Ansteckungskrankheiten, die dann unter Umständen auch nichtsporttreibende Individuen befallen könnten.231 Potentielle positive oder auch negative Auswirkungen des Sports auf die Gesundheit sind damit allererst einmal interne Effekte.

Nun darf das Gut Gesundheit allerdings nicht mit anderen in einen Topf geworfen werden. Moderne Gesundheitssysteme zeichnen sich in der Regel dadurch aus, daß dem einzelnen Individuum durch ein Krankenversicherungssystem zumindest teilweise das Risiko genommen wird, neben den ohnehin schon hohen immateriellen Kosten einer Krankheit zusätzliche monetäre Kosten bspw. in Form von Einkommensausfall etc. ertragen zu müssen.

Versicherungen generell und damit auch Krankenversicherungen haben den Zweck, das Individuum vor unvorhersehbaren Risiken zu schützen bzw. die möglicherweise für den einzelnen immensen Kosten einer Krankheit auf die Dauer der Beitragszahlung oder auch auf die Allgemeinheit zu verteilen.

Nun kann man das Krankenversicherungssystem aber wiederum nicht mit anderen Gütern, die im allgemeinen versichert werden, vergleichen. Viele der Güter, die in aller Regel versichert werden, zeichnen sich dadurch aus, daß sich die Prämie für das zu versichernde Gut nach dem Risiko einer eventuell erfolgenden Inanspruchnahme einer Versicherungsleistung richtet. Inhaber von Cabriolets bezahlen aufgrund des höheren Diebstahlrisikos höhere Kaskobeträge, Stuntmen bezahlen höhere Prämien für Lebensversicherungen usw.

Das gesetzliche Gesundheitssystem in der Bundesrepublik Deutschland kennt eine derartige Verknüpfung von Wahrscheinlichkeit einer Leistungsinanspruchnahme und der vom Versicherten zu entrichtenden Prämie zumindest bislang noch nicht. ${ }^{232}$ Die zu entrichtende Versicherungsprämie ist bis zu einer Maximalgrenze ${ }^{233}$ lediglich vom Bruttoeinkommen des Versicherten abhängig. Mögliche Auswirkungen bestimmter Le-

${ }^{231}$ In der Literatur finden sich zur Erläuterung externer Effekte zuweilen Beispiele wie jenes der Impfung, die andere Individuen vor dem Befall von Ansteckungskrankheiten bewahrt. Vgl. Musgrave, 1987, S. 90 .

${ }^{232}$ Bezüglich des privaten Versicherungssystems ist einschränkend hinzuzufügen, daß sich die Prämie im Gegensatz zur gesetzlichen Krankenversicherung nicht nach dem Einkommen, sondern primär nach dem Eintrittsalter richtet.

${ }^{233}$ Diese Beitragsobergrenze beträgt derzeit (Stand 1994) 5.700,- DM. Bis zu diesem Betrag müssen je nach Versicherung zwischen etwa 6 - 6,5\% des Bruttoeinkommens sowohl vom Arbeitnehmer als auch vom Arbeitgeber an die jeweilige Krankenkasse bezahlt werden. 
bensweisen auf die Gesundheit werden damit zumindest teilweise allererst externalisiert, externe Effekte damit von politischer Seite geschaffen.

Dabei wäre es durchaus denkbar, nachgewiesene Kosteneinsparungen (Zusatzkosten) mittels geringerer (höherer) Beiträge zu belohnen (bestrafen) und damit das Entstehen externer Effekte zu verhindern. ${ }^{234}$ Schließlich müßte eine derartige Kalkulation doch im Sinne des Versicherers wie auch zumindest jenes Versicherten liegen, der eine Beitragsreduzierung zu erwarten hätte.

Interessanterweise tendieren Pressemeldungen, die diesbezüglich Stellung nehmen, mitunter in Richtung negativer Gesundheitsauswirkungen des Sports und berichten von Diskussionen über mögliche Zusatzversicherungen für Sportler. ${ }^{235}$ Darüber hinaus zeigen auch verschiedene ausländische Krankenversicherungssysteme keinerlei Anhaltspunkte in Richtung einer nach sportlicher Aktivität differenzierenden Beitragshöhe. 236

Nichtsdestotrotz, das derzeitige gesetzliche Krankenversicherungssystem in der BRD sieht keine unterschiedliche Behandlung verschiedener Kundengruppen vor, womit Auswirkungen des Sports auf die Gesundheit, seien diese positiv oder auch negativ, zumindest teilweise tatsächlich zu externen Effekten werden.

Zurück zur eingangs aufgeführten These, der Sport erhalte die Gesundheit und sei damit subventionierungswürdig. Im Rahmen dieser Betrachtung muß zunächst einmal zwischen aktiver Sportausübung und passivem Sportkonsum, dem Zuschauersport differenziert werden. Letzterer muß ausgeschlossen werden, der Sport kann positive Gesundheitswirkungen immer nur für die Sporttreibenden bedingen. Daneben müssen auch weite Bereiche des Spitzensports ausgenommen werden, was unter anderem angesichts des in vielen Sportarten gängigen Dopingmißbrauchs oder körperlicher Verschleißerscheinungen aufgrund zu hoher Belastungen nachvollzogen werden kann. Potentielle Gesundheitswirkungen des Sports beschränken sich damit primär auf jene Sportgüter, die zum Zwecke des freizeit- und breitensportorientierten Sporttreibens bereitgestellt werden.

\footnotetext{
${ }^{234}$ So müßte es zumindest in gewissen Grenzen möglich sein, körperliche Fitneß und sportliche Aktivität (abgesehen von der reinen Mitgliedschaft in Vereinen, die nicht zwangsläufig mit sportlicher Tätigkeit einhergehen muß) zu überprüfen. Denkbar wäre bspw. ein erforderlicher Nachweis über das Ablegen des Deutschen Sportabzeichens.

${ }^{235}$ Vgl. u.a. Die ZEIT, vom 19.7.1985, "Gesundheit - ein Luxus?"

${ }^{236}$ Betrachtet wurden die Krankenversicherungssysteme in den EG-Ländern. Die Systeme ähneln dem deutschen größtenteils. In 10 Ländern erfolgt die Finanzierung durch Arbeitnehmer und -geber mittels unterschiedlich hoher Prozentsätze des Bruttoeinkommens. In einigen Ländem werden zusätzliche Zuschüsse vom Staat gewährt. Lediglich Dänemark, das sein staatliches Krankenversicherungssystem aus Steuermitteln finanziert sowie England, welches altersabhängige Faktoren berücksichtigt. weichen von der mehrheitlichen Praxis ab. Vgl. Bundesministerium für Arbeit und Sozialordnung, 1993
} 
Ohnehin ist die oben genannte These, so gerne sie auch von seiten der Sportpolitik aufgeführt wird, in dieser pauschalen Art und Weise nicht zu halten. Eine etwas differenziertere Betrachtung potentieller Gesundheitswirkungen des Sports ist erforderlich, wozu auf Erkenntnisse der Medizin, insbesondere der Sportmedizin zurückgegriffen wird.

Zunächst zu möglichen Auswirkungen des Sports auf das Herz-Kreislauf-System: Dieser Aspekt ist von ganz besonderer Bedeutung, da mehr als eine halbe Million Menschen allein in der alten BRD jährlich einen Herzinfarkt erleiden; etwa ein Drittel dieser Menschen stirbt im ersten Jahr. Krankheiten des Kreislaufsystems verursachen Gieseler zufolge einen Ausfall von fast 30 Millionen Arbeitstagen pro Jahr und die Folgekosten des Bewegungsmangels belaufen sich auf "60 Milliarden DM".237 Darüber hinaus scheiden sehr viele Arbeitnehmer etliche Jahre vor dem eigentlichen Rentenalter aus dem Erwerbsleben aus.

Von seiten der Medizin wird zwar kaum ernsthaft bestritten, daß der Sport in einer zunehmend bewegungsarmen Umwelt einen positiven Einfluß auf das Herz-KreislaufSystem des Menschen hat, es läßt sich jedoch keineswegs mit Sicherheit sagen, wie stark dieser Einfluß ist und wieviel Gelder die Krankenversicherungen (und damit im Falle des deutschen Krankenversicherungssystems die Allgemeinheit) durch sporttreibende Individuen einsparen.

Sportmediziner sind unterschiedlicher Ansicht. Mellerowicz bspw. beziffert den potentiellen volksgesundheitlichen Wert von Ausdauertraining und - wohlgemerkt - gesunder Lebensführung auf mehr als 50 MRD DM jährlich, ohne diese Zahlen allerdings näher herzuleiten. 238

Beuker oder auch Moser widersprechen positiven Effekten zwar nicht grundsätzlich, verweisen aber darauf, daß präventive Wirkungen sportlichen Trainings nur sehr schwer eindeutig nachzuweisen sind. ${ }^{239}$ Letzterer sieht geringere Herzinfarktraten in Japan oder auch in den USA vorrangig in einer ansonsten gesünderen Lebensweise und nicht primär in einer sportlicheren begründet. 240

\footnotetext{
${ }^{237} \mathrm{Vgl}$. Gieseler 1983, S. 51. Bezüglich der Frage, inwieweit die daraus resultierenden Krankheitskosten unmittelbar dem Bewegungsmangel zuzuschreiben sind, besteht allerdings Uneinigkeit. Vgl. hierzu u.a. Rost, 1989, S. 79 und 1989 a, S. 292 f. sowie Jung/ Ulmer, 1983, S. 62 ff.

${ }^{238} \mathrm{Vgl}$. Mellerowicz, 1984, S. 4.

${ }^{239} \mathrm{Vgl}$. Beuker, 1986, S. 44.

${ }^{240} \mathrm{Vgl}$. Moser, 1989, S. 221 f. Eine ähnliche Ansicht vertreten Jung und Ulmer, die in der Proklamation des Sports als Mittel zur Prävention von Herz-Kreislauf-Krankheiten lediglich eine Ablenkung von den Hauptproblemen sehen, welche ihrer Ansicht nach im Nikotinabusus, sowie der Fettsucht liegen.
} 
Empirische Untersuchungen auf diesem Gebiet zeigen folgendes: Brunner (1960), Taylor (1962) und Morris (1966) konnten innerhalb empirischer Studien bezüglich der Gefahr einer Herz-Kreislauf-Erkrankung bei körperlich Berufstätigen sowie Angestellten zeigen, daß die körperlich Tätigen einem geringeren Risiko ausgesetzt waren und führten dies auf die körperliche Tätigkeit zurück. Stamler (1960) hingegen kam anhand einer Studie über Arbeiter einer Gasgesellschaft in Chicago zu gegenteiligen Ergebnissen, was er wiederum auf die risikointensivere Lebensweise der Arbeiter zurückführte. Eine eindeutige Stellungnahme läßt sich aufgrund dieser Studien folglich nicht ableiten. Es erscheint vielmehr wahrscheinlich, daß beide Faktoren, Bewegung und auch sonstige Lebensweise ${ }^{241}$ einen Einfluß haben und daß lediglich eine separate Betrachtung beider Faktoren vernünftige Aussagen erlauben kann.

Derartige Aussagen wurden durch zwei getrennte, von Morris und Pfaffenberger durchgeführte Langzeitstudien ermöglicht, die sich nicht der Bewegung innerhalb des Berufslebens, sondern jener innerhalb der Freizeit widmeten und die darüber hinaus isoliert sonstige Risikofaktoren betrachteten. ${ }^{242}$

Morris kam innerhalb seiner die Sport- und Rauchgewohnheiten von Londoner Angestellten betrachtenden Studie zu dem Ergebnis, daß sportliche Aktivität unabhängig von den jeweiligen Risikofaktorkonstellationen eine präventive Wirkung gegenüber Herz-Kreislauf-Krankheiten mit sich bringt.

Tabelle 21: Herz-Kreislauf-Ersterkrankung in 10 Jahren

\begin{tabular}{|l|l|l|l|}
\hline \multicolumn{2}{|l|}{} & Sportler & Nichtsportler \\
\hline \multicolumn{2}{|l|}{ Nichtraucher } & $1,5 \%$ & $3,8 \%$ \\
\hline \multirow{2}{*}{ Zigarettenraucher } & $11-20$ täglich & $4,6 \%$ & $9,6 \%$ \\
\cline { 2 - 5 } & 21 und mehr & $4,6 \%$ & $11,6 \%$ \\
\hline
\end{tabular}

Untersuchungspersonen, $n=17.944$

Quelle: Morris, J., abgedruckt in: Deutsche Zeitschrift für Sportmedizin, 40 (1989), Nr. 6, S. 223.

Gleichwohl wird darauf verwiesen, daß diese Schutzwirkung bei Rauchern wesentlich geringer ist und die Frage Raucher oder Nichtraucher letztlich größeren Stellenwert einnimmt. Zum anderen treten bei Rauchern während des Sporttreibens deutlich überdurchschnittlich kardiovaskuläre Zwischenfälle auf. ${ }^{243}$

\footnotetext{
${ }^{241}$ Hierzu gehören neben der oben genannten Fettsucht und dem Nikotinabusus auch psychische Belastungen, sonstige Ernährungsgewohnheiten sowie der Konsum von Alkohol etc.

${ }^{242}$ Vgl. Morris, 1980, S. 1207 und Pfaffenberger, 1978, S. 161.

${ }^{243}$ Es wäre interessant zu wissen, inwieweit öffentlich geförderte Sportvereinsmitglieder Raucher oder Nichtraucher sind, um damit weitere Aussagen ableiten zu können. Zahlen hierzu sind leider nicht bekannt.
} 
Als Fazit bezüglich des Zusammenhangs zwischen sportlicher Aktivität und HerzKreislauf-Erkrankungen soll folgendes festgehalten werden: Sportliche Aktivität kann, von anderen Faktoren losgelöst betrachtet, das Risiko einer Erkrankung verringern, wenngleich die beste Prävention wohl in der Kombination mit einer auch ansonsten gesunden Lebensweise zu sehen ist. Der Verweis einiger Kritiker darauf, daß es vorrangig Sportler sind, die sich durch eine auch ansonsten gesunde Lebensweise auszeichnen, kann dieses Argument entkräften, nicht jedoch widerlegen. Eine fundierte Aussage über die Höhe möglicher Einsparungen für das Gesundheitssystem läßt sich allerdings nicht ableiten.

Neben diesen positiven Auswirkungen des Sporttreibens auf das Herz-Kreislauf-System birgt der Sport aber auch gewisse Gefahren in sich. Nicht nur im Spitzensport entstehen immer wieder mehr oder weniger schwere Verletzungen, vorrangig des Bewegungsapparates. Der Sport kann folglich auch negative externe Effekte verursachen. Empirische Untersuchungen belegen, daß Sportverletzungen in erster Linie in den Sportarten Fußball, Hockey, Eishockey und Handball sowie in der "körperlosen" Sportart Basketball auftreten. ${ }^{244}$ Einschränkend muß allerdings angefügt werden, daß es sich dabei zumeist um leichtere Verletzungen ohne Folgen handelt. ${ }^{245}$ So bestehen bspw. 95\% der Fußballverletzungen aus Prellungen und leichteren Zerrungen.

Sollen Aussagen über die Gefährlichkeit verschiedener Sportarten getroffen werden, dann dienen diese Statistiken der Verletzungszahlen wenig, da sie Verletzungen aller Schweregrade enthalten. Fragt man hingegen nach der Dauer der ärztlichen Behandlung oder nach der verbleibenden Invalidität, dann wird das Feld nicht mehr von den o.g. Sportarten, sondern vom Reit- und Skisport angeführt. ${ }^{246}$ Todesfälle treten statistisch gesehen am ehesten beim Reiten, Tauchen und beim Flugsport auf.

Frey (1991) lehnt Sportarten mit Schnellkraftbewegungen wie abruptem Krafteinsatz (z.B. Tennis), Schnellkraftbewegungen mit Niedersprüngen (bspw. Weitsprung), maximalen Kraftbelastungen (z.B. Gewichtheben), statischen Beanspruchungen, Gegnerkontakt sowie mit hoher Fortbewegungsgeschwindigkeit (z.B. Skilaufen) unter gesundheitlichen Aspekten grundsätzlich ab. ${ }^{247}$ Als gesundheitsfördernd betrachtet er hingegen Sportarten mit aerobem Ausdauertraining (als Präventivmaßnahme gegen HK-Erkrankungen), dynamisches Krafttraining, Dehn-sowie Beweglichkeitsübungen. In diesem Zusammenhang nennt er vor allem das Laufen, Skilanglaufen, Rudern, Radfahren und Schwimmen. ${ }^{248}$

${ }^{244} \mathrm{Vgl}$. Hess, 1984, S. 174, Wössner, 1966, S. 5, Heiß, 1977.

${ }^{245} \mathrm{Vgl}$. Hess, 1984, S. 176.

${ }^{246} \mathrm{Vgl}$. ebd.

${ }^{247}$ Vgl. Frey, 1991, S. 97

${ }^{248} \mathrm{Vgl}$. ebd. S. $98 \mathrm{f}$. 
Während eine Quantifizierung der dem Sport zuzuschreibenden Einsparungen im Gesundheitswesen, insbesondere auf dem Sektor der Herz-Kreislauf-Krankheiten, kaum möglich ist, verhält es sich hinsichtlich der externen Kosten des Sports etwas anders. Sportverletzungen sind statistisch leichter zu erfassen und die daraus resultierenden Kosten in Form von Behandlungskosten, Produktionsausfallkosten sowie Lohnfortzahlungskosten eher zu beziffern.

\section{Tabelle 22: Kosten von Sportverletzungen}

\begin{tabular}{|c|c|c|c|c|}
\hline \multicolumn{2}{|c|}{$\begin{array}{l}\text { Jung, 1982. Folgekosten von Sportunfällen im } \\
\text { Jahr } 1980\end{array}$} & \multicolumn{3}{|c|}{$\begin{array}{l}\text { Brendel, 1982, Kosten der Sportunfälle im } \\
\text { Jahr } 1976\end{array}$} \\
\hline Kostenart & $\begin{array}{l}\text { Kosten in } \\
\text { MRD DM }\end{array}$ & \multirow{3}{*}{\multicolumn{2}{|c|}{$\begin{array}{l}\text { Heilbehandlung und Kran- } \\
\text { kengeld }\end{array}$}} & $\begin{array}{l}\text { Kosten in } \\
\text { MRD DM }\end{array}$ \\
\hline $\begin{array}{l}\text { ambulante Behandlung für } \\
\text { etwa } 950.000 \text { Sportverletzte }\end{array}$ & 0,23 & & & 0,574 \\
\hline $\begin{array}{l}\text { stationäre Behandlungskosten } \\
\text { für ca. } 141.000 \text { Sportverletzte }\end{array}$ & 0,37 & & & \\
\hline \multirow{2}{*}{$\begin{array}{l}\text { Produktionsausfallkosten für } \\
\text { die etwa } 6,8 \mathrm{MIO} \text { sportunfall- } \\
\text { bedingten Arbeitsausfalltage }\end{array}$} & \multirow[t]{2}{*}{1,33} & \multirow{2}{*}{$\begin{array}{l}\text { Produktions- } \\
\text { ausfall }\end{array}$} & minimal & 1,65 \\
\hline & & & maximal & 5,64 \\
\hline $\begin{array}{l}\text { Lohnfortzahlungskosten für } \\
\text { die etwa } 6,8 \text { MIO sportunfall- } \\
\text { bedingten Arbeitsausfalltage }\end{array}$ & 0,97 & \multicolumn{2}{|c|}{ Lohnfortzahlung } & 1,08 \\
\hline \multirow[t]{2}{*}{ Gesamtbetrag } & \multirow[t]{2}{*}{2,9} & \multirow[t]{2}{*}{ Gesamtbetrag } & minimal & 3,304 \\
\hline & & & maximal & 7,294 \\
\hline
\end{tabular}

Quelle: Jung, D. u. Ulmer, H.-V.: "Bewegungsmangel - Gefahr für die Volksgesundheit?", in: Deutsches Ärzteblatt, 80. Jahrgang, Heft 37, September 1983, S. 63/64. Die Angaben von Brendel wurden selbiger, o. g. Quelle entnommen.

Tabelle 22 zeigt die Folgekosten von Sportunfällen, wobei die Zahlen von Jung auf Veröffentlichungen über Sportunfälle, persönlichen Gesprächen bei verschiedenen Institutionen (Krankenkassen, Versicherungen, DSB etc.) basieren. Die Werte von Brendel hingegen beruhen auf einer Schätzung, stimmen aber bezüglich des unteren Eckwertes mit denen von Jung überein. Interessant erscheint hierbei, daß selbst der untere Eckwert mit etwa DM 3,3 MRD die Höhe der gesamten öffentlichen Ausgaben für Sportförderung und Sportstätten des Referenzjahres 1976 übersteigt.

Eine Untersuchung von Hauser und Gläser (1985) über Verletzungen im alpinen Skisport weist bspw. jährliche Kosten in Höhe von DM $265 \mathrm{MIO}$ aus, von denen $28 \mathrm{MIO}$ aus ambulanten, $60 \mathrm{MIO}$ aus stationären Behandlungskosten und $175 \mathrm{MIO} \mathrm{DM}$ aus Kosten durch Arbeitsausfall resultieren. ${ }^{249}$

Last not least soll im Zusammenhang mit möglichen Negativauswirkungen des Sports auf eine Problematik hingewiesen werden, die erst in den letzten Jahren zu einer sol-

${ }^{249} \mathrm{Vgl}$. Hauser und Gläser, 1985, S. $77 \mathrm{ff}$. 
chen wurde: die Verringenung der Ozonschicht. Die Gefährdung insbesondere sehr junger, aber auch älterer Menschen, die an Tagen mit erhöhten Ozonwerten Sport treiben, ist längst unbestritten.

Dennoch wird, vorrangig im organisierten Sport, derartigen Meldungen wenig Bedeutung beigemessen. Der Sportterminkalender ignoriert solche Gefährdungen geradezu, indem bspw. Fußballspiele nach wie vor zu Uhrzeiten stattfinden, zu denen an den entsprechenden Tagen von medizinischer Seite vor größeren körperlichen Belastungen gewarnt wird. Auf diese Weise wird von seiten des organisierten Freiluftsports ein zusätzlicher negativer externer Effekt allererst geschaffen .

Auf eine ausführliche Diskussion möglicher negativer gesundheitlicher Auswirkungen des Hochleistungssports wie auch des Dopingmißbrauchs soll an dieser Stelle verzichtet werden, handelt es sich dabei doch um Tendenzen, die, wenngleich sehr bedauernswert und gefährlich, doch glücklicherweise nur eine Minderheit der Sporttreibenden betreffen.

Als Fazit bleibt festzuhalten, daß positive Auswirkungen des Sports auf das HerzKreislauf-System in aller Regel von sportmedizinischer Seite anerkannt werden. Ob und inwieweit die darin enthaltenen externen Effekte die oben genannten Kosten übersteigen und damit per Saldo von einer Förderungswürdigkeit des Sports aus allokationstheoretischer Sicht gesprochen werden kann, ist zu vermuten und kann geschätzt werden, läßt sich allerdings nicht belegen. Geht man von der Existenz eines fühlbaren externen Effektes aus, bleiben aber dennoch zwei Fragen offen:

1) Der Status quo relativ undifferenzierter Behandlung verschiedener Sportarten kann unter Berücksichtigung ihrer unterschiedlicher Auswirkungen auf die Gesundheit nicht nachvollzogen werden, eine deutlich stärkere Differenzierung nach gesundheitlichen Aspekten ist angebracht.

2) Des weiteren kann die direkte Förderung lediglich des organisierten Sports sowie die starke Ausrichtung indirekter Förderung auf diese Sportangebotsform nicht nachvollzogen werden, sind doch vom Sporttreiben unter dem Dach alternativer Anbieter grundsätzlich keine anderen Auswirkungen auf die Gesundheit zu erwarten.

\subsection{Soziale Aspekte des Sports}

Analog zu den Gesundheitswirkungen gibt es auch über die gesellschaftspolitische Bedeutung des Sports zahlreiche Thesen. Der Siebte Sportbericht der Bundesregierung berichtet bspw., der Sport sei ein Mittel, welches Einstellungen und Fähigkeiten vermittle, die abstrakt nur unvollkommen lehrbar sind. Sport verschaffe wichtige Erfah- 
rungen, wenn Sporttreibende selbstgesetzte Regeln befolgen. Der einzelne könne sich im Sport moralisch und sozial bewähren und erfahre durch das Befolgen von Regeln rechtliches Denken sowie eine Einübung von Mechanismen zur kontrollierten Konfliktlösung. Weiter, der Sport vermittle Identifikation und Identität, führe zu einer sozialen Integration unterschiedlicher Gruppen und Schichten und stärke damit die Gesellschaft. 250

Bevor die Existenz derartiger Auswirkungen diskutiert werden soll, muß abermals hinterfragt werden, inwieweit es sich dabei um externe Effekte handelt oder aber um Auswirkungen, die zwar wünschenswert erscheinen mögen, deren Nutzen jedoch internalisiert sind. ${ }^{251}$ Eine pauschale, unwiderlegbare Antwort auf diese Frage zu geben ist nicht möglich. Sie hängt zum einen vom jeweiligen Effekt und zum anderen von der betrachteten Zielgruppe ab.

Mögliche Auswirkungen wie die Vermittlung von Identifikation und Identität sowie die soziale und moralische Bewährung erscheinen unserem Wertesystem zufolge zweifelsfrei begrüßenswert, sie stellen jedoch kaum internalisierungsbedürftige externe Effekte dar. Vielmehr handelt es sich dabei um Eigenschaften, die zuallererst das jeweilige Individuum selbst betreffen, mithin um internalisierte Nutzen für die Nachfrager der jeweiligen Sportgüter.

Gleiches gilt für die im Sport zweifelsfrei gegebene Möglichkeit, soziale Kontakte zu knüpfen. Auch hierbei handelt es sich um einen Nebennutzen des Sports, der allerdings interner Natur ist.

Eine Argumentationsweise, die aus der Identität eines Individuums oder der Möglichkeit des Knüpfens sozialer Kontakte externe Effekte ableiten möchte, ist nach Ansicht des Verfassers weit hergeholt und unterstreicht Bonus' These von der Allgegenwärtigkeit des Phänomens öffentlicher Güter. So ließe sich das Rad weiterdrehen und als logische Konsequenz könnten sämtliche Faktoren, die in irgendeiner Weise auf die Identität eines Individuums einwirken oder das Knüpfen sozialer Kontakte ermöglichen, als subventionierungswürdig deklariert werden.

Leichter nachvollziehbar ist das Vorhandensein externer Effekte im Zusammenhang mit der Erfahrung rechtlichen Denkens oder der Einübung von Mechanismen zur kontrollierten Konfliktlösung. Hier handelt es sich durchaus um Aspekte, aus denen auch Dritte einen Nutzen ziehen können. So sind mit Attributen wie rechtlichem Denken oder kontrollierter Konfliktlösung Eigenschaften angesprochen, die - im Gegensatz zu den Begriffen Identifikation und Identität eines bestimmten Menschen - explizit auf das Verhalten eines Individuums gegenüber seiner sozialen Umwelt fixiert sind.

${ }^{250} \mathrm{Vgl}$. Bundesministerium des Innern, 1991, S. $14 \mathrm{ff}$.

${ }^{251}$ Hierzu mehr im Rahmen der Betrachtung meritorischer Aspekte in Abschnitt 1.4. 
Dies wird bspw. deutlich, wenn man von einer latenten Kriminalität bei Jugendlichen sozialer Randgruppen ausgeht. Die Behauptung, der Sport verhindere mögliche Straftaten (und nutze damit auch Dritten), indem er den oben genannten Randgruppen eine sinnvolle Freizeitbeschäftigung bietet, erscheint unter diesem Aspekt in Einzelfällen insbesondere in Wohngegenden mit hohen Kriminalitätsraten zumindest nicht abwegig. Kaum zu vertreten ist hingegen der Schluß, aus dieser Randerscheinung oder irgendwelchen sonstigen Extrembeispielen ein pauschales, allokationstheoretisch begründetes Argument zugunsten öffentlicher Sportförderung abzuleiten. 252

Abgesehen von dieser Problematik der Definition potentieller Nutzen des Sporttreibens als Effekte externer oder interner Natur stellt sich schließlich die Frage, inwieweit die oben genannten Auswirkungen tatsächlich auftreten.

Auf seiten der Sportwissenschaft werden mögliche Auswirkungen des Sports diskutiert, wobei unter anderem die Gewinnung von Eigenschaften wie Konflikt-, Kooperations- und Kommunikationsfähigkeit sowie kommunikative und soziale Kompetenz angesprochen werden. ${ }^{253}$ Die Ansichten sind konträr.

Von medizinisch-psychologischer Seite wird grundsätzlich davon ausgegangen, daß der Sport die psychische Gesundheit des Menschen fördert, wenngleich einschränkend auch immer wieder darauf verwiesen wird, daß sich positive Wirkungen primär bei klinischen Gruppen zeigten. Gleichfalls wird der Mangel der Unsystematik empirischer Studien betont und auf die Notwendigkeit differenzierter Untersuchungspläne hingewiesen. So kommen etliche Untersuchungen zu dem Schluß, daß Sportler im Vergleich zu Nichtsportlern offener, kontaktfreudiger, ausgeglichener und weniger depressiv seien. Es bleibt jedoch ungewiß, ob diese Merkmale Auswirkungen des Sports sind, oder ob es sich hierbei um Voraussetzungen handelt, die die Aufnahme sportlicher Aktivitäten überhaupt erst fördern. 254

Auch die Sportsoziologie hat sich derartiger Fragestellungen angenommen. Becker (1989) hinterfragt die Tauglichkeit des Sports als sozialpolitisches Entsorgungs- und Steuerungsinstrument und konstatiert, daß man die Bedeutung des Sports insgesamt nicht überbewerten dürfe.25s Ähnlich kann wohl Klein (1989) interpretiert werden, wenn er von Möglichkeiten spricht, die im Sport als Mittel gegen soziale Probleme ge-

${ }^{252}$ Es sei daran erinnert, daß auch die Beseitigung von Ineffizienzen Kosten verursacht was letztlich als Argument gegen eine Internalisierung kleinerer Externalitäten angesehen werden kann. Der Autor vertritt die Ansicht, daß es sich bei diesem, wenngleich durchaus zu erwartenden externen Effekt um eine eher vernachlässigbare Größe handelt.

${ }^{253} \mathrm{Vgl}$. Becker, 1982, S. 10.

${ }^{254} \mathrm{Vgl}$. u.a. Andel/ Austin, 1984, Folkins/ Sime, 1981 oder Knobloch, 1979.

${ }^{255} \mathrm{Vgl}$. Becker, 1989, S. 188/89. Ferner wird unterstellt, daß es sich hierbei kaum um externe Effekte handelt. 
geben sind, gleichzeitig aber auch auf ungelöste soziale Probleme verweist, die durch den Sport produziert würden. 256

Einige empirische Untersuchungen im Rahmen der Aggressionsforschung kommen zu dem Schluß, daß im Sport erworbenes, aggressives Verhalten zu sozial erworbenem Normalverhalten wird und verweisen darauf, daß die Bereitschaft, physische Gewalt über das im Regelwerk verankerte $\mathrm{Maß}$ hinaus anzuwenden, mit steigender Alters- und Spielklasse steigt.257 Derartige, sportspezifische Sozialisationsprozesse aggressiver Verhaltensmuster im wettkampforientierten Sport sind allerdings nicht auf alle Sportarten zu verallgemeinern, sondern vorrangig in körperbetonten Mannschaftsball- und puckspielarten festzustellen.

Längst bekannt sind solche Negativeffekte aus dem Zuschauersport, dennoch gibt es auch hier keine einheitliche Meinung. Zwei Hypothesen beschäftigen die Wissenschaft:

Verfechter der sogenannten Katharsis-Hypothese vertreten die Ansicht, der Sport beinhalte eine sublimierende, reinigende Kraft, was z.B. dazu führen könne, daß durch Sport ein Aggressionsabbau erreicht werde. ${ }^{258}$ Derartige Wirkungen konnten allerdings bislang nicht nachgewiesen werden. Durch empirische Untersuchungen wurden eher gegensätzliche Tendenzen wie Steigerungen in der Aggressionsbereitschaft belegt. ${ }^{259}$ Allein die Polizeieinsätze bei Profifußballspielen in Baden-Württemberg, die vorrangig als Präventivmaßnahme gegen Ausschreitungen vor, während und nach den Spielen getroffen werden, kosteten das Land in der Saison 1991/92 DM 3,23 MIO. ${ }^{260}$ Rechnet man diese Kosten auf die knapp 40 deutschen Bundesligavereine hoch, dann dürften Polizeikosten in einer Größenordnung von mindestens DM 25 MIO jährlich durchaus realistisch sein. Weitere ausschreitungsbedingte Kosten wie Körperverletzungen, Zerstörungen von Stadionanlagen, Geschäftsauslagen, Vorgärten, städtischen Verkehrsmitteln und Bundesbahnzügen sind in dieser Rechnung nicht enthalten. ${ }^{261}$

Auch diesbezüglich muß einschränkend hinzugefügt werden, daß derart negative Auswirkungen keineswegs das ganze Sportphänomen als solches, sondern nur be-

\footnotetext{
${ }^{256} \mathrm{Vgl}$. Klein, 1989, S. 68.

${ }^{257}$ Vgl. u.a. Frogner/ Pilz, 1982, Smith, 1979, Vaz, 1979 oder Heinilä, 1974.

${ }^{258} \mathrm{Vgl}$. u.a. Lorenz, 1963 und Lang, 1976. Letzterer sieht allerdings auch die umgekehrte Möglichkeit, daß durch Massenveranstaltungen wie dem Zuschauersport Aggressionen allererst aufgebaut werden können.

${ }^{259} \mathrm{Vgl}$. Gutachten "Sport und Gewalt", bei Pilz u.a., 1982, S. 15.

${ }^{260} \mathrm{Vgl}$. Badische Zeitung vom 29.08.1992, "Klubs sollen Polizei bezahlen, Antrag der Grünen zum Polizeieinsatz in Fußballstadien" und vom 01.02.1993, "Klubs sollen künftig für Polizeieinsätze zahlen, 50 Millionen Einsparungen jährlich - 150 Polizisten beim SC-Spiel gegen Zürich."

${ }^{261} \mathrm{Vgl}$. hierzu Weis, 1982, S. $61 \mathrm{ff}$.
} 
stimmte Sportarten, primär körperbetonte, publikumsintensive Mannschaftssportarten betreffen; weniger publikumsintensive Disziplinen wie auch körperlose und Einzelsportarten bleiben davon eher verschont.

Es kann festgehalten werden: Die Nutzen einiger der eingangs genannten, möglichen Auswirkungen des Sports sind interner Natur und damit für die hier vorliegende Fragestellung einer allokationstheoretisch begründeten Subventionierungswürdigkeit nicht von Bedeutung.

In bezug auf jene, sehr wohl mit externen Effekten behafteten Auswirkungen des Sports auf das Sozialverhalten kann resümiert werden, daß sowohl externe Nutzen als auch Kosten vorliegen. Eine eindeutige Aussage hinsichtlich des sich einstellenden Nettoeffektes läßt sich nicht ableiten, weshalb öffentliche Sportförderung auch kaum mit dem Hinweis auf verhaltensorientierte externe Effekte gerechtfertigt werden kann.

\subsection{Sport als Standortfaktor}

Ein denkbarer externer Effekt des Sports, der sich keineswegs auf bestimmte Ausprägungen dieses Terminus beschränkt, sondern vielmehr zur allokationstheoretischen Erklärung öffentlicher Subventionierung sämtlicher Sportangebote beitragen kann, ist in dessen Funktion als Standortfaktor als zu sehen.

Standortfaktororientierte positive externe Effekte des Sports können darin bestehen, daß die gewerbliche Wirtschaft einer Kommune von der Existenz von Sportangeboten profitiert. Konkreter, Badeanstalten, Sportplätze und hallen gehören zur allgemeinen Infrastruktur. Dies wird beim Blick auf die große Anzahl sporttreibender Menschen ganz deutlich. Sportangebote können als eines mehrerer Kriterien bei der Einschätzung des Freizeitwertes verschiedener Kommunen durch Arbeitskräfte angesehen werden. Mit anderen Worten, eine sportausübende Arbeitskraft wird sich c.p. für jene Kommune entscheiden, in der sie das ihrer Einschätzung zufolge beste Sportangebot antrifft. ${ }^{262}$ Positive pekuniäre externe Effekte für die gewerbliche Wirtschaft resultieren folglich in Form sportangebotsinduzierter Steigerungen des Arbeitsangebots.

Führt ein Sportangebot darüber hinaus zu vergleichsweise stärkerer unternehmerischer Ansiedlung, dann kann als Folge dessen auch das zunächst relativ benachteiligte heimische Arbeitsangebot aus möglichen sportangebotsinduzierten Steigerungen der Arbeitsnachfrage profitieren. Eine derartige Argumentation erscheint aus den oben ge-

${ }^{262}$ Diese Bedeutung wird dann noch offensichtlicher, wenn man unterstellt, daß sich Arbeitsuchende ohne konkretes Stellenangebot für eine Kommune entscheiden und dann vor Ort auf Stellensuche gehen. 
nannten Gründen plausibel, wird sich doch das standortsuchende Unternehmen c.p. wiederum für jenen Standort entscheiden, an dem es das größte Sport- und damit auch Arbeitsangebot vorfindet.

Als Nettoeffekt kann ein Nutzengewinn für beide Seiten des Arbeitsmarktes resultieren. So könnte eine Erhöhung von Arbeitsnachfrage und -angebot in gleichbleibender Relation bspw. dazu beitragen, strukturelle Arbeitslosigkeit zu reduzieren. ${ }^{263}$

Nicht zuletzt muß die Kommune selbst bzw. der Wettbewerb zwischen den Kommunen in die Betrachtung einbezogen werden. Führt ein reichhaltiges Angebot tatsächlich zu einer Ansiedlung von Unternehmen, dann resultieren hieraus höhere Einnahmen insbesondere durch die zu erwartende Gewerbesteuer. Hiervon profitiert im Endeffekt wiederum die Einwohnerschaft als Ganzes.

Neben der Unmöglichkeit, das Ausmaß dieses gleichwohl vorhandenen externen Effektes zu quantifizieren, bleibt wiederum unklar, wieso sich die Sportförderung nicht auch auf den kommerziell angebotenen Sport erstreckt, sind die genannten Effekte doch von allen denkbaren Sportangebotsformen gleichermaßen zu erwarten.

\subsection{Sport als Werbeträger}

Auch, aber nicht nur mit der Konsequenz einer Ansiedlung von Unternehmen sind Werbeeffekte, insbesondere medienwirksamer Sportarten und -ereignisse ${ }^{264}$ im Bereich des Hochleistungssports denkbar.

Zwei Fragen sind zu klären: Existieren sportinduzierte Steigerungen des Bekanntheitsgrades einer Kommune oder Region und - falls ja - resultieren daraus externe Effekte bzw. worin können selbige im einzelnen bestehen?

Zunächst zur Frage des generellen Werbewertes des Sports. Thesen wie "die Bundesligazugehörigkeit eines örtlichen Fußballvereins macht die Stadt in der ganzen Nation, die Teilnahme einer Fußballbundesligamannschaft im Finale eines europäischen oder

\footnotetext{
${ }^{263}$ Anzufugen ist, daß sich obige Auswirkungen auf diejenige Gebietskörperschaft beschränken, innerhalb deren Einzugsbereich das jeweilige Sportangebot bereitgestellt wird. Aus gesamtstaatlicher Sicht handelt es sich um keinen Nutzengewinn, führen doch bspw. Zuzüge von Arbeitskräften zu einer Schlechterstellung der gewerblichen Wirtschaft in den Gebietskörperschaften, aus denen diese Arbeitskräfte abgewandert sind.

${ }^{264} \mathrm{Als}$ medienwirksam in der Bundesrepublik Deutschland ist zunächst der Profi-Fußballsport anzusehen, aber auch etliche weitere Sportarten wie Tennis-, Skisport etc. sind regelmäßig Objekte von Pressemeldungen
} 
sonstigen hochkarätigen Wettbewerbs gar in der ganzen Welt bekannt", gehören zum alltäglichen Sprachgebrauch der Sportförderungsbefürworter und Sportfunktionäre. ${ }^{265}$ Nun dürfte unbestritten sein, daß Erfolg im Sport aufgrund dieser Medienwirksamkeit tatsächlich gewisse Auswirkungen auf den Bekanntheitsgrad einer Kommune haben kann. Sportberichterstattungen nehmen in Fernsehen, Rundfunk und Printmedien einen großen Stellenwert ein. Sportlicher Erfolg, das Ausrichten von Spitzensportveranstaltungen o.ä. führt zu Pressemeldungen mit der Konsequenz, daß der Name einer Kommune oder eines Landes unter Umständen wiederholt genannt wird und die betreffende Körperschaft auf diesem Weg Bekanntheit erlangt. Kommunen wie Gelsenkirchen oder Kaiserslautern (Fußball), Gummersbach (Handball), Tauberbischofsheim (Fechten), um nur einige Beispiele zu nennen, haben zumindest unter Sportinteressierten einen Bekanntheitsgrad erreicht, den sie ohne den Sport wohl nicht hätten.

Zur Überprüfung dieser Hypothese wurden exemplarisch die Bekanntheitswirkungen des weiter oben genannten Freiburger Fußballbundesligisten untersucht.

Hierzu wurde in den Städten Berlin, Göttingen, Heilbronn, Magdeburg und Würzburg eine Umfrage mit knapp 1.000 Auskunftspersonen verschiedenen Alters und Geschlechts durchgeführt. ${ }^{266}$ Die gestellte Frage lautete "Woran denken Sie ganz spontan, wenn sie den Namen Freiburg im Breisgau hören?"

Tabelle 23 veranschaulicht sowohl die absolute Anzahl der Nennungen, die in den oben genannten Städten auf die jeweiligen Begriffe entfielen als auch deren prozentualen Anteil an den gesamten Nennungen innerhalb jeder Kommune. Es zeigte sich, daß die These vom Sport als Bekanntheitsfaktor durchaus vertreten werden kann.

$12,7 \%$ aller Nennungen bezogen sich auf den SC Freiburg. Damit war der SC Freiburg nach der Universität (19,4 \%) und dem Schwarzwald (17,3 \%) der am meisten erwähnte Begriff. ${ }^{267}$ Dies erscheint insbesondere deshalb bemerkenswert, als es sich hierbei um einen Verein handelt, der zum Zeitpunkt der Erhebung gerade einmal ein Jahr in der ersten Fußballbundesliga spielte.

\footnotetext{
${ }^{265}$ So bspw. argumentierte ein Vertreter des DFB im Zusammenhang mit der Diskussion über eine mögliche Beteiligung der Kommunen an den Vereinseinnahmen aus dem Verkauf der Fernsehübertragungsrechte während einer Sitzung des Sportausschusses des Deutschen Städtetages im April 1994, an welcher der Autor teilnehmen konnte.

${ }^{266}$ Die Auskunftspersonen (AP'n), zu etwa $40 \%$ Frauen, waren gleichermaßen auf die drei Altersgruppen $20-30,30-45$ und $>45$ verteilt. Maximal waren drei Nennungen pro Auskunftsperson möglich.

${ }^{267}$ Einige wenige Befragte, die den SC Freiburg nicht genannt hatten, bemerkten interessanterweise nach erwünschter Erläuterung des Befragungszwecks, daß sie sehr wohl an selbigen gedacht hätten, aus Gründen der Scham jedoch nach anderen Begrifflichkeiten suchten, erschien ihnen die Nennung eines Fußballvereins doch zu trivial.
} 
Tabelle 23: Werbeträger der Stadt Freiburg

\begin{tabular}{|l|l|l|l|l|l|l|l|l|l|l|l|l|l|}
\hline Städte: & \multicolumn{2}{|c|}{ Berlin } & \multicolumn{2}{|c|}{$\begin{array}{c}\text { Göttin- } \\
\text { gen }\end{array}$} & \multicolumn{2}{|c|}{$\begin{array}{c}\text { Heil- } \\
\text { bronn }\end{array}$} & \multicolumn{2}{c|}{$\begin{array}{c}\text { Magde- } \\
\text { burg }\end{array}$} & \multicolumn{2}{c|}{$\begin{array}{c}\text { Würz- } \\
\text { burg }\end{array}$} & \multicolumn{2}{c|}{ Gesamt } \\
\hline & abs. & in $\%$ & abs. & in $\%$ & abs. & in $\%$ & abs. & in \% & abs. & in \% & abs. & in $\%$ \\
\hline Anzahl der AP'n & 230 & & 143 & & 210 & & 220 & & 109 & & 912 & \\
\hline kein spont. Gedanke & 97 & & 53 & & 49 & & 129 & & 16 & & 344 & \\
\hline Anzahl Nennungen & 183 & & 149 & & 243 & & 124 & & 133 & & 832 & \\
\hline Nennungen pro AP'n & 0,8 & & 1,1 & & 1,2 & & 0,6 & & 1,2 & & 0,9 & \\
\hline Universität & 29 & 15,8 & 32 & 21,5 & 50 & 20,6 & 19 & 15,3 & 31 & 23,3 & 161 & 19,4 \\
\hline Schwarzwald & 30 & 16,4 & 22 & 14,8 & 45 & 18,5 & 27 & 21,8 & 20 & 15 & 144 & 17,3 \\
\hline SC Freiburg & 29 & 15,8 & 15 & 10,1 & 20 & 8,2 & 20 & 16,1 & 22 & 16,5 & 106 & 12,7 \\
\hline schöne Stadt/Fair & 19 & 10,4 & 18 & 12,1 & 29 & 11,9 & 7 & 5,6 & 14 & 10,5 & 87 & 10,5 \\
\hline Münster & 15 & 8,2 & 15 & 10,1 & 24 & 9,9 & 8 & 6,5 & 13 & 9,7 & 75 & 9 \\
\hline Wein & 11 & 6 & 8 & 5,4 & 3 & 1,2 & 20 & 16,1 & 5 & 3,8 & 47 & 5,6 \\
\hline Klima/Wetter & 9 & 4,9 & 10 & 6,7 & 12 & 4,9 & 2 & 1,6 & 2 & 1,5 & 35 & 4,2 \\
\hline Bächle & 5 & 2,7 & 4 & 2,7 & 15 & 6,2 & 1 & 0,8 & 7 & 5,3 & 32 & 3,8 \\
\hline Frankreich/Schweiz & 8 & 4,4 & 7 & 4,7 & 3 & 1,2 & 4 & 3,2 & 7 & 5,3 & 29 & 3,5 \\
\hline Ökostadt & 3 & 1,6 & 6 & 4,0 & 11 & 4,5 & & & 1 & 0,8 & 21 & 2,5 \\
\hline Urlaub & 7 & 3,8 & 4 & 2,7 & 2 & 0,8 & 6 & 4,8 & & & 19 & 2,3 \\
\hline Eishockey, EHC & & & & & 3 & 1,2 & & & 1 & 0,8 & 4 & 0,5 \\
\hline Verschiedenes & 18 & 9,8 & 8 & 5,3 & 26 & 10,7 & 10 & 8,1 & 10 & 7,5 & 72 & 8,7 \\
\hline
\end{tabular}

Tabelle 23 dokumentiert zudem, daß die relative Nennung des Begriffs SC Freiburg abgesehen von der auffallend hohen Quote im Erhebungsort Würzburg (16,5\%)268 mit zunehmender geographischer Entfernung des Befragungsortes von der Stadt Freiburg anstieg. Entfielen in Heilbronn nur 8,2 und in Göttingen 10,1\% der Nennungen auf den SC Freiburg, waren es bspw. in Berlin 15,8 \%, in Magdeburg gar 16,1 \%. Dies überrascht nicht, ist doch zu erwarten, daß jedwede Stadt hinsichtlich ihrer architektonischen und ähnlichen Sehenswürdigkeiten oder Charakteristika in weiter entfernten Regionen grundsätzlich einen niedrigeren Bekanntheitsgrad besitzt, der aus dem Fußball resultierende Bekanntheitsgrad hingegen durch die Sportberichterstattung in den überregionalen Medien gleichmäßiger verteilt ist.

\footnotetext{
${ }^{268}$ Dies könnte daher rühren, daß die Befragung in Würzburg eine Woche nach Beendigung der Bundesligasaison 1993/94 durchgefuihrt wurde und die Fußballgeschehnisse damit noch besonders aktuellen Charakter besaßen. Der SC Freiburg konnte in letzter Minute den Klassenerhalt sichern. In den anderen Städten wurde die Befragung drei bis vier Wochen später durchgeführt.
} 
Zur zweiten Frage: "Resultieren aus einer Steigerung des Bekanntheitsgrades externe Effekte?"

Neben einer Verstärkung oben diskutierter Standortvorteile sind im Zusammenhang mit medienwirksamen Sportgeschehnissen weitere externe Nutzen zu erwarten. Zunächst einmal kann der Besuch von Spitzensportveranstaltungen in der jeweils austragenden Ortschaft Nachfrage entfalten und damit Einkommen entstehen lassen. So liegt es auf der Hand, daß die auswärtigen Besucher von Sportveranstaltungen für die Zeit ihres Aufenthalts in der jeweiligen Stadt zumindest Leistungen der Gastronomiebranche nachfragen, unter Umständen kommen Nachfragesteigerungen im Einzelhandel, dem Hotelgewerbe und möglicherweise in weiteren Branchen hinzu.

Geht man von einem durchschnittlichen Besuch von 10.000 auswärtigen Gästen ${ }^{269}$ bei Bundesligaspielen aus und unterstellt als unteren Eckwert einen durchschnittlichen Konsum in Höhe von nur 10,- DM pro Kopf, dann ergibt dies pro Saison eine sportinduzierte Nachfrage in Höhe von 1,7 MIO DM. Es ist durchaus vorstellbar, daß der tatsächliche, von Ort zu Ort differierende Betrag diese 1,7 Millionen deutlich übersteigt. Verläßliche Zahlen zu diesem Punkt sind nicht bekannt, Schätzungen gehen jedoch zumeist von höheren Beträgen aus. So befürchten bspw. Nürnberger "Wirtschaftsexperten" als Folge des Abstiegs des 1. FC Nürnberg aus der ersten Fußballbundesliga Mindereinnahmen in Gastronomie und Einzelhandel in Höhe von 30 MIO DM pro Saison. 270

Daneben sind weitere, über den reinen Besuch von Sportereignissen hinaus entstehende Umsatzsteigerungen in der Tourismusbranche denkbar, die allerdings kaum geschätzt werden können. ${ }^{271}$

An diesem Punkt lassen sich die Überlegungen in gewisser Weise auch auf den Freizeit- und Breitensport übertragen. So wird die Wirtschaft, vorrangig die Tourismusbranche, insbesondere in jenen Regionen profitieren, die sich durch landschaftliche

\footnotetext{
${ }^{269}$ Diese Zahl scheint keineswegs zu hochgegriffen, müssen auswärtige Besucher doch nicht unbedingt Besucher aus der Stadt des Gastvereins sein. Der durchschnittliche Zuschauerzuspruch lag in der Saison 1992/93 bei etwa 25.000 Zuschauern pro Spiel. Vgl. Kicker, Spezial - 30 Jahre Bundesliga, S. 29.

${ }^{270} \mathrm{Vgl}$. Badische Zeitung vom 2.8.1994, "Wertvoller und besser als die teuerste Werbekampagne".

${ }^{271}$ Der Versuch, für die Städte Freiburg, Karlsruhe und Stuttgart konkrete Angaben über das finanzielle Ausmaß sonstiger überregionaler Werbemaßnahmen zu erhalten, schlug fehl. Lediglich von der Freiburger Wirtschafts- und Touristik-GmbH konnten die Kosten verschiedener Einzelmaßnahmen der Stadt Freiburg in Erfahrung gebracht werden. Die Freiburger Wirtschafts- und Touristik-GmbH wirbt bspw. auf Messen in aller Welt, wofur ein jährlicher Etatposten in Höhe von 90.000,- DM zur Verfuigung steht. Daneben wurde unlängst eine Anzeigenserie in der Welt am Sonntag geschaltet (drei Schaltungen zu je 1/8 Seite), wofür knapp 70.000,- DM ausgegeben wurden. Für weitere Anzeigen werden jährlich etwa 20.000,- DM verausgabt. Schließlich begleitet die FWT-GmbH den Freiburger Sportclub zu Auswärtsspielen mit ihrem Infomobil, um vor Ort Werbung für die Stadt zu betreiben.
} 
Gegebenheiten von anderen Regionen abheben und für das Angebot bestimmter Sportarten wie Ski- oder Wassersport geradezu prädestiniert sind.

Was für den Werbewert des Sports und daraus möglicherweise resultierender externer Effekte für Kommunen gilt, läßt sich auch auf den gesamten Staat übertragen.

Richtet eine bestimmte Nation eine Weltmeisterschaft in einer populären Sportart oder gar Olympische Spiele aus, dann werden sowohl Austragungsort bzw. -orte als auch der gesamte Staat für die Zeit dieses Wettkampfes zum Objekt etlicher Pressemeldungen. Der Sport kann folglich dazu dienen, einen Staat nach außen zu repräsentieren, sein Ansehen in der Welt positiv zu beeinflussen ${ }^{272}$ und damit möglicherweise Auswirkungen auf internationale Beziehungen und Außenhandel haben.

Ohne deren exaktes monetäres Ausmaß quantifizieren zu können soll festgehalten werden, daß das Angebot von Spitzensportgütern bzw. dessen Bekanntmachungseffekt einige externe Effekte impliziert.

Hieraus läßt sich eine allokationstheoretisch begründete kommunale Förderung von Sportstätten für den Zuschauersport, bspw. den Profifußball erklären. ${ }^{273}$ Für den Freizeit- und Breitensport gelten diese Überlegungen bspw. dann, wenn der Sport aufgrund besonderer geographischer Gegebenheiten dazu dienen kann, Touristen anzulocken.

\subsection{Sport - die wichtigste Nebensache der Welt}

Eine völlig andere Gruppe von Nutzen stellen die mit der "wichtigsten Nebensache der Welt" einhergehenden intangiblen Nutzen dar. ${ }^{274}$ Was fallt unter diesen Begriff? Sportliche Großereignisse, aber auch vergleichsweise bescheidene Wettkämpfe wie Lokalderbys u.ä. sorgen innerhalb von Teilen der sportinteressierten Bevölkerung für Gesprächsstoff und eine gewisse Identifikation mit den jeweiligen "Idolen" oder "Dream Teams". Weitere Nutzengewinne, insbesondere als Folge sportlicher Erfolge

\footnotetext{
272Insbesondere für Diktaturen war der Sport von jeher ein geeignetes Mittel zur nationalen Repräsentation. Man denke dabei an die Erfolge von Sportlern aus verschiedenen Ländern Osteuropas oder Hitlers Versuch, Deutschland durch die Ausrichtung der Olympischen Spiele in Berlin und GarmischPartenkirchen in ein positives Licht zu rücken.

${ }^{273}$ Diese Auffassung impliziert jedoch keineswegs ein bedingungsloses Festhalten an der gängigen Praxis der öffentlichen Bereitstellung von Zuschauersportarenen. Gleichermaßen denkbar wäre ein Modell, demzufolge private Investoren die jeweiligen Anlagen erstellen als auch unterhalten und an die betreffenden Gebietskörperschaften verpachten oder - möglicherweise veranstaltungsweise - direkt an die Sportanbieter vermieten. Im letztgenannten Fall wäre eine öffentliche Förderung durch Mietzuschüsse vorstellbar.

${ }^{274}$ Auch Gesundheitswirkungen können teilweise als intangible, d.h. völlig unbewertbare Kosten oder Nutzen bezeichnet werderı, vgl. Musgrave 1987, S. 202 f.
} 
bestehen in lokalen, regionalen oder gar nationalen Prestigeeffekten, woran nicht zuletzt auch Politikern gelegen sein dürfte. 275

Handelt es sich hierbei um externe Effekte oder sind derartige Nutzengewinne internalisiert? Zunächst einmal kann festgestellt werden: Resultierende Nutzen beschränken sich keineswegs auf die Besucher von Veranstaltungen, die für den Einlaß in aller Regel Eintrittsgebühren bezahlen. Auch über sonstige Informationskanäle können sich Interessierte in Kenntnis setzen. Es stellt sich die Frage, inwieweit Ausschluß von diesem, mit der Inkenntnissetzung verbundenen Nutzengewinn möglich ist.

Für den Erwerb von Printmedien oder für den Konsum von Leistungen der öffentlichen Rundfunk- und Fernsehanstalten sind Marktpreise zu entrichten. Ähnliches gilt für die Leistungen der privaten Anstalten, die sich über Werbung finanzieren, womit die Verbraucher letztlich über gestiegene Einkaufspreise für die Informationsgewinnung bezahlen.

Die Überlegungen zeigen, daß eine Internalisierung dieser Nutzen größtenteils gegeben ist. Ein völliger Ausschluß bei mangelnder Zahlungsbereitschaft ist jedoch keineswegs gewährleistet, da bspw. durch unrechtmäßige Nichtanmeldung von Rundfunk- und Fernsehgeräten die Möglichkeit besteht, sich über die jeweiligen Ereignisse sowohl des Spitzen- als auch des Breitensports zu informieren.

Keineswegs intangibler Natur sind hingegen jene Nutzen, die die Vermarkter von Zuschauersportware erzielen. ${ }^{276}$ Die oben genannten Medien profitieren in nicht unerheblichem Maße von der Sportberichterstattung, indem selbige zu höheren Einschaltquoten, höheren Auflagen und in aller Regel - daraus resultierend - zu höheren Werbeeinnahmen führen. Die Nutzen sind teilweise externer Natur, teilweise internalisiert. Zunächst zum Hochleistungssport: Die internationale Sportrechteverwertungsgesellschaft (ISPR) bezahlt für die Übertragungsrechte der Fußballbundesliga für den Zeitraum von 1992 bis 1997 insgesamt 650 Millionen DM.277 Weitaus weniger internalisiert sind die Nutzen, die den Printmedien aus der Berichterstattung entstehen. Diese profitieren überdies nicht nur vom Spitzen- sondern auch vom Breitensport, wie der Umfang der Sportberichterstattung vor allem in den Montagsausgaben etlicher Tageszeitungen belegt.

${ }^{275}$ Nutzengewinne in Form von Prestigeeffekten können freilich auch auf Breitensportebene, bspw. im Zusammenhang mit gemeindlichen Rivalitäten insbesondere bei Lokalderbys etc. bestehen.

276 Nutzen erlangt bspw. auch die Sportartikelindustrie, die im Sog steigender Attraktivität einer erfolgreichen Sportart mit höheren Verkaufszahlen rechnen kann. Vgl. Heinemann, 1988, S. 71.

${ }^{277} \mathrm{Vgl}$. "Wirtschaftsunternehmen Bundesliga. Die Gentlemen bitten zur Kasse", in Kicker Sportmagazin, Spezial, 30 Jahre Bundesliga, S. 28/29, Nümberg, 1993. 
Es kann festgehalten werden: Der Zuschauersport verursacht intangible, jedoch größtenteils internalisierte Effekte. Reale Effekte entstehen für die Vermarkter der jeweiligen Güter, die jedoch ebenfalls, insbesondere beim Fernsehen, größtenteils internalisiert sind.

\subsection{Sport und Ökologie}

Nicht erst seit den Olympischen Winterspielen 1992 im französischen Albertville, die als bis dahin größter Eingriff sportlicher Architektur in die Umwelt gelten, ist der Sport unter umweltpolitischen Gesichtspunkten in Verruf geraten. Negative Umweltwirkungen und damit externe Kosten des Sports stehen in der sportpolitischen Diskussion schon länger auf der Tagesordnung. ${ }^{278}$

Nachdem sich die Sportverbände mit diesem unliebsamen Thema zunächst sehr schwer taten, wurde nach und nach versucht, sich der Problematik anzunehmen. Der Deutsche Sportbund richtete 1982 eine Präsidialkommission "Sport und Umwelt" ein, ein Großteil der Fachverbände schuf Stellen für Umweltschutzbeauftragte, Symposien wurden veranstaltet, kurzum, der organisierte Sport ging in seinem Bemühen, sich selbst zu verteidigen, mehr und mehr in die Offensive. ${ }^{279}$

Gleichzeitig wurden - nicht zuletzt unter dem Druck der Öffentlichkeit und dem gestiegenen Umweltbewußtsein - sowohl von seiten der Politik als auch der Sportverbände potentielle ökologische Gefahren des Sports erkannt und in ihrer Bedeutung respektiert.

All dieser Bemühungen zum Trotz wurde und wird das Handeln des Deutschen Sportbundes auch weiterhin kritisiert. So weiß bspw. Digel zwar von gewissen Lemprozessen auf Seiten der Sportverbände wie unter anderem der Entscheidung des Deutschen Skiverbandes, sich für ein Verbot von Neuerschließungen von Skigebieten in der BRD einzusetzen, zu berichten. ${ }^{280}$ Gleichzeitig bemängelt er jedoch, die Umweltpolitik des DSB sei unglaubwürdig, wenn von Golffachverbänden Slogans wie "Golf - Der grüne Aufschwung" zu hören sind, wenn der Sport Verbindungen eher mit der Sportartikeloder der Automobilindustrie als mit den Umweltschützern selbst eingeht und wenn auf individuelle Verhaltensweisen abzielende Kampagnen den Konflikt Sport und Umwelt auf ein individuelles Problem reduzieren. ${ }^{281}$

${ }^{278}$ Eine umfangreiche Bibliographie zu dieser, seit Beginn der 80er Jahre aktuellen Problematik findet sich bei Klein, 1989

${ }^{279}$ Vgl. u.a. Erz, 1985, Deutscher Sportbund 1986, 1987.

${ }^{280} \mathrm{Vgl}$. Digel, 1989, S. 118.

${ }^{281}$ Womit er eine Aufklärungskampagne des o.g. Deutschen Skiverbandes zum richtigen Verhalten in der Natur anspricht. Vgl. Digel, 1989, S. $87 \mathrm{ff}$. 
Worin liegen nun im einzelnen die potentiellen externen Kosten des Sports in ökologischer Hinsicht?

Generell werden Landschaftszerstörung und -verbrauch durch Sportausübung und -architektur, das insbesondere mit dem Zuschauersport einhergehende hohe Verkehrsaufkommen und auch die mit Sportveranstaltungen verbundenen Lärmbelästigungen kritisiert oder gerichtlich angegangen. Eine nach Kosten und auch Sportarten differenzierende Betrachtung ist angebracht.

Sportstätten erfordern zuweilen große Flächen, was in dicht besiedelten Städten erhebliche Probleme mit sich bringt. Bauland ist knapp und der Ausweis von Flächen als Sportzentren sicherlich nicht im Interesse eines Antisportlers, der unter den teuren Grundstückspreisen leidet.

Entstehen Sportstätten hingegen außerhalb, auf der grünen Wiese, so führen sie zu unter Umständen unerwünschten Veränderungen des Landschaftsbildes.

Daneben geht mit dem Phänomen Sport, insbesondere sportlichen Großereignissen, immer auch die Problematik eines ohnehin zu großen Verkehrsaufkommens mit all seinen bekannten Nachteilen einher.

Werden Sportstätten zentral erstellt, dann führen sie in der Regel zu einer zusätzlichen Belastung der bereits viel zu vollen Ștraßen. Eine Ansiedlung am Stadtrand oder auBerhalb der Stadt hingegen erhöht das Verkehrsaufkommen insofern, als dadurch die Anfahrtswege vergrößert werden. Diesbezüglich stellt sich allerdings die Frage, inwieweit hier von einem sportspezifischen externen Effekt gesprochen werden kann, oder aber inwieweit eine fehlerhafte Verkehrspolitik Ursache verkehrsbedingter Umweltbelastungen ist. ${ }^{282}$

Ein Aspekt, der insbesondere bezüglich des Streits zwischen Befürwortern und Gegnern des Sports auf gerichtlicher Ebene Bedeutung erlangt hat, sind schließlich die mit dem Sport einhergehenden Lärmbelästigungen. Hiermit werden primär die im Zusammenhang mit Freiluftsportarten auftretenden Lärmemissionen bei abendlichen Trainingseinheiten oder auch dem Spielbetrieb am Wochenende angesprochen.

Neben diesen allgemeinen, auf eine Vielzahl von Sportarten zutreffenden Charakteristika, konzentriert sich die umweltpolitisch orientierte Kritik am Sport auf einige einzelne Sportarten. Die unter dem Aspekt der Landschaftszerstörung am meisten beanstandeten Sportarten sind der Ski-, der Wasser- und der Motorsport. So wird insbeson-

282Durch das Verkehrsaufkommen im Zusammenhang mit sportlichen Großereignissen entstehende Umweltbelastungen können durch eine weitsichtige Verkehrspolitik eingedämmt werden. Hierzu zählt u.a. eine durch preisliche Anreizmechanismen initiierte Verlagerung des Individual- auf den öffentlichen Personennahverkehr. Von diesen Möglichkeiten wird derzeit noch kein allzugroßer Gebrauch gemacht. 
dere erstgenannter Sportart vorgeworfen, sie trage zur Zerstörung der natürlichen Vegetationsdecke, zur Artenveränderung der Vegetation, zur Begünstigung von Hangrutsch, Bodenerosionen, Lawinen und ähnlichem bei.

Auch der Golfsport wird von umweltpolitischer Seite nicht zuletzt seines immensen Landschaftsverbrauchs wegen stark kritisiert. Ähnlich verhält es sich mit dem Eissport, dessen gewaltiger Energieaufwand zur künstlichen Eisgewinnung bemängelt wird.

Es soll festgehalten werden: Der Sport kann durchaus negative externe Effekte beinhalten, die von Sportart zu Sportart differieren. Die Beantwortung der Frage, ab welchem Punkt von einem internalisierungsbedürftigen externen Effekt gesprochen werden kann, ist allerdings nicht unproblematisch. So kann es nicht Sinn und Zweck sein, jegliche Sportausübung unter dem Aspekt einer Vermeidung von Lärm, Verkehr und ähnlichem einzustellen.

Die Bewertung und Entscheidung obliegt letztlich den sportpolitischen Entscheidungsträgern und auch der Justiz, die bereits in verschiedenen höchstrichterlichen Urteilen die Nutzung von Sportstätten, insbesondere aus Gründen der damit einhergehenden Lärmbelästigungen eingeschränkt hat. ${ }^{283}$

\footnotetext{
${ }^{283}$ Im Tennisplatz-Urteil des BGH vom 17.12.1982 wurde der Klage der Eigentümerin eines benachbarten Grundstücks auf zeitliche Beschränkung des Spielbetriebes aus Lärmgründen stattgegeben. Ähnliches im Tegelsbach-Fall, in dem die Anwohnerin einer Sportanlage von der Stadt Hamburg verlangte, den durch Freizeitsport verursachten Lärm zu mindern. Dieser Klage wurde vom OVG stattgegeben, das BVerwG hat dieses Urteil weitgehend bestätigt. Vgl. Hagen, 1992, S. I ff.
} 


\subsubsection{Sportgüter und Rivalität im Konsum}

Die theoretischen Ausführungen haben gezeigt, daß das Fehlen jeglicher Rivalitäten im Konsum eine öffentliche, über Steuern finanzierte Bereitstellung erklären kann. Anders hingegen im Falle begrenzter Vorteile kollektiver Nutzung und einer Nachfrage nur bestimmter Teile der Grundgesamtheit. Das Prinzip der fiskalischen Äquivalenz legt eine Finanzierung des Gutes durch die jeweiligen Nutzer, die begrenzten Vorteile verschiedenartige preispolitische Praktiken nahe. Welcher Natur sind nun Sportgüter?

\subsubsection{Aktive Sportausübung}

Im Gegensatz zur Untersuchung der externen Effekte gestaltet sich die Betrachtung dieses zweiten Kriteriums für das Vorliegen eines öffentlichen Gutes relativ problemlos.

Sportgüter zeichnen sich größtenteils durch begrenzte Vorteile kollektiver Nutzung, d.h. $0<\tilde{n}<1$, also einem Auftreten von Rivalitäten bzw. Überfüllungskosten erst ab dem Erreichen bestimmter, je nach Sportart oder Sportstätte verschiedener Kapazitätsgrenzen aus. 284

Starke Vorteile kollektiver Nutzung gewähren bspw. Badeanstalten, Skipisten, Langlaufloipen oder Eisstadien. Auf derartigen Sportanlagen kann die Anzahl der Nutzer pro Zeiteinheit ${ }^{285}$ ohne sofortiges Auftreten fühlbarer Grenzüberfüllungskosten deutlich erhöht werden.

Ebenfalls Vorteile kollektiver Nutzung, wenngleich in geringerem Maße, bieten die primär privat bereitgestellten Fitneß-, Tanz- und Aerobicstudios sowie Rasen- oder Tenneflächen, Leichtathletikbahnen und Sporthallen. Auf diesen letztgenannten, zur

\footnotetext{
${ }^{284}$ Daneben kann auch der Nutzungszweck, die Unterscheidung zwischen Training und Wettkampf, als Indikator für das Erreichen von Kapazitätsgrenzen herangezogen werden. Bedingt durch die Regelwerke verschiedener Sportarten ist die Kapazitätsgrenze unter Wettkampfgesichtspunkten in einigen Sportarten exakt festgelegt. Das Fußballspiel bspw. ist auf 22 Teilnehmer normiert, ähnliches gilt für andere Sportarten. Bei einer über diese Grenze hinausgehenden Nachfrage müssen Verdrängungskosten in Kauf genommen werden. In den weiteren Ausführungen sollen derartige, durch das sportartenspezifische Reglement begründeten, vollständig fixen Kapazitätsgrenzen vernachlässigt werden. Es erfolgt eine Betrachtung nicht wettkampforientierter Nutzungen von Sportstätten.

${ }^{285}$ Die Möglichkeit der Nutzung von Sportstätten zu alternativen Zeitpunkten ist nicht relevant. Wenngleich dies unter Umständen kaum Kosten in Form zusätzlicher Bereitstellungskosten (von einer möglichen anteiligen Verrechnung von Abschreibungen wird abstrahiert) verursachen mag, für den Benutzer bedeutet ein Ausweichen aufgrund von Überfüllung das Hinnehmen von Verdrängungskosten und kann somit als Rivalität im Konsum aufgefaßt werden. Folglich wird in den weiteren Ausführungen jeweils die möglicherweise auftretende Rivalität pro Nutzungsakt betrachtet.
} 
Ausübung verschiedener Sportarten geeigneten Sportanlagen, variiert die Schwelle des Eintretens von Überfüllungskosten mit der jeweils ausgeübten Sportart.

Wird eine Rasensportstätte bspw. von einer Gymnastikgruppe belegt, dann läßt sich die Anzahl der Teilnehmer deutlich erhöhen, ohne zugleich fühlbare Überfüllungskosten zu erzeugen. Wird die Anlage hingegen von einer Fußballmannschaft zu Trainingszwecken genutzt, dann kann die Anzahl der Aktiven zwar auch gesteigert werden, Überfüllung wird allerdings schneller eintreten als im Gymnastikbeispiel.

Ein besonderes Charakteristikum von Mannschaftssportarten ist darin zu sehen, daß gar eine gewisse Mindestanzahl von Teilnehmern erforderlich ist, um überhaupt eine den Spezifika der jeweiligen Sportart entsprechende Sportausübung zu ermöglichen.

Bezogen auf die Bereitstellungsbedingung im Buchanan-Modell bedeutet dies, daß bis zu einem gewissen Punkt mit dem Hinzukommen weiterer Teilnehmer negative Grenzkosten in Form resultierender Kostenreduzierung aus der vergrößerten Mitgliederzahl nicht mit ebenfalls negativen, sondern positiven Grenznutzen einhergehen. Dieser Punkt läßt sich als die sportartenspezifische, den jeweiligen Regeln entsprechende Teilnehmerzahl interpretieren. So wird die Erhöhung der Teilnehmeranzahl im Beispiel Fußball bis zum 22. Spieler positive Grenznutzen erzeugen, erst ab einer weiteren Ausdehnung der Teilnehmerzahl werden die Grenznutzen negativ, fühlbare Überfüllung setzt ein.

Wird oben genannter Rasenplatz schließlich von einem Hammer- oder Diskuswerfer genutzt, dann zeigen sich starke Rivalitäten im Konsum.

Grundsätzlich schwache Vorteile kollektiver Nutzung bieten demgegenüber Tennisund Badmintonfelder oder auch Squashcourts. Es führt zu einer starken Verfremdung des Tennissports, wenn sechs anstelle der normalerweise zwei oder vier Spieler gleichzeitig auf einem Feld aktiv sind. Noch deutlicher wird dies in Squashcourts, wo eine Ausweitung der Teilnehmerzahl gar zur Gefahr für die körperliche Unversehrtheit der Aktiven wird.

Die Betrachtung der verschiedenen Sportgüter zeigte, daß das eingangs formulierte, vorweggenommene Ergebnis bestätigt werden kann. Sportgüter sind Klubgüter, wenngleich festgehalten werden muß, daß das Ausmaß der Rivalitäten zwischen verschiedenen Sportarten und -stätten stark differiert.

Nun ergaben die theoretischen Ausführungen, daß für die Bereitstellung derartiger, im Konsum nicht vollständig rivalisierender Güter ein Angebot durch einen Klub, ${ }^{286}$ also

${ }^{286}$ Der Begriff des Klubs im Sinne der gleichnamigen Theorie ist keineswegs mit dem identischen, oftmals als Synonym für den Begriff Sportverein verwandten Terminus gleichzusetzen. Ein Klub muß kein Sportverein sein. Darüber hinaus sei abermals darauf hingewiesen, daß nicht in der marktmäßigen Bereitstellung als solcher eine Ineffizienz begründet liegt, sondern in der marktwirtschaftlichen Unter- 
eine Gebührenerhebung in Form lediglich eines mengenabhängigen Preises in Höhe der Grenzkosten oder eines two-part-tariffs mit möglicherweise verschiedenartiger Aufteilung auf die beiden Preisbestandteile effizient ist.

Ein Blick auf die preispolitische Praxis von Sportanbietern verdeutlicht folgendes: Der organisierte Sport erhebt grundsätzlich mengenunabhängige Preise in Form von Mitgliedschaftsgebühren. Diese Mitgliedschaftsgebühren sind im allgemeinen einziger Bestandteil der Gebührenerhebung und berechtigen ohne weitere Zahlungen zur Nutzung der jeweiligen Anlage.

Die weiter oben diskutierten, zusätzlichen mengenabhängigen Tolls, sei es in Höhe der Grenzkosten oder auch davon abweichend, sind in der preispolitischen Praxis des Vereinssports kaum anzutreffen. Überdurchschnittlich hoher Nachfrage zu Stoßzeiten wird in aller Regel nicht durch einen Ausschluß über Preismechanismen wie Rationierungsgebühren oder ähnlichem begegnet.

$\mathrm{Da}$ Überfüllung aber dennoch nur bis zu einem gewissen Grad akzeptiert wird, sorgen andere Mechanismen für eine zeitliche Verteilung der Nutzung. In einigen Sportarten erfolgt dies durch eine Vergabe fixer Benutzungszeiten für bestimmte Personengruppen, zumeist getrennt nach Alter und/ oder Spielstärke. In anderen Sportarten findet der Ausschluß nach dem Prinzip "Wer zuerst kommt, mahlt zuerst" statt. So bspw. in Tennisvereinen, wo sich die Mitglieder ihre Spielzeiten durch rechtzeitiges Eintragen in Listen reservieren.

Überdurchschnittlich hohen Beitrittsgesuchen wird hingegen sehr wohl mittels preislicher Ausschlußmechanismen begegnet. So nehmen insbesondere Aufnahme- aber auch Mitgliedschaftsgebühren in Sportarten wie Tennis oder Golf zuweilen Größenordnungen an, die für einen Teil der Nachfrager prohibitiven Charakter haben. Gängig ist darüber hinaus eine persönliche Differenzierung der Mitgliedsbeiträge primär in Abhängigkeit vom Alter der Mitglieder.

Es kann festgehalten werden: Die preispolitische Praxis des organisierten Sports entspricht den theoretischen Ausführungen insoweit, als grundsätzlich eine mengenunabhängige Preisbildung anzutreffen ist. Ineffizienzen können jedoch darin bestehen, daß potentielle Wenignutzer oder einmalige Nutzer aufgrund der für sie unter Umständen prohibitiven Mitgliedschaftsgebühren aus dem Markt gedrängt werden. Es stellt sich die Frage, inwieweit solchermaßen Ineffizienzen insbesondere dort, wo Kapazitäten zeitweise unausgelastet sind, nicht durch ein Nebeneinander von mengenunabhängigen und -abhängigen Preisen eingedämmt werden könnten.

Geläufig ist eine derartige Preispolitik bspw. im Zusammenhang mit kommunalen Badeanstalten, wo zum einen Jahreskarten angeboten werden, die dann unbegrenzt freien

nehmungen in aller Regel unterstellten Gebührenerhebung in Form mengenabhängiger Preise in Höhe der Durchschnittskosten. 
Eintritt gewähren. Zum anderen werden Einzeltickets verkauft, was für Wenignutzer den Vorteil hat, nicht durch prohibitive Saisontickets aus dem Markt gedrängt zu werden.

$\mathrm{Da}$ mengenabhängige Preise auch im organisierten Sport nicht gänzlich unbekannt sind, belegt ein Blick auf die Praxis einiger Tennisvereine. Dort wird oftmals Gästen, allerdings nur gemeinsam mit einem Mitglied, gegen Bezahlung mengenabhängiger Gebühren das Recht zur Benutzung eingeräumt. Grossekettler bezeichnet dies im Falle nicht überlasteter Vereinsgüter als Ineffizienz, die notwendig ist, um das Abwandern von Mitgliedern zu verhindern. ${ }^{287}$ Der Autor vertritt die Ansicht, daß eine Überlassung an Nichtmitglieder gegen die Bezahlung mengenabhängiger Gebühren auch als Möglichkeit angesehen werden kann, die bestehenden Ineffizienzen aufgrund der Verdrängung von Wenignutzern aus dem Markt zu reduzieren. So wäre doch eine grundsätzliche Öffnung von Vereinsanlagen für Nichtmitglieder insbesondere zu Zeiten denkbar, in denen die Vereinsanlagen erfahrungsgemäß ohnehin nicht ausgelastet sind. Die Ineffizienzen einer Erhebung mengenabhängiger Gebühren für solche Nutzergruppierungen sind sicherlich geringer als jene leerstehender Anlagen.

Zum kommerziell angebotenen Sport: Entgegen der in der Literatur vielfach verbreiteten Annahme, gewinnmaximierende Unternehmen erheben grundsätzlich Durchschnittskostenpreise, zeigen sich auch in der preispolitischen Praxis kommerzieller Sportanbieter Formen mengenunabhängiger Gebühren, bspw. Monatsbeiträge in den im Konsum weniger stark rivalisierenden Fitneßzentren, Tanzstudios und dergleichen. Auch privatwirtschaftliche Unternehmungen können sich folglich wie Klubs verhalten. In den im Konsum weitaus stärker rivalisierenden Squash-, Badminton- und Tennisanlagen ist hingegen eine stundenweise Vermietung von Plätzen mit mengenabhängigen Preisen üblich.

Ferner bedienen sich auch kommerzielle Anbieter der Preisdifferenzierung. Im Gegensatz zum organisierten Sport wird hierbei jedoch primär nicht nach persönlichen, sondern nach zeitlichen Gesichtspunkten differenziert. Persönliche Preisdifferenzierung erfolgt bei einigen Anbietern zusätzlich. ${ }^{288}$

Vergleicht man die mit der Charakterisierung als Klubgüter einhergehenden, theoretisch begründeten Anforderungen an die Form der Entgeltpolitik solcher Güter mit den preispolitischen Praktiken von Vereinen und kommerziellen Anbietern, dann läßt sich eine pauschale Aussage für oder wider eine dieser beiden Angebotsformen nicht tref-

\footnotetext{
${ }^{287} \mathrm{Vgl}$. Grossekettler, 1985, S. 240

288Preisdifferenzierung kann dazu führen, den Auslastungsgrad der jeweiligen Anlage zu erhöhen, wenngleich kommerzielle Anbieter dies kaum zur Reduzierung allokativer Ineffizienzen, sondem eher zur Erhöhung des Gewinns anstreben dürften.
} 
fen. Eine Bereitstellung durch einen Sportverein scheint allerdings immer dann effizienter, wenn

a) gegebene Vorteile kollektiver Nutzung und vorhandene Unteilbarkeiten Angebotsgrößenordnungen nahelegen, die zu quasimonopolistischen Marktstrukturen führen, eine privatwirtschaftliche, gewinnmaximierende Bereitstellung somit $\mathrm{zu}$ erzwungenen Mengenverknappungen bzw. Preiserhöhungen führt. Von derartigen Marktstrukturen kann im Zusammenhang mit Sportgütern insbesondere in kleineren Kommunen ausgegangen werden; das weiter oben beschriebene Berglas-Modell hat damit für Sportmärkte kaum praktische Bedeutung. 289

b) unabhängig obiger Marktstrukturen erwerbswirtschaftliche Durchschnittspreis-Kalkulationen wie im Bereich von Tennis- oder Squash-angeboten unter Umständen zu unausgelasteten Kapazitäten führen. 290

Die gleichwohl vorhandenen, weiter oben dargestellten Ineffizienzen der preispolitischen Praxis von Sportvereinen verweisen auf eine Second-Best-Lösung und erklären darüber hinaus die Präsenz privatwirtschaftlicher Unternehmen als Ergänzung zum Vereinsangebot, insbesondere um Gelegenheitsnutzer nicht vollständig aus dem Markt zu drängen. Kommerzielle Angebote können damit auch als Auffangbecken für Ineffizienzen vereinsmäßiger Bereitstellung verstanden werden.

So wird ein homo oeconomicus c.p. grundsätzlich jenes Angebot bevorzugen, welches unter finanziellen Aspekten das günstigste ist. Für Wenignutzer bietet sich eine Sportausübung unter dem Dach privater Anbieter an, Vielnutzer hingegen werden den mit Wettbewerbsvorteilen versehenen, öffentlich subventionierten Verein aufsuchen.

Völlig unabhängig von verschiedenen preispolitischen Gepflogenheiten lassen sich hingegen kommerzielle Fitneßzentren sowie Tanz- und Aerobicstudios erklären, da in diesem Bereich auch gewinnmaximierende Unternehmen mengenunabhängige Mitgliedschaftsgebühren erheben und sich damit wie Klubs verhalten. Private Anbieter dienen hierbei nicht als Auffangbecken für aus dem Markt gedrängte Wenignutzer, die Präsenz kommerzieller Anbieter liegt vielmehr darin begründet, daß vergleichbare Güter vom organisierten Sport nicht angeboten werden.

\footnotetext{
${ }^{289}$ Ausnahmen könnten tendenziell in Großstädten oder dicht besiedelten Regionen auftreten, wenn zumindest annäherungsweise von einem Wettbewerb zwischen den einzelnen Anbietern homogener Güter ausgegangen werden kann.

${ }^{290}$ Unausgelastete Kapazitäten auf Vereinsanlagen erscheinen zwar ebenfalls nicht wünschenswert, beruhen jedoch abgesehen von der kritisierten Nichtöffnung für Dritte grundsätzlich nicht auf ineffizienten Ausschlußmechanismen, sondern auf alternativen Konsumentscheidungen der Mitglieder.
} 
Weitere, nicht ausschließlich allokationstheoretisch bedingte subjektive Entscheidungskriterien der Sportanbieterwahl liegen in strukturellen Aspekten dieser beiden Organisationsformen begründet.

So bietet der Verein im Gegensatz zum kommerziellen Anbieter neben der Möglichkeit zur Sportausübung auch ein gewisses "Vereinsleben" an. Individuen, die diese Eigenschaft des Vereins schätzen, werden sich aus diesem Grund eher zum organisierten Sport hingezogen fuihlen. Andere hingegen lehnen exakt dieses, von ihnen als "Vereinsmeierei" betrachtete Charakteristikum des organisierten Sports ab und bevorzugen demgemäß private Angebote, welche ebenfalls eine soziale Funktion - wenngleich in geringerem Maße - erfüllen.

Ein weiterer Aspekt, der kommerzielle Anbieter für etliche Individuen interessant macht, liegt in der im Vergleich zum Verein nicht gegebenen Beschränkung der Sportpartner auf Mitglieder. Kommerzielle Anbieter gewähren den Sporttreibenden weitaus mehr Raum, den jeweiligen Spielpartner frei auszuwählen.

Ein gleichermaßen persönlich bedingtes Motiv zur Sportausübung unter dem Dach privatwirtschaftlicher Anbieter beruht auf terminlichen Aspekten. Sportvereine zeichnen sich zumeist durch fest vorgegebene Trainings- und auch Spielzeiten aus, ${ }^{291}$ was einer variablen zeitlichen Gestaltung der Sportausübung kaum Freiraum läßt. Für Individuen, die ihre Sportausübung aufgrund beruflicher oder sonstiger Umstände flexibel gestalten wollen oder müssen, schließt sich damit eine Sportausübung im Verein möglicherweise aus.

Persönlich bedingt ist schließlich eine grundsätzliche Abneigung gegenüber dem Wettkampfsport, welche einige Menschen dazu bewegt, sich vom Vereinssport zu distanzieren. Dessen starke Wettkampforientierung bietet Individuen, die eine rein freizeitsportliche Ausübung präferieren, kaum Möglichkeiten. ${ }^{292}$

Nachdem nunmehr die typischen "Kommerzsportnachfrager" skizziert sind, stellt sich darüber hinaus die Frage, wieso es gerade die Sportarten Tennis, Squash, Badminton, Kegeln, Schießen oder Reiten sowie Fitneß- und Tanzzentren sind, für die sich parallel zum organisierten Sport ein privater Markt entwickelt hat. Das Argument möglicherweise prohibitiver Mitgliedschaftsgebühren trifft doch auf die gesamte Angebotspalette des organisierten Sports zu.

Zum einen handelt es sich in sämtlichen Fällen um Individualsportarten, zum anderen erfolgt die Sportausübung unter dem Dach privater Anbieter in der Regel nicht wett-

\footnotetext{
${ }^{291}$ Dieses Argument triff bspw. nicht auf den Tennissport zu, da die Platzvergabe in Vereinen oftmals tage- oder wochenweise durch Eintragung in Listen erfolgt.

292Dies wird insbesondere dann deutlich, wenn Trainingseinheiten größtenteils aus Konditionsübungen, dem Einüben bestimmter Bewegungsabläufe oder - insbesondere bei Ballsportarten - dem Einstudieren bestimmter Schläge und Spielsituationen bestehen und für das reine Spiel letztlich wenig Zeit übrig bleibt.
} 
kampforientiert. Schließlich zeichnen sich die genannten Sportarten durch eine gewisse, zur Ausübung unverzichtbare Sportstätteninfrastruktur aus. Nicht zwingend konstitutiv scheint hingegen ein geringer Grad der Vorteile kollektiver Nutzung zu sein, wie die vergleichsweise weniger stark rivalisierenden Fitneß- und Aerobicstudios belegen.

Mehrere Aspekte erläutern, wieso es Sportstätten für den Individual- und eben nicht den Mannschaftssport sind, die von privater Seite angeboten werden. Mannschaftssportarten erfordern ex definitione das Zusammenkommen mehrerer Akteure, d.h. im Vergleich zu Individualsportarten höhere Transaktionskosten hinsichtlich der Planung von Zusammensetzung und Terminierung.

Berücksichtigt man, daß diese Planung bei kommerziellen Anbietern Aufgabe der jeweiligen Nutzer ist, ${ }^{293}$ dann zeigt sich folgendes: Nachfrager, die sich zeitlich nicht fixieren können oder wechselnde Sportpartner bevorzugen, stehen diesem Transaktionskostenaufwand wiederholt gegenüber. Da die Höhe dieser Transaktionskosten eine Funktion der Teilnehmerzahl darstellt, bietet sich zur Minimierung derselben die Ausübung von Sportarten mit geringer Teilnehmerzahl und damit eine Individualsportart an. Eine aus Sicht der kommerziellen Sportanbieter ausreichende bzw. gewinnermöglichende Nachfrage nach Sportstätten des Mannschaftssports ist kaum zu erwarten.

Ein unterstellter Zusammenhang zwischen grundsätzlicher Ablehnung des Vereinslebens und der Präferenz von Individualsportarten tendiert schließlich in dieselbe Richtung.

Zum Aspekt der freizeitsportlichen Orientierung des kommerziellen Sports: Die Existenz eines Wettkampfbetriebes mit verschiedenen Ligen und regelmäßigen Punktespielen erfordert eine gewisse Kooperation zwischen verschiedenen Anbietern und eine Organisation unter dem Dach einer übergeordneten Vereinigung. Eine derartige Kooperation ist Domäne des organisierten Sports und wird, wenngleich keineswegs zwingend, vom kommerziellen Sport nicht angeboten. Ausnahmen bilden die von privaten Anbietern gelegentlich organisierten Jedermann-Turniere in Sportarten wie bspw. Tennis, Squash oder Badminton. Etliche Squash-Anbieter haben darüber hinaus hausinterne Ranglisten geschaffen, wodurch ein gewisser Wettkampfcharakter der Sportausübung ennöglicht, aber nicht zwingend auferlegt wird. Derlei Angebote haben die Funktion, auch Nachfragern, die aus anderen Gründen als der Ablehnung von Wettkämpfen zur Klientel kommerzieller Anbieter gehören, eine gewisse Wettbe-

${ }^{293}$ Die denkbare Übertragung dieser Funktion auf den Anbieter käme wiederum dem Vereinsmodell sehr nahe. 
werbsatmosphäre zu gewähren, beschränken sich aber wiederum auf Individualsportarten. 294

Ein letzter Aspekt der Betrachtung potentieller Märkte für privatwirtschaftliche Unternehmen liegt schließlich in der mit verschiedenen Sportarten verbundenen sportstättenspezifischen Infrastruktur begründet.

Für private Angebote dürten überall dort keine Erfolgsaussichten bestehen, wo es sich um Kernsportarten handelt, die keiner speziellen Sportstätteninfrastruktur bedürfen. So "organisieren" sich Nichtvereinssportler oftmals in Freizeitsportgruppen und weichen sofern es die jeweilige Sportart erlaubt - zur Ausübung auf frei zugängliche Bolzplätze oder die grüne Wiese aus. ${ }^{295}$ Gleiches gilt, wenn diesen Freizeitsportgruppen kommunale Sporthallen zur Verfügung gestellt werden.

Zusammenfassend kann festgehalten werden: Privatwirtschaftliche Angebote können sich überall dort entwickeln, wo preispolitische oder sonstige strukturelle Charakteristika des organisierten Sports potentielle Nachfrager ausschließen, oder es sich um neuere Erscheinungsformen des Sports handelt, für die (noch) gar keine Angebote seitens des organisierten Sports bestehen. Der kommerzielle Sport wendet sich damit grundsätzlich an eine Klientel, der die Strukturen des organisierten Sports aus verschiedenen Gründen nicht gerecht werden und kann damit als Auffangbecken für Ineffizienzen und auch Marktlücken des organisierten Sports bezeichnet werden.

Eine Ausweitung kommerzieller Angebote auf Mannschaftsportarten, Wettkampfbetriebe und Sportarten, die keiner bestimmten Sportstätteninfrastruktur bedürfen, ist aufgrund mangelnder Ertragsaussichten nicht zu erwarten.

\subsubsection{Der Zuschauersport}

Was bezüglich der begrenzten Vorteile im Konsum für die aktive Sportausübung gilt, gilt für den Zuschauersport in noch stärkerem Maße. Wenn in eine mit 20.000 Zuschauern besetzte, aber 80.000 Zuschauer fassende Fußballarena 10.000 oder auch 20.000 Zuschauer hinzukommen, wird dies für die bereits Anwesenden keinerlei Grenzüberfüllungskosten bedeuten. Im Gegenteil: aufgrund besonderer Charakteristika

${ }^{294}$ Ein privatwirtschaftliches Angebot wettkampforientierten Mannschaftssports scheitert unabhängig von der auf diesem Sektor vorliegenden Dominanz des organisierten Sports an den weiter oben genannten Charakteristika sowohl des Mannschaftssports als auch des typischen "Kommerzsportnachfragers". Wettkampforientiert betriebener Mannschaftssport erfordert Kontinuität hinsichtlich Training und Ausübung, ein Team muB "aufeinander eingespielt" sein.

${ }^{295}$ So bspw. im Zusammenhang mit Fahrrad- und Lauftreffs, Gymnastikgruppen oder auch in Ballsportarten wie Fußball, Basketball, Hockey etc. für die unter Inkaufnahme einiger Abweichungen vom offiziellen Regelwerk keine besondere sportartenspezifische Infrastruktur vonnöten ist. 
des Gutes Zuschauersport wie bspw. Atmosphäre wird ein Großteil der Zuschauer, unabhängig jeglicher Durchschnittskostengedanken, einen stärkeren Zuschauerzuspruch eher begrüßen.

Zuschauersport zeichnet sich nun, obschon von Vereinen angeboten, keineswegs durch ausschließlich mengenunabhängige Preise aus. Wenngleich Dauerkarten angeboten werden und es einige wenige Vereine gibt, die einen Großteil ihres Kartenkontingents auch in Form von Dauerkarten verkaufen, ${ }^{296}$ ist die Entgeltpolitik gleichermaßen durch eine Erhebung von Eintrittspreisen pro Spiel gekennzeichnet.

Der Grund hierfür ist weniger in bestimmten Charakteristika der Anbieter von $\mathrm{Zu}$ schauersport zu sehen, ${ }^{297}$ sondern eher nachfragebedingt zu erklären. Der Anteil der Gelegenheitsbesucher an den Gesamtzuschauerzahlen ist nicht zu unterschätzen, ein ausschließlicher Verkauf der Eintrittskarten in Form saisonweiser Dauerkarten hätte folglich für etliche Nachfrager prohibitiven Charakter, wie er weiter oben als mögliche Folge von relativ hohen Mitgliedschaftsgebühren dargestellt wurde..$^{298}$

\subsubsection{Interpretation der Ergebnisse: Neoklassische Überlegungen als Erklä- rungsansatz für den Status quo von Sportförderung und die Angebots- struktur von Sportgütern?}

Inwieweit können die dargestellten neoklassischen Gedanken den Status quo öffentlicher Sportförderung erklären? Ein jeglicher Kritik standhaltendes Resümee zu ziehen, ist aufgrund der kaum lösbaren Probleme einer Bewertung möglicher externer Effekte nicht möglich.

Der Autor vertritt die Ansicht, daß die Frage der neoklassisch begründeten Förderungswürdigkeit von Gütern zur aktiven Sportausübung wesentlich von der Einschätzung externer Gesundheitseffekte, auftretender Standortvorteile sowie von der Bewertung externer Umweltkosten abhängt.

Durchaus denkbare positive Auswirkungen hinsichtlich der Entwicklung der Identität von Individuen sind primär internalisierte Effekte und können folglich kaum eine öffentliche Subventionierung erklären.

\footnotetext{
${ }^{296}$ Hierbei handelt es sich in erster Linie um Vereine mit besonders großem Zuschauerzuspruch. Der Grund für den Erwerb von Dauerkarten ist hierbei auch in der Unsicherheit des Erhalts einzelner Karten zu sehen.

${ }^{297}$ Jeder Bundesligist würde seine Eintrittskarten wohl liebend gerne zu einem Großteil in Form von Dauerkarten verkaufen.

${ }^{298}$ Es sei bspw. im Zusammenhang mit Fußballbundesligaspielen nur an die jeweiligen Fans der Gäste gedacht.
} 
Bezüglich der Auswirkungen auf das Sozialverhalten und damit tatsächlich möglicher externer Effekte scheint fraglich, inwieweit diese internalisierungsbedürftig sind. Trifft die These $z u$, daß es sich hierbei lediglich um Randerscheinungen handelt, so müssen, wie bereits weiter oben erwähnt, auch die mit öffentlichen Interventionen verbundenen Ineffizienzen berücksichtigt werden, die ein staatliches Eingreifen erst ab einer merklichen Größenordnung vorhandener externer Effekte nahelegen. Überdies zeigten die Ausfuihrungen, daß als Folge von Leistungsorientierung und Erfolgsdruck bspw. in körperbetonten Mannschaftsball- und -pucksportarten keineswegs von einem positiven Nettoeffekt ausgegangen werden kann.

Auch die mit der Charakterisierung des Sports als wichtigster Nebensache der Welt einhergehenden Nutzengewinne sind größtenteils internalisiert.

Unterstellt man neben den Standortvorteilen per Saldo positive externe Gesundheitseffekte und betrachtet man die Umweltbelastungen als nicht internalisierungsbedürftig, dann können die neoklassischen Gedanken einiges begreiflich machen und eine grundsätzliche Gewährung öffentlicher Gelder für den Sport erklären. Sie reichen jedoch keineswegs aus, die sportpolitische Subventionierungspraxis vollständig zu erklären. Einige Argumente mögen diese Aussage begründen.

Wenn der Sport aus Gründen positiver externer Effekte hinsichtlich der Gesundheit und des Standortaspektes fördenungswürdig erscheint, dann stellt sich die Frage, wieso nicht auch kommerziell angebotener Sport und weitere Sportangebotsformen im gleichen Maße öffentlich gefördert werden. Deren standortpolitische Bedeutung und Auswirkungen auf die Gesundheit dürten kaum anderer Natur sein.

Ebenfalls kann nicht erklärt werden, wieso unter gesundheitlichen Aspekten nicht eine stärkere Differenzierung zwischen verschiedenen Sportarten vorgenommen wird. Die Sportmedizin hat gezeigt, daß aerobe Sportarten gegenüber Schnellkraftsportarten vorzuziehen sind.

Bezüglich der Unterhaltung von Badeanstalten ist anzumerken, daß dem Schwimmen von sportmedizinischer Seite durchaus positive Gesundheitswirkungen zugestanden werden. Berücksichtigt man allerdings, da B Badeanstalten für etliche Besucher weniger Mittel zum Sport als zur Erholung darstellen, dann muß dieses Argument stark relativiert werden. Erholungsmaßnahmen mögen zwar durchaus gesundheitsfördernde Auswirkungen beinhalten, diese dürfen dann allerdings nicht als Auswirkungen des Sports angesehen werden. Überdies stellt sich im Zusammenhang mit den Badeantsalten angesichts deren Kostenintensität die Frage nach dem Verhältnis von Input zu Output 
Geht man des weiteren davon aus, daß der primäre Zweck öffentlicher Sportförderung in der Realisierung der "pareto-optimalen Sportnachfrage" liegt, dann stellt sich nach Ansicht des Autors die Frage, inwieweit hier nicht über das Ziel hinausgeschossen wird. Sind die öffentliche Bereitstellung von Sportanlagen oder auch die Gewährung von Investitionszuschüssen zur Erstellung privater Sportstätten im Sinne einer Anschubfinanzierung nicht unbedingt in ihrer absoluten Höhe, so aber doch vom Grundsatz her nachzuvollziehen, bedarf es zumindest zum Verständnis der vielfältigen laufenden Zuschüsse an die Sportvereine weiterer Erklärungsansätze. Ohne gezwungenermaßen eine finanzielle Schlechterstellung des Vereinssports zu implizieren, könnte bspw. durch eine Beschränkung der Sportförderung auf eine Anschubfinanzierung viel Verwaltungsaufwand eingespart werden. ${ }^{299}$

Hinsichtlich der Förderung des Zuschauersports, insbesondere des hier betrachteten medienwirksamen Profifußballs vertritt der Autor die Ansicht, daß die zu erwartenden externen Effekte in Form von Nachfragesteigerungen in den oben genannten Branchen eine öffentliche Förderung unter allokationstheoretischen Gesichtspunkten erklären können. Aussagen bezüglich der optimalen Höhe öffentlicher Subventionierung sind aufgrund von Bewertungsproblemen allerdings ebenso wenig möglich wie im Bereich der Sportausübung.

Die Theorie der Klubs konnte darüber hinaus einige Fragen zur Struktur von Sportangeboten, wie bspw. die Funktion kommerzieller Anbieter als Auffangbecken aus dem Markt gedrängter Konsumenten in den von beiden Anbietern angebotenen Sportarten klären.

Auf unterschiedliche Preispolitiken allein läßt sich die Frage nach dem Status quo der Angebotsstruktur jedoch keineswegs reduzieren. So zeichnen sich auch verschiedene kommerzielle Angebote durch eine mengenunabhängige Gebührenerhebung aus und schließlich handelt es sich dabei um Güter, die von Vereinen entweder nicht oder aber in anderer Form angeboten werden. Kommerzielle Angebote wurden grundsätzlich als Auffangbecken für Nachfragergruppierungen interpretiert, deren persönlicher Charakteristika die Struktur des organisierten Sports nicht gerecht wird.

Die weiteren theoretischen Erklärungsansätze versuchen jenen Teil des Erklärungsbedarfs zu erhellen, der mit den neoklassischen Gedanken allein nicht hinreichend beantwortet werden konnte.

${ }^{299}$ Selbst wenn man am bestehenden Förderungsvolumen festhalten wollte, wäre es bspw. möglich, den Vereinen anstelle etlicher kleiner Zuschüsse höhere Investitionszuschüsse zu gewähren. 


\subsection{Sport und Meritorik}

\subsubsection{Grundlagen der Meritorik}

Neben den oben dargestellten neoklassischen Modellen umfaßt die Theorie der öffentlichen Güter einen weiteren Erklärungsansatz öffentlicher Tätigkeit, die von Richard A. Musgrave begründete Theorie der meritorischen Güter. ${ }^{300}$

Öfentliche Tätigkeit beruht dieser Theorie zufolge nicht notwendigerweise auf mangelnder Möglichkeit zum Ausschluß oder nicht vorhandenen Rivalitäten im Konsum. Vielmehr werden staatliche Eingriffe in das Marktgeschehen damit erklärt, daß der Staat die Entscheidungssouveränität der privaten Konsumenten nicht akzeptiert. Eine Begründung, die mit einer individualistischen Staatsauffassung nur schwerlich zu vereinbaren ist. ${ }^{301}$

Was steckt hinter dem Begriff meritorisches Gut? Eine Begriffsdefinition als solche existiert nicht, da auch keine Kriterien existieren, die darüber entscheiden können, was das "richtige" Ausmaß an Bedürfnisbefriedigung ist. Meritorisch, so ließe sich vielleicht behelfen, ist ein Gut dann, wenn der Staat eine ungenügende Nachfrage seitens der Individuen unterstellt, demeritorisch hingegen, wenn die Nachfrage nach Ansicht der "better-informed-group" zu hoch ist. ${ }^{302}$ Es wird sehr schnell die politische Dimension offensichtlich, die hinter dieser "Definition?" steckt. Da ex definitione die je-

\footnotetext{
${ }^{300}$ Musgrave führte bereits 1957 den Begriff der merit wants ein. Vgl. Musgrave, 1957, S. 333 f. In der Folgezeit hat Musgrave diesen Begriff zunehmend konkretisiert. Vgl. Musgrave, 1957a, S. 111 und 1959, S. 13 f.

${ }^{301}$ Bezüglich der Vereinbarung meritorischer Güter mit den Konsumentenpräferenzen und damit einer individualistischen Lehre gibt es unterschiedliche Auffassungen. Werden die meritorischen Güter von Musgrave selbst aufgrund eines Eingriffs in die Konsumentenpräferenzen zunächst als nicht vereinbar mit dem individualistischen Ansatz bezeichnet, wird diese Auffassung in späteren Veröffentlichungen zunehmend gelockert. In der "Theory of Public Finance" erfolgt eine Unterscheidung zwischen merit wants und social wants. Letztere seien mit den Konsumentenpräferenzen durchaus vereinbar. Vgl. Musgrave, 1959, S. 14. 1984 hinterfragt Musgrave in der 4. Auflage von "The Public Finance in Theory an Practice" schließlich, ob es sich bei freiwillig gewährten Transfers ohne Annahmezwang überhaupt um eine Verletzung der Präferenzen handelt. Vgl. Musgrave, 1984, S. 79. Nichtsdestotrotz verweist er auf die im Zusammenhang mit social wants (Gemeinschaftsbedürfnissen) einhergehenden Gefahren diktatorischen Mißbrauchs.

Timm bspw. versucht, meritorische Staatstätigkeit mit einer individualistischen Auffassung dadurch zu vereinbaren, indem er auf Wahlen im politischen Entscheidungsprozeß hinweist, in denen die Individuen auch über die Bereitstellung von meritorischen Gütern abstimmen. Vgl. Timm, 1981, S. 155/156.

Deutschsprachige finanzwissenschaftliche Lehrbücher sehen in meritorischen Gütern zumeist einen Eingriff in die Konsumentenpräferenzen und damit auch Probleme einer Vereinbarung mit dem individualistischen Modell. Vgl. u.a. Andel, 1990, S. 388 f., Brümmerhoff, 1990, S. 96 f., Petersen, 1993, S. $144 \mathrm{f}$.

${ }^{302}$ Als demeritorisch werden in aller Regel Güter wie Alkohol oder Zigaretten betrachtet, deren Konsum durch Strafsteuern zu drosseln versucht wird.
} 
weiligen politischen Entscheidungsträger für die nicht unbedingt nachvollziehbare Beurteilung zuständig sind, entsteht ein Spielraum, der Platz läßt für jegliche Interpretationen, Mißbrauch nicht ausgeschlossen.

In der Literatur finden sich zumeist drei verschiedene, keineswegs unumstrittene Ansätze, meritorische Aspekte zu kanalisieren. Es sind dies der Interpretation von Head ${ }^{303}$ zufolge verzerrte Präferenzen der Individuen, distributive Überlegungen und zuletzt auch externe Effekte, auf die an dieser Stelle allerdings nicht mehr näher eingegangen wird. ${ }^{304}$

Die folgenden Ausführungen versuchen nachzuvollziehen, inwieweit Sportgüter meritorische Güter in dem Sinne sind, als sie aufgrund verzerrter Präferenzen oder zu geringer Einkommen nicht "ausreichend" nachgefragt werden.

Zuvor soll jedoch abermals festgehalten werden: Die "Definition" des Begriffs Meritorik impliziert, all das als meritorisch anzusehen, was die neoklassischen Überlegungen nicht erklären können. Die Theorie der meritorischen Güter könnte somit als Residuum aufgefaßt, das Buch geschlossen werden. Hiermit gibt sich der Autor nicht zufrieden.

\subsubsection{Sport und verzerrte Präferenzen}

Dieser Erklärungsansatz, von einer Rechtfertigung kann keine Rede sein, sagt aus, daß die Konsumenten aufgrund verzerrter Präferenzen falsche Konsumentscheidungen treffen, welche in der Folge durch den Staat korrigiert werden. Verzerrte Präferenzen wiederum basieren auf falscher oder unzureichender Information, konkret, der Unterschätzung der Nutzen bestimmter Güter, ${ }^{305}$ der Unterschätzung der Nutzung zukünftiger

\footnotetext{
${ }^{303} \mathrm{Vgl}$. Head, 1966, S. 3 ff. Andel hingegen sieht die konstitutive Eigenschaft meritorischer Güter primär im Aspekt der verzerrten Präferenzen begründet. Die Hinzunahme der externen Effekte sowie des Verteilungsaspekts durch Head schätzt er als Fehlinterpretation ein. Vgl. Andel, 1984, S. 640.

${ }^{304} \mathrm{Vgl}$. hierzu die Ausführungen weiter oben. Musgrave bringt die meritorischen Bedürfnisse zunächst nur mit Gütern in Verbindung, die dem Ausschlußprinzip unterliegen. In der 1959 veröffentlichten "Theory of Public Finance" wird der Begriff des meritorischen allerdings auch mit öffentlichen Gütern (externen Effekten) in Verbindung gebracht. So argumentiert Musgrave u.a., unentgeltliche Erziehung und kostenloser Gesundheitsdienst käme nicht nur den betroffenen Schülern und Patienten zugute. Vielmehr ziehe jedermann einen Nutzen daraus, wenn er in einer gebildeteren und gesünderen Gesellschaft leben könne. Vgl. Musgrave, 1959, S. 13.

Andel hingegen sieht in der Einschätzung Heads, gemäß welcher der Aspekt des öffentlichen Guts für Musgrave relevant sei, eine Fehlinterpretation. Vgl. Andel, 1984, S. 640.

Dies erscheint insofern plausibel, als man zur Erklärung einer staatlichen Intervention aufgrund des Vorliegens externer Effekte auf die mit der individualistischen Staatsauffassung wesentlich weniger konkurrierenden neoklassischen Theorien zurückgreifen kann.

${ }^{305}$ Als Beispiele werden Kulturgüter wie Opern und Museen genannt.
} 
Erträge ${ }^{306}$ sowie unzureichender Information infolge irrefuihrender Werbung oder hoher Informationskosten.

Kritisiert wird dieser Ansatz insbesondere unter Verweis auf die Frage, ob denn die staatlichen Entscheidungsträger vollkommene Informationen besitzen ${ }^{307}$ und falls ja, ob es nicht vorteilhafter wäre, die Ursachen der Fehlinformationen zu ergründen und die richtigen Informationen bereitzustellen. ${ }^{308}$ Dies wäre zum einen mit dem individualistischen Ansatz leichter in Einklang zu bringen und könnte darüber hinaus die Gefahr eines Mißbrauchs verhindern.

Inwieweit kann nun argumentiert werden, die Nachfrage nach Sportgütern sei infolge verzerrter Präferenzen zu gering?

Die erste Frage darf nicht lauten, "ist die private Nachfrage nach Sportgütern zu gering?" Sie muß vielmehr lauten, "erscheint die private Nachfrage den politischen Entscheidungsträgern zu gering?" Eine objektive Antwort kann somit nicht erfolgen, da der Autor kein politischer Entscheidungsträger ist.

Dennoch soll zu klären versucht werden, worin die Gründe dafür liegen könnten, öffentliche Sportförderung zu meritorisieren. $\mathrm{Zu}$ diesem $\mathrm{Zweck}$ ist es angebracht, auf jene Auswirkungen des Konsums von Sportgütern einzugehen, die innerhalb der Untersuchungen bezüglich der externen Effekte als möglicherweise positiv, jedoch zumindest teilweise interner Natur charakterisiert wurden. Angesprochen sind mögliche Auswirkungen auf die Gesundheit sowie verschiedene soziale Aspekte des Sports.

Zunächst zu den Gesundheitswirkungen: Trifft die These zu, daß der Sport der Gesundheit des Individuums dient, dann resultiert neben dem politisch geschaffenen externen Effekt in Form der gesetzlichen Krankenversicherung ein zukünftiger interner Nutzen heutigen Sporttreibens, den der jeweilige Konsument möglicherweise als zu gering bewertet. Diese denkbare Unterschätzung zukünftiger Nutzen kann nun als zu geringe Nachfrage aufgrund unvollkommener Information angesehen werden.

Offen bleibt freilich weiterhin die Frage, wieso die zukünftigen Gesundheitseffekte als Resultat heutigen Sporttreibens unter dem Dach kommerzieller Anbieter von den politischen Entscheidungsträgern nicht meritorisiert werden. Es ist demzufolge nicht das Sporttreiben als solches ein meritorisches Gut, sondern lediglich das Sporttreiben in Sportvereinen. Nach Ansicht des Autors kann damit auch eine auf zukünftigen Ge-

\footnotetext{
${ }^{306}$ Als Beispiele gelten in aller Regel die zu geringe Einschätzung zukünftiger Nutzen der Altersvorsorge oder der Ausbildung.

${ }^{307} \mathrm{Vgl}$. Andel, 1990, S. 389.

${ }^{308} \mathrm{Vgl}$. Mc Lure, 1968, S. 481. Andel nennt als mögliche Maßnahmen im Zusammenhang mit demeritorischen Gütern Kennzeichnungspflichten für die Produzenten oder auch die Unterbindung irreführender Werbung. Vgl. Andel, 1990, S. 390. Im Zusammenhang mit meritorischen Gütern könnte der Staat folglich Aufklärungskampagnen betreiben.
} 
sundheitswirkungen basierende Kategorisierung in meritorische und nichtmeritorische Güter nicht hinreichen, die öffentliche Subventionienungspraxis verständlich zu machen.

Etwas leichter nachzuvollziehen ist die Meritorisierung des Vereinssports, wenn man mögliche soziale Funktionen der beiden analysierten Sportanbieter betrachtet. Wenngleich soziale Aspekte weiter oben als primär internalisierte Nutzen angesehen wurden und darüber hinaus beiden Anbietern eine gewisse soziale Funktion zugesprochen wurde, läßt sich im Gegensatz zu den oben genannten Gesundheitswirkungen ein grundsätzlicher Unterschied zwischen Vereins- und kommerziellem Sport konstatieren. Berücksichtigt man bspw., daß der organisierte Sport auch Mannschaftssportarten, der kommerzielle Sport indes nur Individualsportarten anbietet, dann offenbart sich die bessere Eignung insbesondere des Vereins- respektive des Mannschaftssports, soziale Kontakte zu knüpfen und ganz allgemein, sich sozial zu bewähren. Zusätzliches Gewicht erfahren derartige Überlegungen schließlich unter Berücksichtigung weiterer, von Vereinen angebotener Aktivitäten abseits des reinen Sporttreibens.

\subsubsection{Verteilungspolitische Aspekte öffentlicher Sportförderung}

Ein weiterer Erklärungsansatz meritorisch motivierter Eingriffe besteht in der Annahme, bestimmte Konsumentengruppen würden einige Güter wie bspw. ärztliche Vorsorge oder Ausbildung aufgrund zu geringer Einkommen nicht nachfragen können.

Auch dem Vereinssport wird eine derartige, distributive Funktion zugebilligt. So argumentiert der Deutsche Sportbund, daß eine Übernahme der Funktionen des Sports durch kommerzielle Anbieter niemals zu einer solchen sozialen Offensive wie in den Vereinen führen würde, daß die sozial Schwächeren ausgesperrt und gegenüber den Stärkeren auf der Strecke blieben. ${ }^{309}$

Diese Argumentation scheint zunächst einleuchtend, vergleicht man die vom einzelnen aufzubringende finanzielle Last fuir die staatlich subventionierte Mitgliedschaft in einem Sportverein mit den Preisen in kommerziellen Sportzentren oder die Eintrittspreise kommunaler Badeanstalten mit denen privater Träger. Es liegt auf der Hand, daß sich Jugendliche oder ganz allgemein, sozial Schwächere eine Sportausübung möglicherweise nicht leisten könnten.

Andererseits wird dieser Ansatz deswegen kritisiert, weil es unbestreitbar bessere Instrumente zur Redistribution von Einkommen gibt. ${ }^{310}$ Die öffentliche Bereitstellung

${ }^{309} \mathrm{Vgl}$. Deutscher Sportbund, 1990, S. 36.

${ }^{310}$ Musgrave selbst schränkt die Bedeutung des umverteilungsorientierten Ansatzes ein, wenn er hinterfragt, ob es unter redistributiven Gesichtspunkten nicht vorzuziehen sei, Geldmittel zu verteilen und den Empfängern die Verwendung zu überlassen. Vgl. Musgrave, 1987, S. 102. 
meritorischer Güter schafft in aller Regel Mitnahmeeffekte, die jegliche redistributive Zielsetzungen unterwandert. So profitiert schließlich nicht nur der sozial Schwächere von den subventionierten Eintritts- oder Mitgliedschaftsgebühren. ${ }^{311}$

Wollte man effizient redistributive Zielsetzungen verfolgen wäre es sinnvoller, den tatsächlich Bedürttigen die eingesparten Mittel bisheriger öffentlicher Sportsubventionen in Form direkter Geldzuwendungen zukommen zu lassen. Mitnahmeeffekte würden unterbunden und für die sozial Schwächeren würde sich nichts ändern, im Gegenteil, sie wären unter Umständen per Saldo sogar die Gewinner. ${ }^{312}$ Sollten selbige das gestiegene verfügbare Einkommen zu anderen Zwecken als dem Sporttreiben verwenden, so beruhte dies auf ihrer Entscheidung, die sie als mündige Bürger zu treffen in der Lage sein sollten. Der Status quo hingegen stellt eine Verletzung der Konsumentenpräferenzen dar.

Ein solches Modell scheint unter verteilungspolitischen Gesichtspunkten wünschenswert, es stellt sich jedoch die Frage nach der politischen Durchsetzbarkeit. Solange eine Umverteilung direkt verfügbarer Einkommen, die parallel zu erfolgen hätte, nicht zu erwarten ist, stellt die derzeitige Second-Best-Lösung tatsächlich die einzige Möglichkeit dar, das Gut Sport auch den sozial Schwachen näherzubringen, womit auch die Argumentation der Sportverbände plausibel wird.

Die Diskussion hat gezeigt, daß sich der Sport neben gewissen externen Effekten auch durch verschiedene meritorische Komponenten auszeichnet. ${ }^{313}$ Der Autor gibt sich mit dieser Begriffsbezeichnung allerdings nicht zufrieden. Die Analyse der zu untersuchenden staatlichen Tätigkeit, eine Tätigkeit, über die letztlich von Politikern entschieden wird, bliebe unvollständig, würden nicht auch etwaige Eigeninteressen dieser politischen Entscheidungsträger in die Betrachtung miteinbezogen werden, womit die ökonomische Theorie der Politik angesprochen ist.

\footnotetext{
${ }^{311}$ Sogar eine Umverteilung nach oben ist denkbar, wenn man bspw. davon ausgeht, daß etwa Kulturgüter primär durch höhere Einkommensschichten nachgefragt werden.

${ }^{312}$ Dieser Fall schiene denkbar, wenn man die nunmehr von den sozial Stärkeren eingenommenen Gelder an die sozial Schwächeren umverteilen würde.

${ }^{313}$ Auch Andel charakterisiert den Sport als meritorisches Gut. Vgl. Andel, 1990, S. 388. 


\section{Polit-ökonomische Ansätze}

\subsection{Die Downs'sche Stimmenmaximierungshypothese}

Die von Schumpeter ${ }^{314}$ skizzierte und von Downs ${ }^{315}$ im einzelnen entwickelte Hypothese eines Stimmenmaximierungsverhalten der Politiker unterstellt eine aus Eigeninteressen der Politiker heraus initiierte Gewährung öffentlicher Mittel.

Es wird darin angenommen, politisches Handeln sei rational und beide, Politiker und Wähler, handelten im Eigeninteresse. Das Ziel des Wählers ist die Nettonutzenmaximierung, das des Politikers die Stimmenmaximierung. Folglich ähnelt der Wettbewerb der Politiker um Stimmen dem Wettbewerb der Produzenten um die Konsumenten.

Der Sport, so die Ansicht des Autors, bietet nun ein geradezu prädestiniertes Feld für stimmenmaximierendes Politikerverhalten. So gibt es kaum weitere öffentliche Ausgaben, die einer ähnlich breiten Masse zugutekommen und den Staat so vergleichsweise wenig kosten. Man bedenke, daß mit sechs Milliarden DM über 20 Millionen Menschen gefördert werden; eine Relation, die ihresgleichen sucht.

Es liegt auf der Hand, daß Gemeindeparlamente - insbesondere in kleineren Kommunen - mit der grundsätzlichen Befürwortung und auch dem geschickt gewählten Zeitpunkt der Vergabe eines Investitionszuschusses an einen größeren Sportverein durchaus Stimmenfang betreiben können. Man stelle sich nur eine Kleingemeinde mit einem einzigen, größeren Sportverein vor, dem Bürgermeister und Gemeinderat ein halbes Jahr vor anstehenden Neuwahlen einen Zuschuß zu einem langersehnten Sportplatzneubau oder ähnlichem gewähren. Dabei dürften unterschiedliche Gesundheitswirkungen verschiedener Sportarten nicht so sehr relevant sein, entscheidend wird die Anzahl der zu gewinnenden Wählerstimmen sein.

Diese These kann zudem erhärtet werden, wenn man sich der qualitativen Aspekte der Sportförderung erinnert. Die neoklassischen, allokationstheoretischen Überlegungen hatten zum Ziel, jenes Ausmaß an Förderung zu bestimmen, das die volkswirtschaftlich optimale Nachfrage nach Sportgütern gewähren kann.

Unterstellt man, diese Nachfrage könne gröBtenteils mit der Bereitstellung kommunaler Sportstätten sowie der Vergabe von Investitionszuschüssen für den vereinseigenen Sportstättenbau realisiert werden. dann verbleibt die Frage nach der allokationstheoretischen Erklärung der restlichen direkten Zuwendungen an die Vereine wie Unterhaltungszuschüsse, Pro-Kopf-Förderungen, Fahrtgelder etc. Handelt es sich hierbei um Zuwendungen, die erforderlich sind, um ein bestimmtes Nachfrageniveau nach Sport-

${ }^{314} \mathrm{Vgl}$. Schumpeter, 1950.

${ }^{315}$ Vgl. Downs, 1968. 
gütern zu schaffen und aufrechtzuerhalten, oder stellen diese vielmehr eine politisch gewollte Kumulation der Geschenke an bestimmte Gruppen dar, für die es unter rein neoklassischen Gesichtspunkten wenig Anlaß gibt?

Wenngleich berücksichtigt werden muß, daß - normale Nachfrageverhältnisse unterstellt - mit einer ersatzlosen Streichung derartiger Gelder c.p. eine gewisse Nachfragereduzierung einhergeht, vermutet der Autor auch letzteres und vertritt die Ansicht, daß der Downs'sche Ansatz unverzichtbarer Bestandteil einer Erklärung der öffentlichen Sportförderpraxis darstellt. ${ }^{316}$

\subsection{Das Modell von Niskanen}

In einem bekannten und viel diskutierten Aufsatz hinterfragt Niskanen das Bürokratieverhalten und stellt unter anderem die Hypothese eines Budgetmaximierungsverhaltens von Bürokraten auf. ${ }^{317}$ Hierzu überträgt er die Charakteristika des gewinnmaximierenden, uneingeschränkt eigennützigen Geschäftsmanns auf den Bürokraten, welcher seiner These zufolge wie jedermann seinen Nutzen maximieren möchte. Die Nutzenmaximierung des Bürokraten besteht dabei in der Budgetmaximierung. Ausgabereduzierungen werden folglich ohnehin ausgeschlossen.

Gestützt wird diese Argumentation auf die Rationalität und den Überlebenswillen der Bürokraten. So sind die meisten Variablen, die die Motivstruktur des Bürokraten ausmachen, eine positive Funktion des Gesamtbudgets. Niskanen nennt als mögliche Variablen unter anderem Gehalt, Nebeneinkünfte aus dem Amt, öffentliches Ansehen und Macht. Wegen begrenzter Information und widerstreitender Interessen könne ein einzelner Bürokrat, so Niskanen weiter, unabhängig von seiner persönlichen Motivation gar nicht im öffentlichen Interesse handeln.

Der Überlebenswille des Bürokraten kommt schließlich darin zum Ausdruck, daß er den Ansprüchen der wesentlichen Einflußfaktoren auf seine Amtszeit, den Beschäftigten wie auch den Beamten der kollektiven Organisation genügen möchte. Erstere haben selbst ein Interesse an einem höheren Budget, bedeutet dies doch wiederum größere Sicherheit des Arbeitsplatzes ${ }^{318}$ und in aller Regel auch mehr Gehalt.

Nun zeigt bereits der Blick auf die kommunalen Sportförderrichtlinien eine Vielfalt an Einzelregelungen zur Mittelgewährung. Sportämter, die in ihrer Größenordnung im

\footnotetext{
${ }^{316}$ Es wäre unter diesem Aspekt darüber hinaus interessant, über einen längeren Zeitraum hinweg das Timing der Vergabe von Investitionszuschüssen in verschiedenen Kommunen zu untersuchen.

${ }^{317}$ Im Original, vgl. Niskanen, 1971. Die hier dargestellten Ausführungen beziehen sich auf Pommerehne/ Frey, 1979, S. 349 ff.

${ }^{318}$ Sind die Untergebenen des Bürokraten selbst verbeamtet, dann muß das Argument der größeren Arbeitsplatzsicherheit freilich relativiert werden.
} 
Betrachtungszeitraum im Vergleich zu den übrigen Sportausgaben überproportional zugenommen haben, könnten sicherlich mit bedeutend weniger Personal auskommen, gäbe es neben der Bereitstellung kommunaler Sportstätten und den Investitionszuschüssen zum vereinseigenen Sportstättenbau nicht diese Vielfalt an Fördermaßnahmen. Eine Entwicklung, die nicht nur ins Downs'sche Bild paßt, sondern gleichermaBen dem Modell von Niskanen entspricht.

Etliche Gespräche mit Sportamtsmitarbeitern verschiedener Kommunen haben darüber hinaus folgendes gezeigt: Einige Interviewpartner - primär in kleineren Kommunen wurden hellhörig, wenn sie vom Autor erfuhren, daß die Pro-Kopf-Ausgaben ihrer Kommune unter dem Bundesdurchschnitt lägen. Hieraus schlossen sie, hinsichtlich zukünftiger Haushaltsberatungen auf bessere Argumente zurückgreifen zu können.

Nun mag bei diesen Befragten die aus der ökonomischen Theorie der Bürokratie bekannte Hypothese einer Maximierung des Budgets aus Gründen der allgemeinen Reputation und der Hervorhebung der Bedeutung des eigenen Ressorts für derartige Aussagen verantwortlich sein, was allerdings nicht nachzuweisen ist. Auffallend häufig erweckten Sportamtsleiter oder -mitarbeiter jedoch den Eindruck, als würden sie ihre Funktion weniger als Verwalter, sondern vielmehr als Interessenvertreter des Sports einschätzen.

Interessenvertreter des Sports haben freilich dafür Sorge zu tragen daß es dem Sport möglichst gut geht und so erscheint auch dieses, vom Autor unterstellte Selbstverständnis einiger Sportverwalter sowie die starke Bindung zwischen Sportverwaltung und organisiertem Sport ein möglicher Erklärungsansatz dafür zu sein, daß die Sportausgaben möglicherweise höher sind, als sie rein neoklasssischen Überlegungen zufolge vielleicht sein sollten. Damit kommen die Ausführungen zum letzten Punkt der Erklärung öffentlicher Sportmittelvergabe in der Bundesrepublik Deutschland, den interessengruppentheoretischen Ansätzen.

\subsection{Sportförderung als Vereinsförderung -Interessengruppentheoretische Ansätze}

Der Charakter der öffentlichen Sportförderung als Vereinssportförderung und die gleichzeitige Nichtförderung jeglicher kommerzieller Sportangebote läßt sich mit den bislang dargestellten theoretischen Ansätzen nicht hinreichend erklären. Legt man abermals die gesundheits- und standortpolitische Bedeutung des Sports als ein Kriterium der Förderungswürdigkeit zugrunde und unterstellt ferner fühlbare positive externe Effekte, dann muß die weiter oben aufgeworfene und noch nicht beantwortete Frage, wieso bspw. ein kommerzieller Tennisanbieter im Gegensatz zu einem Tennis- 
verein keinerlei staatliche Fördergelder erhält, ein weiteres Mal in den Raum gestellt werden.

Es müssen folglich andere Erklärungsmuster dazu dienen, diese offenstehende Frage zu beantworten. Die ökonomische Theorie der Interessengruppen kann hierzu einen nützlichen Beitrag leisten. So können Olsons Logik kollektiven Handelns zufolge Interessengruppen ${ }^{319}$ einen Einfluß auf die politischen Entscheidungsträger ausüben, der auf verschiedenen Ursachen beruht und auf verschiedenen Kanälen vollzogen wird.

Im Zusammenhang mit dem Sport scheint insbesondere der Einflußfaktor Marktmacht von großer Bedeutung $\mathrm{zu}$ sein. ${ }^{320}$ Diese Marktmacht wiederum hängt unter anderem positiv ab von der Anzahl der Mitglieder und der Organisationsstruktur. In beiden Fällen handelt es sich um Faktoren, die vom kommerziellen Sport kaum, von den Sportverbänden hingegen zur Genüge erfüllt werden. Über 20 Millionen Mitglieder und die Organisation des Deutschen Sportbundes mögen dies belegen.

Was die Einflußkanäle betrifft, soll an dieser Stelle ein Verweis auf die weiter oben dargestellte Verstrickung von organisiertem Sport und sportpolitischen Entscheidungsträgern genügen. $\mathrm{Da} ß$ sich der organisierte Sport seiner Stärke bewußt ist und diese auch zu nutzen versucht, belegt eine Aussage seitens des Deutschen Sportbundes, angesichts verschiedener Kritik - unter anderem am Gemeinnützigkeitsstatus - eine neue Strategie zu eröffnen und die Vereine als Lobby in Bonn einzubeziehen. ${ }^{321}$

Es liegt auf der Hand, daß der Deutsche Sportbund als Interessenvertreter sämtlicher, ihm untergeordneter Fachverbände keinerlei Differenzierung zwischen verschiedenen Sportarten vornimmt und fühlbar unterschiedlichen Förderpraktiken entgegenwirken würde. Da auch Politikern und Bürokraten hieran kaum gelegen sein dürfte, läßt sich die geringe Differenzierung der Sportförderung nach verschiedenen Sportarten - die nicht zuletzt aufgrund derer unterschiedlich zu bewertender gesundheitlicher Auswirkungen angebracht wäre - polit-ökonomisch nachvollziehen. Forderungen nach einer stärkeren Differenzierung öffentlicher Sportförderpraktiken als auch nach Eindämmung laufender Maßnahmen dürften somit auf Widerstand von mehreren Seiten stoßen und politisch kaum durchzusetzen sein.

\footnotetext{
${ }^{319}$ Nicht alle Interessengruppen zeichnen sich durch eine Organisationsfähigkeit aus. So stellen gemeinsame wirtschaftliche Interessen lediglich eine notwendige, keinesfalls eine hinreichende Bedingung dar. Dies wird insbesondere hinsichtlich des Auftretens möglicher Trittbrettfahrerprobleme offenkundig. Möglichst kleine Gruppen, selektive Anreize oder ein Beitrittszwang werden als Bedingung für die Bildung und Aufrechterhaltung einer stabilen Interessengruppe genannt. Im Zusammenhang mit dem Sport entsteht diese Problematik kaum, da Ausschlußmöglichkeiten größtenteils gegeben sind.

${ }^{320}$ Weitere mögliche Einflußfaktoren sind Informationen, die sich die politischen Entscheidungsträger von den Interessengruppen erhoffen oder finanzielle Unterstützung der Parteien durch die Interessengruppen. Diesen Faktoren wird vom Autor im Zusammenhang mit dem Sport allerdings kaum Bedeutung beigemessen.

${ }^{321}$ Vgl. Deutscher Sportbund, 1990, S. 35.
} 
Zusammenfassend kann festgehalten werden, daß die Theorie der öffentlichen Güter durchaus einen Beitrag zur Beantwortung der Grundsatzfrage, "öffentliche Sportförderung - ja oder nein" leisten kann. Es zeigte sich jedoch, daß auch polit-ökonomische Ansätze, insbesondere zur Erläuterung verschiedener Details öffentlicher Sportförderung wie laufende Fördermaßnahmen, Vielfalt derselben oder einseitige Ausrichtung auf den organisierten Sport unabdingbar sind.

Weiterhin ungeklärt blieb bislang die Frage, wieso es fast ausschließlich die Kommunen sind, die sich in der Bundesrepublik Deutschland der Sportförderung annehmen. Die ökonomische Theorie des Föderalismus vermag hierauf Antworten zu geben. 


\section{Sportpolitik als Kommunalpolitik - Föderalismustheoretische Gedanken}

Eine der Hauptaussagen der ökonomischen Theorie des Föderalismus besteht in der Bestimmung einer unter allokationstheoretischen Gesichtspunkten sinnvollen Verteilung der öffentlichen Auf- und damit Ausgaben auf die verschiedenen Gebietskörperschaften eines Staates. Im Zusammenhang mit öffentlicher Sportförderung erscheinen darüber hinaus insbesondere Aussagen zur Theorie der Finanzzuweisungen von Bedeutung. ${ }^{322}$

\subsection{Theoretische Überlegungen zur Vermeidung und Internalisierung räumlicher externer Effekte}

Allokationstheoretischer Grundgedanke der Föderalismustheorie ist eine weitestgehende Vermeidung bzw. Internalisierung von spill-overs, d.h. räumlichen externen Effekten, um möglichen Ineffizienzen bei der Bereitstellung von öffentlichen Gütern zu begegnen. ${ }^{323}$

Eine Vermeidung von spill-overs kann zunächst dadurch erreicht werden, da $B$ man die jeweiligen Aufgaben und damit auch Ausgaben derart auf die verschiedenen Gebietskörperschaften eines politischen Gefüges oder Raumes verteilt, daß die aus der Bereitstellung von öffentlichen Gütern resultierenden Nutzen auch auf den geographischen Raum der jeweils bereitstellenden Gebietskörperschaft beschränkt sind.

Dies setzt eine Abgrenzung von Gebietskörperschaften in der Weise voraus, daß sich räumliche Nutzenerstreckung und bereitstellende Gebietskörperschaft geographisch exakt entsprechen.

Spill-overs variieren allerdings von Gut zu Gut. Wollte man also jegliche räumlichen externen Effekte mittels Schaffung entsprechender Gebietskörperschaften vermeiden, ${ }^{324}$ benötigte man für jedes öffentliche oder auch öffentlich bereitgestellte private Gut eine andere Gebietskörperschaftsgröße. Die Vorteile einer derartigen Vermeidung

\footnotetext{
${ }^{322}$ Weitere Aussagen trifft die Föderalismustheorie zur Verteilung der Einnahmen, zur Beantwortung der grundsätzlichen Frage der Vorteilhaftigkeit föderalistischer Systeme, zur effizienten Größe von Gebietskörperschaften und ähnlichem mehr. Vgl. hierzu u.a. Petersen, 1988, S. $112 \mathrm{ff}$.

${ }^{323}$ Der dahinter stehende Gedanke entspricht letztlich der bereits in Abschnitt 1.2.3.1. dargestellten Vermeidung suboptimaler Angebotsmengen im Falle des Vorliegens externer Effekte, hier allerdings übertragen auf räumliche Aspekte und öffentlich bereitgestellte Güter.

${ }^{324}$ Spill-overs können u.a. bedeuten, daß auch Bürger anderer Gebietskörperschaften an den Nutzen und Kosten der von einer bestimmten Gebietskörperschaft bereitgestellten öffentlichen Güter beteiligt werden, ohne jedoch am kollektiven politischen EntscheidungsprozeB in der anbietenden Gebietskörperschaft beteiligt zu sein. Man spricht in diesem Zusammenhang von interjurisdictional externalities, die es aus Gründen möglicher resultierender Ineffizienzen zu vermeiden gilt.
} 
von interjurisdictional externalities würden durch die Nachteile der Einrichtung etlicher Verwaltungseinheiten konterkariert werden.

Ein alternatives Mittel zur Internalisierung räumlicher externer Effekte stellen Finanzzuweisungen dar, die letztlich zu einer Nettofinanzierung des öffentlich bereitgestellten Gutes durch mehrere Gebietskörperschaften führen. Der hinter solchen Zweckzuweisungen stehende Gedanke ist einfach und entspricht letztlich jenem Mechanismus zur Internalisierung externer Effekte, die durch eine Subventionierung des betreffenden Gutes in Höhe der Emissionsquote $\ddot{E}$ erreicht werden kann. ${ }^{325}$

Investitionszuweisungen reduzieren c.p. die Kosten einer bestimmten Investitionsmaßnahme. Unterstellt man eine normale Nachfragekurve, so führt dies letztlich zu höheren Nettoinvestitionen. Zur Realisierung der optimalen Angebotsmenge sollten selbige derart bemessen sein, daß die Eigenbeteiligung die internen Nutzen in der bereitstellenden Gebietskörperschaft und die Finanzzuweisung die in der (den) anderen Gebietskörperschaft(en) anfallenden Nutzen widerspiegelt.

\subsection{Sportgüter und räumliche externe Effekte}

Kapitel III verdeutlichte zum einen den großen und immer noch steigenden Anteil kommunaler an den gesamten öffentlichen Sportausgaben, der 1991 ein relatives Niveau von - je nach Definition 80 bzw. $87 \%$ erreichte. Zum anderen konnte festgehalten werden, daß die Länder einen beträchtlichen Teil ihrer Sportmittel in Form von Zweckzuweisungen an Kommunen verausgaben.

Ohne in einer ähnlich ausführlichen Weise wie es innerhalb von Abschnitt 1.3.2.2. hinsichtlich der Existenz externer Effekte geschah, auch auf die räumliche Ausbreitung der Nutzen von Sportgütern einzugehen, ${ }^{326}$ sollen doch einige Aspekte aufgezeigt werden, die im Rahmen einer solchen Untersuchung zu berücksichtigen sind.

Zunächst stellt sich die Frage, wie sich spill-overs, seien diese nun Auswirkungen der öffentlichen Bereitstellung/ Subventionierung öffentlicher oder auch privater Güter, messen lassen. ${ }^{327}$

Grundsätzlich müßte eine derartige Messung dadurch erfolgen, daß man mittels empirischer Studien untersucht, in welchem Maße die Nutznießer von Sportangeboten zu-

${ }^{325} \mathrm{Vgl}$. hierzu Abschnitt 1.2.3.1.

326 Eine zufriedenstellende Beantwortung würde den Rahmen der Dissertation sprengen. Gleichwohl handelt es sich hierbei um einen Aspekt, der von nicht minderem Interesse ist und Gegenstand zukünftiger Untersuchungen im Bereich öffentlicher Sportförderung sein könnte.

${ }^{327}$ Im Zusammenhang mit räumlichen externen Effekten muß es sich keineswegs ausschließlich um Misch- oder gar öffentliche Güter handeln. Auch ein öffentlich bereitgestelltes, privates Gut kann spillovers erzeugen, indem seine Nutzenausdehnung die geographischen Grenzen der bereitstellenden Gebietskörperschaft überschreitet. 
gleich Bürger der fördernden Gebietskörperschaft sind. Somit wäre ein erster Ansatz zur Bestimmung einer spill-over-Quote gegeben. Nun sind mehrere Determinanten denkbar, die das Ausmaß räumlicher externer Effekte eines bestimmten Sportangebotes bestimmen. Eine pauschale Aussage zu treffen dürfte kaum möglich sein, vielmehr ist eine Trennung nach verschiedenen Kriterien erforderlich.

\subsubsection{Differenzierung nach Nutzenarten}

Bezüglich der internen Effekte gestaltet sich die Bemessung einer spill-over-Quote relativ leicht. So ließe sich bspw. stichprobenhaft die Herkunft der Nachfrager von Sportangeboten ermitteln.

Ähnlich proportional zur Herkunft der Nachfrager dürften sich - soweit vorhanden mögliche positive externe Effekte auf das Sozialverhalten der Nachfrager ausbreiten, während die in diesem Zusammenhang ebenfalls denkbaren negativen externen Effekte wie bspw. Zerstörungen und Ausschreitungen primär auf die bereitstellende Gebietskörperschaft beschränkt bleiben.

Völlig unabhängig von der Herkunft der Nachfrager erstrecken sich mögliche externe Gesundheitseffekte, die über Einsparungen im Gesundheitswesen letztlich gleichermaBen allen Bürgern eines Staates zugutekommen.

Etwas problematischer hingegen ist die Bewertung von spill-overs im Zusammenhang mit möglichen externen Effekten wie bspw. Standortvorteilen oder Werbeeffekten. Beschränken sich die resultierenden positiven Effekte nur auf die subventionierende Gebietskörperschaft oder profitiert das ganze Umland? Anhand eines konkreten Beispiels ausgedrückt: Profitiert nur die Freiburger Wirtschaft von möglichen Werbewerten des SC Freiburg oder profitiert daraus zugleich das Freiburger Umland oder der Tourismus im gesamten Schwarzwald und wenn ja, in welchem Maße?

\subsubsection{Differenzierung nach infrastrukturellen Gegebenheiten des Umlandes}

Ein wesentlicher Bestimmungsfaktor des Anteils auswärtiger Nachfrager und damit der Höhe der spill-over-Quote könnte im Ausmaß der Urbanisierung verschiedener Regionen liegen. Geht man bspw. von einem bestimmten Sportangebot in einer Kommune A aus, so hängen deren mögliche spill-overs auch vom Vorhandensein vergleichbarer Sportangebote im näheren Umland ab. Unterstellt man in Anlehnung an das 
Brecht'sche Gesetz ${ }^{328}$ eine Übernahme zentralörtlicher Funktionen in Großstädten, so liegt auf der Hand, daß diese Übernahmefunktion umso bedeutender ist, je ländlicher die nähere Umgebung der bereitstellenden Kommune A bzw. je geringer das Angebot an vergleichbaren Gütern im Umland.

Bezieht man dies auf das Beispiel Profifußball, so könnte daraus geschlossen werden, $\mathrm{da}$ die durch das Münchener Olympiastadion und den dort angesiedelten FC Bayern München entstehenden spill-overs auch aufgrund der ländlichen Umgebung Münchens größer sind als mögliche räumliche externe Effekte vergleichbarer Sportstätten im an Fußballbundesligaclubs reichen Rheinland oder Ruhrgebiet.

\subsubsection{Differenzierung nach verschiedenen Sportgütern}

Es ist anzunehmen, daß auch die Art verschiedener Sportgüter einen Einfluß auf die Herkunft der Nachfrager bzw. die spill-over-Quote hat, obschon dies zumindest indirekt wiederum eine Funktion der infrastrukturellen Gegebenheiten des Umlandes darstellen kann. Wenngleich weiter oben gezeigt wurde, daß sich die Kommunen bezüglich des Sportstättenbestands und der Höhe der Sportetats beträchtlich unterscheiden, so kann seit Auslaufen des Goldenen Plans dennoch von einer Grundversorgung zumindest mit Kernsportstätten gesprochen werden.

Es liegt nun nahe, daß die Nutzen aus dem Angebot von Kernsportstätten des Freizeitund Breitensports tendenziell stärker auf die bereitstellenden Gebietskörperschaften bzw. Kommunen beschränkt bleiben. Unterstellt man bspw., daß es in fast jeder deutschen Kommune einen Fußballverein gibt, dann wird offenkundig, daß die öffentliche Bereitstellung eines Fußballplatzes für den Amateurfußball kaum zu merklichen spillovers führen dürfte. Anders hingegen bei bestimmten Sondersportanlagen, die aufgrund von

Nachfrage-, räumlichen oder sonstigen Gesichtspunkten wesentlich weniger flächendeckend bereitgestellt werden. Es sei nicht zuletzt an die oben genannten Großstadien für den Zuschauersport, an Einrichtungen für den Wintersport und ähnliches gedacht. Hierbei handelt es sich um Sportstätten, die tendenziell stärkere spill-overs produzieren als sie von herkömmlichen Fußballplätzen oder Turnhallen zu erwarten sind. Insbesondere die mit Zuschauersportanlagen verbundenen Nutzen dürften zu einem beträchtlichen Anteil auch außerhalb der bereitstellenden Kommune Nutzen stiften. Gleiches gilt schließlich für Einrichtungen, die - wie Bundes- oder Landesleistungszentren - ex definitione nicht nur einer bestimmten Kommune oder Region dienen.

${ }^{328}$ Zum Brecht'schen Gesetz der progressiven Parallelität zwischen Ausgaben und Bevölkerungsmassienung, vgl. auch Kapitel V, Abschnitt 2.4. 
Wenngleich eine exakte Bewertung der in den jeweiligen Gebietskörperschaften anfallenden Nutzen nicht möglich ist, stellt die relativ problemlos durchführbare Ermittlung der Herkunft der Nachfrager nach Sportgütern zumindest eine brauchbare Annäherung dar, die spill-overs verschiedener Sportgüter zu ermitteln. Unterstellt man, daß ein Großteil der Nutzen von Sportgütern auf den geographischen Raum der bereitstellenden Gebietskörperschaft beschränkt bleibt, dann kann die Theorie des Föderalismus auch dazu beitragen, den primär kommunalen Charakter öffentlicher Sportförderung zu erklären.

Umgekehrt können die gleichwohl denkbaren spill-overs - insbesondere im Zusammenhang mit Sondersport- oder Zuschauersportanlagen sowie in schwächer besiedelten Regionen - die Existenz von Zweckzuweisungen als Mittel der Internalisierung räumlicher externer Effekte erklären.

Die optimale Höhe der Zweckzuweisungen kann freilich nur theoretisch bestimmt werden, eine Erklärung der in der Praxis gewährten Zweckzuweisungen ist aufgrund der Unmöglichkeit einer exakten Bewertung räumlicher externer Effekte nicht möglich.

Abschließend soll abermals auf die vielfach zitierte Kritik am Instrument der Investitionszuweisungen hingewiesen werden. Solche Gelder werden nicht nur auf dem Sportsektor häufig als Instrument der Unterwanderung kommunaler Ausgabeautonomie beanstandet. Es liegt auf der Hand, daß kommunale Entscheidungsträger nicht auf derlei "Geschenke" verzichten wollen und darüber hinaus möglicherweise der Blick für Folgekosten von Investitionen, die durch den Bund und primär die Länder mitinitiiert werden, verloren gehen kann.

Die in Kapitel V folgende Diskussion möglicher Bestimmungsfaktoren öffentlicher Sportbudgets wird sich auch mit der Frage beschäftigen, inwieweit die These von den Zweckzuweisungen als Instrument der Unterwanderung kommunaler Ausgabeautonomie für den Bereich des Sports zutrifft. 
Christian Hockenjos - 978-3-631-75159-6

Downloaded from PubFactory at 01/11/2019 07:36:54AM

via free access 


\section{Kap. V Bestimmungsfaktoren öffentlicher Sportbudgets}

\section{Vorbemerkung}

Die Darstellung der Entwicklung des Sportausgabeniveaus im Zeitablauf offenbarte neben fühlbaren Schwankungen der Investitionstätigkeit einen vergleichsweise konstanten Anstieg der laufenden Ausgaben. Im Rahmen der Querschnittsbetrachtungen konnten beachtliche Unterschiede zwischen den Ausgabevolumina verschiedener Gebietskörperschaften der selben Ebene festgestellt werden.

Wovon hängt es ab, wieviel Gelder eine Gebietskörperschaft für den Sport verausgabt, lautete demzufolge eine der am Ende von Kapitel III aufgeworfenen Fragen. Die bisherigen theoretischen Ausführungen gaben bereits erste Hinweise zur Beantwortung dieser Frage; nunmehr sollen diese Anhaltspunkte ergänzt und konkretisiert werden, um Aussagen über mögliche Bestimmungsfaktoren öffentlicher Sportbudgets abzuleiten. ${ }^{329}$

\section{Finanzielle Rahmenbedingungen}

Die Gewährung von Sportfördermitteln stellt eine freiwillige Aufgabe dar, was in $80 \%$ der analysierten Sportförderrichtlinien durch den Hinweis auf das Nichtvorhandensein von Rechtsansprüchen deutlich hervorgehoben wird. Der Umfang der Sportausgaben, so die Richtlinien weiter, hänge von der jeweiligen Haushaltslage ab.

Abseits jeglicher theoretischer Überlegungen lieferte somit die Betrachtung der Sportförderrichtlinien den ersten Hinweis auf einen möglichen Bestimmungsfaktor, die positive Abhängigkeit der Sportausgaben von der finanziellen Situation einer Kommune. 330

Ein Vergleich von aggregierten Sportausgaben und Steuereinnahmen im Zeitablauf wie auch die Gegenüberstellung der Sportbudgets einzelner Gebietskörperschaften mit ihren finanziellen Rahmenbedingungen zeigte allerdings, daß der vielfach proklamierten

\footnotetext{
${ }^{329}$ Entsprechend der Gewichtung in den vorangegangenen Abschnitten beschränkt sich die Analyse in diesem Kapitel auf eine Betrachtung der Sportfördenungs- und Sportstättenausgaben

${ }^{330} \mathrm{Als}$ Indikator für die finanzielle Situation sind mehrere Größen denkbar. Der Deutsche Sportbund selbst hat mit der weiter o.g. Empfehlung an die Kommunen, zumindest $0,5 \%$ des kommunalen Budgets für den Sport zu verausgaben, eine Orientierung am Gesamthaushalt ins Spiel gebracht. Gleichermaßen sind kommunale Nettosteuereinnahmen in Betracht zu ziehen, die sich in erster Linie aus den um die Gewerbesteuerumlage gekürzten Gewerbesteuereinnahmen sowie den Grundsteuern A und B und dem gemeindlichen Anteil an der Einkommensteuer zusammensetzen.

Vom Autor auf Bestimmungsfaktoren des Ausgabevolumens angesprochenen, nannten einige der interviewten Sportämter die Gewerbesteuereinnahmen. Von einer Orientierung des Sportbudgets am Gesamthaushalt wußte keiner der Befragten zu berichten.
} 
Bedeutung der finanziellen Rahmenbedingungen als möglichem Bestimmungsfaktor Grenzen gesetzt sind.

So konnten im Zusammenhang mit den Zeitreihenbetrachtungen der Sportinvestitionen zwar in einigen Perioden tendenzielle Parallelen zwischen konjunkturellem Verlauf und aggregiertem Ausgabegebaren festgestellt werden, ${ }^{331}$ gleichwohl vorhandene gegensätzliche Tendenzen ${ }^{332}$ sowie das weitaus größere Ausmaß der Schwankungen der Sportinvestitionsvolumina deuten allerdings auf zusätzliche Bestimmungsfaktoren mit höherem Gewicht hin.

Noch offensichtlicher wurde dieser Sachverhalt bei einer Betrachtung der laufenden Sportausgaben. Zeigten sich selbige im Zeitablauf von konjunkturellen Schwankungen kaum beeinflußt, so belegen insbesondere die in den Abbildungen 17 und 22 dargestellten beachtlichen Unterschiede zwischen den Ausgabeniveaus verschiedener Gebietskörperschaften im Querschnitt, daß die Höhe der laufenden Sportausgaben auf andere Bestimmungsfaktoren zurückzuführen ist. ${ }^{333}$

Wenngleich ein Einfluß der Finanzsituation damit keinesfalls uneingeschränkt negiert werden soll, muß es doch weitere Größen geben, die einen starken Einfluß auf das Ausgabeverhalten ausüben. Die folgenden Ausführungen werden diese Aussage nachhaltig unterstreichen.

\section{3. (Sport)-politische Prioritäten}

Öffentliche Investitionen in Sportstätten können ungeachtet aktueller finanzieller Spielräume hohe politische Priorität erlangen und das Ausmaß der Sportausgaben vorübergehend stark aufstocken. $\mathrm{Zu}$ denken ist in diesem Zusammenhang an langfristige sportpolitische Planungen bzw. Infrastrukturkonzepte, sportliche Ereignisse von großer internationaler Bedeutung oder auch Situationen kurzfristig entstehenden Handlungsbedarfs in Folge von Nachfrageverschiebungen.

Nicht zuletzt das bis dahin einmalige und flächendeckende Sportstätteninfrastrukturprogramm, der von seiten des organisierten Sports initiierte Goldene Plan, kann wesentlich dazu beitragen, den Anstieg der Investitionsausgaben in den 60er und 70er Jahren zu erklären. Es sei an dieser Stelle nur auf die in diesen beiden Jahrzehnten

${ }^{331}$ So z.B. zu Beginn der 80er Jahre.

${ }^{332}$ So $z . B$. Anfang bis Mitte der 70er Jahre

${ }^{333} \mathrm{Zu}$ den kommunalen Steuereinnahmen im Querschnitt sei auf die Veröffentlichungen des Deutschen Städtetages im Statistischen Jahrbuch Deutscher Gemeinden verwiesen, zu den aggregierten Steuereinnahmen im Zeitablauf, vgl. u.a. die vom Statistischen Bundesamt ebenfalls jährlich veröffentlichten Statistischen Jahrbücher. 
vielerorts geschaffene Grundausstattung an sportspezifischer Infrastruktur in Form von Sporthallen, Mehrzweckstadien oder Badeanstalten verwiesen.

Umgekehrt vermag die weitgehende Schließung dieser ursprünglich in den DOGRichtlinien zum Ausdruck gebrachten Bedarfslücke an sportlicher Infrastruktur neben der sich gleichzeitig verschlechternden konjunkturellen Lage den Einbruch öffentlicher Investitionstätigkeit zu Beginn der 80er Jahre (vgl. Abbildung 12) verständlich machen.

In einem ähnlichen Kontext sind die Ausgaben im Zusammenhang mit den olympischen Spielen 1972 in München - die insbesondere den Sporthaushalt des Bundes (vgl. Abbildung 23) vorübergehend vervielfachten - sowie die hohen Sportstätteninvestitionen des Haushaltsjahres $1974 \mathrm{zu}$ sehen (vgl. Abbildung 12), die in erster Linie auf die Baumaßnahmen im Zusammenhang mit der Ausrichtung der Fußballweltmeisterschaft 1974 zurückzuführen sind.

Jüngere Beispiele stellen unter anderem die Maßnahmen zum Aus- und Umbau der Arenen in Stuttgart, Karlsruhe und Freiburg dar. Insbesondere im Zusammenhang mit der Erweiterung des Freiburger Dreisamstadions sind nachfragebedingte Erklärungsschemata heranzuziehen, die dazu führten, daß eine finanziell bedeutsame sportpolitische Entscheidung unabhängig von langfristigen sportpolitischen Planungen und gegenwärtigen finanziellen Gegebenheiten recht kurzfristig getroffen wurde. ${ }^{334}$

Zusammenfassend kann festgehalten werden, daß Entscheidungen zugunsten eines größeren Investitionsprojekts nicht zwangsläufig an die finanzielle Situation der investierenden Gebietskörperschaft in der (den) Periode(n) der Erstellung gebunden sein müssen. Denkbar ist allerdings durchaus, daß sich bauliches Ausmaß und Ausstattung an den finanziellen Rahmenbedingungen orientieren oder daß - bspw. im Zusammenhang mit langfristig geplanten Sportstätteninfrastrukturmaßnahmen - Entscheidungen über den konkreten Zeitpunkt der Durchführung einer Investition und damit über die Periode(n) der Mittelverwendung in Abstimmung mit weiteren geplanten, den finanziellen Handlungsspielraum einschränkenden sportfremden Investitionen getroffen werden.

\footnotetext{
${ }^{334}$ Die neuerlichen Zuwendungen des Landes Baden-Württemberg an die Stadt Freiburg zum weiteren Ausbau des Freiburger Dreisamstadions wurden vom derzeitigen baden-württembergischen Ministerpräsidenten Teufel nicht zuletzt mit der großen Zuschauerresonanz begründet, die der SC Freiburg in seiner ersten Bundesligasaison erfuhr. Vgl. Badische Zeitung vom 11.05.1994, " Stadionausbau - Das Land gibt fünf Millionen".
} 


\section{Zweckzuweisungen von Bund und Ländern}

Einen speziell im Zusammenhang mit gemeindlichen Sportausgaben stehenden Aspekt stellen die im Rahmen der föderalismustheoretischen Überlegungen erläuterten möglichen Auswirkungen investitionsorientierter Zweckzuweisungen von Bund und Ländern für kommunale Sportstätteninvestitionen dar. Der Vergleich von Sportinvestitionsvolumen und jeweiliger Höhe der Investitionszuweisungen seitens anderer Gebietskörperschaftsebenen konnte die vermuteten Beziehung nicht widerlegen, insbesondere auf einzelwirtschaftlicher Ebene ${ }^{335}$ zeigten sich mitunter deutliche Parallelen zwischen den betrachteten Größen

\section{Sportausgaben als Fixum - Folgekosten und polit-ökonomische Gesichts- punkte}

Die Ausführungen in Kapitel III konnten einen auffallend kontinuierlichen Anstieg der laufenden Sportausgaben im Zeitablauf nachweisen, dessen Hintergründe an dieser Stelle hinterfragt werden sollen. $\mathrm{Zu}$ diesem Zweck werden die beiden verschiedenen Typen laufender Ausgaben, die indirekte Förderung in Form der Bereitstellung und Unterhaltung kommunaler Sportstätten sowie die direkten laufenden Gelder an Sportvereine, getrennt betrachtet.

Zunächst zum laufenden Unterhaltungsaufwand im Zusammenhang mit kommunalen Sportstätten. Diese in Kapitel III, Abschnitt 2.1.4.2. näher erläuterten Kosten setzen sich zusammen aus Aufwendungen für die Bewirtschaftung der Grundstücke und baulichen Anlagen. Grundsätzlich handelt es sich dabei um Ausgaben, die keine größeren Unregelmäßigkeiten erwarten lassen. So unterliegen bspw. Personal- oder Energiekosten im Zeitablauf kaum größeren Schwankungen.

Hat eine Kommune erst einmal eine Sportstätte erstellt, gestaltet es sich recht problematisch, sich von den Folgekosten zu befreien. Sportstätten erfordern nun einmal einen gewissen Unterhaltungsaufwand, welcher - möchte man die Bausubstanz der jeweiligen Anlage und auch den Sportbetrieb aufrecht erhalten - unumgänglich ist.

Rein theoretisch ist zwar eine Reduzierung dieser laufenden Ausgaben durch Übertragung der Sportstätten auf die Nutzer möglich; dieser Ausweg ist aber insbesondere dort kaum möglich, wo es sich um Mehrzweckanlagen handelt, die von verschiedenen

${ }^{335}$ So u.a. in Karlsruhe, wo die starke Aufblähung des Vermögenshaushalts in den Jahren des Baus der Europahalle sowie der Modernisienung des Wildparkstadions mit hohen Zweckzuweisungen seitens des Landes Baden-Württemberg einherging. Vgl. Haushaltspläne der Stadt Karlsruhe. 
Sportgruppen bzw. Vereinen genutzt werden. ${ }^{336}$ Eine Zwangsübertragung ist ohnehin ausgeschlossen und es werden sich kaum zur Übernahme Bereitwillige finden, wenn eine Übernahme mit der Auflage versehen ist, etlichen anderen Gruppierungen eine Mitbenutzung zu gewähren.

Die weitere Möglichkeit, über Einnahmesteigerungen in Form erhöhter Benutzungsgebühren, wenngleich nicht die Nettoausgaben, so aber doch den Zuschußbedarf zu senken, scheint eher im Bereich des Realisierbaren zu liegen und wird in jüngster Zeit auch verstärkt diskutiert. ${ }^{337}$ Auch diesbezüglich haben die Financiers des Sports allerdings mit starkem Widerstand zu kämpfen. So versucht der organisierte Sport, geplante Gebührenerhebungen oder Ausgabekürzungen zu verhindern oder zumindest einzudämmen, ${ }^{338}$ womit merkliche Einsparungen erheblich erschwert werden.

Ähnliches gilt schließlich für die Höhe der laufenden direkten Zuwendungen. Im Zusammenhang mit geplanten Kürzungen dieses quantitativ weniger gewichtigen Ausgabetyps dürfte gleichermaßen mit Widerstand seitens der Empfänger zu rechnen sein, was die politische Durchsetzbarkeit fühlbarer Kürzungen ebenfalls erschwert.

Darüber hinaus stellt sich die Frage, inwieweit merkliche Änderungen bzw. Kürzungen derartiger Gelder überhaupt politisch gewollt sind. Unterstellt man der Theorie Niskanens zufolge ein gewisses Beharrungsverhalten der Sportbürokraten, läßt sich hieraus eine gewisse Konstanz sowohl der indirekten als auch der direkten laufenden Förderung ableiten. In die gleiche Richtung zielen schließlich Überlegungen auf der Grundlage von Downs' Stimmenmaximierungshypothese, derzufolge es kaum im Sinne eines vor seiner Wiederwahl stehenden Bürgermeisters sein dürfte, einen Teil seiner Wählerschaft zu vergraulen.

Neben den dargelegten Gründen für Inflexibilitäten von Sportausgaben im Verwaltungshaushalt stellt sich die Frage, ob gewisse Verstetigungstendenzen aufgrund von Beharrungsverhalten und ähnlichem möglicherweise auch im Zusammenhang mit Sportinvestitionen zu erwarten sind. Kapitel III zeigte, daß die Trendausreißer in der langfristigen Entwicklung der Sportausgaben auf Schwankungen im Investitionsverhalten zurückzuführen sind; die Investitionen sich also gerade nicht durch Konstanz auszeichneten. Nichtsdestotrotz gibt es keinen einsichtigen Grund, wieso ein "budgetmaximierender Bürokrat" nicht auch im Zusammenhang mit Investitionsausga-

\footnotetext{
${ }^{336} \mathrm{Im}$ Falle nicht-multifunktioneller Sportstätten ist eine derartige Übertragung der Anlage an einen einzigen Verein eher möglich, wie das Beispiel der Entwicklung der Trägerschaft von Sportplätzen in Nordrhein-Westfalen zeigt. Vgl. Kapitel II, Abschnitt 2.

${ }^{337} \mathrm{Vgl}$. Kapitel III, Abschnitt 2.1.6.4.

${ }^{338} \mathrm{Vgl}$. u.a. Frankfurter Allgemeine Zeitung vom 17.05.1993, "Wird die soziale Offensive zur unsozialen Defensive? - 42. Hauptausschußsitzung des Deutschen Sportbundes/ Kämpferischer Hans Hansen" oder Mittelbayerische Zeitung vom 20.04.1994, "Sport bleibt eine Wachstumsbranche - Sechster Teil der Rezessions-Serie".
} 
ben zumindest bestrebt sein sollte, diesen Teil des Budgets gleichermaßen zu erhöhen, auf hohem Niveau zu halten oder doch wenigstens Reduzierungen zu verhindern. Eine nach Sportstätten- und Sportförderungsinvestitionen differenzierende Betrachtung ist abermals angebracht.

Investitionen in die Sportstätteninfrastruktur zeichnen sich mitunter durch beachtliche Größenordnungen aus, so bspw. wenn eine Gemeinde ein größeres Stadion, eine Sporthalle oder ein Schwimmbad errichtet. Nun wäre es allerdings vermessen, dem Faktor Beharrung derart hohes Gewicht beizumessen und zu erwarten, daß eine Großinvestition in einer Periode $\mathbf{t}$ aufgrund eines politischen Beharrungsverhaltens $\mathrm{zu}$ ähnlich hohen Investitionen in den folgenden Perioden führt.

Etwas anders kann im Zusammenhang mit einmaligen Ausgaben innerhalb des Haushaltsabschnitts 550 argumentiert werden. Im Gegensatz zu den Investitionen in kommunale Sportstätten setzt sich dieser Haushaltsabschnitt in etlichen Kommunalbudgets aus mehreren einzelnen, kleineren Investitionszuschüssen zusammen. Eine gewisse Verstetigung dieser Investitionszuschüsse, die auch als "laufende Investitionsausgaben" angesehen werden können, ist denkbar. Diese Vermutung wird insbesondere dann gestärkt, wenn Kommunen für Sportförderungsinvestitionen jährliche Pauschalbeträge festgesetzt haben, wie es bspw. im Fall der Stadt Freiburg zutrifft. ${ }^{339}$ Überdies wird es grundsätzlich im Interesse örtlicher Stadtsportbünde wie auch der überregionalen Lobby des Sports liegen, einen jährlichen Topf für vereinseigene Investitionen sicherzustellen.

Die Überlegungen dieses Abschnitts zeigen, daß es gute Gründe für die dargestellte Inflexibilität insbesondere der laufenden Sportausgaben gibt.

Das finanzielle Ausmaß der laufenden Ausgaben zur Unterhaltung kommunaler Sportstätten hängt primär vom jeweiligen Sportstättenbestand ab und ist aufgrund des zwingend anfallenden Unterhaltungsaufwands sowie zu erwartender Widerstände seitens der Nutzer kurzfristig kaum merklich zu verändern. Ähnliches gilt für direkte Fördergelder an Sportvereine. Diese Gelder sind zwar grundsätzlich flexibler zu steuern als der oben genannte Unterhaltungsaufwand, abermals politische Widerstände dürften jedoch fühlbare Schwankungen bzw. Reduzierungen zumindest erschweren.

${ }^{339} \mathrm{Vgl}$. Haushaltspläne der Stadt Freiburg ab 1986 


\section{Die Subjektivität der Bewertung von Sportgütern}

Zum Abschluß der Diskussion möglicher Bestimmungsfaktoren soll auf einen in $\mathrm{Zu}$ sammenhang mit der Theorie der öffentlichen Güter stehenden Aspekt hingewiesen werden.

So tragen die unüberwindbaren Probleme im Zusammenhang mit der Bewertung externer Effekte sowie die mit der Definition des Begriffs meritorische Güter einhergehenden Freiräume dazu bei, daß dem Sport abseits finanzieller Rahmenbedingungen von verschiedenen politischen Entscheidungsträgern unterschiedliche Bedeutung beigemessen wird. Es ist vorstellbar, daß Politiker in einer Gebietskörperschaft A potentielle externe Effekte höher bewerten als in einer Gebietskörperschaft B oder daß der Sport grundsätzlich von einigen Entscheidungsträgern als förderungswürdig (meritorisch), von anderen als weniger förderungswürdig angesehen wird. ${ }^{340}$ Gleichermaßen ist $\mathrm{zu}$ vermuten, daß die Stärke der Lobby der Sportmittelempfänger einen Einfluß auf das Niveau der jeweiligen kommunalen Sportbudgets ausüben kann.

Derart subjektive Einschätzungen des Sports können freilich nicht operationalisiert werden, tragen nach Auffassung des Autors jedoch in starkem Maße dazu bei, die in Kapitel III dargestellten beachtlichen, mit finanziellen Rahmenbedingungen keineswegs zu erklärenden Unterschiede in den Pro-Kopf-Ausgabeniveaus verschiedener Kommunen wie auch verschiedener Bundesländer verständlich zu machen.

Zusammenfassend kann festgehalten werden, daß die Bestimmung des Umfangs öffentlicher Sportbudgets ein recht komplexes Phänomen darstellt. Die Ausführungen konnten zeigen, daß eine Reduzierung auf finanzielle Rahmenbedingungen keinesfalls möglich ist, daß vielmehr temporäre sportpolitische Prioritäten, die Erfordernisse der Unterhaltung bestehender Sportstätten, polit-ökonomische Aspekte und nicht zuletzt auch die subjektive Einschätzung des Gutes Sport eine gewichtige Rolle spielen.

\footnotetext{
${ }^{340} \mathrm{~W}$ as möglicherweise auch mit dem Sportinteresse der jeweiligen politischen Entscheidungsträger zusammenhängen mag. So ist doch keineswegs ausgeschlossen, daß ein sportbegeisterter Bürgermeister den Sport c.p. mehr fördert als sein nicht sportinteressierter Kollege aus der Nachbargemeinde.
} 
Christian Hockenjos - 978-3-631-75159-6

Downloaded from PubFactory at 01/11/2019 07:36:54AM

via free access 


\section{Kap. VI Resümee und Ausblick}

Öffentliche Sportförderung in der Bundesrepublik Deutschland zeichnet sich zum einen durch die Bereitstellung von Sportstätten (indirekte Sportförderung) und zum anderen durch die Vergabe von Zuschüssen (direkte Sportförderung) aus.

Das finanzielle Ausmaß dieses Typs staatlicher Subventionspolitik belief sich in den alten Bundesländern im Rechnungsjahr 1991 inklusive der Ausgaben für Badeanstalten auf etwa DM 6,1 MRD. Damit wird ein großer Teil der Kosten des Sporttreibens aus öffentlichen Mitteln finanziert.

Knapp $90 \%$ der von den Gebietskörperschaften verausgabten Gelder stammt aus Kommunalhaushalten. Empfänger sind fast ausschließlich die zugleich bedeutendsten Anbieter von Sportgütern, die unter dem Dach des Deutschen Sportbundes organisierten Sportvereine.

Die allokationstheoretische Analyse dieses staatlichen Eingriffs konnte das Vorliegen verschiedener externer Effekte nachweisen, die eine öffentliche Subventionierung prinzipiell verständlich machen, wenngleich sie nicht ihre absolute und relative Höhe erklären können. Es waren zusätzliche theoretische Ansätze erforderlich, um das Phänomen öffentliche Sportförderung, insbesondere die starke Ausrichtung auf den organisierten Sport, die Vielfalt der Fördermaßnahmen und auch die geringe Differenzierung nach verschiedenen Sportarten nachvollziehen zu können. Polit-ökonomische Ansätze konnten zum besseren Verständnis dieser Einzelaspekte öffentlicher Sportförderung wesentlich beitragen.

Die Analyse verdeutlichte ferner, daß sich Sportgüter größtenteils durch begrenzte Vorteile kollektiver Nutzung auszeichnen, welche wiederum ein Angebot durch einen Klub nahelegen. Die trotz dieser klubtheoretischen Überlegungen und der starken Wettbewerbsvorteile des organisierten Sports festzustellende Marktpräsenz alternativer, insbesondere kommerzieller Sportanbieter konnte mit gleichwohl vorhandenen preispolitischen Ineffizienzen der Sportvereine und einigen weiteren Charakteristika des organisierten Sports verständlich gemacht werden.

Es bleibt abzuwarten, wie sich die Angebotsstruktur von Sportgütern in der Zukunft entwickeln wird. Unterstellt man eine zunehmende Individualisierung der Gesellschaft, dann ist ein wachsendes Gewicht alternativer Sportanbieter denkbar.

Wenngleich sich die Gemeinden bezüglich der praktischen Ausgestaltung der Sportpolitik an Empfehlungen der kommunalen Spitzenverbände und auch der Sportorganisationen orientieren, kann von einer Nivellierung der Sportpolitik nicht die Rede sein. Sowohl die Betrachtung qualitativer als auch quantitativer Aspekte offenbarte erhebliche Unterschiede zwischen den verschiedenen sportpolitischen Trägern, die mit unter- 
schiedlichen Finanzausstattungen allein nicht zu erklären sind. Die mit der Bewertung externer Effekte und auch dem Aspekt der Meritorik einhergehenden Freiräume konnten dazu beitragen, die unterschiedlichen Ausgabeniveaus in den verschiedenen Gebietskörperschaften einer föderalen Ebene verständlich zu machen.

Die Entwicklung öffentlicher Sportausgaben seit 1965 zeichnet sich durch einen beachtlichen nominalen und auch realen Anstieg aus, der vorrangig auf einem kontinuierlichen Anwachsen der laufenden Ausgaben beruht. Entscheidungen über die Höhe dieses Typs öffentlicher respektive kommunaler Sportausgaben liegen nur bedingt in der Gewalt der jeweiligen Geldgeber. So verhindern Folgekostenzwänge und auch politische Widerstände eine flexiblere Handhabe laufender Sportausgaben.

Weitaus weniger konstant präsentierten sich die Investitionen, die ihren realen Höhepunkt bereits mit dem Jahr 1980 überschritten haben. Als Ursache der Schwankungen kommunaler Investitionen im Zeitablauf sind neben konjunkturellen Gegebenheiten in starkem Maße auch langfristige sportpolitische Planungen, wie sie bspw. im Goldenen Plan ihren Ausdruck fanden, anzusehen.

Das finanzielle Ausmaß öffentlicher Sportförderung wird nach Ansicht des Autors allen Sparvorhaben und -bemühungen zum trotz auch in Zukunft nicht oder zumindest nicht merklich reduziert werden, was primär in den Bestimmungsfaktoren der laufenden Aufwendungen begründet ist. Überdies bleibt abzuwarten, wie die sportpolitische Entwicklung in den neuen Bundesländern verlaufen wird. Der Deutsche Sportbund hat seine Vorstellungen mit der Konzeption des Goldenen Planes Ost bereits hinreichend konkretisiert. 


\section{Anhangverzeichnis}

Anhang 1: Der Aufbau des organisierten Sports in der BRD 169

Anhang 2: Laufende Ausgaben und Einnahmen des Karlsruher Wildparkstadions in $\mathrm{DM}$ 170

Anhang 3: Laufende Ausgaben und Einnahmen des Mönchengladbacher Bökelbergstadions in DM 172

Anhang 4: Die Sportausgaben der Gemeinden von 1965 bis 1991 in MIO DM 174

Anhang 5: Die Sportausgaben der Bundesländer von 1965 bis 1991 in MIO DM ... 178

Anhang 6: Die Sportausgaben des Bundes von 1965 bis 1991 in MIO DM 182

Anhang 7: Die Sportausgaben in der BRD von 1965 bis 1991 in MIO DM 184

Anhang 8: Die Sportausgaben der Stadt Freiburg von 1972 bis 1994 in DM 186

Anhang 9: Die Sportausgaben der Stadt Karlsruhe von 1972 bis 1994 in DM 187 
Christian Hockenjos - 978-3-631-75159-6

Downloaded from PubFactory at 01/11/2019 07:36:54AM

via free access 


\section{Anhang 1: Der Aufbau des organisierten Sports in der BRD}

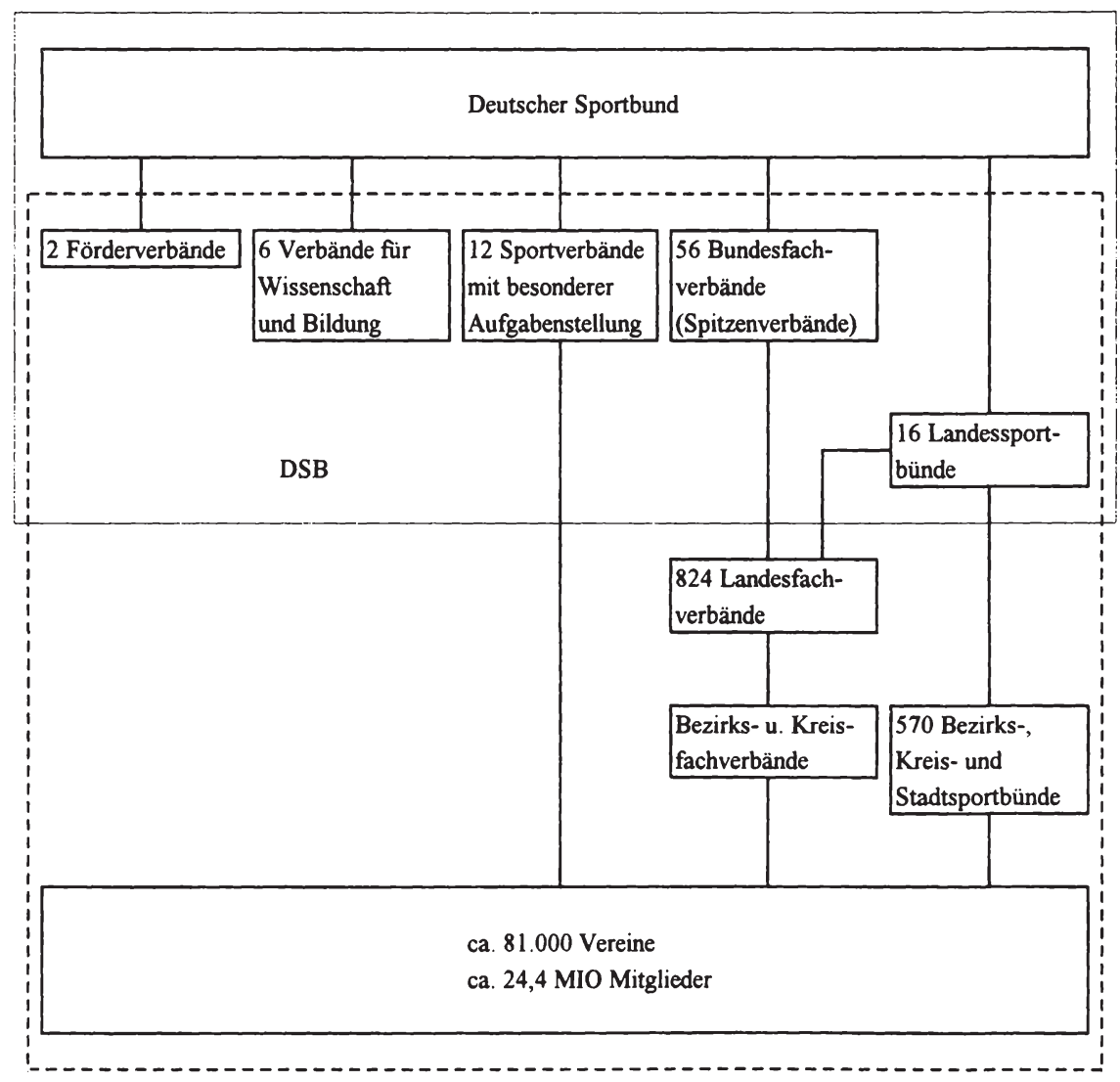

Quelle: Weber, 1995, S. 107. 


\section{Anhang 2: Laufende Ausgaben und Einnahmen des Karlsruher Wildpark- stadions in DM}

\begin{tabular}{|c|c|c|c|c|}
\hline Jahr & & 1977 & 1979 & 1981 \\
\hline Ausgaben & Gesamt & 281.213 & 332.313 & 547.918 \\
\hline darunter: & Personalausgaben & 143.394 & 160.382 & 199.793 \\
\hline & Unterhaltung der Grundstücke u. baul. Anlagen & 76.749 & 92.303 & 177.525 \\
\hline & Instandsetzung eigener Gebäude & -1.323 & 0 & -2.001 \\
\hline & Unterhaltung der Flutlichtanlage & 1.482 & 2.000 & 0 \\
\hline & Instandhaltung der Flutlichtanlage & 0 & & \\
\hline & Wartung der Anzeigetafel & & & \\
\hline & Unterhaltung der Sportanlagen & 30.762 & 41.137 & 124.439 \\
\hline & Arbeitsgeräte, Werkzeug und Maschinen & 3.233 & 2.590 & 5.642 \\
\hline & Reinigungsgeräte und -maschinen & & & 0 \\
\hline & Raumausstattung & 0 & 0 & 1.849 \\
\hline & Einrichtung & & 3.556 & 9.064 \\
\hline & Sportgeräte & & & \\
\hline & Mieten und Pachten & 1.944 & 0 & 0 \\
\hline & Mieten und Pachten für unbewegliches Vermögen & 6.700 & 7.700 & 6.700 \\
\hline & Bewirtschaftung der Grundstücke u. baul. Anlagen & 3.366 & 6.775 & 11.733 \\
\hline & Sonstige Bewirtschaftungskosten, Betriebskosten & & & \\
\hline & Dienst- und Schutzkleidung & 162 & 105 & 321 \\
\hline & Steuern, Schadensfälle, Versicherungen & 545 & 545 & 545 \\
\hline & Fernmeldegebühren & 400 & 420 & 980 \\
\hline & Dienstreisen & 0 & 0 & 169 \\
\hline & Besondere Verwaltungskostenbeiträge & & & 0 \\
\hline & Erstattung von Betriebsausgaben & 13.800 & 17.800 & 11.160 \\
\hline Einnahmen & Gesamt & 62.545 & 72.351 & 62.889 \\
\hline darunter: & Verkaufserlöse & 545 & 545 & 889 \\
\hline & Mieten und Pachten aus bebauten Grundstücken & 62.000 & 71.806 & 62.000 \\
\hline Ausgaben & & 281.213 & 332.313 & 547.918 \\
\hline Einnahmen & & 62.545 & 72.351 & 62.889 \\
\hline Zuschußbed: & & 218.668 & 259.963 & 485.029 \\
\hline Kostendecku & $\mathrm{g}$ (in \%) & 22 & 22 & 11 \\
\hline
\end{tabular}

Quelle: Stadt Karlsruhe, Haushaltsplåne, 1977 bis 1994. 


\begin{tabular}{|c|c|c|c|c|c|c|c|}
\hline 1983 & 1985 & 1987 & 1989 & 1991 & Ansatz 92 & Ansatz 93 & Ansatz 94 \\
\hline 443.487 & 463.680 & 608.923 & 535.824 & 631.980 & 693.590 & 665.780 & 697.300 \\
\hline 214.816 & 190.565 & 261.333 & 239.394 & 256.343 & 272.600 & 290.200 & 302.500 \\
\hline 59.530 & 61.063 & 74.812 & 48.216 & 95.900 & 103.840 & 106.050 & 106.050 \\
\hline 0 & 0 & 10.000 & & & & & \\
\hline \multirow[t]{3}{*}{6.816} & 9.188 & 1.032 & 9.510 & 6.910 & 30.000 & 10.000 & 30.000 \\
\hline & & & & 0 & 160.000 & 0 & 0 \\
\hline & 0 & 18.996 & 0 & & & & \\
\hline 95.800 & 106.335 & 96.174 & 107.087 & 129.254 & 102.570 & 85.000 & 85.000 \\
\hline 5.626 & 5.660 & 10.525 & 17.791 & 8.158 & 10.250 & 10.250 & 10.250 \\
\hline 0 & 0 & & & & & & \\
\hline 3.734 & 0 & & & 0 & 0 & 0 & 0 \\
\hline 0 & 0 & 677 & 0 & 0 & 1.000 & 1.000 & 1.000 \\
\hline 0 & 0 & 0 & 0 & 0 & 5.000 & 3.000 & 3.000 \\
\hline & & & & & & & \\
\hline 6.700 & 6.700 & 6.700 & 5.963 & 6.700 & 6.700 & 6.700 & 6.700 \\
\hline 19.357 & 36.725 & 70.818 & 50.633 & 35.439 & 55.530 & 39.900 & 40.450 \\
\hline 0 & 3.153 & 262 & 0 & 5.526 & 13.000 & 11.000 & 5.000 \\
\hline 683 & 577 & 49 & & 0 & 200 & 210 & 210 \\
\hline 545 & 545 & 545 & 599 & 0 & 690 & 2.000 & 2.000 \\
\hline 980 & 1.020 & 780 & 1.040 & 1.190 & 2.180 & 2.290 & 2.490 \\
\hline 0 & & & & & & & \\
\hline 28.900 & 28.900 & 30.370 & 31.630 & 59.060 & 61.470 & 67.960 & 71.380 \\
\hline 0 & 13.250 & 25.850 & 23.960 & 27.500 & 28.560 & 30.220 & 31.270 \\
\hline 90.600 & 86.543 & 145.496 & 114.169 & 113.778 & 111.690 & 220.500 & 262.000 \\
\hline 4.062 & 1.397 & 36.296 & 2.373 & 2.189 & 1.690 & 2.000 & 2.000 \\
\hline 94.661 & 87.940 & 109.200 & 111.796 & 111.589 & 110.000 & 218.500 & 260.000 \\
\hline 443.487 & 463.520 & 608.923 & 535.824 & 631.980 & 693.590 & 665.780 & 697.300 \\
\hline 94.661 & 87.940 & 109.200 & 114.169 & 113.778 & 111.690 & 220.500 & 262.000 \\
\hline 348.825 & 375.740 & 463.427 & 421.655 & 518.202 & 581.900 & 445.280 & 435.300 \\
\hline 21 & 19 & 18 & 21 & 18 & 16 & 33 & 38 \\
\hline
\end{tabular}




\section{Anhang 3: Laufende Ausgaben und Einnahmen des Mönchengladbacher Bökelbergstadions in DM}

\begin{tabular}{|c|c|c|c|c|c|}
\hline \multicolumn{2}{|l|}{ Jahr } & \multirow{2}{*}{\begin{tabular}{|l|}
1975 \\
917.331 \\
\end{tabular}} & \multirow{2}{*}{\begin{tabular}{|l|}
1977 \\
987.754 \\
\end{tabular}} & \multirow{2}{*}{\begin{tabular}{|l|}
1979 \\
117.650 \\
\end{tabular}} & \multirow{2}{*}{$\begin{array}{c}1981 \\
1.652 .491 \\
\end{array}$} \\
\hline Ausgaben & Gesamt & & & & \\
\hline \multirow[t]{15}{*}{ darunter: } & Personal & 49.760 & 62.470 & 68.040 & 70.350 \\
\hline & Grundstückunterhaltung & 27.350 & 47.600 & 28.430 & 38.000 \\
\hline & Instandhaltung Sportplätze & 35.408 & 13.000 & 19.258 & 18.520 \\
\hline & Sonstige Instandhaltung & 1.289 & 1.976 & 2.570 & 7.404 \\
\hline & Mieten und Pachten & & 0 & & \\
\hline & Bewirtschaftung der Grundstücke & 49.990 & 87.300 & 133.030 & \\
\hline & Bewachungskosten & 1.020 & 1.020 & 1.320 & 1.440 \\
\hline & Gutachter- u. ähnliche Kosten & & & & \\
\hline & Erstattung von Überzahlungen & & & & \\
\hline & Steuern und Versicherungen & 14.850 & 40.810 & 126.670 & \\
\hline & Sächliche Geschäftsausgaben & 0 & 0 & & \\
\hline & Vermischte Ausgaben & 30 & 0 & 0 & 0 \\
\hline & Innere Verrechnungen & 60.900 & 67.643 & 117.259 & 350.389 \\
\hline & Kalkulatorische Abschreibungen & 229.829 & 239.345 & 271.256 & 505.275 \\
\hline & Kalkulatorische Zinsen & 446.905 & 426.590 & 406.815 & 661.112 \\
\hline Einnahmen & Gesamt & 436.470 & 1.058 .259 & 2.298 .618 & 1.224 .670 \\
\hline \multirow[t]{7}{*}{ darunter: } & Miete & 348.331 & 733.228 & 474.809 & 611.339 \\
\hline & Verkaufsgenehmigungen & 62.410 & 74.218 & 131.952 & 139.390 \\
\hline & Nebenleistungen aus Mietverträgen & 14.220 & 23.269 & 37.977 & 25.026 \\
\hline & Reklameflächen & 0 & 193.417 & 516.617 & 325.752 \\
\hline & Vermischte Einnahmen & 1.685 & 334 & 0 & 0 \\
\hline & Mehrwertsteuer & 9.823 & 33.794 & 1.137 .262 & 123.162 \\
\hline & Erstattung von Aufwendungen & & & & \\
\hline \multicolumn{2}{|l|}{ Ausgaben } & 917.331 & 987.754 & 1.174 .650 & 1.652 .491 \\
\hline \multicolumn{2}{|l|}{ Einnahmen } & 436.470 & 1.058 .259 & 2.298 .618 & 1.224 .670 \\
\hline \multicolumn{2}{|c|}{ Zuschußbedarf } & 480.861 & -70.506 & 1.123 .968 & 427.821 \\
\hline \multicolumn{2}{|c|}{ Kostendeckung (in \%) } & 48 & 107 & 196 & 74 \\
\hline \multicolumn{2}{|c|}{ Kostendeckung ohne Mehrwertsteuer (in \%) } & 47 & 104 & 99 & 67 \\
\hline
\end{tabular}

Quelle: Stadt Mönchengladbach, Haushaltspläne, 1975 bis 1993. 


\begin{tabular}{|c|c|c|c|c|c|c|}
\hline 1983 & 1985 & 1987 & 1989 & 1991 & Ansatz 92 & Ansatz 93 \\
\hline 1.797 .156 & 2.142 .894 & 2.414 .041 & 2.431 .868 & 2.524 .972 & 3.085 .600 & 3.572 .100 \\
\hline \begin{tabular}{l|l}
65.210 \\
\end{tabular} & 50.580 & 154.800 & 160.400 & 197.468 & 203.900 & 221.500 \\
\hline & & & & & & \\
\hline 23.791 & 29.458 & 28.240 & 27.286 & 24.307 & 20.000 & 20.000 \\
\hline \multirow[t]{3}{*}{5.568} & 5.709 & 4.301 & 2.153 & 1.196 & 2.000 & 2.000 \\
\hline & & & & & & \\
\hline & & & & 0 & 0 & 0 \\
\hline \multirow[t]{5}{*}{1.440} & 1.680 & 1.560 & 1.560 & 1.560 & 1.500 & 1.600 \\
\hline & & & & 10.116 & & \\
\hline & & & & & & 140.000 \\
\hline & & & & & & \\
\hline & & & & & & \\
\hline 0 & 0 & & & & & \\
\hline 438.934 & 541.608 & 619.449 & 592.170 & 593.795 & 510.800 & 647.700 \\
\hline 521.885 & 581.797 & 643.198 & 696.096 & 770.948 & 784.800 & 857.100 \\
\hline 740.328 & 932.063 & 962.494 & 952.203 & 925.583 & 1.562 .600 & 1.682 .200 \\
\hline 1.316 .177 & 1.091 .204 & 1.769 .112 & 1.076 .018 & 1.290 .049 & 1.168 .100 & 1.398 .100 \\
\hline 358.867 & 297.691 & 350.077 & 269.232 & 405.374 & 280.000 & 590.000 \\
\hline 134.530 & 135.914 & 128.669 & 129.590 & 137.350 & 150.000 & 70.000 \\
\hline 20.523 & 37.680 & 33.934 & 32.284 & 34.803 & 38.000 & 38.000 \\
\hline 391.290 & 478.539 & 921.213 & 400.020 & 539.977 & 520.000 & 500.000 \\
\hline 101 & 88 & 0 & 0 & 0 & 100 & 100 \\
\hline \multirow[t]{2}{*}{410.867} & 141.293 & 335.220 & 192.917 & 172.546 & 180.000 & 200.000 \\
\hline & & 0 & 51.976 & & & \\
\hline 1.797 .156 & 2.142 .894 & 2.414 .041 & 2.431 .868 & 2.524 .972 & 3.085 .600 & 3.572 .100 \\
\hline 1.316 .177 & 1.091 .204 & 1.769 .112 & 1.076 .018 & 1.290 .049 & 1.168 .100 & 1.398 .100 \\
\hline 480.979 & 1.051 .690 & 644.929 & 1.355 .850 & 1.234 .923 & 1.917 .500 & 2.174 .000 \\
\hline 73 & 51 & 73 & 44 & 51 & 38 & 39 \\
\hline 50 & 44 & 59 & 36 & 44 & 32 & 34 \\
\hline
\end{tabular}




\section{Anhang 4: Die Sportausgaben der Gemeinden von 1965 bis 1991 in MIO DM}

\subsection{Ausgaben für Förderung des Sports und Sportstätten - nominal, getrennt nach}

\begin{tabular}{|c|c|c|c|c|c|c|c|c|c|c|c|}
\hline Jahr & 1965 & 1966 & 1967 & 1968 & 1969 & 1970 & 1971 & 1972 & 1973 & 1974 & 1975 \\
\hline Unmittelbare Ausgaben & 250 & 252 & 229 & 250 & 381 & 503 & 659 & 752 & 857 & 1.475 & 1.545 \\
\hline Zahlg. an offtl. Bereich & 18 & 16 & 18 & 20 & 25 & 35 & 44 & 56 & 60 & 73 & 68 \\
\hline Bruttoausgaben & 268 & 268 & 247 & 270 & 406 & 538 & 704 & 808 & 917 & 548 & 613 \\
\hline I. Zahlungen v. g & 3 & 3 & 3 & 4 & 5 & 7 & 11 & 11 & 11 & 29 & 41 \\
\hline Berei & 265 & 266 & 244 & 266 & 401 & 530 & 693 & 796 & 906 & 1.519 & 1.572 \\
\hline $1 . \mathrm{Zah}$ & 19 & 25 & 22 & 23 & 29 & 41 & 52 & 68 & 245 & 155 & 205 \\
\hline Netto: & 246 & 241 & 223 & 243 & 372 & 489 & 641 & 728 & 662 & 1.364 & 1.367 \\
\hline Unmitt & 20 & 27 & 31 & 31 & 38 & 69 & 63 & 78 & 104 & 148 & 122 \\
\hline wirtsch.Tätigkeit & & & & & & 7 & 8 & 9 & 11 & 20 & 22 \\
\hline Geb & 6 & 7 & 8 & 8 & 9 & 10 & 13 & 16 & 19 & 31 & 29 \\
\hline Zuschußbe & 226 & 214 & 192 & 212 & 334 & 420 & 578 & 650 & 558 & 1.216 & 1.245 \\
\hline Anteil der & 53,4 & 48,7 & 42,4 & 39,5 & 41,4 & $\overline{54,9}$ & 57,6 & 56 & 54,4 & $\overline{70,4}$ & $\overline{68,2}$ \\
\hline Anteil der lfd. Rechnung in \% & 46,6 & 51,3 & 57,6 & 60,5 & 58,6 & 45,1 & 42,4 & 44 & 45,6 & 29,6 & 31,8 \\
\hline
\end{tabular}

4.2. Ausgaben für Badeanstalten - nominal, getrennt nach Ausgabe- und Einnahmearten

\begin{tabular}{|c|c|c|c|c|c|c|c|c|c|c|c|}
\hline Jahr & 1965 & 1966 & 1967 & 1968 & 1969 & 1970 & 1971 & 1972 & 1973 & 1974 & 1975 \\
\hline Unmittelbare Ausgaben & 261 & 280 & 285 & 328 & 400 & 514 & 654 & 810 & 974 & 1.581 & 1.671 \\
\hline Zahlungen an öffl. Bereich & 2 & 2 & 3 & $\overline{3}$ & 4 & 7 & 11 & 10 & 7 & 44 & 46 \\
\hline Bruttoausgaben & 263 & 282 & 288 & 332 & 404 & 520 & 665 & 820 & 983 & 625 & 1.717 \\
\hline I. Zahlungen v. gleiche & 4 & 4 & 3 & 5 & 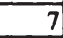 & 11 & 17 & 24 & 26 & 46 & 43 \\
\hline Bere & 259 & 279 & 285 & 326 & 398 & 510 & 648 & 796 & 957 & 1.578 & 1.674 \\
\hline $.1 . \mathrm{Zah}$ & 18 & 18 & 18 & 26 & 34 & 39 & 43 & 66 & 73 & 170 & 211 \\
\hline Netto & 241 & 261 & 267 & 300 & 364 & 471 & 605 & 729 & 884 & 1.408 & 1.463 \\
\hline Unmit & 60 & 72 & 98 & 98 & 120 & 140 & 171 & 199 & 244 & 300 & 386 \\
\hline Einn. aus wirtsch.Tätigkeit & 2 & 3 & 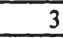 & 4 & 6 & 5 & 6 & & 9 & 14 & 18 \\
\hline tgelte & 47 & 58 & 79 & 73 & 95 & 102 & 125 & 139 & 168 & 232 & 312 \\
\hline Zuschußbec & 181 & 189 & 169 & 202 & 244 & 331 & 434 & 530 & 640 & 1.108 & 1.077 \\
\hline Anteil der Kapita & 51 & 48,7 & 42,8 & 41,7 & 46,5 & 49,6 & 51,1 & 52,5 & 50,4 & 56,5 & 49,5 \\
\hline Anteil der Ifd. Rechnung in \% & 49 & 51,3 & 57,2 & 58,3 & 53,5 & 50,4 & 48,9 & 47,5 & 49,6 & 43,5 & 50,5 \\
\hline
\end{tabular}

\subsection{Gesamte Nettosportausgaben - nominal, getrennt nach Haushaltsabschnitten}

\begin{tabular}{|l|r|r|r|r|r|r|r|r|r|r|r|}
\hline Jahr & $\mathbf{1 9 6 5}$ & $\mathbf{1 9 6 6}$ & $\mathbf{1 9 6 7}$ & $\mathbf{1 9 6 8}$ & $\mathbf{1 9 6 9}$ & $\mathbf{1 9 7 0}$ & $\mathbf{1 9 7 1}$ & $\mathbf{1 9 7 2}$ & $\mathbf{1 9 7 3}$ & $\mathbf{1 9 7 4}$ & $\mathbf{1 9 7 5}$ \\
\hline Förderung des Sports & 69 & 67 & 62 & 68 & 104 & 137 & 179 & 204 & 185 & 378 & 326 \\
\hline Sportstätten & 177 & 174 & 161 & 175 & 268 & 352 & 462 & 524 & $\mathbf{4 7 7}$ & 986 & 1.041 \\
\hline Förd. des Sports u. Sportstätten & 246 & 241 & 223 & 243 & 372 & 489 & 641 & 728 & 662 & 1.364 & 1.367 \\
\hline Badeanstalten & 241 & 261 & 267 & 300 & 364 & 471 & 605 & 729 & $\mathbf{8 8 4}$ & 1.408 & 1.463 \\
\hline Nettosportausgaben insgesamt & 487 & 502 & 490 & 543 & 736 & 960 & 1.246 & 1.457 & 1.546 & 2.772 & 2.830 \\
\hline
\end{tabular}




\begin{tabular}{|c|c|c|c|c|c|c|c|c|c|c|c|c|c|c|c|}
\hline 1976 & 1977 & \begin{tabular}{|l|}
1978 \\
\end{tabular} & \begin{tabular}{|l|}
1979 \\
\end{tabular} & \begin{tabular}{|l}
1980 \\
\end{tabular} & \begin{tabular}{|l|l}
1981 \\
\end{tabular} & \begin{tabular}{|l|l|}
1982 \\
\end{tabular} & 1983 & 1984 & \begin{tabular}{|l|}
1985 \\
\end{tabular} & \begin{tabular}{|l|}
1986 \\
\end{tabular} & \begin{tabular}{|l|}
1987 \\
\end{tabular} & \begin{tabular}{|l|l}
1988 \\
\end{tabular} & \begin{tabular}{|l|}
1989 \\
\end{tabular} & \begin{tabular}{|l|}
1990 \\
\end{tabular} & \begin{tabular}{|l|}
1991 \\
\end{tabular} \\
\hline 1.588 & 1.600 & 1.858 & 2.140 & 2.552 & 2.702 & 2.486 & 2.278 & 2.193 & 2.320 & 2.503 & 2.626 & 2.704 & 2.806 & 2.922 & 3.023 \\
\hline 67 & 64 & 63 & 73 & 91 & 102 & 88 & 69 & 67 & 65 & 70 & 64 & 61 & 59 & 67 & 62 \\
\hline 1.654 & 1.664 & 1.921 & 2.213 & 2.642 & 2.804 & 2.574 & 2.346 & 2.261 & 2.384 & 2.572 & 2.689 & 2.766 & 2.865 & 2.990 & 3.086 \\
\hline 47 & 45 & 43 & 56 & 64 & 85 & 77 & 59 & 62 & 57 & 65 & 67 & 61 & 56 & 63 & 58 \\
\hline 1.607 & 1.618 & 1.878 & 2.157 & 2.578 & 2.720 & 2.497 & 2.287 & 2.198 & 2.328 & 2.507 & 2.621 & 2.704 & 2.809 & 2.928 & 3.028 \\
\hline 249 & 168 & 206 & 228 & 240 & 254 & 241 & 209 & 193 & 201 & 197 & 210 & 207 & 197 & 213 & 212 \\
\hline 1.358 & 1.450 & 1.671 & 1.928 & 2.337 & 2.466 & 2.257 & 2.079 & 2.005 & 2.127 & 2.310 & 2.412 & 2.497 & 2.613 & 2.715 & 2.815 \\
\hline 139 & 158 & 167 & 206 & 244 & 259 & 264 & 262 & 260 & 267 & 279 & 287 & 278 & 304 & 310 & 310 \\
\hline 27 & 32 & 33 & 36 & 42 & 47 & 49 & 57 & & & & & & & & \\
\hline 32 & 36 & 38 & 43 & 47 & 50 & 57 & 61 & 64 & 66 & 70 & 73 & 80 & 80 & 84 & 85 \\
\hline 1.219 & 1.292 & 1.504 & 1.722 & 2.093 & 2.207 & \begin{tabular}{|l|} 
\\
\end{tabular} & 1.817 & 1.745 & 1.860 & 2.031 & 2.125 & 2.219 & 2.309 & 2.405 & 2.505 \\
\hline 65 & 60,8 & 60,9 & 61,3 & 62,1 & 61,3 & 56,5 & 50,8 & 44,7 & 44,2 & 46,1 & 46,9 & 47,2 & 46,7 & 45,6 & 44,4 \\
\hline 35 & 39,2 & 39,1 & 38,7 & 37,9 & 38,8 & 43,5 & 49,2 & 55,3 & 55,8 & 53,9 & 53,1 & 52,8 & 53,3 & 54,4 & $\overline{55}$ \\
\hline
\end{tabular}

\begin{tabular}{|l|l|l|l|l|l|l|l|l|l|l|l|l|l|l|l|}
\hline 1976 & 1977 & 1978 & 1979 & 1980 & 1981 & 1982 & 1983 & 1984 & 1985 & 1986 & 1987 & 1988 & 1989 & 1990 & 1991 \\
\hline
\end{tabular} \begin{tabular}{|r|r|r|r|r|r|r|r|r|r|r|r|r|r|r|r|}
\hline 1.580 & 1.455 & 1.461 & 1.586 & 1.799 & 1.859 & 1.810 & 1.756 & 1.723 & 1.736 & 1.794 & 1.814 & 1.850 & 1.947 & 2.091 & 2.178 \\
\hline 43 & 42 & 38 & 40 & 44 & 46 & 40 & 39 & 39 & &
\end{tabular} \begin{tabular}{|r|r|r|r|r|r|r|r|r|r|r|r|r|r|r|r|}
\hline 43 & 42 & 38 & 40 & 44 & 46 & 40 & 39 & 39 & 37 & 36 & 34 & 34 & 43 & 36 & 36 \\
\hline
\end{tabular} \begin{tabular}{|r|r|r|r|r|r|r|r|r|r|r|r|r|r|r|r|}
\hline 1.623 & 1.497 & 1.500 & 1.626 & 1.843 & 1.905 & 1.851 & 1.795 & 1.762 & 1.773 & 1.829 & 1.848 & 1.884 & 1.990 & 2.127 & 2.215 \\
\hline
\end{tabular} \begin{tabular}{|r|r|r|r|r|r|r|r|r|r|r|r|r|r|r|r|}
\hline 34 & 30 & 32 & 32 & 35 & 32 & 30 & 23 & 24 & 21 & 24 & 32 & 22 & 27 & 27 & 25 \\
\hline
\end{tabular} \begin{tabular}{|r|r|r|r|r|r|r|r|r|r|r|r|r|r|r|r|}
\hline 1.588 & 1.467 & 1.468 & 1.594 & 1.808 & 1.873 & 1.821 & 1.772 & 1.738 & 1.752 & 1.805 & 1.816 & 1.861 & 1.963 & 2.100 & 2.190 \\
\hline
\end{tabular} \begin{tabular}{|r|r|r|r|r|r|r|r|r|r|r|r|r|r|r|r|}
\hline 160 & 109 & 93 & 98 & 109 & 92 & 90 & 69 & 56 & 43 & 46 & 42 & 47 & 52 & 67 & 69 \\
\hline
\end{tabular}

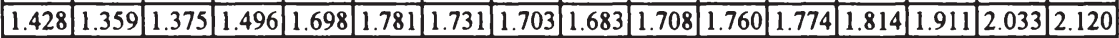

\begin{tabular}{|r|r|r|r|r|r|r|r|r|r|r|r|r|r|r|r|}
\hline 413 & 382 & 382 & 389 & 401 & 438 & 498 & 539 & 500 & 502 & 551 & 489 & 545 & 567 & 638 & 639 \\
\hline 21 & 21 & 23 & 24 & 25 & 28 & 31 & 35 & & & & & & & & \\
\hline 308 & 280 & 290 & 285 & 285 & 305 & 360 & 404 & 355 & 352 & 386 & 348 & 383 & 404 & 411 & 427 \\
\hline 1.015 & 977 & 993 & 1.107 & 1.297 & 1.343 & 1.233 & 1.164 & 1.183 & 1.206 & 1.209 & 1.285 & 1.269 & 1.344 & 1.395 & 1.481 \\
\hline 39,3 & 29,2 & 24,7 & 25 & 28 & 25,4 & 21,9 & 19,4 & 16,8 & 15,1 & 16,7 & 20,2 & 21,5 & 24 & 25,7 & 24,4 \\
\hline 60,7 & 70,8 & 75,3 & 75 & 72 & 74,6 & 78,1 & 80,6 & 83,2 & 84,9 & 83,3 & 79,8 & 78,5 & 76 & 74,3 & 75,6 \\
\hline
\end{tabular}

\begin{tabular}{|r|r|r|r|r|r|r|r|r|r|r|r|r|r|r|r|}
\hline 1976 & 1977 & 1978 & 1979 & 1980 & 1981 & 1982 & 1983 & 1984 & 1985 & 1986 & 1987 & 1988 & 1989 & 1990 & 1991 \\
\hline 346 & 383 & 449 & 527 & 627 & 673 & 655 & 611 & 621 & 646 & 679 & 708 & 724 & 756 & 821 & 861 \\
\hline 1.012 & 1.067 & 1.222 & 1.401 & 1.710 & 1.793 & 1.602 & 1.468 & 1.384 & 1.481 & 1.631 & 1.704 & 1.773 & 1.857 & 1.894 & 1.954 \\
\hline 1.358 & 1.450 & 1.671 & 1.928 & 2.337 & 2.466 & 2.257 & 2.079 & 2.005 & 2.127 & 2.310 & 2.412 & 2.497 & 2.613 & 2.715 & 2.815 \\
\hline 1.428 & 1.359 & 1.375 & 1.496 & 1.698 & 1.781 & 1.731 & 1.703 & 1.683 & 1.708 & 1.760 & 1.774 & 1.814 & 1.911 & 2.033 & 2.120 \\
\hline 2.786 & 2.809 & 3.046 & 3.424 & 4.035 & 4.247 & 3.988 & 3.782 & 3.688 & 3.835 & 4.070 & 4.186 & 4.311 & 4.524 & 4.748 & 4.935 \\
\hline
\end{tabular}


4.4. Gesamte Nettosportausgaben - nominal und real, Basisjahr 1985

\begin{tabular}{|l|r|r|r|r|r|r|r|r|r|r|r|}
\hline Jahr & 1965 & 1966 & 1967 & 1968 & 1969 & 1970 & 1971 & 1972 & 1973 & 1974 & 1975 \\
\hline nominal & 487 & 502 & 490 & 543 & 736 & 960 & 1.246 & 1.457 & 1.546 & 2.772 & 2.830 \\
\hline real & 1.068 & 1.064 & 1.023 & 1.120 & 1.487 & 1.879 & 2.320 & 2.574 & 2.560 & 4.298 & 4.137 \\
\hline
\end{tabular}

4.5. Kostendeckungsgrade bei verschiedenen Sportausgaben

\begin{tabular}{|l|r|r|r|r|r|r|r|r|r|r|r|}
\hline Jahr & 1965 & 1966 & 1967 & 1968 & 1969 & 1970 & 1971 & 1972 & 1973 & 1974 & 1975 \\
\hline Förd. des Sports und Sportstätten & 8,1 & 11 & 14 & 13 & 10 & 14 & 9,8 & 11 & 16 & 11 & 8,9 \\
\hline Badeanstalten & 25 & 28 & 37 & 33 & 32 & 30 & 28 & 27 & 28 & 21 & 26 \\
\hline
\end{tabular}

4.6. Investive bereinigte Sportausgaben (Kapitalrechnung) für Förderung des Sports,

\begin{tabular}{|l|r|r|r|r|r|r|r|r|r|r|r|}
\hline Jahr & 1965 & 1966 & 1967 & 1968 & 1969 & 1970 & 1971 & 1972 & 1973 & 1974 & 1975 \\
\hline Sportf. u. Sportstätten, nominal & 142 & 130 & 104 & 105 & 167 & 291 & 399 & 446 & 493 & 1.069 & 1.072 \\
\hline Sportf. u. Sportst. real & 311 & 275 & 217 & 216 & 337 & 569 & 743 & 788 & 816 & 1.657 & 1.567 \\
\hline Badeanstalten, nominal & 132 & 136 & 122 & 136 & 184 & 253 & 331 & 418 & 482 & 891 & 828 \\
\hline Badeanstalten, real & 289 & 288 & 255 & 280 & 372 & 495 & 616 & 739 & 798 & 1.381 & 1.211 \\
\hline Sport gesamt, nominal & 274 & 266 & 226 & 241 & 351 & 544 & 730 & 864 & 975 & 1.960 & 1.900 \\
\hline Sport gesamt, real & 601 & 564 & 472 & 497 & 709 & 1.065 & 1.359 & 1.527 & 1.614 & 3.039 & 2.778 \\
\hline
\end{tabular}

Quellen:

Statistisches Bundesamt, Finanzen und Steuern.

1965 - 69: Fachserie L, Reihe 5, Sonderbeiträge zur Finanzstatistik, Aufwendungen von Bund, Ländern und Gemeinden (GV) für Gesundheitspflege und Sport sowie Reihe 1, Haushaltswirtschaft von Bund, Ländern und Gemeinden, II. Jahresabschlüsse, Kommunalfinanzen.

1970 - 73: Fachserie L, Reihe 5, Sonderbeiträge zur Finanzstatistik, Ausgaben der öffentlichen Haushalte für Gesundheit, Sport und Erholung.

1974 - 79: Fachserie 14, Reihe 3.6, Rechnungsergebnisse der öffentlichen Haushalte für Gesundheit, Sport und Erholung.

Ab 1980: Fachserie 14, Reihe 3.5, Rechnungsergebnisse der öffentlichen Haushalte für soziale Sichenung und für Gesundheit, Sport und Erholung.

Statistisches Bundesamt, Statistische Jahrbücher. 
\begin{tabular}{|l|l|l|l|l|l|l|l|l|l|l|l|l|l|l|l|}
\hline 1976 & 1977 & 1978 & 1979 & 1980 & 1981 & 1982 & 1983 & 1984 & 1985 & 1986 & 1987 & 1988 & 1989 & 1990 & 1991 \\
\hline
\end{tabular}

\begin{tabular}{|l|l|l|l|l|l|l|l|l|l|l|l|l|l|l|l|}
\hline 2.786 & 2.809 & 3.046 & 3.424 & 4.035 & 4.247 & 3.988 & 3.782 & 3.688 & 3.835 & 4.070 & 4.186 & 4.311 & 4.524 & 4.748 & 4.935 \\
\hline 3.897 & 3.801 & 4.01 & 4.351 & 4.873 & 4.821 & 4.302 & 3.948 & 3.763 & 3.835 & 4.078 & 4.190 & 4.268 & 4.354 & 4.450 & 4.466 \\
\hline
\end{tabular}

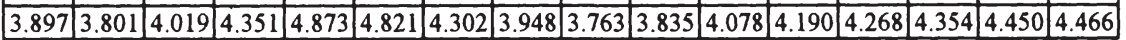

\begin{tabular}{|r|r|r|r|r|r|r|r|r|r|r|r|r|r|r|r|}
\hline 1976 & 1977 & 1978 & 1979 & 1980 & 1981 & 1982 & 1983 & 1984 & 1985 & 1986 & 1987 & 1988 & 1989 & 1990 & 1991 \\
\hline 10 & 11 & 10 & 11 & 10 & 11 & 12 & 13 & 13 & 13 & 12 & 12 & 11 & 12 & 11 & 11 \\
\hline 29 & 28 & 28 & 26 & 24 & 25 & 29 & 32 & 30 & 29 & 31 & 28 & 30 & 30 & 31 & 30 \\
\hline
\end{tabular}

Sportstätten und Badeanstalten, nominal und real, Basisjahr 1985

\begin{tabular}{|r|r|r|r|r|r|r|r|r|r|r|r|r|r|r|r|}
\hline $\mathbf{1 9 7 6}$ & $\mathbf{1 9 7 7}$ & $\mathbf{1 9 7 8}$ & $\mathbf{1 9 7 9}$ & $\mathbf{1 9 8 0}$ & $\mathbf{1 9 8 1}$ & $\mathbf{1 9 8 2}$ & $\mathbf{1 9 8 3}$ & $\mathbf{1 9 8 4}$ & $\mathbf{1 9 8 5}$ & $\mathbf{1 9 8 6}$ & $\mathbf{1 9 8 7}$ & $\mathbf{1 9 8 8}$ & $\mathbf{1 9 8 9}$ & $\mathbf{1 9 9 0}$ & $\mathbf{1 9 9 1}$ \\
\hline 1.045 & 983 & 1.143 & 1.322 & 1.601 & 1.666 & 1.410 & 1.161 & $\mathbf{9 8 2}$ & 1.030 & 1.156 & 1.229 & 1.277 & 1.311 & 1.334 & 1.343 \\
\hline 1.462 & 1.330 & 1.508 & 1.680 & 1.934 & 1.891 & 1.521 & 1.212 & 1.002 & 1.030 & 1.158 & 1.230 & 1.264 & 1.261 & 1.247 & 1.210 \\
\hline 624 & 428 & 363 & 399 & 507 & $\mathbf{4 7 5}$ & 398 & 344 & 292 & 265 & 302 & 367 & $\mathbf{4 0 1}$ & $\mathbf{4 7 2}$ & 540 & 534 \\
\hline $\mathbf{8 7 3}$ & 579 & 479 & 507 & 612 & 539 & $\mathbf{4 2 9}$ & 359 & 298 & 265 & 303 & 367 & 397 & $\mathbf{4 5 4}$ & 505 & $\mathbf{4 8 1}$ \\
\hline 1.669 & 1.411 & 1.506 & 1.721 & 2.108 & 2.141 & 1.808 & 1.505 & 1.274 & 1.295 & 1.458 & 1.596 & 1.678 & 1.783 & 1.874 & 1.877 \\
\hline 2.334 & 1.909 & 1.987 & 2.187 & 2.546 & 2.430 & 1.950 & 1.571 & 1.300 & 1.295 & 1.461 & 1.598 & 1.661 & 1.716 & 1.756 & 1.699 \\
\hline
\end{tabular}

\section{Erläuterungen:}

Explizite Unterscheidung zwischen Förderung des Sports und Sportstätten auf kommunaler Ebene erst ab 1974. Für die Haushaltsjahre 1965 bis 1973 wurden die jeweiligen Anteile eigenhändig errechnet, wozu sich der Autor an den durchschnittlichen Anteilen der Folgejahre orientierte. Der prozentuale Anteil an den Ausgaben für die Förderung des Sports an den gesamten Ausgaben variierte zwischen 25 und $31 \%$, der durchschnittliche Anteil lag bei $28 \%$.

Zum Kostendeckungsgrad: Hier definiert als (Unmittelbare Einnahmen : Nettoausgaben)* 100.

Zur Aufteilung in investive und laufende Ausgaben:

Expliziter Ausweis Kapital- vs. laufende Rechnung erst ab 1984 anhand der Bereinigten Ausgaben. Für die Haushaltsjahre 1970 bis 1983 wurde die Aufteilung (ebenfalls anhand der Bereinigten Ausgaben) eigenhändig errechnet. Zu diesem Zweck mußten die investiven bzw. laufenden Anteile der Zahlungen an den öffentlichen Bereich sowie der Zahlungen von gleicher Ebene geschätzt werden. Es wurde unterstellt, daß erstere zu 10, letztere zu 50\% investiver Natur sind. Diese Annahmen basieren auf den Ansätzen jener Jahre, die die investiven und laufenden Anteile der betreffenden Positionen ausweisen. Mögliche Abweichungen von den unterstellten Prozentsätzen sind insofern vernachlässigbar, als die quantitative Bedeutung der beiden betrachteten Positionen auf kommunaler Ebene ohnehin gering ist. Für die Jahre 1965 bis 1970 konnten die jeweiligen Anteile anhand der "Kommunalfinanzen" ermittelt werden. Um eine Vergleichbarkeit mit den anderen Veröffentlichungen herzustellen, mußten die innerhalb der Kommunalfinanzen ausgewiesenen Positionen Zinsen, Tilgung sowie Zuführung zu den Rücklagen herausgerechnet werden.

Leerzellen: Keine Angaben möglich, da kein Ausweis. 


\section{Anhang 5: Die Sportausgaben der Bundesländer von 1965 bis 1991 in MIO DM}

5.1. Gesamte Sportausgaben (Förderung des Sports, Sportstätten und Badeanstalten) -

\begin{tabular}{|c|c|c|c|c|c|c|c|c|c|c|c|}
\hline Jahr & 1965 & 1966 & 1967 & 1968 & 1969 & 1970 & 1971 & 1972 & 1973 & 1974 & 1975 \\
\hline Unmittelbare Ausgaben & 121 & 136 & 127 & 147 & 168 & 249 & 286 & 288 & 258 & 335 & 364 \\
\hline Zahlg. an öfftl. Bereich & 117 & 120 & 98 & 113 & 81 & 143 & 167 & 211 & 255 & 233 & 298 \\
\hline Bruttoausgaben & 238 & 256 & 225 & 259 & 248 & 392 & 453 & 499 & 511 & 569 & 662 \\
\hline I. Zahlungen v. gleicher Ebene & 0 & 0 & 0 & 0 & 0 & 0 & 0 & 0 & 0 & 0 & 0 \\
\hline Bereinigte Ausg. & 238 & 256 & 225 & 259 & 248 & 392 & 453 & 499 & 511 & 569 & 662 \\
\hline I. Zahlungen v. anderer Ebene & 23 & 24 & 39 & 32 & 32 & 31 & 35 & 35 & 77 & 9 & 56 \\
\hline Nettoausgaben & 215 & 232 & 186 & 227 & 216 & 362 & 419 & 465 & 436 & 521 & 607 \\
\hline Unmittelbare Ein & 12 & 9 & 11 & 13 & 9 & 21 & 24 & 25 & 30 & 30 & 28 \\
\hline Einn.aus wirtsch.Tätigkeit & & & & & & 5 & 6 & 5 & 7 & $\overline{6}$ & 4 \\
\hline Gebühren, Entgelte & 4 & 4 & 5 & 5 & 1 & 7 & 8 & 9 & 10 & 9 & 11 \\
\hline uschußbedarf & 203 & 223 & 175 & 214 & 207 & 341 & 395 & 440 & 406 & 491 & 579 \\
\hline
\end{tabular}

5.2. Gesamte Nettosportausgaben - nominal, getrennt nach Haushaitsabschnitten

\begin{tabular}{|l|r|r|r|r|r|r|r|r|r|r|r|}
\hline Jahr & 1965 & 1966 & 1967 & 1968 & 1969 & 1970 & 1971 & 1972 & 1973 & 1974 & 1975 \\
\hline Förderung des Sports & & & & & & 142 & 167 & 201 & 193 & 224 & 270 \\
\hline Sportstätten & & & & & & 187 & 204 & 196 & 164 & 209 & 268 \\
\hline Förd. d. Sports und Sportstätten & 215 & 232 & 186 & 227 & 216 & 329 & 371 & 397 & 357 & 433 & 538 \\
\hline Badeanstalten & & & & & & 33 & 48 & 68 & 79 & 87 & 69 \\
\hline Nettosportausgaben insgesamt & 215 & 232 & 186 & 227 & 216 & 362 & 419 & 465 & 436 & 521 & 607 \\
\hline
\end{tabular}

5.3. Gesamte Nettosportausgaben - nominal und real, Basisjahr 1985

\begin{tabular}{|l|r|r|r|r|r|r|r|r|r|r|r|}
\hline Jahr & 1965 & 1966 & 1967 & 1968 & 1969 & 1970 & 1971 & 1972 & 1973 & 1974 & 1975 \\
\hline nominal & 215 & 232 & 186 & 227 & 216 & 362 & 419 & 465 & 436 & 521 & 607 \\
\hline real & 471 & 492 & 388 & 468 & 436 & 708 & 780 & 822 & 722 & 808 & 887 \\
\hline
\end{tabular}


nominal, getrennt nach Ausgabe- und Einnahmearten

\begin{tabular}{|r|r|r|r|r|r|r|r|r|r|r|r|r|r|r|r|}
\hline 1976 & 1977 & 1978 & 1979 & 1980 & 1981 & 1982 & 1983 & 1984 & 1985 & 1986 & 1987 & 1988 & 1989 & 1990 & 1991 \\
\hline 347 & 374 & 467 & 482 & 500 & 503 & 495 & 505 & 529 & 536 & 546 & 599 & 601 & 633 & 681 & 822 \\
\hline 358 & 209 & 216 & 255 & 331 & 293 & 303 & 254 & 237 & 210 & 193 & 218 & 215 & 200 & 206 & 216 \\
\hline 706 & 584 & 683 & 738 & 831 & 796 & 797 & 761 & 766 & 747 & 739 & 817 & 816 & 833 & 885 & 1.038 \\
\hline 1 & 0 & 0 & 0 & 0 & 0 & 0 & 1 & 0 & 0 & 0 & 0 & 0 & 1 & 0 & 0 \\
\hline 705 & 584 & 683 & 738 & 831 & 796 & 797 & 760 & 766 & 747 & 739 & 817 & 815 & 833 & 885 & 1.038 \\
\hline 58 & 46 & 51 & 47 & 39 & 43 & 45 & 47 & 57 & 52 & 46 & 45 & 53 & 50 & 60 & 80 \\
\hline 647 & 535 & 632 & 691 & 792 & 753 & 752 & 713 & 709 & 695 & 693 & 772 & 762 & 782 & 825 & 957 \\
\hline 34 & 31 & 30 & 33 & 31 & 38 & 41 & 45 & 44 & 37 & 36 & 47 & 49 & 44 & 48 & 76 \\
\hline 4 & 3 & 3 & 4 & 4 & 4 & 5 & 6 & 0 & 0 & 0 & 0 & 0 & 0 & 0 & 0 \\
\hline 12 & 13 & 11 & 12 & 13 & 14 & 16 & 15 & 16 & 14 & 15 & 16 & 18 & 19 & 18 & 34 \\
\hline 613 & 504 & 602 & 658 & 761 & 715 & 711 & 668 & 665 & 658 & 657 & 725 & 713 & 738 & 777 & 881 \\
\hline
\end{tabular}

\begin{tabular}{|r|r|r|r|r|r|r|r|r|r|r|r|r|r|r|r|}
\hline 1976 & 1977 & 1978 & 1979 & 1980 & 1981 & 1982 & 1983 & 1984 & 1985 & 1986 & 1987 & 1988 & 1989 & 1990 & 1991 \\
\hline 275 & 207 & 187 & 196 & 208 & 208 & 203 & 202 & 213 & 227 & 234 & 259 & 253 & 268 & 285 & 309 \\
\hline 312 & 272 & 379 & 401 & 506 & 475 & 484 & 442 & 407 & 398 & 391 & 454 & 447 & 448 & 470 & 546 \\
\hline 587 & 479 & 566 & 597 & 714 & 683 & 687 & 644 & 620 & 625 & 625 & 713 & 700 & 716 & 755 & 855 \\
\hline 60 & 56 & 66 & 94 & 78 & 70 & 65 & 69 & 89 & 70 & 68 & 59 & 62 & 66 & 70 & 102 \\
\hline 647 & 535 & 632 & 691 & 792 & 753 & 752 & 713 & 709 & 695 & 693 & 772 & 762 & 782 & 825 & 957 \\
\hline
\end{tabular}

\begin{tabular}{|r|r|r|r|r|r|r|r|r|r|r|r|r|r|r|r|}
\hline 1976 & 1977 & 1978 & 1979 & 1980 & 1981 & 1982 & 1983 & 1984 & 1985 & 1986 & 1987 & 1988 & 1989 & 1990 & 1991 \\
\hline 647 & 535 & 632 & 691 & 792 & 753 & 752 & 713 & 709 & 695 & 693 & 772 & 762 & 782 & 825 & 957 \\
\hline 905 & 724 & 834 & 878 & 957 & 855 & 811 & 744 & 723 & 695 & 694 & 773 & 754 & 753 & 773 & 866 \\
\hline
\end{tabular}


5.4. Gesamte Nettosportausgaben - nominal, getrennt nach Bundesländern

\begin{tabular}{|l|r|r|r|r|r|r|r|r|r|r|r|}
\hline Jahr & 1965 & 1966 & 1967 & 1968 & 1969 & 1970 & 1971 & 1972 & 1973 & 1974 & 1975 \\
\hline Schleswig-Holstein & 4 & 4 & 4 & 7 & 19 & 15 & 17 & 12 & 9 & 11 & 8 \\
\hline Niedersachsen & 24 & 27 & 21 & 27 & 25 & 29 & 34 & 35 & 39 & 44 & 43 \\
\hline Nordrhein-Westfalen & 46 & 47 & 27 & 31 & 5 & 56 & 77 & 82 & 83 & 87 & 123 \\
\hline Hessen & 22 & 26 & 23 & 21 & 19 & 26 & 29 & 29 & 26 & 28 & 34 \\
\hline Rheinland-Pfalz & 12 & 9 & 8 & 9 & 10 & 18 & 17 & 26 & 26 & 30 & 25 \\
\hline Baden-Württemberg & 52 & 60 & 48 & 57 & 55 & 59 & 77 & 118 & 119 & 110 & 125 \\
\hline Bayern & 12 & 12 & 11 & 28 & 39 & 71 & 66 & 48 & -8 & 44 & 61 \\
\hline Saarland & 2 & 3 & 2 & 2 & 1 & 1 & 2 & 2 & 2 & 3 & 21 \\
\hline Hamburg & 12 & 13 & 12 & 13 & 11 & 19 & 17 & 23 & 20 & 33 & 30 \\
\hline Bremen & 9 & 9 & 9 & 10 & 13 & 16 & 18 & 16 & 22 & 35 & 51 \\
\hline Berlin & 21 & 21 & 22 & 24 & 18 & 50 & 64 & 70 & 98 & 95 & 84 \\
\hline Nettosportausgaben insg. & 215 & 232 & 186 & 227 & 216 & 362 & 419 & 465 & 436 & 521 & 607 \\
\hline
\end{tabular}

5.5. Gesamte Nettosportausgaben in DM pro Kopf - nominal, getrennt nach Bundesländern

\begin{tabular}{|l|r|r|r|r|r|r|r|r|r|r|r|}
\hline Jahr & 1965 & 1966 & 1967 & 1968 & 1969 & 1970 & 1971 & 1972 & 1973 & 1974 & 1975 \\
\hline Schleswig-Holstein & 1,65 & 1,63 & 1,61 & 2,78 & 7,47 & 6,01 & 6,72 & 4,7 & 3,5 & 4,26 & 3,1 \\
\hline Niedersachsen & 3,48 & 3,88 & 3,01 & 3,85 & 3,54 & 4,09 & 4,75 & 4,86 & 5,39 & 6,06 & 5,93 \\
\hline Nordrhein-Westfalen & 2,76 & 2,8 & 1,6 & 1,84 & 0,29 & 3,31 & 4,51 & 4,78 & 4,82 & 5,05 & 7,16 \\
\hline Hessen & 4,28 & 4,99 & 4,38 & 3,97 & 3,53 & 4,83 & 5,31 & 5,26 & 4,68 & 5,02 & 6,11 \\
\hline Rheinland-Pfalz & 3,36 & 2,5 & 2,21 & 2,48 & 2,73 & 4,94 & 4,63 & 7,06 & 7,03 & 8,12 & 6,8 \\
\hline Baden-Württemberg & 6,22 & 7,05 & 5,62 & 6,6 & 6,24 & 6,63 & 8,54 & 12,9 & 12,9 & 11,9 & 13,6 \\
\hline Bayern & 1,19 & 1,18 & 1,07 & 2,71 & 3,72 & 6,78 & 6,21 & 4,47 & $-0,74$ & 4,05 & 5,63 \\
\hline Saarland & 1,78 & 2,65 & 1,77 & 1,77 & 0,89 & 0,89 & 0,18 & 1,78 & 1,79 & 2,71 & 19,1 \\
\hline Hamburg & 6,46 & 7,02 & 6,52 & 7,12 & 6,04 & 10,6 & 9,5 & 13 & 11,4 & 18,9 & 17,4 \\
\hline Bremen & 12,2 & 12,1 & 12 & 13,3 & 17,2 & 22,1 & 24,4 & 21,7 & 30,1 & 48,1 & 70,7 \\
\hline Berlin & 9,54 & 9,58 & 10,1 & 11,2 & 8,42 & 23,6 & 30,5 & 33,8 & 47,7 & 46,7 & 41,9 \\
\hline
\end{tabular}

\section{Quellen:}

Statistisches Bundesamt, Finanzen und Steuern.

1965 bis 1969: Fachserie L, Reihe 5, Sonderbeiträge zur Finanzstatistik, Aufwendungen von Bund, Ländern und Gemeinden (GV) für Gesundheitspflege und Sport.

1970 bis 1973: Fachserie L, Reihe 5, Sonderbeiträge zur Finanzstatistik, Ausgaben der öffentlichen Haushalte furr Gesundheit, Sport und Erholung.

1974 bis 1979: Fachserie 14, Reihe 3.6, Rechnungsergebnisse der öffentlichen Haushalte für Gesundheit, Sport und Erholung.

Ab 1980: Fachserie 14, Reihe 3.5, Rechnungsergebnissse der öffentlichen Haushalte für soziale Sicherung und für Gesundheit, Sport und Erholung.

Statistisches Bundesamt, Statistische Jahrbücher. 


\begin{tabular}{|r|r|r|r|r|r|r|r|r|r|r|r|r|r|r|r|}
\hline 1976 & 1977 & 1978 & 1979 & 1980 & 1981 & 1982 & 1983 & 1984 & 1985 & 1986 & 1987 & 1988 & 1989 & 1990 & 1991 \\
\hline 49 & 11 & 12 & 14 & 16 & 16 & 14 & 14 & 15 & 13 & 12 & 12 & 14 & 17 & 16 & 14 \\
\hline 69 & 26 & 35 & 26 & 41 & 45 & 44 & 38 & 27 & 36 & 41 & 43 & 44 & 34 & 41 & 35 \\
\hline 107 & 86 & 97 & 119 & 168 & 117 & 73 & 65 & 75 & 77 & 71 & 73 & 64 & 77 & 87 & 84 \\
\hline 32 & 20 & 18 & 20 & 16 & 19 & 24 & 21 & 18 & 15 & 14 & 16 & 19 & 16 & 13 & 12 \\
\hline 34 & 35 & 38 & 43 & 51 & 57 & 68 & 54 & 54 & 54 & 54 & 54 & 52 & 50 & 62 & 65 \\
\hline 139 & 142 & 151 & 161 & 198 & 181 & 188 & 176 & 163 & 166 & 158 & 170 & 169 & 163 & 163 & 182 \\
\hline 51 & 51 & 58 & 102 & 109 & 111 & 112 & 103 & 102 & 82 & 109 & 131 & 129 & 139 & 147 & 139 \\
\hline 2 & 2 & 2 & 2 & 1 & 3 & 1 & 0 & 3 & 2 & 3 & 3 & 2 & 2 & 2 & 1 \\
\hline 42 & 33 & 32 & 29 & 30 & 40 & 48 & 37 & 39 & 38 & 35 & 34 & 34 & 32 & 34 & 44 \\
\hline 38 & 38 & 44 & 38 & 46 & 39 & 38 & 37 & 32 & 30 & 27 & 38 & 42 & 37 & 34 & 46 \\
\hline 82 & 92 & 144 & 137 & 118 & 124 & 143 & 165 & 183 & 184 & 168 & 200 & 190 & 217 & 224 & 333 \\
\hline 647 & 535 & 632 & 691 & 792 & 753 & 752 & 713 & 709 & 695 & 693 & 772 & 762 & 782 & 825 & 957 \\
\hline
\end{tabular}

\begin{tabular}{|r|r|r|r|r|r|r|r|r|r|r|r|r|r|r|r|}
\hline 1976 & 1977 & 1978 & 1979 & 1980 & 1981 & 1982 & 1983 & 1984 & 1985 & 1986 & 1987 & 1988 & 1989 & 1990 & 1991 \\
\hline 19 & 4,25 & 4,63 & 5,39 & 6,14 & 6,12 & 5,34 & 5,35 & 5,74 & 4,97 & 4,59 & 4,7 & 5,47 & 6,6 & 6,12 & 5,31 \\
\hline 9,54 & 3,6 & 4,84 & 3,6 & 5,66 & 6,2 & 6,06 & 5,24 & 3,73 & 5 & 5,7 & 6 & 6,14 & 4,71 & 5,59 & 4,71 \\
\hline 6,26 & 5,04 & 5,7 & 7 & 9,86 & 6,86 & 4,27 & 3,85 & 4,47 & 4,61 & 4,26 & 4,37 & 3,81 & 4,54 & 5,05 & 4,82 \\
\hline 5,77 & 3,61 & 3,25 & 3,6 & 2,86 & 3,39 & 4,28 & 3,76 & 3,24 & 2,71 & 2,53 & 2,9 & 3,43 & 2,86 & 2,27 & 2,07 \\
\hline 9,3 & 9,6 & 10,5 & 11,8 & 14 & 15,7 & 18,7 & 14,9 & 14,9 & 14,9 & 15 & 14,9 & 14,3 & 13,6 & 16,6 & 17,2 \\
\hline 15,2 & 15,6 & 16,5 & 17,6 & 21,4 & 19,5 & 20,3 & 19 & 17,6 & 17,9 & 17 & 18,3 & 18 & 17,1 & 16,8 & 18,4 \\
\hline 4,72 & 4,72 & 5,36 & 9,4 & 10 & 10,1 & 10,2 & 9,39 & 9,3 & 7,48 & 9,91 & 12 & 11,7 & 12,5 & 13 & 12,1 \\
\hline 1,83 & 1,84 & 1,86 & 1,87 & 0,94 & 2,82 & 0,94 & 0 & 2,85 & 1,91 & 2,87 & 2,84 & 1,9 & 1,89 & 1,87 & 0,93 \\
\hline 24,6 & 19,5 & 19,1 & 17,5 & 18,2 & 24,4 & 29,4 & 22,9 & 24,4 & 24 & 22,2 & 21,3 & 21,3 & 19,9 & 20,7 & 26,5 \\
\hline 53,2 & 53,7 & 62,8 & 54,6 & 66,2 & 56,3 & 55,2 & 54,3 & 47,7 & 45,2 & 41,1 & 57,6 & 63,6 & 55,5 & 50,1 & 67,3 \\
\hline 41,7 & 47,5 & 75,1 & 71,9 & 62,1 & 65,5 & 76,1 & 88,7 & 98,9 & 99,3 & 89,9 & 99,3 & 92,8 & 102 & 104 & 154 \\
\hline
\end{tabular}

\section{Erlăuterungen:}

Expliziter Ausweis der Badeanstalten auf Länderebene erst ab 1970.

Unterscheidung zwischen Förderung des Sports und Sportstätten auf Länderebene ab 1970, in einige Bundesländern noch später.

Zur Ermittlung der Pro-Kopf-Ausgaben wurden die durchschnittlichen Bevölkerungszahlen des Statistischen Bundesamtes herangezogen.

Leerzellen: keine Angaben möglich, da kein Ausweis. 


\section{Anhang 6: Die Sportausgaben des Bundes von 1965 bis 1991 in MIO DM}

6.1. Gesamte Sportausgaben (Förderung des Sports und Sportstätten) - nominal, getrennt

\begin{tabular}{|l|r|r|r|r|r|r|r|r|r|r|r|}
\hline Jahr & 1965 & 1966 & 1967 & 1968 & 1969 & 1970 & 1971 & 1972 & 1973 & 1974 & 1975 \\
\hline Unmittelbare Ausgaben & 4 & 6 & 9 & 20 & 31 & 104 & 375 & 563 & 105 & 27 & 32 \\
\hline Zahlg. an öffentlichen Bereich & 27 & 35 & 48 & 40 & 36 & 37 & 47 & 43 & 57 & 30 & 35 \\
\hline Bruttoausgaben & 31 & 41 & 57 & 61 & 67 & 141 & 422 & 606 & 163 & 57 & 67 \\
\hline /. Zahlg. v. gleicher Ebene & 0 & 0 & 0 & 0 & 0 & 0 & 0 & 0 & 0 & 0 & 0 \\
\hline Bereinigte Ausg. & 31 & 41 & 57 & 61 & 67 & 141 & 422 & 606 & 163 & 57 & 67 \\
\hline /. Zahlg. v. anderer Ebene & 0 & 0 & 0 & 0 & 0 & 0 & 0 & 0 & 0 & 0 & 0 \\
\hline Nettoausgaben & 31 & 41 & 57 & 61 & 67 & 141 & 422 & 606 & 163 & 57 & 67 \\
\hline Unmittelbare Einnahmen & 0 & 0 & 0 & 3 & 3 & 0 & 0 & 9 & 24 & 10 & 5 \\
\hline dar.: |lfd. Zuschüsse & 0 & 0 & 0 & 0 & 0 & 0 & 0 & 9 & 24 & 10 & 0 \\
\cline { 2 - 10 } & 0 & 0 & 0 & 3 & 3 & 0 & 0 & 0 & 0 & 0 & 5 \\
\hline Zuschubinnahmen & 0 & 41 & 57 & 57 & 63 & 141 & 422 & 597 & 139 & 47 & 62 \\
\hline
\end{tabular}

6.2. Gesamte Nettosportausgaben - nominal, getrennt nach Haushaltsabschnitten

\begin{tabular}{|l|r|r|r|r|r|r|r|r|r|r|r|}
\hline Jahr & 1965 & 1966 & 1967 & 1968 & 1969 & 1970 & 1971 & 1972 & 1973 & 1974 & 1975 \\
\hline Förderung des Sports & & & & & & 21 & 20 & 100 & 24 & 27 & 32 \\
\hline Sportstätten & & & & & & 120 & 402 & 506 & 139 & 30 & 35 \\
\hline Förd. des Sports u. Sportst. & 31 & 41 & 57 & 61 & 67 & 141 & 422 & 606 & 163 & 57 & 67 \\
\hline Badeanstalten & & & & & & 0 & 0 & 0 & 0 & 0 & 0 \\
\hline Nettoausgaben insgesamt & 31 & 41 & 57 & 61 & 67 & 141 & 422 & 606 & 163 & 57 & 67 \\
\hline
\end{tabular}

\subsection{Gesamte Nettosportausgaben - nominal und real, Basisjahr 1985}

\begin{tabular}{|l|r|r|r|r|r|r|r|r|r|r|r|}
\hline Jahr & 1965 & 1966 & 1967 & 1968 & 1969 & 1970 & 1971 & 1972 & 1973 & 1974 & 1975 \\
\hline nominal & 31 & 41 & 57 & 61 & 67 & 141 & 422 & 606 & 163 & 57 & 67 \\
\hline real & 68 & 87 & 119 & 126 & 135 & 276 & 786 & 1.071 & 270 & 88 & 98 \\
\hline
\end{tabular}

\section{Quellen:}

Statistisches Bundesamt, Finanzen und Steuern.

1965 bis 1969: Fachserie L, Reihe 5, Sonderbeiträge zur Finanzstatistik, Aufwendungen von Bund, Ländern und Gemeinden (GV) für Gesundheitspflege und Sport.

1970 bis 1973: Fachserie L, Reihe 5, Sonderbeiträge zur Finanzstatistik, Ausgaben der öffentlichen Haushalte für Gesundheit, Sport und Erholung.

1974 bis 1979: Fachserie 14, Reihe 3.6, Rechnungsergebnisse der öffentlichen Haushalte für Gesundheit, Sport und Erholung.

Ab 1980: Fachserie 14, Reihe 3.5, Rechnungsergebnissse der öffentlichen Haushalte für soziale Sichenung und für Gesundheit, Sport und Erholung. 
nach Ausgabe- und Einnahmearten

\begin{tabular}{|r|r|r|r|r|r|r|r|r|r|r|r|r|r|r|r|}
\hline 1976 & 1977 & 1978 & 1979 & 1980 & 1981 & 1982 & 1983 & 1984 & 1985 & 1986 & 1987 & 1988 & 1989 & 1990 & 1991 \\
\hline 39 & 39 & 44 & 44 & 49 & 47 & 49 & 49 & 55 & 53 & 54 & 59 & 72 & 69 & 78 & 176 \\
\hline 31 & 32 & 30 & 30 & 24 & 24 & 23 & 24 & 25 & 25 & 25 & 31 & 33 & 26 & 33 & 57 \\
\hline 70 & 71 & 74 & 74 & 73 & 71 & 72 & 73 & 80 & 78 & 79 & 90 & 105 & 95 & 111 & 233 \\
\hline 0 & 0 & 0 & 0 & 0 & 0 & 0 & 0 & 0 & 0 & 0 & 0 & 0 & 0 & 0 & 0 \\
\hline 70 & 71 & 74 & 74 & 73 & 71 & 72 & 73 & 80 & 78 & 79 & 90 & 105 & 95 & 111 & 233 \\
\hline 0 & 0 & 0 & 0 & 0 & 0 & 0 & 0 & 0 & 0 & 0 & 0 & 0 & 0 & 0 & 0 \\
\hline 70 & 71 & 74 & 74 & 73 & 71 & 72 & 73 & 80 & 78 & 79 & 90 & 105 & 95 & 111 & 233 \\
\hline 7 & 3 & 5 & 11 & 2 & 0 & 0 & 0 & 0 & 0 & 0 & 0 & 0 & 0 & 0 & 0 \\
\hline 3 & 0 & 0 & 11 & 2 & 0 & 0 & 0 & 0 & 0 & 0 & 0 & 0 & 0 & 0 & 0 \\
\hline 4 & 3 & 5 & 0 & 0 & 0 & 0 & 0 & 0 & 0 & 0 & 0 & 0 & 0 & 0 & 0 \\
\hline 63 & 68 & 69 & 63 & 71 & 71 & 72 & 73 & 80 & 78 & 79 & 90 & 105 & 95 & 111 & 233 \\
\hline
\end{tabular}

\begin{tabular}{|r|r|r|r|r|r|r|r|r|r|r|r|r|r|r|r|}
\hline 1976 & 1977 & 1978 & 1979 & 1980 & 1981 & 1982 & 1983 & 1984 & 1985 & 1986 & 1987 & 1988 & 1989 & 1990 & 1991 \\
\hline 39 & 39 & 44 & 44 & 49 & 47 & 49 & 49 & 55 & 53 & 54 & 59 & 72 & 69 & 78 & 176 \\
\hline 31 & 32 & 30 & 30 & 24 & 24 & 23 & 24 & 25 & 25 & 25 & 31 & 33 & 26 & 33 & 57 \\
\hline 70 & 71 & 74 & 74 & 73 & 71 & 72 & 73 & 80 & 78 & 79 & 90 & 105 & 95 & 111 & 233 \\
\hline 0 & 0 & 0 & 0 & 0 & 0 & 0 & 0 & 0 & 0 & 0 & 0 & 0 & 0 & 0 & 0 \\
\hline 70 & 71 & 74 & 74 & 73 & 71 & 72 & 73 & 80 & 78 & 79 & 90 & 105 & 95 & 111 & 233 \\
\hline
\end{tabular}

\begin{tabular}{|r|r|r|r|r|r|r|r|r|r|r|r|r|r|r|r|}
\hline 1976 & 1977 & 1978 & 1979 & 1980 & 1981 & 1982 & 1983 & 1984 & 1985 & 1986 & 1987 & 1988 & 1989 & 1990 & 1991 \\
\hline 70 & 71 & 74 & 74 & 73 & 71 & 72 & 73 & 80 & 78 & 79 & 90 & 105 & 95 & 111 & 233 \\
\hline 98 & 96 & 98 & 94 & 88 & 81 & 78 & 76 & 82 & 78 & 79 & 90 & 104 & 91 & 104 & 211 \\
\hline
\end{tabular}

\section{Erlăuterungen:}

Auf Bundesebene kein Ausweis von Ausgaben für Badeanstalten.

Unterscheidung zwischen Förderung des Sports und Sportstätten auf Bundesebene ab 1970.

Leerzellen: keine Angaben möglich, da kein Ausweis. 


\section{Anhang 7: Die Sportausgaben in der BRD von 1965 bis 1991 in MIO DM}

7.1. Gesamte Sportausgaben (Förderung des Sports, Sportstätten und Badeanstalten) von

\begin{tabular}{|c|c|c|c|c|c|c|c|c|c|c|c|c|}
\hline \multicolumn{2}{|c|}{ Jahr } & 1965 & 1966 & 1967 & 1968 & 1969 & 1970 & 1971 & 1972 & 1973 & 1974 & 1975 \\
\hline \multicolumn{2}{|c|}{ Unmittelbare Ausgaben } & 636 & 674 & 650 & 745 & 980 & 1.370 & 1.974 & 2.413 & 2.194 & 3.418 & 12 \\
\hline \multicolumn{2}{|c|}{ Zahlungen an öfftl. Bereich } & 164 & 173 & 167 & 176 & 146 & 222 & 269 & 320 & 381 & 380 & $\overline{447}$ \\
\hline \multicolumn{2}{|c|}{ Bruttoausgaben } & 800 & 847 & 817 & 922 & 1.125 & 1.591 & 2.244 & 2.733 & 2.574 & 3.799 & 4.059 \\
\hline \multicolumn{2}{|c|}{ I. Zahlungen v. gleicher Ebene } & 7 & 7 & 6 & 9 & 12 & 18 & 28 & 35 & 37 & 75 & 84 \\
\hline \multicolumn{2}{|c|}{ Bereinigte Ausgaben } & 793 & 842 & 811 & 912 & 1.114 & 1.573 & 2.216 & 2.697 & 2.537 & 3.723 & 3.975 \\
\hline \multicolumn{2}{|c|}{ I. Zahlungen $\mathrm{v}$. anderer Ebene } & 60 & 67 & 79 & 81 & 95 & 111 & 130 & 169 & 395 & 334 & 472 \\
\hline \multicolumn{2}{|c|}{ Nettoausgaben } & 733 & 775 & 733 & 831 & 1.019 & 1.463 & 2.087 & 2.528 & 2.145 & 3.350 & 3.504 \\
\hline \multicolumn{2}{|c|}{ Unmittelbare Einnahmen } & 92 & 108 & 140 & 148 & 173 & 230 & 258 & 320 & 426 & 498 & 546 \\
\hline & Einn. aus wirtsch.Tätigkeit & 2 & 3 & 3 & 4 & 6 & 17 & 20 & 21 & 27 & 40 & 44 \\
\hline & Gebühren, Entgelte & 57 & 69 & 92 & 86 & 105 & 119 & 146 & 164 & 197 & 272 & 352 \\
\hline & Sons & 0 & 0 & 0 & 7 & 3 & 0 & 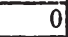 & 9 & 24 & 10 & 5 \\
\hline \multicolumn{2}{|c|}{ Zuschußbedarf } & 641 & 667 & 593 & 682 & 845 & 233 & 829 & 208 & 719 & 852 & 958 \\
\hline
\end{tabular}

7.2. Gesamte Nettosportausgaben aller Gebietskörperschaften (incl. Zweckverbände) -

\begin{tabular}{|l|r|r|r|r|r|r|r|r|r|r|r|}
\hline Jahr & 1965 & 1966 & 1967 & 1968 & 1969 & 1970 & 1971 & 1972 & 1973 & 1974 & 1975 \\
\hline Förderung des Sports & & & & & & 300 & 366 & 505 & 402 & 629 & 628 \\
\hline Sportstätten & & & & & & 659 & 1.068 & 1.226 & 780 & 1.229 & 1.344 \\
\hline Förd. des Sports und Sportstätten & 492 & 514 & 466 & 530 & 655 & 959 & 1.434 & 1.731 & 1.182 & 1.858 & 1.972 \\
\hline Badeanstalten & 241 & 261 & 267 & 300 & 364 & 506 & 653 & 797 & 962 & 1.501 & 1.537 \\
\hline Nettosportausgaben insgesamt & 733 & 775 & 733 & 831 & 1.019 & 1.463 & 2.087 & 2.528 & 2.145 & 3.359 & 3.509 \\
\hline
\end{tabular}

7.3. Gesamte Nettosportausgaben - nominal, getrennt nach Gebietskörperschaften

\begin{tabular}{|l|r|r|r|r|r|r|r|r|r|r|r|}
\hline Jahr & 1965 & 1966 & 1967 & 1968 & 1969 & 1970 & 1971 & 1972 & 1973 & 1974 & 1975 \\
\hline Bund & 31 & 41 & 57 & 61 & 67 & 141 & 422 & 606 & 163 & 57 & 67 \\
\hline Länder & 215 & 232 & 186 & 227 & 216 & 362 & 419 & 465 & 436 & 521 & 607 \\
\hline Gemeinden & 487 & 502 & 490 & 543 & 736 & 960 & 1.246 & 1.457 & 1.546 & 2.772 & 2.830 \\
\hline Zweckverbände & & & & & & & & & & 8 & 5 \\
\hline Nettoausgaben gesamt & 733 & 775 & 733 & 831 & 1.019 & 1.463 & 2.087 & 2.528 & 2.145 & 3.359 & 3.509 \\
\hline
\end{tabular}

\subsection{Gesamte Nettosportausgaben - nominal und real, Basisjahr 1985}

\begin{tabular}{|l|r|r|r|r|r|r|r|r|r|r|r|}
\hline Jahr & 1965 & 1966 & 1967 & 1968 & 1969 & 1970 & 1971 & 1972 & 1973 & 1974 & 1975 \\
\hline nominal & 733 & 775 & 733 & 831 & 1.019 & 1.463 & 2.087 & 2.528 & 2.145 & 3.359 & 3.509 \\
\hline real & 1.607 & 1.642 & 1.530 & 1.713 & 2.059 & 2.863 & 3.886 & 4.466 & 3.551 & 5.208 & 5.130 \\
\hline
\end{tabular}

\section{Quellen:}

Statistisches Bundesamt, Finanzen und Steuem.

1965 bis 1969: Fachserie L, Reihe 5, Sonderbeiträge zur Finanzstatistik, Aufwendungen von Bund, Ländern und Gemeinden (GV) für Gesundheitspflege und Sport.

1970 bis 1973: Fachserie L, Reihe 5, Sonderbeiträge zur Finanzstatistik, Ausgaben der öffentlichen Haushalte für Gesundheit, Sport und Erholung.

1974 bis 1979: Fachserie 14, Reihe 3.6, Rechnungsergebnisse der öffentlichen Haushalte für Gesundheit, Sport und Erholung.

Ab 1980: Fachserie 14, Reihe 3.5, Rechnungsergebnisse der öffentlichen Haushalte für soziale Sicherung und für Gesundheit, Sport und Erholung. 
Bund, Ländern und Gemeinden - nominal, getrennt nach Ausgabe- und Einnahmearten

\begin{tabular}{|c|c|c|c|c|c|c|c|c|c|c|c|c|c|c|c|}
\hline 976 & 77 & 78 & 979 & 1980 & 1981 & 1982 & 1983 & 1984 & 1985 & 1986 & 1987 & 1988 & 1989 & 1990 & 1991 \\
\hline 3.554 & 468 & 830 & 4.252 & 4.900 & 5.111 & 4.840 & 4.588 & 4.500 & 4.645 & 4.897 & 5.098 & 5.227 & 5.455 & 5.772 & $\overline{19}$ \\
\hline 499 & 347 & 347 & 398 & 490 & 465 & 454 & 386 & 368 & 337 & 324 & 347 & 343 & 328 & 342 & 371 \\
\hline 0.053 & 816 & 178 & 651 & 389 & 5.576 & .294 & 1.975 & 869 & 982 & 219 & .444 & 571 & \begin{tabular}{|l|l|}
783 \\
\end{tabular} & 113 & 572 \\
\hline 82 & 75 & 75 & 88 & 99 & 117 & 107 & $\overline{83}$ & 86 & 78 & 89 & 99 & 83 & 84 & 90 & 83 \\
\hline 3.970 & 3.740 & 4.103 & 4.563 & 5.290 & 5.460 & 5.187 & 4.892 & 4.782 & 4.905 & 5.130 & 5.344 & 5.485 & 5.700 & 5.024 & 6.489 \\
\hline 467 & 323 & 350 & 373 & 388 & 389 & 376 & 325 & 306 & 296 & 289 & 297 & 307 & 299 & 340 & 361 \\
\hline .503 & 3.415 & .752 & 4.189 & 4.900 & 5.071 & 4.812 & 4.568 & 4.477 & 4.608 & 4.842 & 5.048 & 5.178 & 5.401 & 5.684 & 6.125 \\
\hline 600 & 577 & 589 & 650 & 680 & 735 & 803 & 846 & 804 & 806 & 866 & 823 & 872 & 915 & 996 & 1.025 \\
\hline 52 & 56 & 59 & 64 & 71 & 79 & 85 & 98 & & & & & & & & \\
\hline 352 & 329 & 339 & 340 & 345 & 369 & 433 & 480 & 435 & 432 & 471 & 437 & 481 & 503 & 513 & 46 \\
\hline 7 & 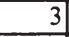 & 5 & 11 & 2 & 0 & 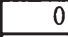 & & 0 & & & & 0 & & & \\
\hline 03 & 20 & 63 & 539 & 20 & 3 & 09 & 52 & 73 & 802 & 207 & 25 & 306 & 486 & 688 & 100 \\
\hline
\end{tabular}

nominal, getrennt nach Haushaltsabschnitten

\begin{tabular}{|r|r|r|r|r|r|r|r|r|r|r|r|r|r|r|r|}
\hline 1976 & 1977 & 1978 & 1979 & $\mathbf{1 9 8 0}$ & $\mathbf{1 9 8 1}$ & $\mathbf{1 9 8 2}$ & $\mathbf{1 9 8 3}$ & $\mathbf{1 9 8 4}$ & $\mathbf{1 9 8 5}$ & $\mathbf{1 9 8 6}$ & $\mathbf{1 9 8 7}$ & $\mathbf{1 9 8 8}$ & $\mathbf{1 9 8 9}$ & $\mathbf{1 9 9 0}$ & $\mathbf{1 9 9 1}$ \\
\hline 660 & 629 & 679 & $\mathbf{7 6 7}$ & $\mathbf{8 8 4}$ & $\mathbf{9 2 8}$ & $\mathbf{9 0 6}$ & $\mathbf{8 6 2}$ & $\mathbf{8 9 0}$ & $\mathbf{9 2 5}$ & 967 & 1.027 & 1.050 & 1.094 & 1.185 & 1.347 \\
\hline 1.357 & 1.372 & 1.632 & 1.832 & 2.240 & 2.294 & 2.11 & 1.936 & 1.816 & 1.905 & 2.048 & 2.188 & 2.254 & 2.332 & 2.398 & 2.559 \\
\hline 2.017 & 2.001 & 2.311 & 2.599 & 3.124 & 3.222 & 3.017 & 2.798 & 2.706 & 2.830 & 3.015 & 3.215 & 3.304 & 3.426 & 3.583 & 3.906 \\
\hline 1.497 & 1.422 & 1.442 & 1.598 & 1.782 & 1.855 & 1.806 & 1.779 & 1.779 & 1.785 & 1.835 & 1.839 & 1.884 & 1.986 & 2.112 & 2.230 \\
\hline 3.514 & 3.423 & 3.753 & 4.197 & 4.906 & 5.077 & 4.823 & 4.577 & 4.485 & 4.615 & 4.850 & 5.054 & 5.188 & 5.412 & 5.695 & 6.136 \\
\hline
\end{tabular}

\begin{tabular}{|r|r|r|r|r|r|r|r|r|r|r|r|r|r|r|r|}
\hline 1976 & 1977 & 1978 & 1979 & 1980 & 1981 & 1982 & 1983 & 1984 & 1985 & 1986 & 1987 & 1988 & 1989 & 1990 & 1991 \\
\hline 70 & 71 & 74 & 74 & 73 & 71 & 72 & 73 & 80 & 78 & 79 & 90 & 105 & 95 & 111 & 233 \\
\hline 647 & 535 & 632 & 691 & 792 & 753 & 752 & 713 & 709 & 695 & 693 & 772 & 762 & 782 & 825 & 957 \\
\hline 2.786 & 2.809 & 3.046 & 3.424 & 4.035 & 4.247 & 3.988 & 3.782 & 3.688 & 3.835 & 4.070 & 4.186 & 4.311 & 4.524 & 4.748 & 4.935 \\
\hline 10 & 8 & 2 & 8 & 7 & 7 & 13 & 9 & 7 & 8 & 9 & 7 & 8 & 11 & 10 & 9 \\
\hline 3.514 & 3.423 & 3.753 & 4.197 & 4.906 & 5.077 & 4.823 & 4.577 & 4.485 & 4.615 & 4.850 & 5.054 & 5.188 & 5.412 & 5.695 & 6.136 \\
\hline
\end{tabular}

\begin{tabular}{|l|l|l|l|l|l|l|l|l|l|l|l|l|l|l|l|}
\hline 1976 & 1977 & 1978 & 1979 & 1980 & 1981 & 1982 & 1983 & 1984 & 1985 & 1986 & 1987 & 1988 & 1989 & 1990 & 1991 \\
\hline 3 & 5 & 3.423 & 3.753 & 497 & 496 & 077 & 4 & &
\end{tabular}

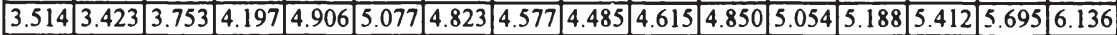

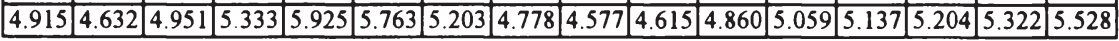

\section{Erläuterungen:}

Die Angaben zu den Badeanstalten beschränken sich - da ein Ausweis auf Länderebene erst ab 1970 erfolgt - für die Jahre 1965 bis 1969 auf die Kommunen. Die ausgewiesenen Nettosportausgaben unterschreiten die tatsächlichen Nettosportausgaben damit um die - allerdings geringe - Höhe der Bäderausgaben der Länder.

Unterscheidung zwischen Förderung des Sports und Sportstätten auf Bundes- und Länderebene erst ab 1970, in einigen Bundesländern noch später.

Leerzellen: keine Angaben möglich, da kein Ausweis. 
Anhang 8: Die Sportausgaben der Stadt Freiburg von 1973 bis 1991 in DM

\begin{tabular}{|c|c|c|c|c|c|}
\hline \multicolumn{6}{|c|}{ Laufende und einmalige Sportausgaben und ihre Bestimmungsfaktoren (Haushaltsansåtze) } \\
\hline Jahr & $\begin{array}{c}\text { Laufende } \\
\text { Sportausgaben }\end{array}$ & $\begin{array}{c}\text { Sport- } \\
\text { forderungs- } \\
\text { Investitionen }\end{array}$ & $\begin{array}{l}\text { Sportstätten- } \\
\text { Netto- } \\
\text { Investitionen }\end{array}$ & $\begin{array}{c}\text { Zweck- } \\
\text { Zuweisungen }\end{array}$ & $\begin{array}{l}\text { Nettogesamt- } \\
\text { steuern }\end{array}$ \\
\hline 1973 & 437.380 & 564.000 & 570.510 & 20.000 & 90.572 .350 \\
\hline 1974 & 497.840 & 300.000 & 908.440 & 20.000 & 111.393 .000 \\
\hline 1975 & 626.760 & 200.000 & 342.500 & 70.000 & 118.445 .510 \\
\hline 1976 & 821.320 & 410.000 & 400.000 & 80.000 & 114.140 .000 \\
\hline 1977 & 937.670 & 350.000 & 50.000 & 110.000 & 132.606 .500 \\
\hline 1978 & 1.227 .490 & 390.000 & 541.500 & 105.000 & 148.851 .500 \\
\hline 1979 & 1.355 .890 & 307.500 & 527.500 & 96.500 & 153.541 .500 \\
\hline 1980 & 1.657 .430 & 275.000 & 562.000 & 280.000 & 175.430 .000 \\
\hline 1981 & 1.353 .360 & 745.000 & -16.000 & 56.000 & 179.630 .000 \\
\hline 1982 & 1.806 .210 & 550.000 & 2.630 .000 & 0 & 174.735 .000 \\
\hline 1983 & 2.186 .100 & 450.000 & 495.000 & 60.000 & 174.190 .000 \\
\hline 1984 & 2.625 .520 & 840.750 & 1.094 .000 & 234.000 & 195.140 .000 \\
\hline 1985 & 2.492 .040 & 475.000 & 1.528 .000 & 150.000 & 188.940 .000 \\
\hline 1986 & 2.734 .300 & 350.000 & 438.800 & 100.000 & 200.573 .000 \\
\hline 1987 & 2.664 .640 & 350.000 & 621.000 & 0 & 211.039 .000 \\
\hline 1988 & 2.740 .300 & 350.000 & 885.000 & 250.000 & 219.078 .000 \\
\hline 1989 & 2.784 .960 & 350.000 & 1.625 .000 & 400.000 & 227.502 .000 \\
\hline 1990 & 2.680 .960 & 350.000 & 1.315 .000 & 800.000 & 225.010 .000 \\
\hline 1991 & 3.306 .590 & 350.000 & 2.992 .000 & 1.000 .000 & 256.720 .000 \\
\hline 1992 & 3.329 .310 & 350.000 & 1.142 .000 & 440.000 & 269.330 .000 \\
\hline 1993 & 3.815 .390 & 250.000 & 1.685 .000 & 0 & 296.070 .000 \\
\hline 1994 & 3.946 .460 & 250.000 & 775.000 & 0 & 309.110 .000 \\
\hline
\end{tabular}

Quelle: Stadt Freiburg, Haushaltspläne, 1973 bis 1994. 


\section{Anhang 9: Die Sportausgaben der Stadt Karlsruhe von 1972 bis 1991 in DM}

\begin{tabular}{|c|r|r|r|r|r|}
\hline \multicolumn{2}{|c|}{ Laufende und einmalige Sportausgaben und ihre Bestimmungsfaktoren (Haushaltsansătze) } \\
\hline Jahr & $\begin{array}{c}\text { Laufende } \\
\text { Sportausgaben }\end{array}$ & $\begin{array}{c}\text { Sport- } \\
\text { forderungs- } \\
\text { Investitionen }\end{array}$ & $\begin{array}{c}\text { Sportstätten- } \\
\text { Netto- } \\
\text { Investitionen }\end{array}$ & $\begin{array}{c}\text { Zweck- } \\
\text { Zuweisungen }\end{array}$ & $\begin{array}{c}\text { Nettogesamt- } \\
\text { steuern }\end{array}$ \\
\hline 1972 & 695.582 & 345.000 & 150.000 & 0 & 168.826 .400 \\
\hline 1973 & 725.700 & 572.000 & 423.000 & 0 & 187.178 .400 \\
\hline 1974 & 935.870 & 450.000 & 605.000 & 20.000 & 220.074 .300 \\
\hline 1975 & 1.065 .910 & 470.000 & 1.428 .889 & 161.111 & 232.766 .500 \\
\hline 1976 & 1.154 .880 & 500.000 & 5.298 .891 & 711.109 & 252.046 .500 \\
\hline 1977 & 1.240 .510 & 635.000 & 3.373 .334 & 1.476 .666 & 260.528 .300 \\
\hline 1978 & 1.282 .420 & 620.000 & 5.528 .891 & 971.109 & 277.428 .300 \\
\hline 1979 & 2.158 .600 & 1.902 .000 & 4.001 .778 & 1.013 .222 & 301.837 .500 \\
\hline 1980 & 2.217 .010 & 1.130 .000 & 9.261 .112 & 538.888 & 319.514 .500 \\
\hline 1981 & 2.741 .780 & 1.892 .500 & 12.560 .916 & 1.520 .554 & 336.549 .500 \\
\hline 1982 & 2.837 .980 & 1.820 .000 & 20.488 .230 & 3.273 .550 & 360.199 .500 \\
\hline 1983 & 3.503 .650 & 3.180 .000 & 19.314 .600 & 2.636 .770 & 317.682 .500 \\
\hline 1984 & 4.248 .650 & 1.886 .150 & 4.643 .559 & 997.021 & 333.082 .500 \\
\hline 1985 & 8.222 .990 & 2.413 .000 & 225.000 & & 0 \\
\hline 1986 & 8.657 .730 & 2.413 .000 & 222.000 & 345.198 .000 \\
\hline 1987 & 10.329 .530 & 1.955 .000 & 848.500 & & 358.998 .000 \\
\hline 1988 & 10.674 .670 & 1.685 .000 & 655.960 & 900.000 & 389.298 .000 \\
\hline 1989 & 10.212 .240 & 1.700 .000 & 737.000 & 175.000 & 400.498 .000 \\
\hline 1990 & 9.489 .130 & 1.700 .000 & 3.617 .000 & 3.300 .000 & 396.148 .000 \\
\hline 1991 & 10.891 .420 & 1.850 .000 & 9.452 .163 & 3.779 .037 & 440.528 .000 \\
\hline 1992 & 11.069 .780 & 1.700 .000 & 10.616 .785 & 3.790 .715 & 461.108 .000 \\
\hline 1993 & 11.918 .310 & 1.600 .000 & 15.582 .320 & 4.711 .680 & 505.138 .000 \\
\hline 1994 & 11.942 .410 & 1.600 .000 & 7.085 .932 & 417.068 & 529.038 .000 \\
\hline & & & & & \\
\hline
\end{tabular}

Quelle: Stadt Karlsruhe, Haushaltspläne, 1972 bis 1994.

Erläuterungen: Aufgrund der im Zusammenhang mit der Erstellung der Karlsnuher Europahalle und dem Ausbau des Wildparkstadions zum Teil im voraus, zum Teil auch erst im nachhinein in Ansatz gebrachten Zweckzuweisungen wurde dieser Haushaltsposten bereinigt und die Zuweisungen entsprechend den Ausgabeansätzen der jeweiligen Haushaltsjahre auf die verschiedenen Jahre der Bauzeit verteilt. 
Christian Hockenjos - 978-3-631-75159-6

Downloaded from PubFactory at 01/11/2019 07:36:54AM

via free access 


\section{Literaturverzeichnis}

Andel, G. E. van und Austin, D. R.: Physical Fitness and Mental Health: A Review of the Literature, in: Adapted Physical Activity Quarterly, 1/1984, S.207 - 220.

Andel, N. (1984): Zum Konzept der meritorischen Güter, in: Finanzarchiv, 42/1984/3, S. 630 - 648 .

----- (1990): Finanzwissenschaft, 2. Auflage, Tübingen 1990.

Anders, G., Strähl, E. u. Moor, A. (Hrsg.): Sport und Wirtschaft, 27. Magglinger Symposium, Magglingen 1988.

Arbeitsgemeinschaft Deutscher Sportämter, ADS (Hrsg.): Jahrbuch Gemeinde und Sport, 1994 und 1995, Münster 1994.

Arbeitsgemeinschaft Deutscher Sportämter, Landesgruppe Baden-Württemberg und Arbeitsgemeinschaft kommunaler Sportämter des Städtetages Baden-Württemberg (Hrsg.): Ergebnis einer Städtebefragung zum Thema Kommunale Sportförderung und Fragen der Sportentwicklung, bearbeitet von Stark, R. u. Stadler, K.H., unveröffentlicht, Karlsruhe 1994.

Arnold, V.: Theorie der Kollektivgüter, München 1992.

Atkinson, A. B. u. Stern, N. H.: Pigou, Taxation and Public Goods, in: Review of Economic Studies, 41/1974, S. 119 - 128.

Backhaus, K., Erichson, B., Plinke, W. u. Weiber, R.: Multivariate Analysemethoden Eine anwendungsorientierte Einführung, 7. Auflage, Berlin, Heidelberg, New York, Tokyo 1994.

Badischer Sportbund (Hrsg.): Richtlinien und Hinweise zur Gewährung von Zuschüssen für den Bau von Sportanlagen sowie für die Beschaffung von Sportgeräten, Freiburg 1992.

Becker, P. (Hrsg.) (1982): Sport und Sozialisation, Reinbek 1982.

----- (1989): Jugendsozialarbeit und Sport. Zur Tauglichkeit des Sports als sozialpolitisches Entsorgungs- und Steuerungsinstrument, in: Klein, M. (Hrsg.): Sport und soziale Probleme, Reinbek 1989, S. 172 - 192.

Berglas, E.: On the Theory of Clubs, in: The American Economic Review, 66/1976/2, S. $116-121$. 
Beuker, F.: Wer Sport treibt, lebt gesünder, Erkenntnisse präventiver Sportmedizin, in: Franke, E. (Hrsg.): Sport und Gesundheit, Reinbek bei Hamburg, 1986, S. 41 52.

Blank, E. (1976): Kommunaler Sportstättenbau bei unzureichender Finanzausstattung, in: Der Städtetag, 6/1976, S. 313.

---- (1983): Sportförderung der Gemeinden, in: Gieseler, K. (Hrsg.) u.a.: Der Sport in der Bundesrepublik Deutschland, Schriftenreihe Verbände in der Bundesrepublik, Band 6, Wiesbaden 1983, S. 177 - 183.

Bonus, H.: Öffentliche Güter: Verführung und Gefangenendilemma, in: List Forum, 10/1979/80/2, S. $69-102$.

Böning, D. (Hrsg.) u.a.: Sport, Rettung oder Risiko für die Gesundheit? 31. Deutscher Sportärztekongress in Hannover 1988, Köln 1988.

Bowen, H. R.: The interpretation of voting in the allocation of economic ressources, in: Quarterly Journal of Economics, 58/1943, S. 27 - 48; wiederabgedruckt in: Arrow, K. J. u. Scitovsky, T. (Hrsg.): Readings in Welfare Economics, London 1969, S. $115-132$.

Brecht, A.: Internationaler Vergleich der öffentlichen Ausgaben, Leipzig, Berlin 1932.

Brümmerhoff, D.: Finanzwissenschaft, 5. Auflage, München, Wien 1990.

Brunner, D. u.a.: Physical activity at work and the incidence of myocardial infarction, angina pectoris and death due to ischaemic heart disease, in: Journal of chronicle Diseases, 2/1960, S. 428.

Buchanan, J. M.: An Economic Theory of Clubs, in: Economica, 32/1965/125, S. 1 14.

Bundesministerium der Finanzen (Hrsg.) (1984): Sport und Steuern, Bonn 1984.

---- (1988): Gutachten der unabhängigen Sachverständigenkommission zur Prüfung des Gemeinnützigkeits- und Spendenrechts, Schriftenreihe des BMF, Heft 40, Bonn 1988.

Bundesministerium des Innern (Hrsg.): Siebter Sportbericht der Bundesregierung, Bonn 1991.

Bundesministerium für Arbeit und Sozialordnung (Hrsg.): Euroatlas, Soziale Sicherheit im Vergleich, Bonn 1993.

CDU (Hrsg.) (1979): Sportförderung - eine öffentliche Aufgabe?, Bonn 1979.

---- (1993): Sportprogramm der CDU, Bonn 1993. 
Clarke, E. H.: Multipart pricing of public goods, in: Public Choice, 11/1971, S. 17 33.

Coase, R. H.: The Marginal Cost Controversy, in: Economica, 13/1946/51, S. 169 182.

Colm, G.: Volkswirtschaftliche Theorie der Staatsausgaben, Tübingen 1927.

Cornes, R. C. u. Sandler, T.: The Theory of Externalities, Public Goods and Club Goods, Cambridge 1986.

Davis, O. A., u. Whinston, A. B.: Piecemeal Policy in the Theory of Second Best, in: Review of Economic Studies, 34/1967, S. 323 - 331.

De Serpa, A. C. (1977): A Theory of Discriminatory Clubs, in: Scottish Journal of Political Economy, 24/1977, S. 33 - 41.

----- (1978): Congestion, Pollution and Impure Public Goods, in: Public Finance, 33/1978/1 - 2, S. 68 - 83.

Dellmann, H. J.: Sportförderung des Bundes, in: Gieseler, K. (Hrsg.) u.a.: Der Sport in der Bundesrepublik Deutschland, Schriftenreihe Verbände in der Bundesrepublik Deutschland, Band 6, Wiesbaden 1983, S. 109 - 150.

Deppe, L.: Das Verhältnis der kommunalen Ausgaben zur Größe, Struktur, Funktion und Finanzkraft städtischer Gemeinden, Diss., Münster 1966.

Deutsche Olympische Gesellschaft: (Hrsg.) (1959): Goldener Plan, Frankfurt a.M. 1959.

----- (1976) DOG-Richtlinien für die Schaffung von Erholungs-, Spiel- und Sportanlagen - neue III. Fassung 1976, Frankfurt a.M. 1976.

Deutscher Leichtathletikverband (Hrsg.) u.a.: Sport - Ernährung - Gesundheit, Edition Sport und Wissenschaft, Bremen 1989.

Deutscher Sportbund (Hrsg.) (1978): Sport als gesellschaftlicher Faktor. Schriftenreihe Berichte und Analysen, Heft 40, Frankfurt a.M. 1978.

----- (1982): Bundesländer und kommunale Gebietskörperschaften als Partner der Vereine und Verbände, Berlin 1982.

---- (1984): Orientierungshilfen für die Planung und Errichtung von Speziellen Anlagen für einzelne Sportarten, Ergänzung des Kapitels V der "Richtlinien für die Schaffung von Erholungs-, Spiel- und Sportanlagen", III. Fassung 1976, Frankfurt a.M. 1984.

---- (1984 a): 3. Memorandum zum Goldenen Plan, Frankfurt a.M. 1984. 
----- (1986): Die Zukunft des Sports. Materialien zum Kongreß "Menschen im Sport 2000", Schorndorf 1986.

---- (1990): Sport in der Bundesrepublik Deutschland, 16. Auflage, Frankfurt a.M. 1990.

---- (1990 a): Die Gründerjahre des Deutschen Sportbundes, 2 Bände, Schorndorf 1990.

---- (1991): Bestandserhebung 1991, Frankfurt a.M. 1991.

---- (1993): Bestandserhebung 1993, Frankfurt a.M. 1993.

---- (1993 a): Goldener Plan Ost, 2. Auflage, Frankfurt a.M. 1993.

Deutscher Städtetag (Hrsg.) (1958): Leitsätze für die kommunale Sportpflege, Köln 1958.

----- (1987): Empfehlungen zur kommunalen Sportförderung, Köln 1987.

Die Grünen im Landtag von Baden-Württemberg (Hrsg.): Für eine andere Spiel- und Bewegungskultur, Entwurf eine Grünen Programms, Stuttgart 1986.

Dietrich, K., Heinemann, K. u. Schubert, M.: Kommerzielle Sportanbieter, Schorndorf 1990.

Dietrich, K. u. Heinemann, K (Hrsg.): Der nichtsportliche Sport, Schorndorf 1989.

Digel, H. (Hrsg.) (1988): Sport im Verein und Verband, Schriftenreihe des Ausschusses deutscher Leibeserzieher, Schorndorf 1988.

(1989): Sport in der Risikogesellschaft. Zum Konflikt zwischen Sport und Umwelt, in: Klein, M. (Hrsg.): Sport und soziale Probleme, Reinbek bei Hamburg 1989, S. $71-120$.

Downs, A.: Ökonomische Theorie der Demokratie, Tübingen 1968.

Durbin, J.: Testing for Serial Correlation in Least-Squares Regression when Some of the Regressors Are Lagged Dependent Variables, in: Econometrica, 38/1970, S. $410-421$.

Duss, R.: Sportstätten 1990, in: Statistisches Jahrbuch deutscher Gemeinden, 77/1990, S. 228 - 267.

Endres, A.: Neuere Entwicklungen in der Theorie der öffentlichen Güter, in: Zeitschrift für die gesamte Staatswissenschaft, 137/1981/1, S. 134 - 149.

Erz, W.: Wieviel Sport veträgt die Natur?, in: GEO, 1985/7, S. 140 - 156. 
Fabricant, S.: The Trend of Government Activity in the United States since 1900, New York 1952.

Faulhaber, G. R.: Cross-Subsidization: Pricing in Public Enterprises, in: The American Economic Review, 65/1975/12, S. 966 - 977.

FDP: Liberale Leitsätze zur Sportpolitik, Bonn 1993.

Feldstein, M. S.: Equity and Efficiency in Public Pricing: The Optimal Two-Part Tariff, in: Quarterly Journal of Economics, 80/1972, S. 175 - 187.

Folkins, C. H. u. Sime, W. E.: Physical Fitness and Mental Health, in: American Psychologist, 36/1981, S. $373-389$.

Franke, E. (Hrsg.): Sport und Gesundheit, Reinbek 1986.

Freie Hansestadt Bremen (Hrsg.): Richtlinien für die Sportförderung in Bremen vom 10.06.1991.

Freistaat Bayern, Bayerisches Staatsministerium für Unterricht, Kultus, Wissenschaft und Kunst (Hrsg.): Bildung und Kulturpflege in Bayern 1991.

Freistaat Sachsen, Sächsisches Staatsministerium für Kultus (Hrsg.): Landessportplan des Freistaates Sachsen 1991/92, vorläufiger Entwurf.

Frey, B. S. u. Meißner, W.: Zwei Ansätze der politischen Ökonomie - Marxismus und ökonomische Theorie der Politik, Frankfurt a.M., 1974.

Frey, B. S. (1981): Theorie demokratischer Wirtschaftspolitik, München 1981. (1984): International Political Economics, Oxford 1984.

Frey, G.: Sport und Gesundheit. Eine Herausforderung für die Trainingswissenschaft, in: Küpper, D. u. Kottmann, L. (Hrsg.): Sport und Gesundheit, Schorndorf 1991, S. $91-107$.

Frogner, E. u. Pilz, G. A.: Untersuchung zur Einstellung von jugendlichen Fußballspielern und -spielerinnen zu Regeln und Normen im Sport, in: Pilz, G. A. (Hrsg.) u.a.: Sport und Gewalt, Schriftenreihe des Bundesinstituts für Sportwissenschaft, Band 42, Schorndorf, 1982, S. 191 - 244.

Gabor, A.: A note on Block Tariffs, in: Review of Economic Studies, 23/1955-56, S. $32-41$.

Gärtner, M.: Sport in marktwirtschaftlichen Ordnungen: Was kann der Marktmechanismus im Sport leisten?, in: Anders, G., Strähl, E. u. Moor, A. (Hrsg.): Sport und Wirtschaft, 27. Magglinger Symposium, Magglingen 1988, S. 41 - 64. 
Gieseler, K. (Hrsg.) u.a.: Der Sport in der Bundesrepublik Deutschland, Schriftenreihe Verbände in der Bundesrepublik Deutschland, Band 6, Wiesbaden 1983.

Grossekettler, H.: Options- und Grenzkostenpreise für Kollektivgüter unterschiedlicher Art und Ordnung, in: Finanzarchiv, 43/1985, S. $211-252$.

Güldenpfennig, S.: Texte zur Sporttheorie und Sportpolitik, Köln 1980.

Gujarati, D. N.: Basis Econometrics, 2. Auflage, Singapur 1988.

Haass, H.: Sport und kommunale Entwicklungsplanung, Münster, Hamburg 1990.

Hagen, H.: Höchstrichterliche Rechtsprechung zum Problemkreis Nachbarschutz und Sportstätte, in: Kirchhof, P. (Hrsg.): Sport und Umwelt, Heidelberg 1992, S. 1 15.

Hansestadt Lübeck (Hrsg.): Durchführungsbestimmungen für die Jugendsportförderung in der Hansestadt Lübeck, Neufassung vom 03.11.1993.

Hanusch, H.: Theorie des öffentlichen Guts, Göttingen 1972.

Happe, B. (1979): Sportplanung als Teil kommunaler Planung, in: Der Städtetag, 3/1979, S. 134 - 137.

--- (1986): Sportanlagen schaffen oder schließen?, in: Der Städtetag, 1/1986, S. 15 18.

Hauser, W. u. Gläser, H.: Alpine Skiunfälle und Verletzungen, Schriftenreihe des deutschen Skiverbandes, München 1985.

Head, J. G.: On Merit Goods, in: Finanzarchiv, 25/1966, S. 1 - 29.

Heil, J.: Einführung in die Ökonometrie, München, Wien 1984.

Heinemann, K. (1974): Politische Ökonomie - Heute, Tübingen 1974.

---- (Hrsg.) (1982): Texte zur Ökonomie des Sports, Schorndorf 1984.

---- (1987): Betriebswirtschaftliche Grundlagen des Sportvereins, Schorndorf 1987.

---- (1987 b): Elemente einer Ökonomie des Sports, in: Jahrbuch für Sozialwissenschaft, 38/1987, S. 232 - 257.

---- (1988): Besonderheiten einer Ökonomie des Sports, in: Anders, G., Strähl, E. u. Moor, A. (Hrsg.): Sport und Wirtschaft, 27. Magglinger Symposium, Magglingen 1988, S. $65-78$.

--- (1990): Kommerzielle Sportanbieter, 1990. 
Heinilä, K.: Ethics of sport. University of Jyväskylä, department of sociology and planning for physical culture, research reports, Nr. 4, Jyväskylä 1974.

Heinze, R. G.: Verbändepolitik und Neokorporatismus. Zur politischen Soziologie organisierter Interessen, Opladen 1981.

Heiß, F.: Unfallverhütung und Nothilfe beim Sport, Schriftenreihe zur Praxis der Leibeserziehung und des Sports, Band 57, 2. Auflage, Schorndorf 1977.

Helpman, E.: On Optimal Community Formation, in: Economic Letters, 1979/I(3), S. $289-293$.

Hess, H.: Sportverletzungen, 3. Auflage, München 1984.

Hockenjos, Chr. (1992): Gemeindliche Sportausgaben 1991, in: Statistisches Jahrbuch deutscher Gemeinden, 79/1992, S. 278 - 305.

---- (1993): Sportfördermittel der Gemeinden - Die kommunalen Sportausgaben im Jahr 1991, in: Der Städtetag, 46/1993/10, S. 657 - 661.

Ilmarinen, M. u.a.: Sport and international Understanding, Berlin, Heidelberg, New York, Tokio 1984.

Jung, D. u. Ulmer, H.-V.: Bewegungsmangel - Gefahr für die Volksgesundheit?, in: Deutsches Ärzteblatt, 80/1983/37, S. 62 - 65.

Kaldor, N.: Welfare propositions of economics and interpersonal comparison of utility, in: The Economic Journal, 49/1939, S. 549 - 552, wiederabgedruckt in: Arrow, K. J. u. Scitovsky, T. (Hrsg.): Readings in Welfare Economics, London 1969, S. 387 - 389 .

Keller, Th.: Das Gesetz der überproportional zur Bevölkerung steigenden Ausgaben, in: Arndt, H. (Hrsg.): Sozialwissenschaftliche Untersuchungen, Albrecht, G. zum 80. Geburtstag, Berlin 1969, S. 345 - 361.

Kirchhof, P. (Hrsg.): Sport und Umwelt, Heidelberg 1992.

Klein, M.: Sport und soziale Probleme in: Sport und soziale Probleme, Reinbek 1989, S. $9-70$.

Kleine, W. u. Hautzinger, M. (Hrsg.):Sport und psychisches Wohlbefinden - Beiträge zum Lehren und Lernen im Gesundheitssport, Edition Sport und Wissenschaft, Aachen 1990.

Kleine, W. u. Fritsch, W. (Hrsg.): Sport und Geselligkeit - Beiträge zu einer Theorie von Geselligkeit im Sport, Edition Sport und Wissenschaft, Aachen 1990. 
Knight, Fr. H.: Some Fallacies in the Interpretation of Social Cost, in: Quarterly Journal of Economics, 38/1924/4, S. 582 - 606.

Knobloch, J.: Zur Frage der psychohygienischen Wirkungen des Sports, in: Bäumler, G., Hahn, E. u. Nitsch, J. R. (Hrsg.): Aktuelle Probleme der Sportpsychologie. Bericht über das 2. internationale Symposium am 04.08.1978 in München, Schorndorf 1979, S. 238 - 260.

Kühne, K.: Öffentliche Güter, meritorische Güter und Klubtheorie, in: Oettle, K. (Hrsg.): Offentliche Güter und öffentliche Unternehmen, Baden-Baden 1984, S. $117-136$.

Külp, B.: Wohlfahrtsökonomie I, Tübingen, Düsseldorf 1975.

Kunze, R.: Die Gemeindeordnung für Baden-Württemberg, 20. Auflage, Stuttgart 1989.

Küpper, D. u. Kottmann, L. (Hrsg.): Sport und Gesundheit, Schorndorf 1991.

Kutsch, Th. u. Wiswede, G. (Hrsg.): Sport und Gesellschaft: Die Kehrseite der Medaille, Hain 1981.

Land Baden-Württemberg, Ministerium für Kultus und Sport (1969) (Hrsg.): Richtlinien für die Verwendung staatlicher Mittel zur Förderung des Sports, Amtsblatt Kultus und Unterricht, Sondernr. 1/1969, Stuttgart 1969.

Land Baden-Württemberg, Ministerium für Kultus und Sport (Hrsg.): Landessportpläne 1975 bis 1992.

Land Brandenburg, Ministerium für Bildung, Jugend und Sport (Hrsg.): Haushaltspläne 1991 und 1992.

Land Hessen, Hessisches Ministerium des Innern und für Europaangelegenheiten (Hrsg.): Aufstellung der Gesamtaufwendungen des Landes Hessen für den Sport von 1987 bis 1992.

Land Mecklenburg-Vorpommern, Die Kultusministerin des Landes Mecklenburg-Vorpommern (Hrsg.): Sportförderung in Mecklenburg-Vorpommern in den Jahren 1991 und 1992, Zusammenstellung verschiedener Materialien.

Land Niedersachsen, Niedersächsisches Kultusministerium (Hrsg.): Ausarbeitung zur Sportförderung des Landes Niedersachsen vom Dezember 1991.

Land Nordrhein-Westfalen, Kultusministerium des Landes Nordrhein-Westfalen (Hrsg.): Sportvereine in Nordrhein-Westfalen, Schriftenreihe Sport in NordrheinWestfalen, Heft 2, Köln 1979.

---.- : Landessportpläne 1980 bis 1992. 
Land Nordrhein-Westfalen, Der Kultusminister NRW (Hrsg.): Sportstättenstatistik für das Land Nordrhein-Westfalen, Düsseldorf 1988.

Land Rheinland-Pfalz, Ministerium des Innern und für Sport (Hrsg.): Übersicht über die Leistungen des Landes Rheinland-Pfalz zur Förderung des Sports in den Haushaltsjahren 1989 bis 1992.

Land Thüringen, Thüringer Ministerium für Soziales und Gesundheit (Hrsg.): Landeshaushalte 1991 und 1992.

Landeshauptstadt München (Hrsg.) (1991): Richtlinien für die Gewährung von Zuwendungen zur Neuerrichtung, Erweiterung, Verbesserung und Großinstandsetzung von vereinseigenen Sportanlagen, Neufassung vom 13.03.1991.

-.--- (1986 a): Richtlinien für die Gewährung von Zuschüssen zum Unterhalt vereinseigener Sportanlagen, Neufassung gemäß Beschluß des Sportausschusses vom 15.04.1986.

(1986 b): Richtlinien für die Verleihung von Auszeichnungen für hervorragende sportliche Leistungen, Beschluß des Sportausschusses, Neufassung gemäß Beschluß des Sportausschusses vom 25.11.1986.

----- (1989): Richtlinien für die Förderung der Jugendarbeit in Sportvereinen vom 01.10.1989.

----- (1991): Richtlinien zur Bezuschussung Münchener Sportvereine aus Einnahmen der Bandenwerbung auf städtischen Bezirkssportanlagen und Sportplätzen vom 02.04.1991.

----- (1993): Richtlinien für die Gewährung von Zuschüssen zur Teilnahme an Meisterschaften und Pokalwettbewerben, Neufassung gemäß Beschluß des Sportausschusses vom 28.09.1993.

----- (1994): Richtlinien für die Gewährung von Zuschüssen zur Teilnahme an den Rundenspielen der Amateur-Bundesliga, Neufassung gemäß Beschluß des Sportausschusses vom 01.03.1994.

Landeshauptstadt Saarbrücken (Hrsg.): Richtlinien der städtischen Sportförderung vom 01.01.1992.

Landeshauptstadt Stuttgart (Hrsg.) (1989): Richtlinien für die finanzielle Sportförderung vom 01.05.1989, ergänzte Fassung vom 01.09.1993.

----- (1992): Überlegungen des Sportamts zur Haushaltskonsolidierung, 3. Entwurf vom 09.04.1992. 
---- (1993): Rundschreiben der Stadt Stuttgart an die Nutzer städtischer Sportstätten zur Erhebung eines Sachkostenbeitrags (SKB) für die Nutzung städtischer Sportstätten vom 14.07.1993.

---- (1994): Haushaltsplan 1993/94.

Landessportbund Nordrhein-Westfalen (Hrsg.): Investitionshilfeprogramm für Vereine, Richtlinien zur Förderung der Sportvereine im investiven Bereich im Rahmen der Vereinshilfe des LSB NW e.V., beschlossen vom Hauptausschuß des LSB NW am 12. Dezember 1991 in Duisburg.

Lang, G. E.: Der Ausbruch von Tumulten bei Sportveranstaltungen, in: Lüschen, G. u. Weis, K. (Hrsg.): Die Soziologie des Sports, Darmstadt 1976, S. 273 - 295.

Lehner, Fr.: Einführung in die Neue Politische Ökonomie, Königstein/Ts. 1981.

Lepelmeier, D. u. Theurl, E.: Zur Problematik meritorischer Güter, in: WiSt, 10/1981/12, S. 553 - 556.

Lewis, W. A.: The Two-Part Tariff, in: Economica, 8/1941/31, S. 249 - 270.

Lindahl, E.: Die Gerechtigkeit der Besteuerung - eine Analyse der Steuerprinzipien auf der Grundlage der Grenznutzentheorie, Lund 1919.

Littmann, K.: Ausgaben, öffentliche II, Die "Gesetze" ihrer langfristigen Entwicklung, in: Albers, W. (Hrsg.) u.a.: Handwörterbuch der Wirtschaftswissenschaft (HdWW), erster Band, Stuttgart, New York, Tübingen, Göttingen, Zürich 1977, S. 349 - 363.

Lorenz, K.: Das sogenannte Böse. Zur Naturgeschichte der Aggression, Wien 1963.

Löw, R.: Sportstätten in Baden-Württemberg, in: Statistisches Landesamt BadenWürttemberg, Monatszeitschrift Baden-Württemberg in Wort und Zahl, 41/1993/2, S. 60 - 67.

Margolis, J.: Welfare Criteria, Pricing and Decentralization of a Public Service, in: Quarterly Journal of Economics, 71/1957, S. 448 - 463.

Mathieu, Th. (1980): Kommune und Sport, in: Der Städtetag, 1980/1, S. 4 - 8.

---- (1980 a): Gemeinden und Sportvereine als Partner, in: Der Städtetag, 1980/5, S. 287 - 289.

---- (1982): Kommune und Sport - Sportentwicklung und Sportförderung heute, in: Der Städtetag, 1982/3, S. 195 - 200. 
Mattner, H. (1983): Gemeindliche Sportförderung, dargestellt anhand kommunaler Sportförderungs-Richtlinien, in: Städte- und Gemeindebund, 1983/4, S. 135 140.

-.-- (1984): Gemeindliche Sportstättenentwicklungsplanung - Notwendigkeit und Rahmenbedingung, in: Städte- und Gemeindebund, 1984/12, S. 617 - 623.

Mattner-Stellmann, H.: Gemeindliche Sportpolitik der 90er Jahre, Rahmenbedingungen und Einflußfaktoren, in: Städte- und Gemeindebund, 1987/4, S. 189 - 197.

Millward, R.: Public expenditure economics, London 1971.

Mitchell, Br. M.: Optimal Pricing of Local Telephone Service, in: The American Economic Review, 68/1978/4, S. 517 - 537.

Mombauer, P. M. u. Mattner-Stellmann, H.: Sport 2000 - 8500 Goldene Planungen?, in: Städte- und Gemeindebund, 1988/1, S. 3 - 15.

Morris, I. N.: Incidence and prediction of ischaemic heartdisease in London busmen, in: Lancet, 1966/2, S. 553 - 559.

Mrazek, J.: Psyche, Gesundheit und Sport. Zur subjektiven Wahrnehmung des Gesundheitsversprechens durch den Sport, in: Franke, E. (Hrsg.): Sport und Gesundheit, Reinbek 1986, S. 86 - 97.

Munduch, G. u. Nitschke, E.: Klubtheorie, in: WiSt, 17/1988/6, S. 318 - 321.

Musgrave, R. A. (1957): A Multiple Theory of Budget Determination, in: Finanzarchiv, 17/1956-57, S. $333-343$.

---- (1957 a): Principles of Budget Determination, in: Joint Economic Committee, Federal Expenditure Policy for Economic Growth an Stability, 1957, S. 108 - 115.

----- (1959): The Theory of Public Finance, New York, Toronto, London 1959.

---- (1966): Finanztheorie; deutsche Übersetzung der Theory of Public Finance, Tübingen 1966.

u. Musgrave, P. B. (1984): The Public Finance in Theory and Practice, 4. Auflage, New York, 1984, (5. Auflage, 1988).

-----, ----- u. Kullmer, L. (1987): Die öffentlichen Finanzen in Theorie und Praxis, deutsche Übersetzung von (1984), 4. Auflage, Tübingen 1987, (5. Auflage 1990).

NG, Y.-K. (1973): The Economic Theory of Clubs: Pareto Optimality Conditions, in: Economica, 40/1973/159, S. 291 - 298. 
---- (1974): The Economic Theory of Clubs: Optimal Tax/ Subsidy, in: Economica, 41/1974/163, S. 308 - 321.

----- u. Weisser, M.: Optimal Prizing with a Budget Constraint - The Case of the TwoPart Tariff, in: Review of Economic Studies, 41/1974/127, S. 337 - 345.

Niskanen, W. A. jr.: Bureaucracy and Representative Government, Chikago 1971.

Noeske, W.: Steuereinnahmen 1991, in: Statistisches Jahrbuch deutscher Gemeinden, 79/1992, S. 426 - 453.

Oakland, W. H.: Congestion, Public Goods and Welfare, in: Journal of Public Economics, 1/1972/3-4, S. $339-357$.

Oettle, K. (Hrsg.): Öffentliche Güter und öffentliche Unternehmungen, Baden-Baden 1984.

Oi, W. Y.: A Disneyland Dilemma: Two-part Tariffs For A Mickey Mouse Monopoly, in: Quarterly Journal of Economics, 85/1971/1, S. 77 - 96.

Olson, M. jr. (1965): The Logic of Collective Action: Public Goods and the Theory of Groups, Cambridge/Mass. 1965.

----- (1968): Die Logik kollektiven Handelns, Tübingen 1968.

-.--- u. Zeckhauser, R.: An Economic Theory of Alliances, in: Review of Economics and Statistics, 48/1966, S. 266 - 279.

Pelshenke, G. (1961): Aufstellung von Leitplänen für Erholungs-, Spiel- und Sportanlagen als Teilpläne der Flächennutzungspläne in Stadt- und Landkreisen. Protokolle der fünften Arbeitstagung "Übungsstättenbau", Saarbrücken 1961.

----- (1988): Sportförderung zwischen Mäzenatentum und Sponsorship, Trier, 1988

Pethig, R.: Verbände als Produzenten öffentlicher Güter, in: Wirtschaftspolitische Blätter, 33/1986/2, S. $145-154$.

Petersen, H.-G. (1988): Finanzwissenschaft II, Stuttgart, Berlin, Köln, Mainz 1988.

---- (1993): Finanzwissenschaft I, 3. Auflage, Stuttgart, Berlin, Köln 1993.

Paffenberger, R. S.: Körperliche Aktivität, Leistungsfähigkeit, koronare Herzkrankheit und Lebenserwartung, übersetzt aus dem Englischen von Rost, $R$. in: Deutsche Zeitschrift für Sportmedizin, 42/1991/2, S. 60 - 66.

Pigou, A. C.: The Economics of Welfare, London 1920.

Pilz, G. A. (1982) (Hrsg.) u.a.: Sport und körperliche Gewalt, Reinbek 1982. 
Pilz, G. A. u.a. (1982 a): Sport und Gewalt, Schriftenreihe des Bundesinstituts für Sportwissenschaft, Band 42, Schorndorf 1982.

Pommerehne, W. W. u. Frey, Br. S.: Ökonomische Theorie der Politik, Berlin, Heidelberg, New York 1979.

Renström, P. u. Peterson, L.: Verletzungen im Sport, deutsche Übersetzung, Köln-Lövenich 1981.

Roppel, U.: Ökonomische Theorie der Bürokratie, Freiburg 1979.

Rost, R. (1989): Können Abstriche am Sterbegeld den Tod unseres Gesundheitssystems verhindern?, in: Deutsche Zeitschrift für Sportmedizin, 40/1989/3, S. 79.

---- (1989 a): Schlußwort zur Diskussion, in: Deutsche Zeitschrift für Sportmedizin, 40/1989/8, S. 292 - 294.

Ruggles, N. D. (1949): The Welfare Basis of the Marginal Cost Pricing Principle, in: The Review of Economic Studies, 17/1949/50, S. 28 - 46.

---- (1950): Recent Developments in the Theory of Marginal Cost Pricing, in: The Review of Economic Studies, 17/1950/43, S. 107 - 126.

Samuelson, P. A. (1954): The Pure Theory of Public Expenditure, in: The Review of Economics and Statistics, 36/1954/11, S. 387 - 389.

---- (1955): A Diagrammatic Exposition of a Theory of Public Expenditure, in: The Review of Economics and Statistics, 37/1955/11, S. 350 - 356, übersetzt ins Deutsche von, Recktenwald, H. C. (Hrsg.): Eine Theorie der öffentlichen Ausgaben, in: Finanztheorie, Köln, Berlin 1970, S. 146 - 158.

(1972): Pure Theory of Public Expenditure and Taxation, in: Margolis, J. u. Guitton, H. (Hrsg.): Public Economics: An Analysis of Public Production and Consumption and their Relations to the Private Sector; Proceedings of a Conference held by the International Economic Association, London 1969, abgedruckt in: The Collected Scientific Papers of Paul A. Samuelson, Vol. III, Cambridge/Mass., London 1972, S. 492 - 517.

Sander, L.: Aufgaben und Einnahmen der Kommunen in der Bundesrepublik Deutschland, Diss., Münster 1986.

Sandler, T.: Club Optimality. Further Clarifications, in: Economic Letters, Amsterdam, 14/1984/1, S. $61-65$.

u. Tschirhart, J. T.: The Economic Theory of Clubs: An Evaluative Survey, in: Journal of Economic Literature, 18/1980/12, S. 1481 - 1521. 
Schmidt, K. (1979): Sportprogramme der politischen Parteien, Schriftenreihe zum deutschen Sporthandbuch, Band 1, Wiesbaden 1979.

---- (1983): Sportförderung in den Bundesländern, in: Gieseler, K. (Hrsg.) u.a.: Der Sport in der Bundesrepublik Deutschland, Schriftenreihe Verbände in der Bundesrepublik, Band 6, Wiesbaden 1983, S. 151 - 176.

Schmidt, K. (1970): Kollektivbedürfnisse und Staatstätigkeit, in: Theorie und Praxis des finanzpolitischen Interventionismus, Tübingen 1970, S. 3 - 27.

---- (1988): Mehr zur Meritorik, in: Zeitschrift für Wirtschafts- und Sozialwissenschaften, 108/1988, S. 282 - 403.

Schumpeter, J.: Kapitalismus, Sozialismus und Demokratie, 2. Auflage, Bern 1950.

Scitovsky, T.: A Note on Welfare Propositions in Economics, in: The Review of Economic Studies, 9/1941, S. 77 - 88; wiederabgedruckt in: Arrow, K. J. u. Scitovsky, T. (Hrsg.): Readings in Welfare Economics, London 1969, S. 390 - 401.

Sherman, R.: Club Subscriptions for Public Transport Passengers, in: Journal of Transport Economics and Policy, 1/1967, S. 237 - 242.

Smith, B. D.: Social Determinants of Violence in Hockey. A Review, in: Canadian Journal of Applied Sport Sciences, 4/1979, S. 76 - 82.

Sorenson, J. R., Tschirhart, J. T. u. Whinston, A. B.: A Theory of Pricing under Decreasing Costs, in: The American Economic Review, 68/1978/4, S. 614 - 624.

SPD (1968): Empfehlungen zur Sportförderung in Städten, Gemeinden und Landkreisen, Bonn 1968.

---- (1993): Politik, Das neue Sportprogramm der SPD: Lebensqualität und Lebensfreude, Bonn 1993.

Stadler, K.-H.: Der Großverein und seine Sportstätten - Förderung vereinseigener Sportstätten aus kommunaler Sicht, niedergeschriebene Fassung eines Referats beim Freiburger Kreis, Darmstadt 08.05.1993.

Stadt Aachen (Hrsg.): Richtlinien der Stadt Aachen für die Förderung des Sports im Stadtbezirk Aachen, Beschluß der Sitzung der Bezirksvertretung vom 09.12.1975, geänderte Fassung vom 23.05.1984.

Stadt Augsburg (Hrsg.): Richtlinien der Stadt Augsburg zur Förderung der Augsburger Sportvereine vom 19.11.1974, in der Fassung der Änderungsbeschlüsse des Stadtrates vom 12.11.1992. 
Stadt Bad Salzuflen (Hrsg.): Sportförderungsrichtlinien der Stadt Bad Salzuflen, Neufassung gemäß Beschluß des Hauptausschusses der Stadt Bad Salzuflen vom 28.11.1990.

Stadt Bochum (Hrsg.): Richtlinien zur Förderung von Sportverbänden und Sportvereinen vom 29.01.1976.

Stadt Braunschweig (Hrsg.): Ergebnis einer durch die Stadt Braunschweig durchgeführten Umfrage zur Gewährung von Unterhaltungszuschüssen an Sportvereine, Braunschweig 1992.

Stadt Bruchsal (Hrsg.): Richtlinien zur Förderung der Bruchsaler Vereine, Beschluß der Gemeinderatssitzung vom 15.10.1991.

Stadt Delbrück (Hrsg.): Sportförderungsrichtlinien der Stadt Delbrück, Neufassung gemäß Ratsbeschluß vom 29.03.1990.

Stadt Dortmund (Hrsg.): Sportförderung in Dortmund, Beiträge zur Stadtentwicklung, Nr. 3, 1976.

Stadt Dreieich (Hrsg.): Sport- und Vereinswegweiser, 2. Auflage, Dreieich 1991.

Stadt Esslingen (Hrsg.): Städteumfrage Sportförderung, Esslingen 1990.

Stadt Forchheim (Hrsg.): Sportförderungsrichtlinien der Stadt Forchheim, Beschluß Kulturausschuß vom 16.01.1986, TOP 2/ Stadtrat vom 30.01.1986, in der Fassung vom 18.05.1993.

Stadt Freiburg i.Br. (Hrsg.) (1976): Sportstättenleitplan der Stadt Freiburg i.Br., Heft 4, Freiburg 1976.

---- (1988): Sport-Förderungsrichtlinien der Stadt Freiburg i.Br., Beschluß des Gemeinderates vom 12.10.1988.

---- : Haushaltspläne 1973 bis 1994.

Stadt Fröndenberg (Hrsg.): Sportförderungs-Richtlinien der Stadt Fröndenberg, Beschluß des Rates der Stadt Fröndenberg vom 19.05.1976.

Stadt Ganderkesee (Hrsg.): Richtlinien für die außerschulische Sportförderung sowie sonstige Förderung investiver Vorhaben von Verbänden, Vereinen und Gruppen, Neufassung vom 16.12.1993.

Stadt Geislingen an der Steige (Hrsg.): Richtlinien über die Gewährung von Förderungsbeiträgen durch die Stadt an die örtlichen Turn- und Sportvereine in der Fassung des Gemeinderatsbeschlusses vom 30.08.1989, geändert am 25.09.1991. 
Stadt Heidelberg (Hrsg.): Richtlinien der Stadt Heidelberg für die Bewilligung von Zuschüssen zur Sportförderung im Rahmen des IX. Sportförderungsprogrammes 1992 - 1994 vom 19.12.1991.

Stadt Heilbronn (Hrsg.): Richtlinien über die finanzielle Sportförderung und Förderung der Hobby- und Freizeitvereine der Stadt Heilbronn, Neufassung gemäß Gemeinderatsbeschluß vom 10.12.1992.

Stadt Hof (Hrsg.): Sportförderrichtlinien, Hof 1994.

Stadt Homburg (Saar) (Hrsg.): Zuschußrichtlinien Sport, Beschluß des Rates der Stadt Homburg vom 24.05.1978.

Stadt Karlsruhe (Hrsg.) (1991): Sportförderungsrichtlinien der Stadt Karlsruhe vom 11.03.1981 in der Fassung des Gemeinderatsbeschlusses vom 20.03.1991.

----- (1992) Sportdezernat und Sport- und Bäderamt (Hrsg.): Untersuchung zur Sportentwicklung, Sportanbieter in Karlsruhe, bearbeitet von Stark, R. u. Stadler, K.H., Karlsruhe 1992.

----- : Haushaltspläne 1972 bis 1994.

Stadt Kehl (Hrsg.): Richtlinien zur Förderung der Vereinigungen vom 18.05.1983, geänderte und ergänzte Fassung vom 01.09.1992.

Stadt Koblenz (Hrsg.): Richtlinien über die Förderung von Sport in Koblenz in der Fassung vom 04.05.1993.

Stadt Köln (Hrsg.): Beihilfenordnung zur Förderung des Kölner Sports vom 15.03.1977 in der Fassung vom 29.05.1990.

Stadt Konstanz (Hrsg.): Richtlinien für die Sportförderung vom 15.09.1987.

Stadt Lahr (Hrsg.) (1993): Richtlinien über die Fördenung der sporttreibenden Vereine in der Stadt Lahr/Schwarzwald vom 12.01.1993.

---- (1993 a): Allgemeine Vertragsbedingungen für die Überlassung städtischer Turnund Sporthallen sowie Gymnastikräume und für sportliche Nutzungen der Mehrzweckhallen, Neufassung vom 12.01.1993.

----- (1993 b): Benutzungsordnung für die Turn- und Sporthallen, die Gymnastikräume und für sportliche Nutzungen von Mehrzweckhallen der Stadt Lahr/Schwarzwald, Neufassung vom 12.01.1993.

---- : Haushaltspläne 1973 bis 1994.

Stadt Langen (Hrsg.): Richtlinien der Stadt Langen zur Förderung des Sports, Neufassung zum 01.07.1992. 
Stadt Lehrte (Hrsg.): Richtlinien für die Sportförderung in der Stadt Lehrte, Sportförderungsrichtlinien vom 18.08.1993.

Stadt Leimen (Hrsg.): Sportförderrichtlinien der Stadt Leimen, 6. Vereinszuschußprogramm der Stadt Leimen für die Zeit von 1992 - 1994, 6. Auflage, Leimen 1992.

Stadt Lennestadt (Hrsg.): Sportförderungsrichtlinien der Stadt Lennestadt vom 27.02.1978 in der Fassung vom 26.06.1991.

Stadt Leverkusen (Hrsg.): Richtlinien der Stadt Leverkusen über Beihilfen zur Sportförderung, Beschluß des Rates vom 14.12.1992, ergänzt am 29.11.1993.

Stadt Limburg (Hrsg.): Richtlinien zur Förderung der sporttreibenden Vereine der Kreisstadt Limburg a.d.Lahn, Beschluß der Stadtverordnetenvertretung vom 17.12.1990.

Stadt Lörrach (Hrsg.): Richtlinien für die Förderung der Bereiche Soziales, Kultur, Jugend, Sport, Sonstiges vom 19.09.1985, geänderte Fassung vom 30.12.1992.

Stadt Lüdenscheid (Hrsg.): Richtlinien der Stadt Lüdenscheid zur Förderung des Sports in den Lüdenscheider Turn- und Sportvereinen in der Fassung vom 17.01.1989.

Stadt Mannheim (Hrsg.): Sportförderungsrichtlinien der Stadt Mannheim vom 17.12.1985, ergänzt im Jahr 1994.

Stadt Mönchengladbach (Hrsg.): Haushaltspläne 1975 bis 1993.

Stadt Neumünster (Hrsg.): Grundsätze der Stadt Neumünster über die Gewährung von finanziellen Beihilfen zur Förderung des Vereinssports (Sportförderungsgrundsätze), Neufassung gemäß Beschluß der Ratsversammlung vom 08.02.1994.

Stadt Neunkirchen (Hrsg.): Richtlinien für die Förderung vereinseigener Sportstätten bei Neubau- und Instandsetzungsarbeiten, Beschluß des Stadtrates vom 30.06.1993.

Stadt Neustadt a.Rbge. (Hrsg.): Richtlinien für die Sportförderung in der Stadt Neustadt a.Rbge., Beschluß des Rates vom 05.03.1992.

Stadt Neu-Ulm (Hrsg.) (1984): Sportförderungsrichtlinien, Auszug aus der Niederschrift über die öffentliche Sitzung des Hauptausschusses vom 22.11.1984.

----- (1989): Bau von Sportanlagen durch Vereine, Auszug aus der Sitzungsniederschrift des Hauptausschusses vom 20.09.1989.

Stadt Nürnberg (Hrsg.): Sport in der Stadt - Organisationen, Vereine, Förderungsrichtlinien, Neufassung gemäß Stadtratsbeschluß vom 08.04.1987. 
Stadt Offenburg (Hrsg.): Richtlinien für die Sport und Freizeitsportförderung vom 18.06.1991.

Stadt Osnabrück (Hrsg.): Richtlinien der Stadt Osnabrück über die Förderung des Sports (Sportförderungsrichtlinien) vom 28.03.1981 in der Fassung vom 27.12.1988.

Stadt Passau (Hrsg.): Richtlinien für die Vergabe der Sportförderungsmittel der Stadt Passau, Neufassung vom 14.05.1979, geändert gemäß Beschluß des Schul- und Sportausschusses vom 11.09.1990.

Stadt Pforzheim (Hrsg.) (1992): Sportförderung, Pforzheim August 1992.

---- (1993): Rundschreiben an die Pforzheimer Sportvereine zwecks vorübergehender Außerkraftsetzung der Sportförderungsrichtlinien aufgrund der finanziellen Situation der Stadt Pforzheim vom 25.05.1993.

Stadt Pinneberg (Hrsg.): Sportförderungsrichtlinie für die Stadt Pinneberg vom 01.01.1992.

Stadt Rastatt (Hrsg.): Richtlinien über die Gewährung von Zuschüssen an Rastatter Sportvereine, überarbeitete Fassung gemäß Gemeinderatsbeschluß vom November 1992.

Stadt Rendsburg (Hrsg.): Sportförderungsgrundsätze der Stadt Rendsburg, 1994.

Stadt Reutlingen (Hrsg.): Sportförderungsprogramm vom 21.11.1978, ergänzte Fassung vom 01.01.1988.

Stadt Sankt Augustin (Hrsg.): Richtlinien für die Sportförderung in der Stadt Sankt Augustin vom 04.12.1974 in der Fassung vom 16.12.1986.

Stadt Singen (Hrsg.): Sportförderungsrichtlinien, Beschlüsse des Gemeinderats der Stadt Singen (Hohentwiel) vom 13.10.1970, 28.03.1974, 14.03.1978, 31.10.1978, 16.01.1979, 25.11.1980, 16.09.1986 und 29.11.1988.

Stadt Solingen (Hrsg.): Richtlinien zur Sportförderung der Stadt Solingen, Neufassung gemäß Beschluß der Sitzung des Sportausschusses vom 15.08.1989.

Stadt Tübingen (Hrsg.): Sportförderungs-Richtlinien, überarbeitete, noch nicht beschlossene Fassung.

Stadt Tuttlingen (Hrsg.): Richtlinien für die Verteilung des städtischen Barzuschusses an die dem Stadtverband für Leibesübungen Tuttlingen angeschlossenen Sportvereine, Neufassung gemäß Beschluß der Mitgliederversammlung des Stadtverbands für Leibesübungen vom 17.10.1980. 
Stadt Ulm (Hrsg.) (1990): Richtlinien der Stadt Ulm für die Gewährung von Zuschüssen vom 15.03.1990.

----- (1993): Rundschreiben Nr. 6, Sportförderung, vom 15.03.1993.

Stadt Villingen-Schwenningen (Hrsg.): Sport in unserer Stadt, vom 18.09.1985.

Stadt Wetter (Hrsg.): Sportförderungsrichtlinien, Neufassung gemäß Änderungsbeschluß des Rates vom 20.09.1990.

Stadt Wetzlar (Hrsg.): Sportförderungsrichtlinien der Stadt Wetzlar, dritte Fassung vom 15.06.1992.

Stadt Wolfsburg (Hrsg.): Richtlinien für die Förderung des Sports in der Fassung vom 19.03.1986.

Stadt Worms (Hrsg.): Sportförderung in Worms, 1994.

Stadt Würselen (Hrsg.): Richtlinien der Stadt Würselen über die Gewährung von Zuwendungen zur Förderung des Sports, Beschluß des Rates der Stadt am 18.12.1990.

Stadt Würzburg (Hrsg.): Richtlinien für die Sportförderung der Stadt Würzburg, geänderte Fassung zum 01.01.1995.

Stamler, I. u.a.: Epidemiologic Studies of Cardiovascular Renal Diseases, in: Journal of Chronicle Diseases, 1960, S. 440 - 445.

Statistisches Bundesamt Wiesbaden (Hrsg.): Fachserie L, Finanzen und Steuern, Reihe 5, Sonderbeiträge zur Finanzstatistik, Aufwendungen von Bund, Ländern und Gemeinden (GV) für Gesundheitspflege und Sport, 1964 bis 1969.

---.- : Finanzen und Steuern, Reihe 1, Haushaltswirtschaft von Bund, Ländern und Gemeinden, II: Jahresabschlüsse, Kommunalfinanzen, 1965 bis 1969.

-.--- : Fachserie L, Finanzen und Steuern, Reihe 5, Sonderbeiträge zur Finanzstatistik, Ausgaben der öffentlichen Haushalte für Gesundheit, Sport und Erholung, 1970 bis 1973.

---- : Fachserie 14, Finanzen und Steuern, Reihe 3.6, Rechnungsergebnisse der öffentlichen Haushalte für Gesundheit, Sport und Erholung, 1974 bis 1979.

-.--. : Fachserie 14, Finanzen und Steuern, Reihe 3.5, Rechnungsergebnisse der öffentlichen Haushalte für soziale Sicherung und für Gesundheit, Sport, Erholung, 1980 bis 1991.

----- : Statistische Jahrbücher, 1960 bis 1993. 
Stiftung Verbraucher Institut (Hrsg.): Sportkonsum als Konsumsport?, Berlin 1987.

Stiglitz, J. E. u. Schönfelder, B: Finanzwissenschaft, 2. Auflage, München, Wien 1989.

Taylor, H. L. u.a.: Death rates among physically active and sedentary employers of Railroad - Industry, in: American Journal of Physical Health, 10/1962, S. 1697 1707.

Tettinger, P. J. (Hrsg.): Subventionierung des Sports, Heidelberg 1987.

Teufel, E. (Hrsg.): Sportförderung in Baden-Württemberg, Schriftenreihe der CDUFraktion im Landtag von Baden-Württemberg, Band II, Stuttgart 1980.

Thoma, M.: Theorie der öffentlichen Güter, in: WiSt, 1985/3, S. 125 - 130.

Tiebout, Ch. M.: A Pure Theory of Local Expenditures, in: Journal of Political Economy, 64/1956/10, S. $416-424$.

Timm, W.: Sportvereine in der Bundesrepublik Deutschland, Teil II: Organisations-, Angebots- und Finanzstruktur, Schriftenreihe des Bundesinstituts für Sportwissenschaft, Band 23, Schorndorf 1979.

Ulmer, H.-V.: Über das Thema Kosteneinsparung im Gesundheitswesen durch den Sport, in: Deutsche Zeitschrift für Sportmedizin, 40/1989/8, S. 291 - 292.

Vaz, E. E.: What price victory?, in: International Review of Sport Sociology, 1974/3-4, S. $33-55$.

Viererbl, O. J. (1975): Gemeindliche Sportausgaben 1974, in: Statistisches Jahrbuch deutscher Gemeinden, 62/1975, S. 216 - 239.

-.-- (1978): Gemeindliche Sportausgaben 1978, in Statistisches Jahrbuch deutscher Gemeinden, 66/1979, S. 251 - 275.

Wagner, A.: Grundlegung der politischen Ökonomie, 3. Auflage, erster Teil, zweiter Halbband, Leipzig 1893.

Weber, W.: Die wirtschaftliche Bedeutung des Sports, Schriftenreihe des Bundesinstituts für Sportwissenschaft, Band 81, Schorndorf 1995.

Weitzel, O.: Die Entwicklung der Staatsausgaben in Deutschland. Eine Analyse der öffentlichen Aktivität in ihrer Abhängigkeit vom wirtschaftlichen Wachstum, Diss., Erlangen, Nürnberg 1967.

Wicksell, K.: Finanztheoretische Untersuchungen - nebst Darstellung und Kritik des Steuerwesens Schwedens, Neudruck der Ausgabe Jena 1896, Aalen 1969. 
Winkler, J. u.a.: Verbände im Sport, Schriftenreihe des Bundesinstituts für Sportwissenschaft, Band 43, Schorndorf 1985.

Wössner, V.: Über Sportverletzungen, Diss., Tübingen 1966.

Zimmermann, H. u. Henke, K.-D.: Einführung in die Finanzwissenschaft, 5. Auflage, München 1987.

Zylmann, E.: Gesundheit und Sport, Marburg 1976.

\section{Gesetze:}

GemO Gemeindeordnung für Baden-Württemberg

GEMHVO Gemeindehaushaltsverordnung

Gesetz zur Förderung des Sports im Lande Berlin

Gesetz zur Förderung des Sports im Lande Bremen

Grundgesetz

Landesgesetz über die öffentliche Förderung von Sport und Spiel in Rheinland-Pfalz

\section{Zeitungen:}

Badische Zeitung

Die ZEIT

Frankfurter Allgemeine Zeitung

Mittelbayerische Zeitung

Süddeutsche Zeitung

WirtschaftsWoche 
Christian Hockenjos - 978-3-631-75159-6

Downloaded from PubFactory at 01/11/2019 07:36:54AM

via free access 


\section{FINANZWISSENSCHAFTLICHE SCHRIFTEN}

Band 1 Werner Steden: Finanzpolitik und Einkommensverteilung. Ein Wachstums- und Konjunkturmodell der Bundesrepublik Deutschland. 1979.

Band 2 Rainer Hagemann: Kommunale Finanzplanung im föderativen Staat. 1976.

Band 3 Klaus Scherer: Maßstäbe zur Beurteilung von konjunkturellen Wirkungen des öffentlichen Haushalts. 1977.

Band 4 Brita Steinbach: "Formula Flexibility" - Kritische Analyse und Vergleich mit diskretionärer Konjunkturpolitik. 1977.

Band 5 Hans-Georg Petersen: Personelle Einkommensbesteuerung und Inflation. Eine theoretisch-empirische Analyse der Lohn- und veranlagten Einkommensteuer in der Bundesrepublik Deutschland. 1977.

Band 6 Friedemann Tetsch: Raumwirkungen des Finanzsystems der Bundesrepublik Deutschland. Eine Untersuchung der Auswirkungen der Finanzreform von 1969 auf die Einnahmenposition der untergeordneten Gebietskörperschaften und ihrer regionalpolitischen Zieladäquanz. 1978.

Band 7 Wilhelm Pfähler: Normative Theorie der fiskalischen Besteuerung. Ein methodologischer und theoretischer Beitrag zur integration der normativen Besteuerungstheorie in der Wohlfahrtstheorie. 1978.

Band 8 Wolfgang Wiegard: Optimale Schattenpreise und Produktionsprogramme fŭr öffentliche Unternehmen. Second-Best Modelle im finanzwirtschaftlichen Staatsbereich. 1978.

Band 9 Hans P. Fischer: Die Finanzierung des Umweltschutzes im Rahmen einer rationalen Umweltpolitik. 1978.

Band 10 Rainer Paulenz: Der Einsatz finanzpolitischer Instrumente in der Forschungs- und Entwicklungspolitik. 1978.

Band 11 Hans-Joachim Hauser: Verteilungswirkungen der Staatsverschuldung. Eine kreislauftheoretische Inzidenzbetrachtung. 1979.

Band 12 Gunnar Schwarting: Kommunale Investitionen. Theoretische und empirische Untersuchungen der Bestimmungsgründe kommunaler Investitionstätigkeit in NordrheinWestfalen 1965-1972. 1979.

Band 13 Hans-Joachim Conrad: Stadt-Umland-Wanderung und Finanzwirtschaft der Kernstădte. Amerikanische Erfahrungen, grundsătzliche Zusammenhänge und eine Fallstudie für das Ballungsgebiet Frankfurt am Main. 1980.

Band 14 Cay Folkers: Vermögensverteilung und staatliche Aktivität. Zur Theorie distributiver Prozesse im Interventionsstaat. 1981.

Band 15 Helmut Fischer: US-amerikanische Exportförderung durch die DISC-Gesetzgebung. 1981.

Band 16 Günter Ott: Einkommensumverteilungen in der gesetzlichen Krankenversicherung. Eine quantitative Analyse. 1981.

Band 17 Johann Hermann von Oehsen: Optimale Besteuerung. (Optimal Taxation). 1982.

Band 18 Richard Kössler: Sozialversicherungsprinzip und Staatszuschūsse in der gesetzlichen Rentenversicherung. 1982.

Band 19 Hinrich Steffen: Zum Handlungs- und Entscheidungsspielraum der kommunalen Investitionspolitik in der Bundesrepublik Deutschland. 1983.

Band 20 Manfred Scheuer: Wirkungen einer Auslandsverschuldung des Staates bei flexiblen Wechselkursen. 1983. 
Band 21 Christian Schiller: Staatsausgaben und crowding-out-Effekte. Zur Effizienz einer Finanzpolitik keynesianischer Provenienz. 1983.

Band 22 Hannelore Weck: Schattenwirtschaft: Eine Möglichkeit zur Einschränkung der öffentlichen Verwaltung? Eine ökonomische Analyse. 1983.

Band 23 Wolfgang Schmitt: Steuern als Mittel der Einkommenspolitik. Eine Ergänzung der Stabilitătspolitik? 1984.

Band 24 Wolfgang Laux: Erhöhung staatswirtschaftlicher Effizienz durch budgetäre Selbstbeschränkung? Zur Idee einer verfassungsmăßig verankerten Ausgabengrenze. 1984.

Band 25 Brita Steinbach-van der Veen: Steuerinzidenz. Methodologische Grundlagen und empirisch-statistische Probleme von Länderstudien. 1985.

Band 26 Albert Peters: Ökonomische Kriterien für eine Aufgabenverteilung in der Marktwirtschaft. Eine deskriptive und normative Betrachtung für den Allokationsbereich. 1985.

Band 27 Achim Zeidler: Möglichkeiten zur Fortsetzung der Gemeindefinanzreform. Eine theoretische und empirische Analyse. 1985.

Band 28 Peter Bartsch: Zur Theorie der längerfristigen Wirkungen 'expansiver' Fiskalpolitik. Eine dynamische Analyse unter besonderer Berücksichtigung der staatlichen Budgetbeschränkung und ausgewählter Möglichkeiten der öffentlichen Defizittinanzierung. 1986.

Band 29 Konrad Beiwinkel: Wehrgerechtigkeit als finanzpolitisches Verteilungsproblem. Möglichkeiten einer Kompensation von Wehrungerechtigkeit durch monetäre Transfers. 1986.

Band 30 Wolfgang Kitterer: Effizienz- und Verteilungswirkungen des Steuersystems. 1986.

Band 31 Heinz Dieter Hessler: Theorie und Politik der Personalsteuern. Eine Kritik ihrer Einkommens- und Vermögensbegriffe mit Blick auf die Leistungsfăhigkeitstheorie. 1994.

Band 32 Wolfgang Scherf: Die beschäftigungspolitische und fiskalische Problematik der Arbeitgeberbeiträge zur Rentenversicherung. Eine Auseinandersetzung mit der Kritik an der lohnbezogenen Beitragsbemessung. 1987.

Band 33 Andreas Mästle: Die Steuerunion. Probleme der Harmonisierung spezifischer Gütersteuern. 1987.

Band 34 Günter Ott: Internationale Verteilungswirkungen im Finanzausgleich der Europäischen Gemeinschaften. 1987.

Band 35 Heinz Haller: Zur Frage der zweckmäßigen Gestalt gemeindlicher Steuern. Ein Diskussionsbeitrag zur Gemeindesteuerreform. 1987.

Band 36 Thomas Kuhn: Schlüsselzuweisungen und fiskalische Ungleichheit. Eine theoretische Analyse der Verteilung von Schlüsselzuweisungen an Kommunen. 1988.

Band 37 Walter Hahn: Steuerpolitische Willensbildungsprozesse in der Europäischen Gemeinschaft. Das Beispiel der Umsatzssteuer-Harmonisierung. 1988.

Band 38 Ulrike Hardt: Kommunale Finanzkraft. Die Problematik einer objektiven Bestimmung kommunaler Einnahmemöglichkeiten in der gemeindlichen Haushaltsplanung und im kommunalen Finanzausgleich. 1988.

Band 39 Jochen Michaelis: Optimale Finanzpolitik im Modell überlappender Generationen. 1989.

Band 40 Bernd Raffelhüschen: Anreizwirkungen der sozialen Alterssicherung. Eine dynamische Simulationsanalyse. 1989.

Band 41 Berend Diekmann: Die Anleihe- und Darlehenstransaktionen der Europäischen Gemeinschaften. 1990.

Band 42 Helmut Kaiser: Konsumnachfrage, Arbeitsangebot und optimale Haushaltsbesteuerung. Theoretische Ergebnisse und mikroökonometrische Simulation für die Bundesrepublik Deutschland. 1990. 
Band 43 Rüdiger von Kleist: Das Gramm-Rudman-Hollings-Gesetz. Ein gescheiterter Versuch der Haushaltskonsolidierung. 1991.

Band 44 Rolf Hagedorn: Steuerhinterziehung und Finanzpolitik. Ein theoretischer Beitrag unter besonderer Berücksichtigung der Hinterziehung von Zinserträgen. 1991.

Band 45 Cornelia S. Behrens: Intertemporale Verteilungswirkungen in der gesetzlichen Krankenversicherung der Bundesrepublik Deutschland. 1991.

Band 46 Peter Saile: Ein ökonomischer Ansatz der Theorie der intermediären Finanzgewalten Die Kirchen als Parafisci. 1992.

Band 47 Peter Gottfried: Die verdeckten Effizienzwirkungen der Umsatzsteuer. Eine empirische allgemeine Gleichgewichtsanalyse. 1992.

Band 48 Andreas Burger: Umweltorientierte Beschäftigungsprogramme. Eine Effizienzanalyse am Beispiel des "Sondervermögens Arbeit und Umwelt". 1992.

Band 49 Jeanette Malchow: Die Zuordnung verteilungspolitischer Kompetenzen in der Europäischen Gemeinschaft. Eine Untersuchung aufgrund einer Fortentwicklung der ökonomischen Theorie des Föderalismus. 1992.

Band 50 Barbara Seidel: Die Einbindung der Bundesrepublik Deutschland in die Europäischen Gemeinschaften als Problem des Finanzausgleichs. 1992.

Band 51 Ralph Wiechers: Markt und Macht im Rundfunk. Zur Stellung der öffentlich-rechtlichen Rundfunkanstalten im dualen Rundfunksystem der Bundesrepublik Deutschland. 1992.

Band 52 Klaus Eckhardt: Probleme einer Umweltpolitik mit Abgaben. 1993.

Band 53 Oliver Schwarzkopf: Die Problematik unterschiedlicher Körperschaftsteuersysteme innerhalb der EG. 1993.

Band 54 Thorsten Giersch: Bergson-Wohlfahrtsfunktion und normative Ökonomie. 1993.

Band 55 Li-Fang Chou: Selbstbeteiligung bei Arneimitteln aus ordnungspolitischer Sicht. Das Beispiel der Bundesrepublik Deutschland. 1993.

Band 56 Harald Schlee: Einkommensteuertiche Behandlung von Transferzahlungen. Zur Neuordnung der Familienbesteuerung sowie der Besteuerung von Versicherungsleistungen und Sozialtransfers. 1994.

Band 57 Alexander Spermann: Kommunales Krisenmanagement. Reaktionen baden-württembergischer Stadtkreise auf steigende Sozialhilfekosten und Einnahmenausfälle (198092). 1993.

Band 58 Otto Roloff / Sibylle Brander / Ingo Barens / Claudia Wesselbaum: Direktinvestitionen und internationale Steuerkonkurrenz. 1994.

Band 59 Claudia Wesselbaum-Neugebauer: Intemationale Steuerbelastungsvergleiche. 1994.

Band 60 Stephanie Miera: Kommunales Finanzsystem und Bevölkerungsentwicklung. Eine Analyse des kommunalen Finanzsystems vor dem Hintergrund der sich abzeichnenden Bevölkerungsentwicklung am Beispiel Niedersachsens unter besonderer Berücksichtigung des Landkreises Wolfenbüttel und seiner Gemeinden. 1994.

Band 61 Wolfgang Schert: Die Bedeutung des kaldorianischen Verteilungsmechanismus für die gesamtwirtschaftlichen Wirkungen der staatlichen Neuverschuldung. 1994.

Band 62 Rainer Volk: Vergleich der Vergünstigungseffekte der verschiedenen investitionsfördernden Maßnahmen. 1994.

Band 63 Hans-Georg Napp: Kommunale Finanzautonomie und ihre Bedeutung für eine effiziente lokale Finanzwirtschaft. 1994. 2., unveränderte Auflage 1994.

Band 64 Bernd Rahmann / Uwe Steinborn / Günter Vornholz: Empirische Analyse der Autonomie lokaler Finanzwirtschaften in der Europäischen Gemeinschaft. 1994. 
Band 65 Carsten Kühl: Strategien zur Finanzierung der Altlastensanierung. 1994.

Band 66 Stephan Boll: Intergenerationale Umverteilungswirkungen der Fiskalpolitik in der Bundesrepublik Deutschland. Ein Ansatz mit Hilfe des Generational Accounting. 1994.

Band 67 Karl Justus Bernhard Neumärker: Finanzverfassung und Staatsgewalt in der Demokratie. Ein Beitrag zur konstitutionellen Finanztheorie. 1995.

Band 68 Christian Haslbeck: Zentrale versus dezentrale Internalisierung externer Effekte bei unvollständiger Information. 1995.

Band 69 Regina Müller: Horizontale oder vertikale Transfers zur Durchsetzung eines horizontalen Finanzausgleichs. 1995.

Band 70 Christian Hockenjos: Öffentliche Sporttörderung in der Bundesrepublik Deutschland. Darstellung und finanztheoretische Analyse. 1995. 